\title{
An Integrated Knowledge Management Model for Community Enterprises: \\ A Case Study of a Rural Community Enterprise in Thailand
}

\author{
by \\ Lanthom Jonjoubsong BSc, MSc \\ A thesis submitted to Victoria University of Wellington \\ in fulfillment of the requirements for the degree of \\ Doctor of Philosophy
}

School of Information Management

Faculty of Commerce and Administration

Victoria University of Wellington

2008 


\begin{abstract}
This research seeks to explore the characteristics, organisational culture and current knowledge management (KM) practice in a community enterprise (CE). Prae Pun-a hand weaving $\mathrm{CE}$ in the northeast rural area of Thailand-has typical CE characteristics — self-employed and collaborative management, and a home-based production system utilising local resources and knowledge. This research used a qualitative method. Data were collected through narrative inquiry, participant observation and document analysis. The 18 respondents were Prae Pun members, committee members, an advisory committee member and office staff members. The data were analysed through theme analysis.
\end{abstract}

The findings of this research reveal that, although community members would not be aware of doing so, Prae Pun managed its knowledge through informal educational activities-learning by doing and observation-and work processes concerned with collaboration and informality. The current KM practice of Prae Pun entails three processes: basic skill development, competence building and new knowledge creation through creation, capture, sharing, transferring, verification, utilisation and codification. Significantly for this study, KM of Prae Pun focuses on organisational knowledge creation and indigenous knowledge systems. The current KM of Prae Pun also has four other components. Its knowledge resources come from members, other villagers, other hand weaving groups, support organisations and markets. Most of the knowledge is tacit and indigenous. The operation and management systems determine the knowledge processes and provide supportive and incentive systems. The organisational culture and resources, including funding, social capital and appropriate technologies, also provide supportive and incentive systems. The resources are also the tools that facilitate the knowledge processes. The knowledge assets encompass actual assets-products, cloth designs, techniques, and operation and management systems - and competencies including members' skills and abilities, social capital, and shared values, which are not registered as intellectual properties but are in the forms of organisational memories. 
The traditional KM practice of Prae Pun can be possibly strengthened by integrating modern KM concepts, including knowledge resources from academic and government agencies, appropriate technologies to facilitate knowledge codification and knowledge transfer, extrinsic reward and incentive systems, and facilitators or consultants to enhance members' communities of practice (CoPs). The findings of this study and the KM model contribute to both CE development and further research on KM in a CE context and indigenous KM. 


\section{Acknowledgements}

I would like to thank all those who helped to make this thesis possible. First, I would like to express my gratitude to my principal supervisor, Professor Gary Gorman, for his excellent direction throughout this research. He provided both guidance and challenge in the scholarly process. His thoughtful scholarship steered me on the right track to complete my thesis. I also would like to thank Dr Brian Harmer, my cosupervisor, who provided support for my work and gave me much to think about in wider contexts. Many thanks go to staff members of the Student Learning Support Service at Victoria University of Wellington (VUW), especially Dr Xiaodan Gao and Dr Deborah Laurs, who provided excellent support and assistance during my studies. I also would like to thank the VUW PhD Submission Scholarship for financial support in the last stage of my PhD study.

Second, I am grateful to Prae Pun members who were willing to participate in the narrative enquiry needed to complete the data collection for this thesis. I would like to especially thank Ms Darunee Supawan-an advisory committee member of Prae Pun — who introduced me to Prae Pun and provided much support for my work. My thanks also go to the lovely villagers in the seven villages of Prae Pun and the children at Nongthum village whose joyfulness, cheerfulness and kindness helped me to feel at home with the villagers of Prae Pun.

Third, I would like to take this opportunity to thank Huachiew Chalernprakiet University - my employing organisation—for financially supporting my personal and professional development through higher degree study.

Fouth, thanks also go to my friends and colleagues for their encouragement and support during my study. Of special note are my best friends-Korsak Nopraratruangden, Werapong Prapha, Udorn Wanarrom and Piyakhun Nopprakhunfor encouragement and supporting me.

Finally, I would like to express my deepest gratitude to my parents, my sister and my brothers and their families for their support and love. I would like to dedicate all 
success of this thesis to my parents-Mrs. Songwan and Mr. Prathum Jonjoubsongwho always encourage, support and love me. 


\begin{tabular}{|c|c|}
\hline BP & British Petroleum \\
\hline CAQDAS & Computer assisted qualitative data analysis software \\
\hline CEs & Community enterprises \\
\hline $\mathrm{CoP}$ & Communities of practice \\
\hline GDP & Gross Domestic Product \\
\hline FTO & Fair trade organisation \\
\hline \multirow[t]{2}{*}{ ICLS } & The Fifteenth International Conference of Labour \\
\hline & Statisticians \\
\hline ICT & Information and communication technology \\
\hline IK & Indigenous knowledge \\
\hline ILO & International Labour Organisation \\
\hline IS & Information system \\
\hline IT & Information technology \\
\hline KM & Knowledge management \\
\hline KMI & Knowledge management institute Thailand \\
\hline MEs & Micro enterprises \\
\hline MSEs & Micro and small enterprises \\
\hline NGO & Non-governmental organisation \\
\hline \multirow[t]{2}{*}{ NUDIST } & Non-numerical Unstructured Data Indexing Searching and \\
\hline & Theorising \\
\hline NWD & The handicraft centre for Northeast Women's Development \\
\hline SMEs & Small and medium enterprises \\
\hline \multirow[t]{2}{*}{ STAR } & The Strengthening, Training, and Rural Development \\
\hline & Project \\
\hline TID & Trade and Investment Division \\
\hline TRN & Thai Rural Net \\
\hline UNDP & United Nations Development Programme \\
\hline
\end{tabular}




\section{Table of contents}

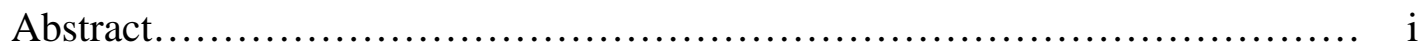

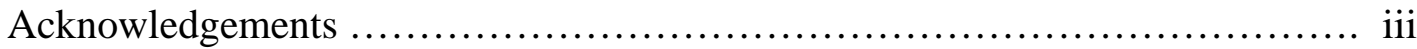

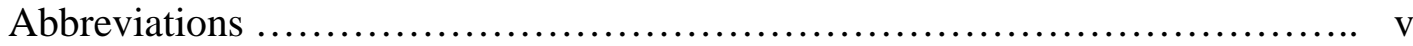

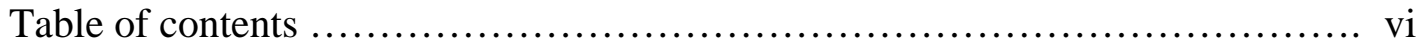

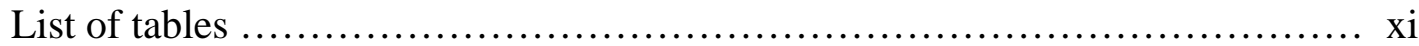

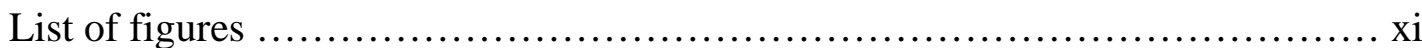

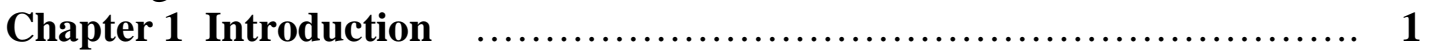

1.1 Important and motivation ........................................ 1

1.2 Statement of problems ............................................. 2

1.3 Research objectives and research questions ......................... 3

1.4 Theoretical framework ............................................. 3

1.5 Research design, limitations and delimitations of study ................. 4

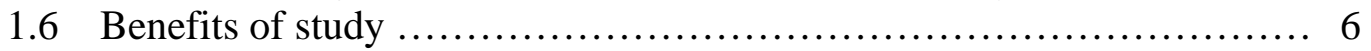

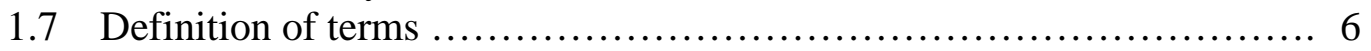

1.8 Organisation of the thesis. ........................................... 8

Chapter 2 Literature Review ................................................ 10

2.1 Micro and community enterprises .................................... 10

2.1.1 Definition of micro and community enterprises ................. 10

2.1.2 Characteristics of micro and community enterprises ............... 12

2.1.2.1 Owners and location ................................... 12

2.1.2.2 Types of business ...................................... 12

2.1.2.3 Operational aspects .................................... 13

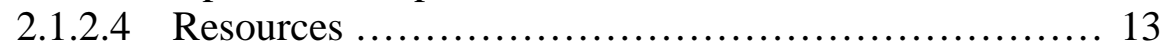

2.1.3 Roles of micro and community enterprises ..................... 16

2.1.3.1 Economic development ............................... 16

2.1.3.2 Natural resource conservation ........................ 17

2.1.3.3 Gender equality ................................... 17

2.1.3.4 Civil institution development ........................ 17

2.1.4 Micro and community enterprises in Thailand ................... 18

2.1.4.1 Characteristics of micro and community enterprises in Thailand ........................................... 18

2.1.4.2 Roles of micro and community enterprises in Thailand 19

2.2 Organisational knowledge ......................................... 21

2.2.1 Knowledge and organisational knowledge ...................... 21

2.2.2 Knowledge types .............................................. 23

2.2.2.1 Declarative knowledge ................................. 23

2.2.2.2 Procedural knowledge .................................... 23

2.2.2.3 Theoretical knowledge .................................. 24

2.2.2.4 Personal knowledge ................................... 24

2.2.3 Particular knowledge elements .................................. 25

2.2.3.1 Indigenous knowledge .............................. 25

2.2.3.2 Experience ........................................... 28

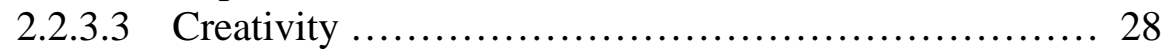

2.2.3.4 Collective senses and shared values ...................... 29

2.3 Knowledge management ............................................... 29

2.3.1 Knowledge management approaches ........................... 29

2.3.2 Knowledge management for micro enterprises ..................... 31

2.3.3 Knowledge processes ............................................ 32 
2.3.3.1 Knowledge creation ................................ 33

2.3.3.2 Knowledge capture and codification .................... 35

2.3.3.3 Knowledge sharing ............................... 36

2.3.3.4 Knowledge transfer ............................... 37

2.3.3.5 Knowledge verification ............................. 39

2.3.3.6 Knowledge utilisation ............................. 40

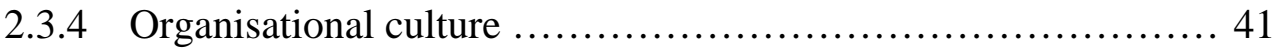

2.3.4.1 National culture .................................. 41

2.3.4.2 Organisational culture ............................ 46

2.3.4.3 Knowledge management-driven culture ............... 49

2.3.5 Organisational management ............................... 51

2.3.5.1 Organisational learning ............................. 51

2.3.5.2 Collaborative environment .......................... 53

2.3.5.3 Reward and incentive systems .................... 54

2.3.6 Appropriate technologies for KM ............................. 55

2.4 Knowledge management in Thailand .............................. 58

2.4.1 Knowledge management models for Thai society ............... 58

2.4.2 Basic knowledge management for KM in Thailand ............. 68

2.4.2.1 BP's KM model .................................... 68

2.4.2.2 Xerox's KM model ................................. 70

2.5 Knowledge management for community enterprises .................. 72

2.6 The theoretical framework ......................................... 73

Chapter 3 Research methodology ....................................... 77

3.1 Research paradigm ............................................... 77

3.2 Methodology approaches .......................................... 77

3.3 Narrative research ............................................. 79

3.3.1 Definition and characteristics of narrative research ............. 79

3.3.2 Narrative research in organisation and KM research ............ 80

3.3.3 Narrative research for this research ........................ 81

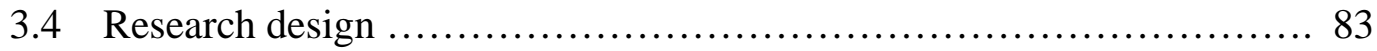

3.4.1 Research site ............................................. 84

3.4.2 Data collection ............................................. 85

3.4.2.1 Document analysis ................................. 86

3.4.2.2 Narrative inquiry ............................... 87

3.4.2.3 Participant observation ............................ 88

3.4.3 Data analysis ........................................... 89

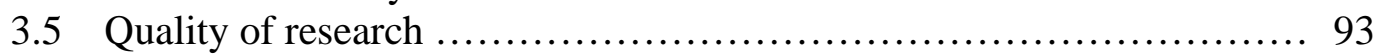

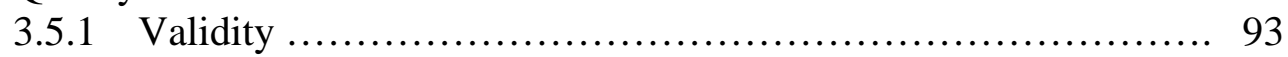

3.5.2 Credibility ................................................ 94

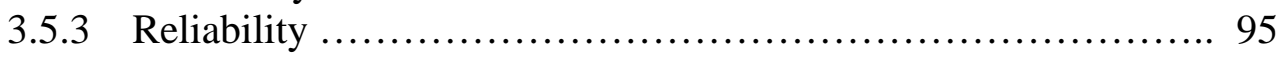

3.5.4 Ethical issues ........................................... 95

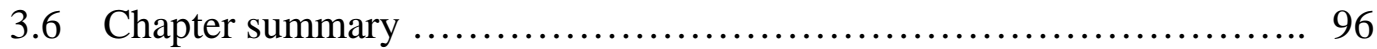

Chapter 4 Research procedures ....................................... 97

4.1 Research site selection and access .................................. 97

4.2 Access to respondents ........................................ 98

4.3 Roles of the researcher ........................................... 99

4.4 Data gathering ..................................................... 100

4.4.1 Document study ............................................. 100

4.4.2 Narrative inquiry ............................................ 102

4.4.3 Participant observation ....................................... 104 
4.5 Data analysis ................................................... 104

4.5.1 Transcribing ................................................. 105

4.5.2 Identifying themes ........................................... 105

4.5.3 Coding for themes .......................................... 106

4.5.4 Data presentation ....................................... 106

$\begin{array}{lll}4.6 & \text { Chapter summary } & 106\end{array}$

Chapter 5 Prae Pun's Stories ........................................ 107

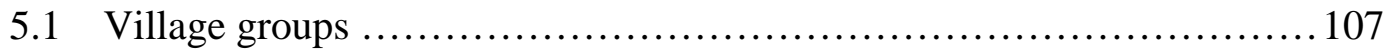

5.1 .1 Being members ............................................. 108

5.1.2 Cloth and weaving ............................................114

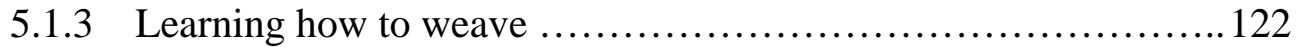

5.1 .4 Natural dyes ................................................ 127

5.2 Being committee members ........................................ 132

5.3 Office work ....................................................... 137

5.3.1 At the office .................................................. 137

5.3.2 At handicraft fairs ............................................ 141

5.4 Advisory committee members ................................... 142

5.5 Chapter summary .................................................. 144

Chapter 6 Characteristics of Prae Pun ............................... 146

6.1 Introduction to Isan region and culture ............................ 146

6.2 Background and establishment ................................. 148

6.3 Objectives of Prae Pun .......................................... 151

6.4 Operational and management systems ............................... 152

6.4.1 Committee systems ........................................ 153

6.4.1.1 Board committee .................................. 153

6.4.1.2 Village groups ..................................... 155

6.4.1.3 Members ............................................ 156

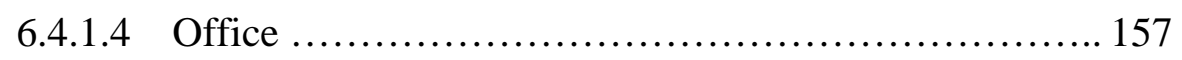

6.4.2 Open discussion ............................................ 158

6.4.3 Self-employment system .....................................159

6.4.4 Job sharing ............................................... 160

6.4.5 Production systems ........................................ 160

6.4.6 Marketing management ..................................... 161

6.5 Organisational culture .............................................. 162

6.5.1 Community spirit .......................................... 163

6.5.1.1 Influence of Isan culture ..............................163

6.5.1.2 Influence of Thai culture ............................... 165

6.5.1.3 Influence of Buddhist beliefs .......................... 165

6.5.2 The sense of ownership .................................... 165

6.5.3 Informal orientation ........................................ 167

6.5.4 Participation orientation ................................... 168

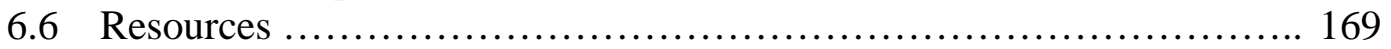

6.6.1 Prae Pun's knowledge ......................................170

6.6.1.1 Knowledge of hand weaving .......................... 170

6.6.1.2 Knowledge of natural dyes ......................... 171

6.6.1.3 Knowledge of community enterprise management ..... 172

6.6.2 Financial resource ........................................ 173

6.6.3 Information and communication technologies .................. 173

6.6.4 Social capital ........................................... 174

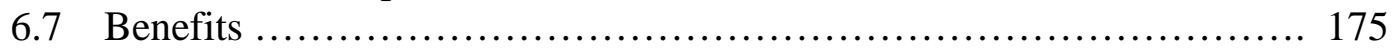


6.7.1 Income .................................................... 175

6.7.2 Business ownership and dividends ........................... 176

6.7.3 Welfare to members and their families ......................... 176

6.7.4 Intangible benefit ......................................... 177

6.8 Chapter summary .................................................. 177

Chapter 7 Prae Pun's knowledge processes ............................ 179

7.1 Prae Pun's knowledge processes ................................... 179

7.2 Basic skill creation ................................................ 179

7.2.1 Generic skill creation ..................................... 180

7.2.1.1 Generic skills at hand weaving ..................... 180

7.2.1.2 Generic skills at natural dyes ...................... 182

7.2.2 Contextual skill creation ..................................... 183

7.2.2.1 Orientation and trial period ........................... 184

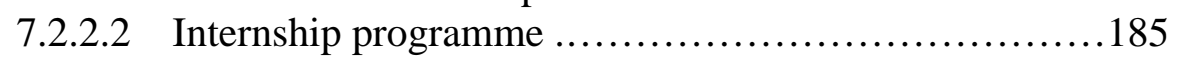

7.2.2.3 Committee support programme ...................... 186

7.3 Competence building ........................................... 187

7.3.1 Further knowledge capture .............................. 187

7.3.1.1 Study tours and sharing forums ..................... 188

7.3.1.2 Training activities................................ 190

7.3.1.3 Working ....................................... 192

7.3.1.4 Document study ................................ 196

7.3.2 Capture of particular techniques .............................. 198

7.3.2.1 Experimentation ..................................... 198

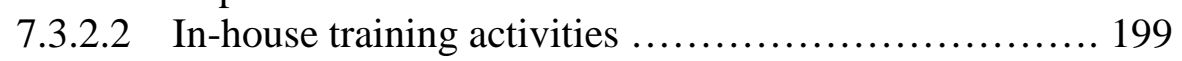

7.3.2.3 Local expert involvement ......................... 200

7.3.3 Further skill development ................................. 200

7.3.3.1 Production skill development ....................... 200

7.3.3.2 Business operation and management skill development 201

7.4 New knowledge creation ....................................... 203

7.4.1 Experimentation ............................................ 204

7.4.1.1 Individual experimentation ......................... 204

7.4.1.2 Group experimentation .............................. 205

7.4.2 Working ................................................... 206

7.4.3 Serendipitous discovery ................................... 207

7.5 Conclusion for knowledge processes of Prae Pun ..................... 208

7.5.1 Knowledge processes ..................................... 208

7.5.1.1 Knowledge creation ................................ 208

7.5.1.2 Knowledge capture and sharing ..................... 209

7.5.1.3 Knowledge transfer .............................. 210

7.5.1.4 Knowledge verification ............................ 210

7.5.1.5 Knowledge codification ............................. 210

7.5.1.6 Knowledge utilisation ............................ 211

7.5.2 Organisational knowledge creation ........................... 211

7.5.3 Indigenous knowledge systems ............................ 213

7.6 Chapter summary ................................................ 214

Chapter 8 A Knowledge Management Model for Prae Pun ................. 216

8.1 Knowledge management model for Prae Pun ........................ 216

8.1.1 Knowledge ................................................. 218

8.1.1.1 Knowledge from members ....................... 219

8.1.1.2 Knowledge from villagers .......................... 222 
8.1.1.3 Knowledge from other hand weaving groups .......... 222

8.1.1.4 Knowledge from support organisations ................. 223

8.1.1.5 Knowledge from markets ........................... 223

8.1.2 Operation and management systems ......................... 224

8.1.2.1 Determining of knowledge processes ................. 224

8.1.2.2 Supportive and incentive systems .................. 225

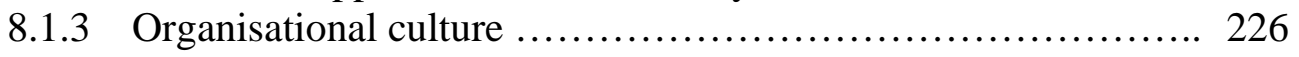

8.1.3.1 Knowledge management-driven culture ............. 227

8.1.3.2 Reward and incentive systems ..................... 228

8.1.4 Resources ................................................ 228

8.1.4.1 Funding ....................................... 229

8.1.4.2 Social capital ........................................ 229

8.1.4.3 Appropriate technologies ............................. 230

8.1.5 Knowledge processes ..................................... 231

8.1.5.1 Basic knowledge capture ........................... 232

8.1.5.2 Further knowledge capture ........................ 232

8.1.5.3 New knowledge creation .......................... 234

8.1.6 Organisational knowledge assets ............................. 236

8.1.6.1 Assets ......................................... 236

8.1.6.2 Competences .................................... 237

8.2 Strengthening the KM model ...................................... 238

8.2 .1 Knowledge resources ..................................... 238

8.2.2 Operation and management systems ......................... 239

8.2.3 Resources .................................................... 240

8.2.4 Knowledge processes ...................................... 241

8.2.5 Knowledge assets .......................................... 243

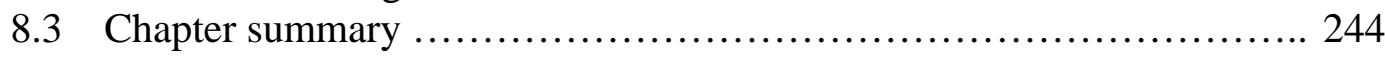

Chapter 9 Conclusions and implications ............................... 245

9.1 Conclusions of the research ..................................... 245

9.1.1 Objectives and research questions ......................... 245

9.1.2 Research design .......................................... 246

9.1.3 The findings of this research ................................ 246

9.1.3.1 Answer to research question one ..................... 246

9.1.3.2 Answer to research question two .................... 247

9.2 Implications of the research ...................................... 248

9.2.1 Implications for Practice .................................. 248

9.2.1.1 Implications for Prae Pun ........................... 248

9.2.1.2 Implications for other CEs .......................... 249

9.2.2 Limitations of this research and implications for further research 250

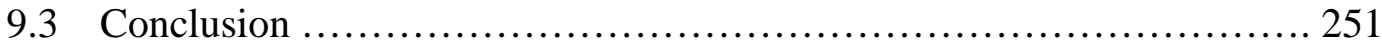

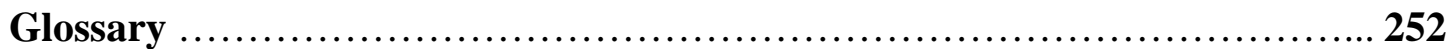

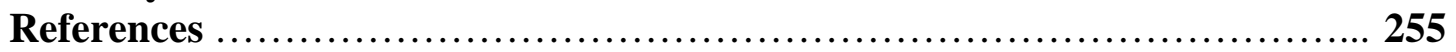

Appendices ............................................................ 272

Appendix A: Human Ethic Approval Documents ........................ 272

Appendix B: List of respondents .................................... 285

Appendix C: An example of transcripts of the narratives in English .......... 286

Appendix D: Data analyses documents .............................. 294

Appendix E: Basic knowledge of hand weaving and natural dyes .......... 309 


\section{List of tables}

Table 2.1 National culture dimension scores of Geert Hofstede for Thailand in 1991 (Hofstede, 1991)........................... 43

Table 8.1 The characteristics of Prae Pun .................................... 216

Table 8.2 The current knowledge management practice of Prae Pun .............21

Table E1 An examples of data coding of the narratives of Mrs. Raweewan in the category of organisational knowledge ........................ 296

Table E2 An examples of data coding of the narratives of Mrs. Raweewan in the category of knowledge processes

\section{List of figures}

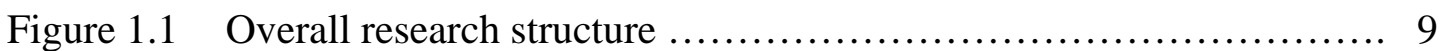

Figure 2.1 The randomly recursive knowledge processes ..................... 33

Figure 2.2 The first KM model of KMI Thailand adapted from KMI data(2005a,

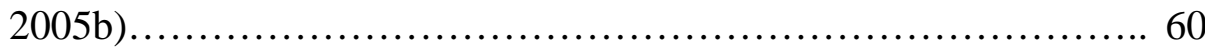

Figure 2.3 Self-assessment table used to evaluate core competencies of groups or

group members (KMI, 2005b)................................ 62

Figure 2.4 A river diagram (KMI, 2005b) ................................ 63

Figure 2.5 A stair diagram drawn from levels of current core competency and target core competency (KMI, 2005b) ....................... 64

Figure 2.6 Carp model, adapted from KMI data (2005a, 2005b) ............... 65

Figure 2.7 Carp model with "Thran Pannya” (River wisdom) as a knowledge sharing Tool adapted from KMI data (2005a, 2005b)................ 67

Figure 2.8 Carp model with Xerox model as a knowledge sharing tool adapted from KMI data (2005a, 2005b) ................................ 68

Figure 2.9 BP's Knowledge Management model, adapted from Collison and Parcell (2004) ................................................ 69

Figure 2.10 The Eureka model: knowledge sharing model for service staff of Xerox adapted from Holtshouse (1999), Kankanhalli et al. (2003) and Roberts- Witt (2005) ...........................................

Figure 2.11 A theoretical framework for studying KM model for community Enterprises ................................................ 76

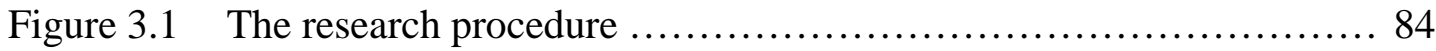

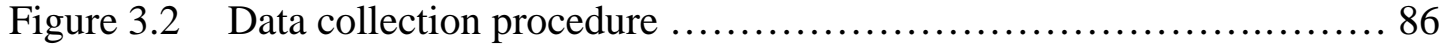

Figure 3.3 Data analysis framework of this research ....................... 91

Figure 3.4 Qualitative data analysis as an iterative procedure (Dey, 1993) ...... 91

Figure 3.5 Data analysis model of Miles and Huberman (1994) ................... 92

Figure 4.1 Procedures of narrative data analysis ............................ 105

Figure 5.1 Khon Kaen map with indicators for Prae Pun village groups (Picture from http://www.khonkaencricket.com/images/kkarea1b\%20 shrunk.jpg) ................................................. 108

Figure 5.2 Khon Kaen village life style in summer: woman hand weaving on ground floor beside relaxing area ............................ 109

Figure 5.3 '4-kao' loom with a narrow reed and four foot pedals for rug making ................................................. 109

Figure 5.4 A weaving hut of two members at Nongthum village ............... 120 
Figure 5.5 A committee meeting environment ........................... 133

Figure 5.6 Prae Pun office and showroom: from the external side .............. 138

Figure 5.7 Internal Prae Pun office and showroom ........................... 138

Figure 6.1 Household layout and current Isan home style ...................... 146

Figure 6.2 The ground floor of Isan household has a traditional bench

for relaxing, eating and socializing, and a weaving loom ............147

Figure 6.3 Khon Kaen map with indicators showing locations of

Prae Pun village groups ........................................149

Figure 6.4 Geography of the areas where Prae Pun villages located ............. 152

Figure 6.5 The organisational structure of Prae Pun ............................ 153

Figure 7.1 The knowledge processes of Prae Pun ............................. 179

Figure 7.2 All knowledge processes of Prae Pun ............................. 208

Figure 7.3 Matching of the knowledge processes of the proposed KM model

with the organisational knowledge creation model of Nonaka (1994) 212

Figure 8.1 A current knowledge management model for Prae Pun .............. 218

Figure 8.2 Knowledge resources for the KM model of Prae Pun ................ 219

Figure 8.3 The roles of operation and management systems for the

KM model of Prae Pun ........................................ 224

Figure 8.4 Organisational cultures as supportive and incentive systems for the KM model of Prae Pun .......................................... 227

Figure 8.5 Resources for the KM model of Prae Pun ......................... 229

Figure 8.6 The knowledge processes of the KM model of Prae Pun .............. 232

Figure D1 A classification for data analysis .................................... 294

Figure D2 Codes for data classification of knowledge processes ............... 295

Figure E1 A traditional Isan hand weaving loom ............................ 309

Figure E2 A weaving loom with thong leang heddle shaft as a supplement part (noyshop.com, 2004) ....................................... 311

Figure E3 A diamond float pattern of a Prae Pun big blanket ................... 311

Figure E4 An example kit design ...................................... 312

Figure E5 An example mudmee design ................................... 313

Figure E6 An example of Bun Phawes banner .............................. 313 


\section{Chapter 1}

\section{Introduction}

\subsection{Importance and motivation}

Community enterprises (CEs) are group-owned enterprises which are founded by community members living in the same areas. The main objective of CEs is community development through the enhancement of the quality of community life (Welsch and Kuhns, 2005). CEs play an important role in the generation of income and jobs for community members, gender equality, civil institution development and the conservation of natural resources. Micro and community enterprises can create job opportunities and income for poor people, especially for those in rural areas and for women in particular (Halvorson-Quevedo, 1991; Kitahara, 2000; Wijayaratna, 2000). In terms of gender equality, micro and community enterprises allow women to demonstrate their abilities in business operations. Women have skills in agricultural production, food processing and handicrafts (United Nations, 1995; ILO, 2000b) which are the main businesses of most micro and community enterprises. Micro and community enterprises allow women to undertake both housework and paid work because most of these enterprises are a part of the informal sector which is based mainly in the home (Halvorson-Quevedo, 1992; ILO, 2000a; WIEGO, 2005). In terms of civil institution development, CEs play an important role in strengthening grassroots communities by increasing human capital, social capital (Welsch and Kuhns, 2005), and ability in problem solving and resource management (Halvorson-Quevedo, 1992). Finally, CEs can increase the awareness of community members about natural resources conservation. Most micro and community enterprises are agriculture-based or handicraft producers which rely on natural and local resources (Tambunan, 2000). CEs in Thailand play an important role in community development just as CEs in other developing countries do, and have the same characteristics.

However, CEs have many obstacles restricting their growth. Members run their businesses with limited business skills and few resources including human, financial and technological resources. CEs fall into the micro enterprise category and the informal sector. Most members of CEs are self-employed with low levels of education and limited management skills (TID, 2003). In terms of financial resources, CEs have 
minimal capital from their owners. As part of the informal sector, they are nonregistered enterprises, and thus they have few opportunities for formal support and loans. These businesses are based on local resources, human capital (i.e. employees' skills and experiences), and social capital rather than investment in technology (Halvorson-Quevedo, 1991). These characteristics can lead to CEs lacking skills in business development including production and service, marketing, and information and knowledge management systems.

Even though micro and community enterprises are production-based (Shama, Miller and Reeder (1990) and labour-intensive (Nelson, 1987), they run businesses based on traditional skills and local resources, using artisanal techniques and local wisdom, rather than advanced technologies (Nelson, 1987; Halvorson-Quevedo, 1991). In a sense, they run businesses which rely on human capital and indigenous knowledge. Therefore, increasing their knowledge management potential would help CEs to fully exploit their resources and strengthen their business and community development.

Thus, this research into knowledge management (KM) for CEs will focus on local knowledge with traditional practice. In order to enhance the traditional practice of CEs with modern KM, a KM model will be specifically designed by carefully investigating their operational systems, organisational culture and current knowledge management practice. This is necessary because KM has not been studied in a CE context. This study will focus on the investigation of a hand-weaving community enterprise in the north-eastern rural area of Thailand (Prae Pun) as a representative CE.

\subsection{Statement of problems}

There have been many KM models proposed for large enterprises and small and medium enterprises (SMEs) which focus on social, organisational, and technological aspects, but cannot be applied directly to CEs. This is because the characteristics of CEs are different from those of larger enterprises. Community enterprises are not only small in size, but they also have specific objectives of community development which involve different operational systems and organisational culture. They are group owned enterprises which have group management and networking systems (Waliasatian, 1996). In a sense, members manage the enterprises as collaborative 
systems in order to share ideas and experience, and help each other with selfdevelopment. The members of CEs are community members who live in the same areas. Their relationships are participation oriented (Halvoson-Quevedo, 1991) and informal (ILO, 2000a). Due to the community orientation and limits of finance and technology, the characteristics of CEs mean that their communication focuses on faceto-face communication rather than technological orientation. Their businesses rely more on human capital and social capital rather than financial and technological resources. These characterisitics can be summarised into three issues as follows:

1) Community enterprises have specific operational and management systems, and an organisational culture that is different from large, and small and medium enterprises.

2) They rely on human capital and social capital rather than finance and advanced technologies.

3) They have limited resources including human, financial and technological resources.

\subsection{Research objectives and research questions}

This research seeks to explore the characteristics, organisational culture, and KM capabilities of a representative CE, and to develop a possible KM model for CEs. The KM model will address community enterprises’ characteristics, organisational culture, constraints, in terms of social, operational and technological aspects. The research will aim to answer two research questions:

1. How does the community enterprise currently manage its knowledge?

2. Can theoretical and modern KM concepts be applied in an appropriate manner to strengthen traditional KM practice of the community enterprise?

\subsection{Theoretical framework}

Knowledge can be defined as 'justified true belief' emerging from experiencing, reflection and inference processes (Nonaka, 2002; Audi, 2003). Knowledge is derived from human minds through understanding and justification and related through human action processes (Jones, 1964; Davenport and Prusak, 1998; Nonaka, 2002). In the organisational context, knowledge is a valuable asset that is available to support 
organisational activities. It involves organisational members and actions (Pan and Scarbrough, 1999). KM in organisations focuses on the utilisation of knowledge to create value for organisations by creating, acquiring, storing and deploying knowledge for products and services (Grant, 1996). Knowledge management is defined as a conceptual framework of activities and perspectives in order to gain, deal with, and benefit from the corporation's knowledge assets (Wiig, 1994). KM is concerned with many disciplines including business and organisational management, sociology, philosophy, psychology, communication, and information management and information and communication technology (ICT) (Skyrme, 1999; Wiig, 2000; Gamble and Blackwell, 2001; Prusak, 2001). This study will consider KM in terms of human capital management systems or process orientation, rather than through artefact orientation or an IT-based approach. The system will focus on four main components - enterprise operational and management, organisational culture, and resources, including human capital, technology and social capital-as factors to determine the knowledge processes and characteristics of the Prae Pun KM model in order to create an organisational knowledge asset.

\subsection{Research design, limitations and delimitations of the study}

\subsubsection{Research design}

This research uses qualitative methods with narrative research. This is because narrative research focuses on the lives and experience of individuals in connection with social events and other people (Clandinin and Connelly, 2000; Creswell, 2005). Significantly, according to the nature of the narrative research discussed by Elliott (2005), narrative research allows the researcher to deeply explore the CE through the meaning of participants' narratives, social contexts and the temporality of the accounts.

Data are collected through narrative inquiry and ethnographic techniques_-participant observation and document analysis. This is because qualitative data and rich data require naturalistic inquiry which takes place in a natural setting with open-end inquiry (Silverman, 2005; Williamson, 2006). Prae Pun—a rural CE in Thailand-has been selected as a research site because it has characteristics which are also typical of other CEs. Then, the data are analysed with narrative analysis which focuses on investigation and interpretation of the data for the provision of answers for research 
questions or research texts (Riessman, 1993; Clandinin and Connelly, 2000; Creswell, 2005).

\subsubsection{Limitations of the study}

As the research site of this study is a hand-weaving community enterprise in northeast rural Thailand, there are some limitations and risks to be considered.

\subsubsection{Willingness of respondents}

Given the research site, most respondents are rural women who are farmers and perform weaving as a supplementary job at home. Generally, they were willing to give information in convenient places, such as their homes or villages; in their local language (Isan dialect); and to familiar people. Thus, data collection took place at their homes or communal places, such as village halls and temples, after the researcher was introduced to them.. The researcher stayed in the community with enterprise members' families in order to become familiar with the respondents and to participate in activities with the respondents. In addition, the researcher prepared herself to be familiar with the Isan dialect by using the Isan dialect with other members and villagers before collecting data.

\subsubsection{Availability of respondents}

As mentioned above, most of the respondents are farmers. The best time for data collection would be after harvesting and before planting, i.e. between February and May, If some research activities could not be conducted in those periods, suitable times would be in the evening and at their villages.

\subsubsection{Delimitation of study}

There are some limitations and risks that might affect the proposed research, e.g. the number of cases and respondents. Given the limited time available for this study, it focuses on a single case study. In order to collect a comprehensive range of opinions, the respondents for the in-depth study comprise at least one member from each village, and at least one person in each position in the enterprise. 


\subsection{Benefits of the study}

Community enterprises have different characteristics and operating systems from those of large organisations and SMEs. Since CEs have limited human, financial, and technological resources, they need to be examined in order to improve understanding of their components and to identify an appropriate KM model. This study provides two kinds of benefits: academic value and social development.

\subsubsection{Contribution to the literature}

Although there have been a number of knowledge management studies which are concerned with organisational culture and ICT, they have tended to focus on large businesses, especially consulting firms and cross-cultural enterprises. That is why this research contributes an understanding of traditional KM for a CE and an analysis of how modern KM can add value to the traditional practice. The researcher investigates the complex interaction of organisational culture, operational systems and KM practice of a CE through the case study of a rural community enterprise in Thailand. Thus, this research creates a suitable KM model, integrating organisational culture, current situations and current KM practice with the modern concepts of KM, which could strengthen the traditional practice of CEs.

\subsubsection{Contribution to practitioners}

This research is partly aimed at developing the community enterprise and rural communities. It enhances awareness of the use of KM to develop their enterprise, realising their capabilities and the advantages of KM. Thus, the research offers a KM model which community enterprises could use as a resource for development projects in order to enhance their current KM practice.

\subsection{Definition of terms}

Appropriate technologies Information and communication technologies that are suitable for micro and community enterprises

Community enterprise Community enterprises are micro enterprises which can be either formal or informal, are community owned and have members who belong to communities in the same regions. 
Human capital

Human resource

Indigenous knowledge

Informal sector

Knowledge

KM-driven culture

Local wisdom

Micro and small enterprise
Human capital includes employees' knowledge, skills, experience, and wisdom (Lin, 2001).

Human resources are the employees who carry human capital.

Indigenous knowledge refers to traditional and local knowledge possessed by groups of people living in a particular area for a long period of time (Rao, 2006; Langill, 2007).

The informal sector includes organisations involved in a wide range of activities including casual work, unofficial, business activities, and underground business activities (World Bank Group, 2005). This study focuses on production units that have a low level of organisation, small scale, casual employment, and unincorporated enterprises (ILO, 2000a).

Knowledge is recognition which arises from understanding (Jones, 1964) and is also regarded as the understanding and abilities of people and organisations gained from experiences (Wiig, 2000; Leitch and Rosen, 2001) which Lin (2001) considers human capital.

An organisational culture influenced by KM. This study focuses on sharing and learning cultures.

Local wisdom is knowledge integrated with indigenous knowledge, ethical principles and culture, and adapted to be appropriate to local societies, environment and culture (Thai Knowledge Centre, 2007). It is indigenous knowledge from the perspective of eastern thought (Small, 2004).

Micro and small enterprise (MSE) is a category that groups micro enterprises and small enterprises together (White, 1999). 
Micro enterprise

Small enterprise

Usable knowledge
An enterprise that has less than five employees including the self-employed (White, 1999).

An enterprise that has 5-19 employees (White, 1999).

Procedural knowledge which is verified for its appropriateness for particular situations, and concerned with practical understanding and abilities to enable organisational members to perform activities.

\subsection{Organisation of the thesis}

This research report can be viewed in three parts. Part one (chapter 2) covers the literature review, theoretical framework and research questions. Part two (chapters 3 and 4) outlines the methodology and research design. Part three (chapters 5-9) presents the research findings from the fieldwork in Prae Pun in order to answer the first research question, and introduces a model of the CE's current KM practice, in order to address the second research question. Figure 1.1 shows the overall structure of the thesis. 


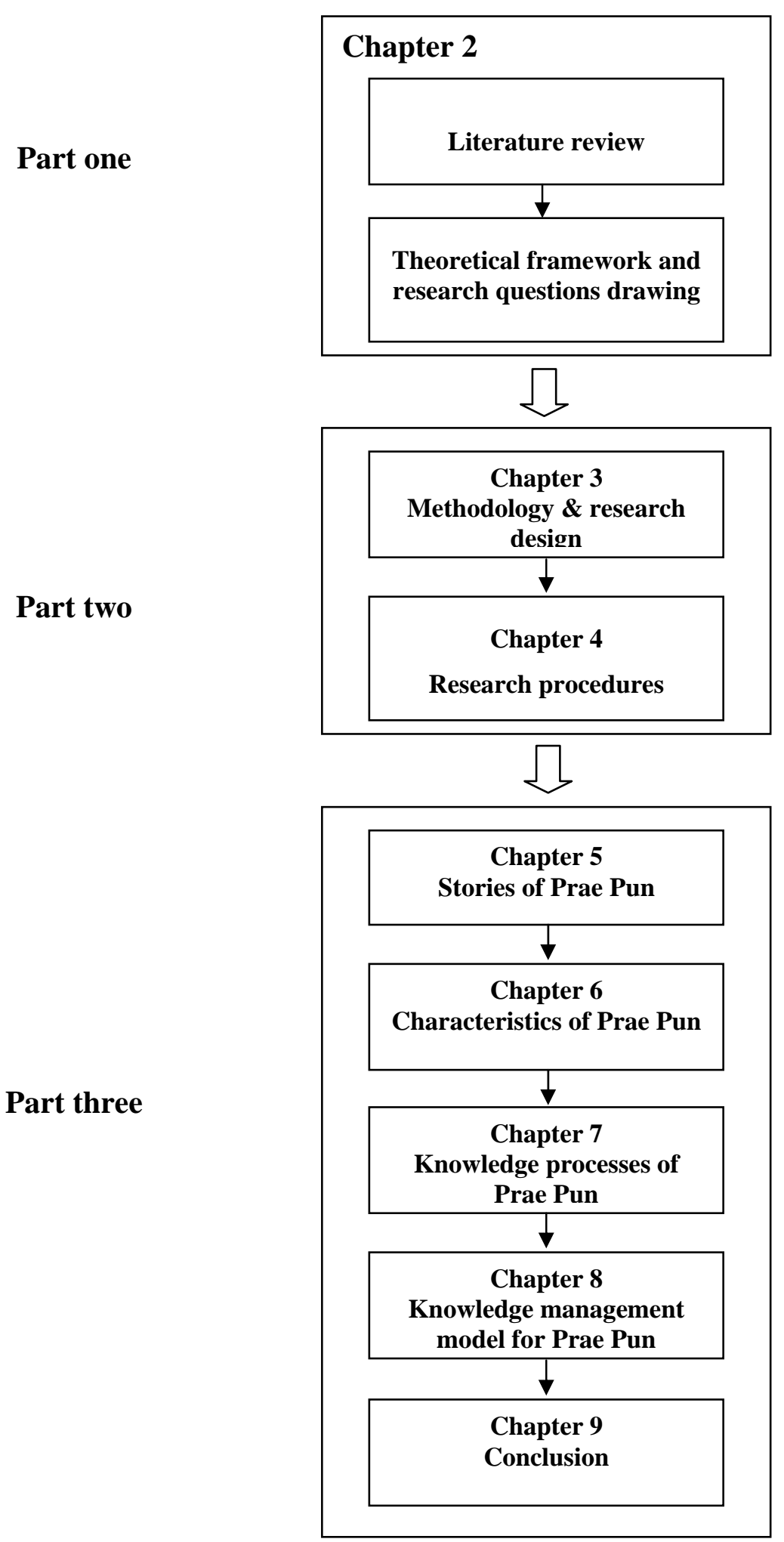

Figure 1.1 Overall thesis structure 


\section{Chapter 2}

\section{Literature Review}

Four main disciplines are reviewed for this study. The discussion of the literature begins with the characteristics and roles of micro and community enterprises, including the situation in Thailand. The second part is concerned with organisational knowledge. Part three discusses the knowledge management components that are relevant to community enterprises. The final part examines both knowledge management for community enterprises and knowledge management in Thailand.

\subsection{Micro and community enterprises}

To understand community enterprises (CEs), it is necessary to define them. Then, we can focus on these enterprises' characteristics and their roles. The last part of this section describes the characteristics and roles of community enterprises in Thailand.

\subsubsection{Definition of micro and community enterprises}

There are many types of business. Generally, they are classified by size based on the number of employees and amount of capital investment, and operational styles. In 1993, the International Labour Organisation (ILO) distinguished four enterprise sizes: cottage, small, medium-sized, and large. In 1999, the ILO and the United Nations Development Programme (UNDP) renamed cottage enterprises as micro enterprises (MEs). These have fewer than five employees, including the self-employed. Small enterprises have 5-19 employees, medium-sized enterprises have 20-49 employees, and the large ones have 50 or more employees (White, 1999). In Thailand, in 1998, the government defined small enterprises as having fewer than 50 employees and registered capital of no more than ten million Baht ${ }^{1}$; medium enterprises as those with 50-200 employees and registered capital of 10-100 million Baht; and SMEs as those with less than 200 employees and less than 100 million Baht of registered capital (White, 1999). In practice, the categories are combined into micro and small enterprises (MSEs), small and medium enterprises (SMEs), and large enterprises. This is not only because of the size of the enterprises but also their business operation systems. Wasuntiwongse (1999) defines MSEs as enterprises that are small and

\footnotetext{
${ }^{1}$ \$NZ 1 is around 25 Baht
} 
conduct all their business functions by themselves, while medium-size and large enterprises manage their businesses using skilled managers.

Community enterprises (CEs) can be defined as MSEs because it is generally agreed that CEs operate their businesses on a small scale with self-employed members and a small amount of capital. In fact, CEs can be classified as a specific type of enterprise. They are different from general MSEs in that the latter can be privately owned and self-managed, but CEs are community-owned and operated by a group and have a larger number of members or self-employed workers. Wasuntiwongse (1999) found that a CE in Thailand usually has around 30-50 members. Furthermore, CEs have different objectives from MSEs. CEs are established with the basic objectives of community development through enhancing the quality of community life and economic development (Welsch and Kuhns, 2005). According to the community enterprise development centre in Thailand (2001), the main purposes of CEs are to enhance economic and social development, and provide community learning opportunities. Waliasatian (1996) defines CEs as group or community-owned organisations. Their business operation is related to the community's economic and social situation, and lifestyles. They do basic production and provide services using local and natural materials, simple technology, local wisdom, and a local labour force which is low in business skills.

Therefore, CEs in this research mean the specific type of MSEs which have small scale and informal operation systems, but are community-owned and operated, and whose main purpose is community development. However, the literature review includes the terms MEs, CEs and community business studies. This is because, at the World Bank and NGO Forum in 1999 at Birmingham University, the participants defined enterprises that are founded by communities as MEs, CEs, or community businesses (Petprasert and Wongkul, 2002). Therefore, any reports and information about MEs published after the forum may also refer to community enterprises or community businesses. In order to facilitate clear understanding of CEs, this section will cover both MEs and CEs. 


\subsubsection{Characteristics of micro and community enterprises}

Four main characteristics of MEs and CEs are considered: owners and location, types of business, operational aspects, and resources.

\subsubsection{Owners and location}

MEs and CEs aim to enhance community development in order to reduce poverty. Most MEs and CEs are established by poor people and social development agencies in developing countries. Welsch and Kuhns (2005) indicate that CEs are concerned with grassroots and sustainable organisations because they are founded by local people and focused on local business. They can be found in many countries in Asia, Africa, America and Europe, such as India, Thailand, Tanzania, Poland, the US, and Mexico (Datta, 2000; Mwaisela, 2000). In Africa, most MEs are located in rural areas while in Latin America they are mostly urban (Halvorson-Quevedo, 1991). In Asia, most MEs are located in rural areas (Amin, 2002). Tambunan (2000) also found that most MSEs in Thailand and Indonesia are rural. This is because the vast majority of poor people live in the countryside. CEs may also be mainly located there because rural people are more community-oriented. Nevertheless, urban areas also have communities where members have migrated from rural areas (Burke, 1970). Thus, they would still be regarded as community-oriented. Wasantiwongse (1999) finds that MEs in Thailand also do their business on a group basis and most business activities take place at community gathering places, such as community halls and temples.

\subsubsection{Types of business}

Generally, MEs specialise in labour-intensive work (Nelson, 1987; HalvorsonQuevedo, 1991) and simple manufacturing and service businesses (Shama, Miller and Reeder, 1990). They set up businesses related to their skills and local resources and use artisanal techniques and local wisdom rather than investing in advanced technology (Nelson, 1987; Halvorson-Quevedo, 1991). Wijayaratna (2000) indicates that rural community enterprises in India have included handicrafts, handlooms, sericulture, and coir. In Indonesia, Tambunan (2000) found that over half the MSEs are involved in agriculture-based industry and handicraft production, using agricultural materials as their main raw materials. It can be observed that most MEs in rural areas are production-oriented enterprises, based on owners' skills and local resources. 


\subsubsection{Operational aspects}

Regarding the classification and ownership of MEs and CEs, these are minimal in structure and use casual operation systems. This is because CEs are community-based as discussed in the previous section. Furthermore, they are influenced by NGO characteristics, as NGOs are the main support organisations for MEs (see next section). Hudson (1995) finds that NGOs have an informal working environment. They focus on participation and decision-making (Bernard, 1984; Paton and Cornforth, 1992) and are equally accountable to many stakeholders (Hudson, 1995). These characteristics can influence the MEs' focus on informal operations. Most MEs and CEs are unregistered enterprises which are part of the informal sector (HalvorsonQuevedo, 1991). They do business through casual employment and relationships rather than formal contracts (ILO, 2000a). However, this classification only applies to MEs in developing countries. This is because MEs in these countries have simple business operations without regulations and taxes, whereas in developed countries the same enterprises are more complex and subject to regulation and tax systems (Schreiner, 2001). Wasuntiwongse (1999) finds that MEs in Thailand have tax exemptions and few regulations. Nevertheless, being part of the informal sector can result in a lack of support and services from governments and formal agencies (Halvorson-Quevedo, 1991).

On the other hand, the informal operation systems of MEs can be an advantage for knowledge management. This is because they are participant-oriented and creative (Halvorson-Quevedo, 1991), which can influence knowledge sharing and creation in organisations.

\subsubsection{Resources}

Micro enterprises and CEs tend to use local and natural resources and have limited financial assets and support. The Trade and Investment Division (2003) states that workers in rural agriculture-based enterprises in Asia and the Pacific lack business skills because they have low levels of education and insufficient training, poor motivation and few incentives, and a lack of opportunities for system improvement. As mentioned previously, they rely on local resources and local wisdom. The local resources include raw materials, human capital and social capital. 


\section{Human capital}

Human capital is a part of knowledge asset or intellectual capital (Stewart, 1997). Human capital is intangible and embodied in skills and capabilities. Human capital is derived through experience and learning in both formal and informal education (Davison and Honig, 2003). Because staff members have low levels of education, MEs' human capital would be derived mainly from experience, practical learning and informal education through their occupancy and life. Thus, the human capital of MEs is associated with indigenous or traditional knowledge. Indigenous knowledge (IK) refers not only to the knowledge of aboriginal people (Storey, 2005), but to knowledge that is derived from long-term occupancy of people in particular areas through adaptation to local conditions (Sefa Dei, Hall and Rosenberg, 2000; Hansen and VanFleet, 2003). As a result, with this human capital, CEs have specific knowledge and skills to make their businesses become unique. Furthermore, even though MEs specialise in labour-intensive work (Nelson, 1987; Halvorson-Quevedo, 1991), they can be referred to knowledge-intensive enterprises that not only possess expertise from more highly-educated staff members, but also in the forms of experience, organisational routines and culture, which can be utilised for the benefit of the organisations (Starbuck, 1992).

\section{Social capital}

Because CEs are community-oriented, they tend to have strong relationships, trust, and shared values which combine to make high social capital. Social capital is a social asset or resource that can be obtained through social relations and connections (Lin, 2001). Social capital is collective (Bourdieu, 1986) and valuable capital, which can facilitate the actions of a particular community (Coleman, 1988). It can be seen in the forms of social networks, social norms, obligations and expectations (Coleman, 1988). Nahapiet and Ghoshal (1998) also consider that social capital can be viewed in three dimensions: structural, relational and cognitive. First, a social network is concerned with social relations and social structure which provide information channels for the network (Coleman, 1988; Hoffman, Hoelscher and Sherif, 2005). The social structure is concerned with the patterns of connections of community members which involves network ties (Nahapiet and Ghoshal, 1998). The social capital in this context can provide values and information to facilitate actors (Coleman, 1988) and be a channel for information transmission (Nahapiet and Ghoshal, 1998). 
Second, social capital in the form of social norms is focused on collective norms which involve foregoing self interest and acting in the interest of society (Coleman, 1988). The social capital in this context refers to social control in organisations (Hoffman, Hoelscher and Sherif, 2005) and a degree of consensus in the social system (Coleman, 1988). According to Lin (2001), social capital in this context refers to power. It can be seen that the social capital is a crucial factor for organisations to facilitate the action of members.

Finally, social capital in the forms of obligations and expectations comprises reputation which provides collective sanctions to ensure trustworthiness from members of the network and people outside of the network, according to Hoffman, Hoelscher and Sherif (2005). They state that individuals in a social structure that has a high level of obligations have more social capital in activities or high availabilities. The social capital in this context can be found in societies in which members have restricted relations (Bourdieu, 1986; Burt, 1992), such as extended families and traditional villages (Coleman, 1988). It is arguable that CEs which are group-based would have higher social capital and utilise it for their business operations. For example, Jommuang (1997) finds that CEs in Thailand work together as a network. Hantrakul (2000) also notes that successful community enterprises in Thailand have high levels of member participation and trust in their managerial teams.

Given this, CEs utilise social capital for their business development, especially to develop their competencies, because social capital in the form of social networks can provide information and channels for transmission from social connections and social relations. Meanwhile, social capital in other forms can encourage CEs' members in learning and intellectual creation. Coleman (1988), and Nahapiet and Ghoshal (1998) deem that social capital can facilitate human capital creation. Likewise, Capello and Faggian (2005) find that social capital can encourage the learning and innovation of organisations.

\section{Financial resources}

As mentioned previously, MEs and CEs are a part of the informal sector. Furthermore, as they are unregistered and have few assets, MEs may have difficulty getting loans from financial institutions. Halvorson-Quevedo (1991) found that MEs 
generally obtain financial support and technical assistance from non-governmental organisations (NGOs). In addition, MEs normally get financial support through group loans using social capital as credit (Conning, 1999). Thus, this characteristic can affect MEs' capacity for improvement including investment in technology, and development of production, business operations, and human resources.

In short, the characteristics of MEs and CEs are unique and different from SMEs and large enterprises. These characteristics lead to different organisational cultures and management styles. Furthermore, the characteristics can be influenced by knowledge management factors as will be discussed in section 2.3.

\subsubsection{Roles of micro and community enterprises}

As mentioned previously, MEs and CEs are established to solve social problems and develop communities in areas such as economic development, natural resources conservation, gender equality, and civil institution development.

\subsubsection{Economic development}

The main purposes of MEs and CEs are to generate income and increase local employment. MEs and CEs can create work and income for poor people and those with a low level of education, especially rural people, and women. Kitahara (2000) finds that over 35 per cent of the working population in rural Thailand work in the informal sector, especially women, and the middle-aged and elderly who are forced to retire from the formal sector. In Sri Lanka and India also, rural and community-based enterprises generate a large amount of income and job opportunities for rural communities (Wijayaratna, 2000). Halvorson-Quevedo (1991) indicates that 30-70 per cent of the working population in developing countries has worked in the informal sector including MSEs, and contributed 20-70 per cent of GDP. Furthermore, MEs were expected to create about 44 million jobs per year between 1991 and 2000. It can be inferred that MEs and CEs can be a solution in developing countries for economic problems, such as the economic crisis in Southeast Asia in mid 1997, when many workers from the formal sector returned to rural areas and switched to the informal sector, and MEs and CEs. 


\subsubsection{Natural resource conservation}

As mentioned previously, MEs are mostly agriculture-based and production-oriented enterprises, and tend to rely on local materials. Even though they use natural resources as raw materials for their production, they pay attention to natural resource conservation which can affect production and services. Prae Pun, a hand weaving enterprise in rural Thailand, has an interest in natural resources conservation in order to protect the trees that are the raw materials for its production (Hutanuwatr and Hutanuwatr, 2002). As a result, MEs can be crucial in increasing the awareness and participation of community members in natural resource conservation.

\subsubsection{Gender equality}

MEs and CEs can provide opportunities for women to show their abilities and potential in business and social development. For example, these enterprises allow women to demonstrate their abilities in operating businesses. Many MEs and CEs rely on women's skills, including handicraft production and food processing (United Nations, 1995; ILO, 2000b). In terms of social development, women can potentially perform the roles of both generating income and looking after their families through working in MEs and CEs. Generally, women are discriminated against in the formal sector, especially in developing countries (Halvorson-Quevedo, 1991; ILO, 2000b). In Vietnam, women have limited opportunities in the formal sector because they lack experience and knowledge, especially of foreign languages and legal issues (Truong, 2002). In Thailand, women, especially the middle-aged and elderly, are the first to be let go when businesses need to reduce staff (Kitahara, 2000). In the informal sector, women can undertake both housework and paid work, because MEs are mainly based in the home (Halvorson-Quevedo, 1991; WIEGO, 2005).

\subsubsection{Civil institution development}

CEs can be an effective tool for grass-roots institution development, because they provide not only economic solutions but also human capital and social capital (Welsch and Kuhns, 2005). First, CEs play an important role in creating human capital. CEs can markedly improve members' abilities in solving problems, managing resources and generating ideas through the enterprises' activities (HalvorsonQuevedo, 1991). According to Lin (2001), the skills and abilities that organisational members gain are considered to be human capital, which contributes to organisations' 
production and work processes. Second, as discussed previously, CEs are communityoriented which enhances the degree of social relations and frequency of networking in communities. Thus, CEs can increase the human capital and social capital of communities, which are crucial factors in enhancing the self-confidence and selfreliance of community members in order to strengthen civil society.

\subsubsection{Micro and community enterprises in Thailand}

2.1.4.1 Characteristics of micro and community enterprises in Thailand It is arguable that CEs in Thailand have the same characteristics as other CEs in developing countries in terms of owners, type of business, operational aspects and resources, and are located in rural areas, as are most CEs in Asia.

With regard to owners and types of business, CEs in Thailand were mainly established by cooperatives under the control of the Ministry of Agriculture and Cooperatives, and NGOs, but currently most CEs are independent and owned by communities (Petprasert, 1997). Most CEs in Thailand are in the informal sector and are larger than individual MEs. They have around 30-50 self-employed members involved in production. Indeed, they have managed the enterprises as micro businesses (Wasuntiwongse, 1999).

As for business types, most MEs in Thailand are located in rural areas and focus on handicrafts, furniture, and processing food and agricultural products (Romijn cited in Tambunan, 2000). Wasuntiwongse (1999) finds that MEs in Bangkok and Petchaburi province are mainly involved in food and garment production. Furthermore, CEs in hill tribe communities concentrate on food processing and agricultural production (Narintharangkul-na-Ayuthaya, 2002).

The operation systems of MEs and CEs in Thailand are casual and simple, like those of MEs and CEs in other countries. The CEs in Bangkok and Phetchaburi province conduct business in an informal way. They manage their businesses with committees and and business activities take place in communal areas, such as community halls and temples, with production processes based in members' homes (Wasuntiwongse, 1999). The community enterprises in the hill tribes are similar, organising their 
businesses as a group but with individual production systems (Narintharangkul-naAyuthaya, 2002).

Finally, MEs and CEs in Thailand use local and limited human, technological and financial resources, with a focus on social capital. As mentioned previously, the major business types of CEs in Thailand are handicrafts, food processing and agricultural production. They rely on local materials, and skills and knowledge from CE members and community members. Committee members are selected from within the enterprise, and the type of product chosen by each enterprise is based on the skills of each community. There are many examples of this, such as the success of the Ruk Thammachat Club Rice Mill—considered a CE-which relies on social capital including community participation, strong trust in leadership (Hantakul, 2000), and loyalty to the chairman. The Ban Pradoak Thai noodle group committee also plays an important role in that enterprise's operation (Pamapimai, 2001). In terms of local skills, the focus of this study, the Prae Pun hand weaving community enterprise in northeast Thailand, depends on the skills and knowledge of its members in natural dyeing and local hand weaving methods. In terms of finance, Prae Pun was established by members' investment and support from NGOs in the form of coordination and facilitators for business operations (Hutanuwatr and Hutanuwatr, 2002). CEs in Thailand are similar to CEs in other developing countries in terms of their lack of business skills, knowledge of technology, commercial laws and regulations, and marketing (Wasuntiwongse, 1999).

\subsubsection{Roles of micro and community enterprises in Thailand}

CEs in Thailand have been established with the underlying objectives of economic and social development. They play the same important roles as community enterprises all over the world, i.e. providing income generation and employment development, natural resource conservation, gender equality, and civil institution development.

In terms of economic development, CEs in Thailand play an important role as a source of employment and supplementary income for rural people. For example, the herbal shampoo group at Ban Thasai generates employment for the community by giving members the opportunity to grow herbs which they then supply to the business as raw materials (Pealnain, 1998). Romijn (cited in Tambunan, 2000) found that 
around 20 per cent of the value generated by manufacturing comes from rural enterprises. The Thai noodle group at Ban Pradoak is now the main source of income for the community (Pamapimai, 2001). It can help members to improve their families' quality of life. For example, the enterprise's members have used the income to pay for their children’s education.

As businesses that depend on local and natural materials, CEs in Thailand have to pay attention to natural resources conservation. Gamchoei (2001) claims that CEs' activities can create awareness about local resources protection, because community members recognise the relationship between local resources and their CE's activities.

In terms of improving gender equality, CEs provide opportunities for rural women to participate in social development. There are many CEs focussed on developing women's potential and abilities and relying on women's skills, such as local hand weaving and food processing.

As for civil institution development, CEs provide significant ways of enhancing the human capital and social capital of communities. CEs provide activities such as learning processes to build up the skills and abilities of their members. The Community Health Promotion Centre in northeast Thailand conducts traditional health forums and training programmes as the part of their business to educate their staff and members (Trinutchakorn 2002). CEs are group-based enterprises and, therefore, enhance social capital by running businesses with communities in the same areas or with the same interests. Thus, CEs can increase the levels of trust and relationships, which strengthens community development.

It is clear that, although CEs belong in the category of MEs and the informal sector, CEs are unique, with distinctive characteristics including the founders, purposes and roles of the enterprise, operation systems and resources. CEs in Thailand are similar to other CEs, especially those in developing countries in Asia, such as Indonesia, Vietnam, India, and Sri Lanka. As such, they play very important roles in social development. Therefore, the strength of CEs indicates the strength of communities at the grass-roots level. 


\subsection{Organisational Knowledge}

2.2.1 Knowledge and organisational knowledge

Knowledge can be considered as an abstract thing which can be seen in many forms and located everywhere around us. Knowledge resides in many locations and things, such as in human heads, skills, action (Newell, Robertson, Scarbrough and Swan, 2002), work practices (Brown and Duguid, 1998; Davenport and Prusak, 1998), organisation routines (Badaracco, 1991; Davenport and Prusak, 1998), procedure manuals, books and other codified materials (Sutton, 2001), and norms (Davenport and Prusak, 1998). In epistemological terms, knowledge can be defined as 'justified true belief' emerging from experiencing, reflection and inference processes (Nonaka, 2002; Audi, 2003). Knowledge is derived in human minds through understanding and justification and becomes objects-related through human action processes (Jones, 1964; Davenport and Prusak, 1998; Nonaka, 2002). In other words, knowledge is a result of understanding (Varela, Evan and Rosch, 1995; Garud, 1997).

Broadly, knowledge can be classified into two distinct types-explicit and tacit knowledge. Explicit knowledge is more concerned with documentation and codifying (Nonaka and Takeuchi, 1995). It can be transmitted in formal systems. Explicit knowledge is easy to capture in records, such as databases and archives, and accessed on a sequential basis (Nonaka, 2002). Varela and colleagues (1995) consider explicit knowledge as 'knowledge that' or propositional knowledge which is knowledge at the state of thinking and may not be done as thinking (Ryles, 1949). Sutton (2001) deems that explicit knowledge is not sufficient to enable effective performance. It needs to be put into practice in order to create the particular ability and skill - procedural knowledge (Ryle, 1949; Brown and Duguid, 1998).

Tacit knowledge, on the other hand, is deeply located in action, commitment, and involvement in a specific context (Nonaka, 2002). Tacit knowledge is normally referred to know-how which resides in human heads, skills and actions (Newell, Robertson, Scarbrough and Swan, 2002), work practice (Brown and Duguid, 1998) and organisational routines (Badaracco, 1991). Indeed, tacit knowledge is not referred to only as procedural knowledge. Nonaka (2002) enumerates that tacit knowledge involves both technical and cognitive elements. Technical elements of tacit knowledge include concrete know-how, crafts and skills. The cognitive elements are 
concerned with the mental model emerged in human minds. They include schemata or theories, paradigms, beliefs and viewpoints to perceive and define their world. Nonaka (2002) states that the cognitive elements of tacit knowledge are important factors for creating new knowledge. This is because tacit knowledge comprises subjective insights and intuitions (Nonaka and Takeuchi, 1995).

On this basis, explicit knowledge can be classified to be 'knowledge that' or 'knowing that' which is considered declarative knowledge from the perspective of an organisation's operation. Whereas, tacit knowledge can be classified into procedural knowledge or know-how, theoretical knowledge or know-why, and personal knowledge or know-who. The technical elements of tacit knowledge can refer to procedural knowledge while the cognitive elements of tacit knowledge are focused on theoretical knowledge which is discussed in the next section.

In an organisational context, knowledge comprises valuable things that are available in the performance of organisational activities. It involves organisational members and actions (Pan and Scarbrough, 1999). Thus, knowledge comprises true beliefs, values, insights, experiences, understanding and skills of individual and organisations. This research will use the clear definition from Davenport and Prusak (2002, p.5) to describe knowledge in community enterprises.

Knowledge is a fluid mix of framed experience, values, contextual information, and expert insight that provides a framework for evaluating and incorporating new experiences and information. It originates and is applied in the minds of knowers. In organizations, it often becomes embedded not only in documents or repositories but also in organizational routines, processes, practices, and norms.

Organisational knowledge is concerned with core competencies which encompass declarative knowledge or know-what knowledge-explicit knowledge, and the particular abilities_-procedural knowledge or know-how knowledge which focuses on practising and collective (Brown and Duguid, 1998). 


\subsubsection{Knowledge types}

In order to extend the understanding of organisational knowledge of community enterprises, the types of knowledge will be considered specifically within the perspective of operational systems, and can be distinguished into four types: declarative knowledge, procedural knowledge, theoretical knowledge (Sanchez, 1997), and personal knowledge (Van Den Bosch and Van Wijk, 2001).

\subsubsection{Declarative knowledge}

Declarative knowledge is referred to as know-what knowledge (Nahapiet and Ghoshal, 1998). It is knowledge at the level that organisations possess and describe, but it does not ensure that organisations can utilise such knowledge for business operations. In a sense, declarative knowledge is considered strategic knowledge which comprises purposes and available knowledge of organisations (Sanchez, 1997; Van Den Bosch and Van Wijk, 2001) to accomplish that which is concerned with fact and propositions (Moorman and Miner, 1998; Nahapiet and Ghoshal, 1998). As mentioned previously, 'knowledge that' or declarative knowledge needs to be put into practice in order to create particular abilities and skills_-procedural knowledge (Ryle, 1949; Varela et al., 1995; Brown and Duguid, 1998). On this basis, declarative knowledge refers to explicit knowledge which is easy to capture and transfer (Nonaka, 2002). Thus, declarative knowledge can be found in organisations' documents, records and formal activities and is considered a crucial resource for knowledge creation, especially procedural knowledge creation.

\subsubsection{Procedural knowledge}

Procedural knowledge or know-how is practical knowledge which is concerned with understanding and the ability to perform activities (Sanchez, 1997). Procedural knowledge is concerned with well-practised skills and routines over a period of time (Sanchez, 1997; Nahapiet and Ghoshal, 1998; Skyrme, 1999; Garud, 1997) using 'knowing that' or declarative knowledge (Ryle, 1949). Procedural knowledge of an organisation is derived from organisational routines, practice (Garud, 1997), experience, organisational culture (Brown and Dugid, 1998; Verela et al., 1995) and learning by doing (Sanchez, 1997). It is considered usable knowledge as it is the practical understanding that enables organisations to perform activities, to operate production and services (Verela et al., 1995). It is unique for each organisation and it 
can be of competitive advantage in creating better and different services and products than competitors (Sanchez, 1997. It is arguable that procedural knowledge forms the technical elements of tacit knowledge, which is difficult to lose (Sanchez, 1997), capture and transfer (Van Den Bosch and Van Wijk, 2001). Procedural knowledge is normally located in human heads, organisational procedures and routines (Despres and Chauvel, 2000).

\subsubsection{Theoretical knowledge}

Theoretical knowledge or know-why knowledge (Sanchez, 1997) is concerned with the understanding of principles underlying the performance of activities (Garud, 1997). In other words, theoretical knowledge is concerned with the reasons for performing activities (Badaracco, 1991). Similarly, Sackmann (1991) calls the reasons about why to do, choose, prefer, and deal with things and people, as 'axiomatic knowledge'. Sackmann states that axiomatic knowledge is based on the fundamental beliefs of an organisation. Choo (1998) deems that knowledge based on shared assumptions and beliefs refers to cultural knowledge. Similarly, Holsapple and Joshi (1999) consider culture as a part of knowledge. They call it schematic knowledge, which can inform the behaviour of organisational members. In a sense, from the perspective of epistemology, theoretical knowledge refers to moral knowledge which is concerned with moral judgments that fit with cultural conditions (Audi, 2003). On this basis, theoretical knowledge includes know-why knowledge, axiomatic knowledge, cultural knowledge and moral knowledge. Thus, theoretical knowledge enables organisations to make decisions based on their situations and environment (Sanchez, 1997) and allows organisational functions to run smoothly (Bosch and Wijk, 2001). According to Nonaka (2002), theoretical knowledge can be viewed as tacit knowledge in the form of cognitive elements. This is because theoretical knowledge encompasses shared values of organisations and ethical principles which are cognitive elements of tacit knowledge.

\subsubsection{Personal knowledge}

Personal knowledge or know-who knowledge is concerned with knowing who has the knowledge or ability to perform particular work in an organisation (Skyrme, 1999; Bosch and Wijk, 2001). Personal knowledge can be referred to as personal identity which is concerned with the identification of idiosyncratic characteristics-bodily 
attributes, abilities, psychological traits and interests (Ashforth and Mael, 1989). According to the social and group identity concept of Tajfel (1978), and Ashforth and Mael (1989), people consider themselves as belonging to a society and group by comparing their identities with those of others. This context allows members in society to identify the abilities and interests of individuals or the know-who of an organisation. This is similar to transactive memory-locating knowledge of the abilities of organisational members-mentioned by Dooley, Corman and McPhee (2002). According to Wegner (1987), transactive memory can be viewed as personal knowledge. Wegner (1987) points out that transactive memory is considered as an organisational directory that indicates locations of knowledge. In a sense, personal knowledge is a pointer that leads to the sources of organisational skills and abilities Personal knowledge can embed in human heads and organisational routines and procedures, which refers to tacit knowledge, whereas personal knowledge in the forms of organisational storage, such as directories and databases, can be referred to as explicit knowledge.

\subsubsection{Particular knowledge elements}

Generally, enterprises require knowledge from several sources including customers, suppliers, competitors, and researchers (Choo,1998). According to their unique characteristics, CEs would be associated with indigenous knowledge and particular knowledge elements from a range of sources. KMI (2005a) notes that people organisations require knowledge from sources including local experts, support agencies and scholars in the forms of both documents and local wisdom. Those knowledge elements include experience, wisdom, creativity, and collective senses and shared values.

\subsubsection{Indigenous knowledge}

As discussed in section 2.1, CEs are concerned with grassroots, sustainable development and local businesses. They pay attention to local resources including materials, human resources, human capital and social capital. In Thailand, most CEs employ the local skills and wisdom of members (Walaisatien, 1996; Jommuang, 1997), friends, NGOs, and not-for-profit consulting firms (Barton, 1997) for their business operation. As a result, CEs are concerned with indigenous knowledge (IK). 
To extend the understanding about organisational knowledge of CEs, this section will discuss the characteristics of indigenous knowledge and systems.

To begin with, IK refers to traditional and local knowledge possessed by groups of people living in a particular area for a long period of time (Rao, 2006; Langill, 2007). IK is not limited only to aboriginal people (Storey, 2005), and not concerned with oldfashioned or static knowledge (Langill, 2007). It is dynamic and continues developing over time (Obomsawin, 2000; Hansen and VanFleet, 2003). Furthermore, it is collective, experiential and subjective (Sefa Dei, Hall and Rosenberg, 2000), holistic, and implicit (Obomsawin, 2000).

First, IK is collective and dynamic and unique in nature. It represents local knowledge accumulated over generations. It is dynamic in nature, because it is the integration of historical knowledge and new knowledge. It is derived through the collaboration and interaction of people and the environment (Obomsawin, 2000; Sefa Dei, Hall and Rosenberg, 2000; Hansen and VanFleet, 2003). IK is unique to a particular group culture and environment, because it is involved with long-term occupancy in a particular area together with adaptation to local conditions, including culture, environment and local people's requirements for living (Sefa Dei, Hall and Rosenberg, 2000; Hansen and VanFleet, 2003; Langill, 2007). Furthermore, IK is captured through observation and justification by local people in a natural environment and particular social phenomena (Waldram, 1986).

Second, IK is rooted in personal and direct experience, and associated with long-term understanding of local environment and culture (Sefa Dei, Hall and Rosenberg, 2000). It is concerned with first-hand experience (Obomsawin, 2000; Storey, 2005). It is captured by observation because it is repeating, occurring and revealing through intuitive vision (Castellano, 2000) and the philosophic and cognitive systems of local experts (Obomsawin, 2000). As a result, local knowledge is subjective in nature.

Third, IK is holistic. It is derived from the integration of complex components including individual components, local ecosystems and social context. Individual components comprise experience, know-how, wisdom, insights, creativity, perceptions, innovative capabilities (Obomsawin, 2000). Social context focuses on 
belief and spiritual insights of the local community (Castellano, 2000; Storey, 2005). IK does not make sense when it is isolated from social context and local environment (Sefa Dei, Hall and Rosenberg, 2000; Castellano, 2000).

Lastly, IK is implicit and requires traditional methods for capture and transfer. It encompasses the skills, experience and insights of people (Obomsawin, 2000; Rao, 2006). It is located in people's minds, activities, community practices and culture, such as community values, beliefs, rituals and traditional songs and stories (Korma, 1995; Grenier, 1998). IK plays a crucial role for local culture survival and is related to certain values and moral codes (Storey, 2005). It passes on to generations through traditional methods - oral transmission, teaching and learning from community elders, and direct experience (Kroma, 1995; Sefa Dei, Hall and Rosenberg, 2000; Storey, 2005).

On this basis, IK is more concerned with tacit and procedural knowledge. It is focused on occupancy and practice. It is difficult to encode and capture through conventional methods and formal education. It can be referred to as cultural and moral knowledge because IK comprises social, political, economic and spiritual aspects of the local way of life (Langill, 2007). It emerges under investigation of a cultural context (Langill, 2007), according to Kroma (1995) and Grenier (1998), can be found as encoded knowledge in the forms of proverbs, stories, riddles, music and songs.

From the perspective of indigenous people and eastern thought, IK is known as local wisdom, which Small (2004) defines as indigenous people's ability to create accumulated knowledge and the capacity for making judgments for the creation of a good life. In Thailand, local wisdom is referred to as knowledge integrated with indigenous knowledge, ethical principles and culture, and adapted to be appropriate for local societies, environment and culture (Thai Knowledge Centre, 2007). It can be seen in local tales, proverbs, and songs the forms of books, palm leaf books, paintings on temple walls (Kotkanta, 2007). CEs can be influenced by the IK derived from the local area, because CEs rely on local resources including knowledge and human capital. 


\subsubsection{Experience}

Experience refers to what we absorb from various phenomena and activities, and what we have done and what has happened to us (Davenport and Prusak, 1998). In one sense experience is derived from observations that do not involve direct experience. In a second sense observations are experiences that focus on sense making and judgment without direct experience. Meanwhile, the experience in the later sense of Davenport and Prusak (1998) can be viewed as true experience which is what people see and hear by themselves without judgement (Kitaro, 1960). It is derived with doing (Lam, 2000). According to the nature of knowledge of Ryle (1949), Varela et al. (1995), and Brown and Duguid (1998), observation can be viewed as declarative knowledge - knowledge at the state of knowing and thinking. However, it is a crucial resource for procedural knowledge creation (see section 2.2.2.2). Meanwhile, true experience can be viewed as procedural knowledge. This is because direct experience allows people to perform activities and can guide people in doing things. As mentioned previously in this section, direct experience is a crucial element of procedural knowledge and IK creation.

\subsubsection{Creativity}

Creativity is defined as original ideas and specific domain knowledge (Kilgour, 2006). Amabile (1983) deems that creativity is derived from a combination of task motivation, domain skills_relevant knowledge, technical skills and talents, and creativity skills including cognitive style, heuristics and working styles. Similarly, Sternberg and Lubart (1991) mention that creativity underlies certain knowledge areas. Creativity is normally generated by people who have a certain level of knowledge in the field. In the organisational context, domain skills, especially relevant knowledge, include organisational and customer capital which are concerned with the context of the organisation (Thorbjornsen and Mouritsen, 2005). Customer capital refers to a type of intellectual capital that is concerned with the value of the organisation's relationship with customers or knowing about customers and treating customers as assets (Stewart, 1997). It is arguable that organisational and customer capital support the embodiment of creativity as a valuable strategy for organisations. According to the nature of knowledge discussed by Ryle (1949), Varela et al. (1995), and Brown and Duguid (1998), creativity refers to declarative knowledge. However, creativity can be also considered as the ability to both problem solve and create new 
scientific findings, new inventions and new movements (Sternberg and Lubart, 1996). Similarly, Woodman, Sawyer and Griffin (1993) define creativity in the organisational context as valuable creations, and useful new products, services, ideas, procedures and processes. As a result, the creativity in this sense can be viewed as procedural knowledge. This is because creativity in this context refers to usable knowledge for performing work. According to procedural knowledge characteristics, procedures and processes come with practical engagement and organisational routines. This is consistent with the statement of Sternberg and Lubart (1991), who deem that creativity is subjective and depends on the environment. A different environment would provide different ideas.

\subsubsection{Collective senses and shared values}

Sense refers to sympathy which is concerned with making the correct perception of what is reasonable or having the correct sense to arrive at what is true (Aristotle, /2002). Aristotle considers good sense to be a virtue that is a part of wisdom, extraordinary scope of knowledge and deeply rooted in culture and sound judgment (Baltes, 1993). In an organisational context, collective senses refer to organisational culture. Gopalakrishnan and Santoro (2004) consider the sense of involvement, which refers to the sense of ownership, responsibility and commitment, as organisational culture. Organisations with a strong sense of involvement or sense of ownership and adaptability can enhance members' motivation in terms of involvement with new technology (Denison and Mishra, 1995) and willingness to share knowledge (Gopalakrishnan and Santoro, 2004). In a sense, collective senses can be viewed as the principles and reasons behind decision-making for performing activities. According to the characteristics of theoretical knowledge described by Garud (1997), senses and shared values are referred to as theoretical knowledge.

\subsection{Knowledge management}

This section focuses on the concept of knowledge management (KM) and its components including KM approaches, KM for CEs, and knowledge processes.

\subsubsection{Knowledge management approaches}

Knowledge management is defined as a conceptual framework of activities and perspectives in order to gain, deal with, and benefit from the corporation's knowledge 
assets (Wiig, 1994). Grant (1996) states that KM in organisations focuses on the utilisation of knowledge in order to create value by creating, acquiring, storing and deploying knowledge for products and services. Several researchers including Davenport and Prusak (1998), Gamble and Blackwell (2001), and Al-Hawamdeh (2003) assert that KM encompasses the processes of the knowledge cycle: identifying, organising, developing, and exploiting knowledge. However, many researchers believe that knowledge has abstract and subjective elements (Huizing and Bouman; 2002; Sveiby and Simons, 2002; Christensen and Bukh, 2005), and may not be managed, unlike data and information (Huizing and Bouman; 2002; Rasheed, 2005). Nevertheless, some researchers-Balasubramanian, Nochur, Henderson and Kwan (1999) — consider knowledge to be an object which can be managed as a module or packet.

Regarding the perception of knowledge, $\mathrm{KM}$ can be considered under two approaches: artefact-oriented approach and process-oriented approach. The artefactoriented approach focuses on system theory, information processing and use of information technology for codification of knowledge. From this perspective, knowledge is considered to be a visible product which can be codified and stored in computer systems. The process-oriented approach, on the other hand, focuses on knowledge creation. From this perspective, knowledge is managed through social interaction and requires a combination of humans and technology. Knowledge is perceived as a subjective, action-oriented and dynamic matter (Christensen and Bukh, 2005).

Similarly, Hansen, Nohria and Tierney (1999) consider KM in terms of two approaches - codification and personalisation. The codification approach focuses on codifying, storing and reusing knowledge through a 'people-to-document' method in which knowledge is created into forms that are easy to distribute and to share. In a sense, Sveiby (2001) considers this context as IT-track KM, which is involved with the construction of information management systems. Thus, KM in this context can be seen as an artefact-oriented approach. Meanwhile, the personalisation approach refers to the process-oriented approach. The personalisation approach focuses on personal contact. Knowledge is created and shared through a 'people-to-people' method (Hansen, Nohria and Tierney, 1999). The KM in this context occurs through the 
communication of people, socialisation, networking and mentoring (Christensen and Bukh, 2005). This KM refers to people-track KM, which involves learning and managing competencies (Sveiby, 2001).

Most KM takes a technological approach which is based on information technology to support knowledge sharing and codifying (Scarbrough and Carter, 2000; Alavi and Leidner, 2001; Hislop, 2005). This is because KM originated in large organisations that depend on technology (Prusak, 2001). With regard to its objectives-generating organisational knowledge assets or intellectual capital and leveraging knowledge to gain the most benefit for organisations- $\mathrm{KM}$ requires several factors, including knowledge processes, technology (Earl, 1997; Balasubramanian, Nochur, Henderson and Kwan, 1999; Lee and Kim, 2001; Dalkir, 2005), and organisational structure or social activities (Balasubramanian, Nochur, Henderson and Kwan, 1999; Dalkir, 2005).

This study will consider KM based on the process-oriented approach which focuses on knowledge which is created through a combination of humans and technology. It is arguable that this KM approach would be appropriate for micro and community enterprises that do not rely on information technology (see section 2.1). KM based on this approach would consist of four main components: knowledge processes, organisational culture, organisational management, and appropriate technologies which are focused on the characteristics and constraints of CEs. Each component will be discussed below.

\subsubsection{Knowledge management for micro enterprises}

Given the characteristics and limits of MEs and CEs, it is argued that KM for CEs focuses on the process-oriented approach together with the personalisation approach. KM for MEs and CEs is not merely a smaller scale model of KM for large enterprises as suggested by Hylton (2002). Most KM for large enterprises focuses on ICTs (Sparrow, 2001), while MEs and CEs may have limited ICTs. KM for small firms focuses on informal knowledge processes (Wong and Aspinwall, 2004) with the use of human potential (Chattell, 1998), intellectual capital and social capital (Sparrow, 2001). This is because MEs and CEs have small scale organisations and flat organisational structures, informal operational systems (Wong and Aspinwall, 2004; 
Sparrow, 2005), organic organisational culture (Wong and Aspinwall, 2004; Rasheed, 2005), local resources and social capital (Sparrow, 2001), and a low level of technology (Sparrow, 2001; Martin and Matlay, 2003). Small enterprises tend to focus on informal communication and pay less attention to formal knowledge process (Wong and Aspinwall, 2004). Nevertheless, most research for KM for small enterprises focuses on KM for SMEs. As discussed previously, MEs and CEs have specific characteristics which are different from the characteristics of SMEs. Therefore, CEs require specific KM characteristics that are different from KM of SMEs.

\subsubsection{Knowledge processes}

According to the definition of KM, knowledge processes involve the processes of emerging in human minds, creating into explicit forms, obtaining knowledge from others, circulation and utilisation for the better performance of the organisation. KM researchers assert that knowledge processes comprise many activities. Skyrmy (1999) states that knowledge processes comprise three main processes-knowledge conversion, innovation and sharing. The innovation process is concerned with knowledge creation and codification, whereas the sharing process is focused on knowledge capture, sharing and exploitation. Similarly, Dalkir (2005) states that KM encompasses the processes of creation, capture, codification, sharing, accessing, application and reuse of knowledge. In order to obtain the full potential of KM, the processes should comprise knowledge transfer (Gupta, Sharma and Hsu, 2004), and knowledge verification or testing (Bhatt, 2001; Collison and Parcell, 2004). Therefore, this research considers seven processes-creation, capture, codification, sharing, transfer, verification and utilisation. Furthermore, the knowledge processes are not cyclical and sequential. They are randomly recursive as can be seen from figure 2.1. 


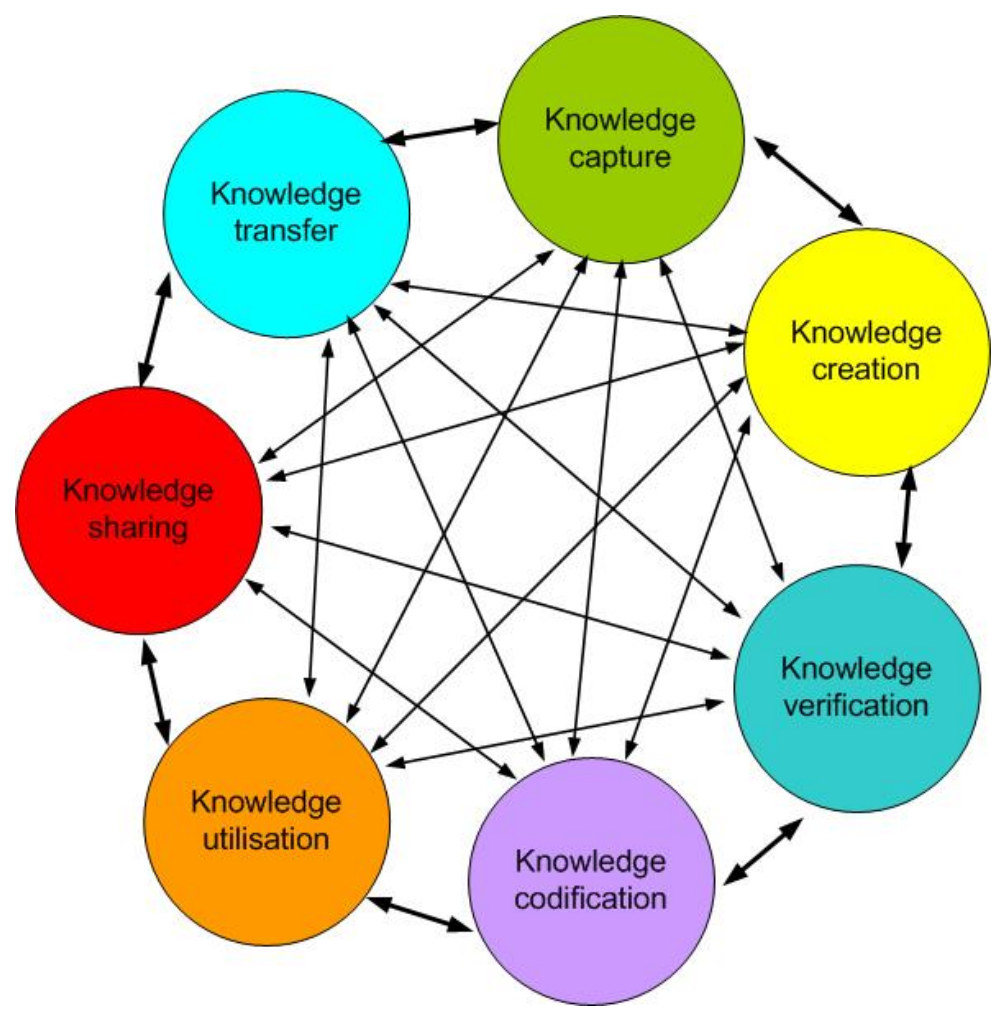

Figure 2.1 The randomly recursive knowledge processes.

\subsubsection{Knowledge creation}

Fundamentally, knowledge is created in human minds as an abstract element or tacit knowledge, and transformed into objects or explicit knowledge. Knowledge creation involves generating and discovering new knowledge of any types (Al-Hawamdeh, 2003), and covers the conversion of tacit knowledge into explicit knowledge and vice versa (Nonaka, 1994; Sena and Shani, 1999). Nonaka (1994) suggests the model of knowledge conversion has four modes: socialisation, externalisation, internalisation and combination. Socialisation is more concerned with tacit knowledge sharing and creation, while combination focuses on information processing that provides knowledge in codified or concrete forms. Externalisation is concerned with tacit knowledge capture and its transfer to explicit knowledge. Internalisation, on the other hand, is focused on the conversion of explicit knowledge into tacit knowledge through organisational learning, especially learning by doing. In a sense, internalisation is concerned with tacit knowledge creation by utilising explicit knowledge. AlHawandeh (2003) considers internalisation to be more than a process of developing 
know-how or individual skills, and experience. It is also the process of contextualising explicit knowledge through a sense making process.

On this basis, knowledge creation is focused on tacit rather than explicit knowledge creation and on the integration of individual knowledge into organisational knowledge in forms available for organisational activities (Nonaka, 1994). Nonaka points out that organisational knowledge creation encompasses the processes of individual knowledge enlargement, amplification, crystallisation, justification and networking knowledge. However, the organisational knowledge creation model of Nonaka can be viewed as several other knowledge processes. Knowledge enlargement is concerned with knowledge capture, amplification is associated with knowledge sharing, and justification focuses on knowledge verification. Meanwhile, networking knowledge is concerned with knowledge codification and knowledge distribution. Each process will be discussed in the following sections.

For knowledge generation or crystallisation, knowledge creation can be classified into three approaches - practical, educational and socialised approaches. First, the practical approach is concerned with knowledge creation by doing, work processes which are likely to be informal (Al-Hawamdeh, 2003), and problem solving processes (Baek, Liebowitz, Prasad and Granger, 1999). Nonaka (1994) states that the processof problem-finding and solution-creation is referred to as an innovation process, which is a key process for knowledge creation. 'Doing a job' allows people to obtain true experience. This is considered procedural knowledge creation (Lam, 2000). Nonaka (1994) states that doing plays a crucial role in the process of knowledge crystallisation or organisational knowledge embodiment. Furthermore, according to Trice and Beyer (1993), ‘doing a job’ can be considered a sense-making behaviour process. Events and actions allow people to make sense, because people normally think about their actions after they perform them. Thus, the practical approach focuses on procedural and theoretical knowledge creation.

Second, the educational approach focuses on competency building (Handy, 1995) that is considered tacit knowledge creation (Al-Hawamdeh, 2003). As discussed previously, internalisation-creating tacit knowledge from explicit knowledgefocuses on learning by doing. Nonaka (1994) states that people share explicit 
knowledge in action and transfer to the process of trial-and-error leading to the emergence of particular tacit knowledge. Learning processes support the cognitive elements of tacit knowledge creation and the cognitive process of sense making which is focused on knowing and perceiving (Trice and Beyer, 1993, pp. 18-20). Informal learning is very important for tacit and procedural knowledge creation. Indeed, it is a very important process for indigenous knowledge creation.

Finally, the socialised approach focuses on interaction. As mentioned previously, socialisation is a mode of tacit knowledge sharing and creation. Social interaction between individuals or "communities of interaction" plays an important role in generating new knowledge (Nonaka, 1994). This approach is concerned with cognitive elements of tacit and theoretical knowledge creation rather than know-how creation.

\subsubsection{Knowledge capture and codification}

Capturing knowledge means obtaining knowledge from various sources and in many forms, and also involves organisation and codification (Holsapple and Joshi, 1999; Al-Hawamdeh, 2003; Dalkir, 2005). Similarly, Balasubramanian, Nochur, Henderson and Kwan (1999) consider knowledge capture to be the process of collecting and interpreting information from both internal and external sources, and organised into explicit forms for utilisation. It is clear that this process can be divided into two processes: capture and codification. Knowledge capture focuses on acquiring knowledge, whereas, knowledge codification focuses on organising knowledge to become encoded knowledge.

There are many knowledge capture techniques including training, education, apprenticeships, lesson-learned programmes, environmental scanning and licensing (Wiig, 1999). Knowledge capture activities can be grouped into three approaches in the same way as knowledge creation. First, with the practical approach, knowledge capture occurs through work processes. Furthermore, the practical approach of knowledge capture includes licensing (Wiig, 1999) and acquiring experts who possess particular knowledge (Gupta, Sharma and Hsu, 2004). Second, the educational approach is concerned with training programmes, apprenticeships, lessons learned, environmental scan (Wiig, 1999) and document study (Garza and Ibbs, 1992). Finally, 
the social approach includes discussion and the interaction of individuals. According to the social capital theory of Coleman (1988), social connection and social relations provide information channels that allow members of societies to transfer and capture knowledge. Similarly, Ryu, Kim, Chaudhury and Rao (2005) suggest that an organisation can acquire knowledge through three methods: learning by doing, learning from others and learning by investment. Learning by doing and learning by investment are concerned with education programmes. Meanwhile, learning from others relates to communities of practice ( $\mathrm{CoP}$ ) which comprises members in the community sharing and learning from each other. Garza and Ibbs (1992) identified three effective methods of acquiring knowledge in the construction field. These are document review, observation and expert interviews, which are associated more with the educational and socialised approaches. Meanwhile, Hylko (2005) affirms that one effective form of knowledge capture is discussion forums, called 'the critical decision method' which is concerned with the socialised approach.

Recently, the term 'knowledge engineering' has been applied to the knowledge capturing process. Several authors (Wiig 1999; Liebowitz 2001; Dalkir 2005) have argued that knowledge acquisition techniques used for expert systems development can be applied to knowledge capture. Knowledge acquisition techniques are concerned with extracting knowledge from experts, including people and other sources, which is then stored or coded in knowledge-based systems according to a rule-based approach (Durkin, 1994). However, knowledge engineering techniques may not be not suitable for CEs because they are unlikely to be able to afford to pay for knowledge engineers and their tools. Furthermore, CEs rely on local knowledge which relies on traditional methods, such as observation with particular environment of knowledge and learning from experts (see section 2.2.3.1).

Knowledge organising and codification, generally, entail content management which is concerned with classification or information architecture, authoring, and indexing and searching. Content management will be discussed more in section 2.3.5.

\subsubsection{Knowledge sharing}

Knowledge sharing is the transfer of knowledge between people or organisations (Keong and Al-Hawamdeh, 2002). It can occur through both formal and informal 
processes (Reid, 2003; Caulkins, 2004). Formal activities are more focused on explicit knowledge sharing, whereas informal activities are focused on tacit knowledge sharing. This is because explicit knowledge can be shared in a straightforward manner (Hislop, 2005). On the other hand, given the characteristics of tacit knowledge, its transfer requires socialisation and acculturation (Blackler, 2002), which encourage organisation members to share their skills and learn new skills from each other. Harris and Moran (1996) state that the acculturation process requires collaboration and learning to obtain and to adapt new skills, and gain familiarity. Tacit knowledge can be effectively transferred through the informal interactions of in-groups (Scarbrough and Carter, 2000; Steinheider and Al-Hawamdeh, 2004; Mitchell, 2005), and through work processes (Scarbrough and Carter, 2000; Ford and Chan, 2003). Similarly, Wenger, McDermott and Snyder (2002) deem that a CoP is an effective method, especially for tacit knowledge sharing. This is because a $\mathrm{CoP}$ is a group of organisational members informally joining together in order to expand their knowledge and develop their capabilities.

Whether knowledge sharing occurs through formal or informal processes, it is clear that knowledge transfer relies on receivers who need and are willing to receive knowledge from other agents, and providers who are willing to transfer or review and evaluate their knowledge. Effective knowledge transfer methods depend on many factors, including organisational structure and management, organisational environment, organisational culture, and technology (Davenport, De Long and Beers, 1997; Keong and Al-Hawamdeh, 2002). The effective factors will be discussed in the next section and the section on support components for KM.

\subsubsection{Knowledge transfer}

Knowledge transfer is concerned with knowledge flows or dissemination to other parts of the organisation or other organisational members (Gupta, Sharma and Hus, 2004). It is similar to knowledge sharing, but knowledge transfer is focused on oneway knowledge flow and interpretation of the captured knowledge. Knowledge transfer comprises two processes-communication and interpretation. The communication process is concerned with information flow to receivers through appropriate media. Meanwhile, the interpretation process focuses on applying, 
accepting, assimilating, and utilising the knowledge for organisational routines (Albino, Garavelli and Schiuma, 1999).

The knowledge transfer can take two approaches - a tool-centred approach and a human-centred approach. The tool-centred approach focuses on use of ICT to transfer explicit knowledge. Meanwhile, the human-centred approach is concerned with techniques to transfer tacit knowledge (Carrillo, Robinson, Anumba and Bouchlaghem, 2006). According to the KM approach of Hansen, Nohria and Tierney (1999), the tool-centred approach of knowledge transfer refers to codification in which knowledge is transferred through a 'people-to-document' method. The humancentred approach, on the other hand, refers to a personalisation approach through which knowledge is transferred based on interaction, socialisation and learning processes. From the indigenous knowledge (IK) perspective, knowledge transfer is focused on the human-centred or personalisation approach, because IK is tacit, collective, experiential, subjective and holistic (see section 2.2.3.1). Many researchers note that tacit knowledge requires direct communication for transfer (Lam, 1997; Connell, Klein and Powell, 2003; Jasimuddin, 2007). Many factors encourage the personalisation approach of knowledge transfer, including collaborative environment, learning capacity, contextual assumption addressing and embodying of knowledge in tools (Connell, Klein and Powell, 2003).

Generally, the effectiveness of knowledge transfer depends on factors including rich transmission channel, absorptive capacity, motivation, value of knowledge (Gupta and Govindarajan, 2000b), openness and trust (Wathne, Roos and Von Krogh, 1996). Gupta and Govindarajan (2000b) affirm that organisation socialisation mechanisms can enhance the richness of communication channels, because interpersonal familiarity and relationship can increase the openness of communication. Wathne, Roos and Von Krogh (1996) consider these as open social contexts, and that face-toface communication is the richest medium for knowledge transfer.

Openness is concerned with the willingness of the giving party to share knowledge, which depends on the quality of dialogue, and the degree of interaction or knowledge shielding (Wathne, Roos and Von Krogh, 1996). Furthermore, the degree of openness 
depends on the level of trust, which is based on beliefs about the predictability of the transfer partners’ positive response.

Absorptive capacity is the ability to recognise the value of new knowledge, assimilate it and apply it to the work process (Cohen and Levinthal, 1990). This capacity depends on the level of prior related knowledge or familiarity with the incoming knowledge, and the homophily of givers and receivers (Gupta and Govindarajan, 2000b). Homophily is defined as the degree of similar attributes, such as beliefs, education, social status and interests (Rogers, 1995). Previous experience and knowledge play a vital role for the interpretation process (Albino et al., 1999). This is because prior knowledge can enhance learning, which is cumulative and at its greatest when related to pre-existing concepts (Cohen and Levinthal, 1990).

The motivation for knowledge transfer is focused on incentive systems rewarding eagerness to learn and help others (Gupta and Govindarajan, 2000b). Motivation is more concerned with needed knowledge creation which can initiate knowledge transfer (Kwan and Cheung, 2006). However, many researchers argue that extrinsic reward and incentive systems can reduce the willingness of organisational members to share their knowledge, because intrinsic motivation is derived from enjoyment in doing tasks and self-actualisation needs (Gupta and Govindarajan, 2000b; Bartol and Srivastava, 2002; Smith and McKeen, 2005). Motivation and reward systems can be both intrinsic and extrinsic systems, which will be discussed in section 2.3.4.

The value of knowledge is concerned with perception of the benefit of knowledge gained. Gupta and Govindarajan (2000b) consider the value of knowledge transferred in terms of the relative advantage and relevance of knowledge needed. In terms of knowledge transfer between organisations, the size of the knowledge source and relative economic level of parties can affect knowledge transfer effectiveness. It is arguable that if the target party realise the relevance of knowledge and advantages for their performance, they will be eager to obtain the knowledge.

\subsubsection{Knowledge verification}

Not all available knowledge in organisations is appropriate or ready for utilisation. Bhatt (2000) asserts that knowledge development for organisations also encompasses 
review and revision processes in order to verify knowledge. Knowledge verification is the process of testing and adapting particular knowledge based on the situation in order to be suitable for the organisation. Knowledge testing and verification can occur through many activities including organisational member debates (Weick, 1979; Collison and Parcell, 2004), use of knowledge and reflection (Choo, 1998; Collison and Parcell, 2004). Weick (1979) states that raw data that is critiqued and contemplated will be found meaningful for organisations. Use and reflection refer to the processes of applying knowledge to particular situations in order to provide feedback that would benefit other parts of the organisation. In a sense, this can be viewed as an evaluation process to verify the appropriateness of new knowledge for organisational goals. On this basis, such knowledge verification would be appropriate to CEs and their knowledge types. As noted previously, the CEs are community based, thus knowledge circulation in groups and group debate would be a common work style for them. Meanwhile, the use of knowledge and reflection match with indigenous knowledge systems.

\subsubsection{Knowledge utilisation}

Knowledge utilisation is concerned with using and applying knowledge to work processes or business functions. Alavi and Leidner (2001) and Dalkir (2005) consider knowledge utilisation to be the application of knowledge, using computer-based tools which allow organisation members to access knowledge through KM systems. However, KM applications alone cannot ensure that knowledge will be applied to business operations or products and services. Knowledge exploitation is concerned with the process of gaining benefit for organisations by transferring knowledge from sources such as a research and development division to production and customer services (Zack, 2002). Similarly, Holsapple and Joshi (1999) consider knowledge utilisation as the process of applying existing knowledge in order to generate new knowledge and produce externalisation. In a sense, knowledge utilisation is concerned with applying knowledge possessed in the organisation to perform activities that can be seen to have explicit results, such as products, services, procedures and regulations.

Knowledge application and exploitation require a demand driven approach which encourages organisation members to use and share knowledge (Swan, Newell, Scarbrough and Hislop, 1999). The utilising combines technological, operational and 
social aspects because technology can enable and facilitate access to knowledge repositories (Davenport and Prusak, 1998; Sena and Shani, 1999; Reid, 2003; AbuRashed, Bertaux and Okunoye, 2005), while the operational and social aspects can involve exploitation of knowledge to achieve business goals (Zack, 2002). These factors will be discussed below.

CEs tend to focus on IK, which relates to skills and experiences. As mentioned previously, IK is dynamic in nature. It is derived from interaction and adapted to suit the nature and environment of societies for the survival of the local people. It is accumulated over generations through observation and oral transmission (Kroma, 1995; Storey, 2005). As a result, knowledge creation of CEs focuses on implicit knowledge creation and sharing through practical and collaborative approaches. IK capture requires socialisation and participatory techniques to express individual experiences and understand the experiences of others (Francis, 2006). For knowledge codification, organisation using technologies is necessary to derive encoded and explicit knowledge. Appropriate technologies and processes have to be considered. This topic will be discussed in section 2.3.5. Because local people often have difficulty using computers and gaining access to the Internet (Sukula, 2006), knowledge utilisation in CEs tends to focus on operational and social aspects rather than technologies.

\subsubsection{Organisational culture}

As mentioned previously, organisational culture can affect knowledge processes. CEs are community-oriented, therefore, their organisational culture is influenced by community culture. As a result, this section will start by examining national culture and Thai culture because Thailand is the location of the research site in this study. Then organisational culture and knowledge driven culture will be discussed.

\subsubsection{National culture}

Culture can be defined as the patterns of acting, thinking, and feeling that bind social members together and guide them to perform and react in societies (Hofstede, 1980). Kluckhohn (1962, p.25) also defines culture as 
“...a way of thinking, feeling, believing. It is the knowledge stored up (in memories of men, in books and objects) for future use-patterns for doing certain things in certain ways, not the doing of them.”

Kluckhohn (1962) mentions that culture comprises traditions, ideas and values. Schein (1992) views culture as having three aspects: artifacts, espoused values, and basic assumptions. Artifacts are culture in forms that can be seen, heard, and felt. Espoused values include beliefs and ideas, and the ways people behave. Finally, basic assumptions are invisible elements, which provide guidelines for how people think and feel about things and react to situations.

Culture is created naturally with the consensus of society (Antony and Gales, 2003) and over time becomes norms and standards (Hampden-Turner, 1990) which can guide people in living together harmoniously, solving problems, and predicting the behaviour of others (Kluckhohn, 1962). Differences between cultures are commonly considered in terms of the five dimensions distinguished by Hofstede (1991) as follows:

- Power distance focuses on equality and inequality between people in terms of power and wealth.

- Individualism is a way of life that is self-oriented. People in individualist cultures are independent. In contrast, collectivism is based on the group.

- Masculinity and femininity refers to social reinforcement of gender roles. In cultures with high masculinity men are dominant, and both men and women are tough.

- Uncertainty avoidance concerns feelings of anxiety, ambiguity, and fear of unknown situations. High uncertainty avoidance cultures pay more attention to formal rules, laws and orders, and getting the right answers.

- Long term orientation entails a sense of continuity. Cultures with high long-term orientation pay more attention to the values of long-term commitments and respect for tradition. 
Hofstede developed the first four cultural dimensions by surveying the attitudes of IBM's employees in over 50 countries in the 1960s, and added the fifth after using a sample of students in 23 countries. However, it is possible that the behaviour and attitudes of IBM staff may not accurately represent the characteristics of the national culture in each country. Furthermore, Hofstede did not pay attention to the history, political systems, and values of each country, although cultural differences derive from factors including religion, philosophy, political systems and specific values (Atmiyanandana and Lowler, 2003).

According to Hofstede (1991), Thai culture has characteristics of high power distance, collectivism, low masculinity, high uncertainty avoidance, and short-term orientation. The score of each dimension can be seen in table 2.1. These dimensions are based on IBM employees who have a high education level (IBM, 2005). In contrast, the vast majority of Thais have a low level of education and mostly work in the agricultural and manufacturing sectors (National Economic and Social Development Board, 2005). In order to clearly consider Thai culture, the Thai values, history, political systems, and Buddhist philosophy are taken into account. Thai culture can be viewed as having three characteristics: hierarchical orientation, flexible orientation and interdependent orientation.

Table 2.1 National culture dimension scores of Geert Hofstede for Thailand in 1991 (Hofstede, 1991)

\begin{tabular}{|l|c|c|}
\hline \multicolumn{1}{|c|}{ Dimensions } & Score & Rank \\
\hline Power distance & 64 & $21 / 22$ \\
Individualism & 20 & $39 / 41$ \\
Masculinity & 34 & 44 \\
Uncertainty avoidance & 64 & 30 \\
Long term oriented & 56 & 8 \\
\hline
\end{tabular}

\section{Hierarchical orientation}

According to history and political systems, Thai culture has a hierarchical orientation. Thai society inherited a class system based on an absolute monarchy that ranked citizens by land ownership (Holme and Tangtongtavy, 1997), and the current 
constitutional monarchy is still an important reinforcing factor for the hierarchical orientation of Thai society. Today, status in Thai society is ranked by wealth, age, gender (Atmiyanandana and Lawler, 2003), and education level (Komin, 1990). Thais acquire higher education in order to improve their social status rather than to obtain knowledge (Komin, 1990). The hierarchical orientation of Thai culture can be seen from the values of ' $k r e n g$ jai' and 'on nom thom ton', Buddhist philosophy and the concept of karma.

Kreng jai (to be considerate) is a common interpersonal behaviour ideal of Thais. Generally, Thais feel reluctant to impose, or make other people feel uncomfortable, and they always take other people's feeling into account. The degree of kreng jai depends on the status and relationship between people (Komin, 1990). There should be more consideration or kreng jai towards people of higher rank and seniority (Holme and Tangtongtavy, 1997).

On nom thom ton (humility) is another value that indicates the hierarchical orientation of Thai culture. Thais always perceive their status as lower than others. Particularly, subordinates and juniors members accept that they have less power and knowledge than superiors and seniors (Dubey-Villinger, 2001).

Finally, Buddhist philosophy and the concept of karma also reinforce the hierarchical orientation of Thai culture. A belief in karma means that an individual's current status is determined by his or her previous lives. Dubey-Villinger (2001, p. 107) states that “karma is the view that everything is predetermined or destined and one's position in society is of a static nature. One is therefore born into a social class and remains in that class till death”. As a result, Thais accept the power of higher status people and superiors because of the belief that they must deserve it. Moreover, belief in karma can lead to low motivation to change the current situation. Thus, these values indicate that Thai culture is more concerned with high power distance.

\section{Flexible orientation}

According to the findings of Hofstede (1991), Thai culture is one of high uncertainty avoidance which pays more attention to formal rules, and law and order. In fact, Thai culture is flexible and situation oriented. This can be viewed in terms of the value of 
'mai pen rai' (does not matter or no worries). Atmiyanandana and Lawler (2003) define mai pen rai in the terms of uncertainty and conflict avoidance. Most Thais can accept difficult situations by saying mai pen rai. In fact, they worry anyway but they treat their difficulties as unavoidable. Komin (1990) mentions that Thai culture is situation-oriented rather than system- and law-oriented. Thais do not pay as much attention to principles and regulations as to the person and situation. Furthermore, mai pen rai represents the hierarchical orientation of Thai culture. In Thai society, lower status people and subordinates have to respect their seniors and superiors, and accept those people's opinions and ideas. Thais generally say mai pen rai even when they have reservations, especially with seniors and superiors. This context also relates to the value of kreng jai or consideration. On this basis, mai pen rai confirms that Thai cultural values are low uncertainty avoidance and feminine.

\section{Interdependent orientation}

According to Hofstede (1991), Thai culture is collectivist. Conversely, in this author's opinion, Thai culture is interdependent rather than collectivist or individualistic. Atmiyanandana and Lawler (2003) claim that Thai culture is vertically collectivist, which can also be defined as “... a sense of serving the in-group and sacrificing for the benefit of the in-group and doing one's duty. ...the vertical dimension accepts inequality and rank has its privileges” (Triandis, 1995; p.44). Thais are reluctant to put forward their own ideas and opinions to a large group, but are comfortable within an in-group that believes in the same values and has seniors and higher status people as its representatives (Komin, 1990). On this basis, with an in-group, Thais are more informal and less kreng jai, thus, more sharing of their opinions. Furthermore, Thais are comfortable within an in-group because of the value of 'ruk sa naa' (face saving). Thais try hard to avoid expressing opinions or taking risks and responsibilities that could lead them to lose face. Thais are comfortable within in-groups that have the same status, age, gender, and education levels and shared responsibilities (Holmes and Tangtongtavy, 1997). Significantly, the interdependent orientation of Thai culture is also reinforced by Buddhist beliefs because the vast majority of Thais are Buddhist. The Buddhist beliefs of compassion, sharing and non-attachment to material and oneself influence Thais to share, especially between people within the same communities (Boonmathaya, 2003). 
On this basis, Thai culture can be viewed as having an hierarchical, flexible and interdependent orientation which contributes to high power distance, low uncertainty avoidance and collectivism respectively. Although the Thai values of kreng jai and on nom thom ton may impede sharing with those outside the community, Thais tend to be associated with informality, collaboration and sharing culture through the values of mai pen rai, interdependence and compassion.

Thus, organisational culture in Thailand is based on Thai culture. Because CEs are community-oriented, they are likely to be more concerned with collaborative sharing because they focus on in-group and informal orientation. The next section will discuss the topic of culture in the organisational context and how it can encourage the knowledge management of organisations.

\subsubsection{Organisational culture}

Culture in an organisational context is the set of meaningful beliefs, values, and assumptions of people in an organisation which are interpreted and acquired through their experiences. It can guide their actions (Geertz, 1973) and behaviours (Schein, 1992) on the basis of what should or should not be done, and whether something would have positive or negative consequences for the organisation. Schein (1992, p.12) defines organisational culture as

\footnotetext{
"A pattern of shared basic assumptions that the group learned as it solved its problems of external adaptation and internal integration, that has worked well enough to be considered valid and, therefore, to be taught to new members as the correct way to perceive, think, and feel in relation to those problems.”
}

Schein (1992) mentions that organisational culture derives from the experiences of members, and new cultures that come with new members and staff. For young organisations, organisational culture relies heavily on founders' behaviour and assumptions. Generally, organisational culture is created by the interaction of organisation members and behaviour justification, which are dynamic processes that allow members to share their own cultures and learn from the organisational culture (Ott, 1989; Schein, 1992). It is clear that organisational culture is influenced by the 
national culture or society of which it is a part (Tyson, 1989) and individual members (Ott, 1989). This is because members come to an organisation with their own cultures (Smircich, 1985) and transmit their culture through social interaction. As a result, culture is unique for each organisation. To examine the differences in this study, organisational culture will be considered from two perspectives: sociology and organisational management.

First, in the field of sociology, Goffee and Jones (1999) examine organisational culture in terms of two aspects: sociability and solidarity. Sociability focuses on social interaction, and the levels of sincerity and friendliness among organisation members. High-sociability creates an informal environment which increases trust and freedom in sharing and expressing ideas. Solidarity, on the other hand, is concerned with shared objectives. High-solidarity focuses on the strategic level and performance which can encourage commitment and loyalty to organisations. Meanwhile, Gopalakrishnan and Santoro (2004) consider the involvement, responsibility and commitment of organisational members as a culture of sense of ownership.

On this basis, CEs tend to have an organisational culture of high sociability and high solidarity. This is because CEs are community-oriented, home-based, and focused on social capital-social connection and relations-so, organisational members have numerous opportunities for interaction with each other in a friendly environment. In particular, the Prae Pun CE in northeast Thailand has high sociability based on its Isan culture, which means people in the same village are united and help each other, because they have close relations and a belief that the same spirits take care of the community and its members (Nartsupa and Lertwicha, 1994). Furthermore, villagers build a relationship with others whom they feel they can get along with through the 'pook siew' ceremony or real friend declaration ceremony (Buasri, 1983). After Isan villagers become real friends, they will visit and help each other, and may work together (Kritsanaphathi, 1983).

In terms of solidarity, CEs are owned by members, thus they have high commitment and loyalty to the organisation and focus on its goals. High commitment can be viewed as a strong culture because it comprises goal achievement and the dedication of organisational members (Kotter and Heskett, 1992). A strong organisational 
culture can influences members to accept shared understanding, goals and values, and normative behaviour (Littrell and Dickson, 1997), and involve them in learning and sharing. Therefore, it can be argued that high-sociability can enhance sharing among organisation members, while high-solidarity can encourage them to learn and create knowledge for the organisation.

Second, from the perspective of organisational management, the organisational culture dimensions of Hofstede (1991) and Alvesson (1993) can be considered under three aspects: operations, control systems, and communication. The first dimension classifies the operational systems of organisations into two types: process-oriented and result-oriented (Hofstede (1991), while Alvesson (1993) considers them to be goal achievement dimensions, which can be either objective or subjective. The process-oriented and objective dimensions focus on routine patterns with structure and robust systems. On the other hand, the results-oriented and subjective dimensions are concerned with outcomes and the consciousness of organisation members. Because CEs are home-based, often handcraft, industries, their operational system is more likely to focus on result orientation rather than the process orientation. Furthermore, the high-solidarity of CEs would lead to result orientation in the organisation as well as internal control.

The internal control systems of organisations can be either loose or tight (Hofstede (1991), which Alvesson (1993) calls free will and determinist. Loose internal control systems and free will dimensions pay less attention to formality, whereas tight internal control systems and determinist dimensions are restrictive. As mentioned previously, CEs tend not to be formally established. Their business activities often take place within the community. Thus, their organisational culture in this dimension is more likely to comprise loose internal control.

Finally, regarding communication in organisations, Hofstede (1991) considers behaviour under two styles: open and closed systems. The open systems allow free communication among organisation members, whereas the communication environment in closed systems is restrictive. CEs have an open system, because members are self-employed and engaged in casual employment. They are members of the same communities and familiar with each other. As mentioned previously, CEs 
have high sociability—-friendliness and interaction-that supports open communication.

In brief, it is safe to assume that CEs have an organisational culture of high sociability, high solidarity, result orientation, loose internal control and open communication. It is arguable that, even in nations having high power distance, individualism and high uncertainty avoidance, the organisational culture of their CEs would be similar. This is because of the specific characteristics of CEs. Wong and Aspinwall (2004) and Rasheed (2005) consider that small enterprises have an organic culture which is concerned with flexible and minimal direction in work processes, task orientation, success recognition, and an open-space form of organisation (Reigle, 2001). It has been shown that organic culture can also be referred to as result-oriented, loose internal control, high sociability and high solidarity. On this basis, the CEs in Thailand are likely to be the same as others. As discussed in the previous section, Thai culture has a flexible and interdependent orientation that contributes to Thai CEs' loose internal control, open communication, high sociability and organic culture.

\subsubsection{Knowledge management-driven culture}

Many scholars state that organisational culture can influence the KM patterns of thinking, behaviours and actions of organisation members (De Long and Fahey, 2000; Gupta, Iyer and Aronson, 2000; McDermott, O’Dell, 2001; Sveiby and Simons, 2002; Ribiere and Sitar, 2003; Lemon and Sahota, 2005). Organisational cultures which can influence KM are considered sharing cultures (Smith and McKeen, 2005) and knowledge or learning cultures (Despres and Chauvel, 2000).

\section{Sharing cultures}

Sharing cultures can be considered from two perspectives: knowledge sharing and cognitive sharing. Knowledge sharing refers to the willingness of organisation members to provide knowledge to others and to also accept knowledge from them (Smith and McKeen, 2005). From the perspective of cognitive sharing, this is concerned with shared meaning and mental models which can help organisation members to understand organisational culture and adapt to the environment (Levine and Moreland, 1999). The cognitive sharing occurs through the socialisation and interaction of organisation members (Levine and Moreland, 1999; Olivera and 
Argote, 1999). Thus, sharing cultures play a vital role in KM by influencing members to learn from the values of the organisation. In other words, cognitive sharing allows organisation members to acquire theoretical and cultural knowledge. Cognitive sharing also influences organisational learning (see section 2.3.5). Meanwhile, knowledge sharing can support KM through members acquiring knowledge from each other, especially tacit knowledge. Both knowledge sharing and cognitive sharing cultures are involved with sociability, loose control, and open communication systems. Two studies (Moffett, McAdam and Parkinson, 2002; Handzic and Agahari, 2004) confirmed that a collaborative culture and communication are key factors in encouraging sharing in organisations. As a result, CEs, especially in Thailand, tend to have an organisational culture of sharing as a support factor for their KM because they have informal and collaborative orientations.

\section{Learning cultures}

Learning cultures relate to organisational learning (Despres and Chauvel, 2000), and shared assumptions which can guide organisation members to behave as proactive problem solvers and learners (Schein, 1992). It has already been mentioned that culture can affect learning. People in individualistic cultures are more likely to be concerned with learning how to learn, while people in collectivist cultures tend to learn how to do things (Hofstede, 1991). In this researcher's opinion, both patterns are important for the knowledge process. Learning how to learn can encourage knowledge creation and the sharing of declarative knowledge. Meanwhile, learning how to do can encourage procedural knowledge creation and knowledge utilisation. Furthermore, learning how to do involves the practical application of knowledge processes. Particularly, high solidarity and result-oriented cultures can encourage organisational members to learn organisational topics. This is because solidarity can influence the commitment and loyalty of organisation members towards organisational goals (Goffee and Jones, 1999). From a Thai culture perspective, hierarchy can also influence people in learning. Even though Komin (1990) believes that Thais focus on learning in order to climb up the social rank rather than to gain knowledge, Katetade (1997) reveals that Isan culture gives respect to people who are superior in knowledge. 
On this basis, KM-driven culture is based on each organisation's culture and the culture of its members. CEs, as discussed above, tend to have sharing cultures and learning cultures which focus on organisational learning. The organisational learning will be discussed in detail in the following section.

\subsubsection{Organisational management}

As mentioned previously, knowledge is the output of learning and experiencing, so learning and sharing processes are crucial factors for organisational knowledge management. Pemberton and Stonehouse (2000) contend that knowledge is created through reasoning and inference. This section will consider organisational management as a factor which can encourage organisational learning processes, sharing and collaboration. Those factors include organisational structures and control systems and reward systems. This section will first examine organisational learning processes, followed by the collaborative environment, and reward and incentive systems.

\subsubsection{Organisational learning}

Argyris and Schon (1996) define organisational learning as the learning that individual organisational members acquire to enhance the organisation's potential and abilities. Stata (1996) further states that organisational learning is based on the shared insights and knowledge of organisational members, as well as on the past knowledge and experience of organisations. Thus, it can be said that organisational learning is more than the sum of individual learning. Organisational learning is concerned with the learning processes which aim to achieve organisational competencies and abilities. As a result, it consists of two main processes: behavioural and learning processes.

First, the behavioural process is concerned with the mechanism of adjusting organisation members' attitudes, and enhancing their willingness and commitment to learn in order to achieve organisational goals. This context can be referred to as the behaviourist or stimulus response approach, which is concerned with learning in response to changing stimuli (Jackson, 1993). Thus, the behavioural process plays the role of encouraging organisation members to learn about topics of organisational interest and to share their experiences and knowledge with other members. On this basis, it is argued that behavioural process focuses on activities of organisational goal 
recognition and commitment. According to social capital theory, social connection and relations encourage organisational members to focus on organisational interests. As a result, socialisation is a crucial activity. Goffee and Jones (1999) state that socialising both inside and outside the work place can increase the level of solidarity in workplaces. As discussed in section 2.3.4.2, high solidarity can influence trust, willingness, and commitment to organisations.

Second, the learning process is part of a cycle to create core competencies based on organisational contexts and memory. The learning processes can refer to the the cognitive approach which focuses on reasoning and creating experience, experimentation, and activities and analysis (Jackson, 1993). A learning cycle can consist of many activities, including competency building for operational systems or problem solving. Organisational competency building consists of five processes: problem identification, knowledge acquisition or solution finding, testing and reflection (Handy, 1995), knowledge storing, and knowledge distribution (Lopez, Peon and Ordas, 2004), whereas problem solving comprises four activities: problem identification, possible solution finding, testing, and reflection (Handy, 1995). First, problem identification is the process of informing organisation members about relevant topics. Second, knowledge acquisition is the process of finding a solution. Third, testing and reflection are the process of evaluating knowledge. Fourth, knowledge storing is the process of organisational knowledge asset management. It is concerned with content management, storage, and retrieval technology. Finally, knowledge distribution is the process of knowledge exploitation for organisational operations. It can be observed that the learning process is a part of the knowledge process. In comparison, knowledge acquisition (or knowledge capture) relies on three main sources: organisational memory, members' experiences and knowledge, and external sources. Furthermore, knowledge storing can also be considered as codification and organisational memory, and knowledge distribution is a way of utilising it. Organisational memory can be stored in several forms and locations including individuals, documents, records, culture, transformations, structures and ecology, and can be retrieved with several mechanisms, both formal and informal (Walsh and Ungson, 1991; Nilakanta, Miller and Zhu, 2006). Therefore, conducting learning processes involves implementing $\mathrm{KM}$ in organisations. 
For small enterprises, the learning processes tend to focus on informal learning (Wong and Aspinwall, 2004). because of their less formal organisational culture and operational systems, and limited resources as discussed above. Thus, CEs have to perpetuate knowledge through learning-by-doing, learning by observation and learning from others. Moreover, the high sociability and solidarity and social capital of CEs are well suited to informal learning processes.

\subsubsection{Collaborative environment}

As discussed in previous sections, a collaborative environment is a prominent factor in supporting knowledge processes. Such an environment involves socialising and acculturating between organisation members. Sveiby and Simons (2002) state that a collaborative environment enhances trust and the willingness of organisation members to participate in knowledge sharing. In addition, Lopez, Peon and Ordas (2004) found that a collaborative culture influences organisational learning in Spanish organisations by enhancing the sharing, trust, and long-term outlook of members.

Collaboration is a form of management in which several parties with shared interests join together in order to solve problems that cannot be addressed by one party (Gray, 1998). He points out that collaboration involves the processes of joint decisionmaking and collective responsibility. The collaborative environment depends on many factors including an organisation's structure and size, internal environment, and culture. Smaller enterprises tend to have a more collaborative climate (Sveiby and Simons, 2002). This is because small enterprises have a less hierarchical structure which allows organisation members to communicate easily with each other. Furthermore, according to Hall (1982), a decentralised organisation enhances the collaborative environment. This is because decentralisation allows a greater number of people to participate in decision making. Another factor is physical space and design: an open office layout is a crucial factor in enhancing sharing and communication among staff, and formulating trust (Handzic and Agahari (2004). Similarly, the organic culture of small enterprises leads to a friendly and collaborative environment (Wong and Aspinwall, 2004). This is confirmed by Handzic and Agahari (2004) who say that empowerment and decentralised organisational systems can enhance knowledge creating and sharing. Driver (2002) also affirms that learning organisations normally have flat structures. 
On this basis, CEs are likely to have high levels of collaboration within their organisations. They are often small in size and have casual operation systems with minimal structure. Moreover, CEs are home-based and community-oriented systems, so they would often have the simple style of office and working places mentioned in the section 2.1. From the organisational culture perspective, CEs are referred to as having an organic culture (see section 2.3.4).

\subsubsection{Reward and incentive systems}

Many scholars affirm that reward and incentive systems can influence the motivation and willingness of organisation members to share knowledge (Gupta and Govindarajan, 2000a; Bartol and Srivastava, 2002; Hislop, 2005; Smith and McKeen, 2005). Reward systems can reduce the reluctance of organisation members to share (Bartol and Srivastava, 2002) and motivate target groups to accept knowledge from others and maintain their knowledge stock (Gupta and Govindarajan, 2000b). Reward and incentive systems to encourage organisational members to participate in KM can be both extrinsic and intrinsic. Extrinsic reward and incentive systems are concerned with tangible values provided to motivate organisational members to work towards particular goals (Amabile, 1993; Bartol and Srivastava, 2002). Talisayon (2002), and Bartol and Srivastava (2002) mention that formal knowledge sharing through information technology, such as intranets and knowledge databases, can be consistently evaluated and rewarded with extrinsic reward systems. However, implementing reward and incentive systems for knowledge sharing can be difficult in practice, especially for informal knowledge sharing.

Conversely, intrinsic reward and incentive systems rely on the recognition and appreciation of groups. Intrinsic reward and incentive systems are intangible values that motivate people to work in order to fulfil their satisfaction and interests (Amabile, 1993). Wiig (1994) notes that the culture itself can be an incentive for the competency development of organisations. Intrinsic reward and incentive systems are more suitable for informal knowledge sharing (Bartol and Srivastava, 2002), in organisations with a result-oriented, loose internal control, and open communication culture concerned with performance outcomes and informal practices. These reward systems are therefore particularly suitable for small enterprises, such as CEs. This is because small scale businesses cannot afford to spend money on extrinsic incentive 
systems (Wong and Aspinwall, 2004). Furthermore, high solidarity organisational culture of CEs can enhance the willingness of the enterprise members to engage in sharing and learning. Wong and Aspinwall (2004), and Rasheed (2005) also find that small enterprises' organic cultures foster knowledge sharing and help members to easily understand their enterprise's achievements.

Many researchers argue that extrinsic reward and incentive systems can reduce the intrinsic motivation and willingness of organisational members to share their knowledge, because intrinsic motivation is derived from the enjoyment of doing tasks and self-actualisation needs (Gupta and Govindarajan, 2000b; Bartol and Srivastava, 2002; Hislop, 2005; Smith and McKeen, 2005). Nevertheless, extrinsic incentive systems, especially monetary rewards, are also necessary for CEs. With regard to CEs that are established to solve poverty problems (see section 2.1), their members are poor and need the income. Thus, reward and incentive systems for CEs should be balanced between extrinsic and intrinsic reward systems. There is a possible mechanism call 'extrinsics in service of intrinsics' which is concerned with use of actual rewards to support one's sense of competency without undermining one's sense of self-determination that encourages intrinsic motivation. The rewards can be provided by way of facilitating competency improvement and idea creation (Amabile, 1993). Amabile asserts that the monetary reward itself does not undermine intrinsic motivation and creativity. This can be explained by the basic needs of Maslow (1987). Generally, not only do people's physiological needs and safety needs (security and stability) need to be met, but also their self-esteem and the esteem of others, and selfactualisation needs.

\subsubsection{Appropriate technologies for KM}

It is generally agreed that information and communication technology is a very small part of KM, simply enabling it to become more effective and more explicit. Most scholars see the role of ICT as supporting knowledge processes, especially knowledge sharing and codification by facilitating interaction between people and organising knowledge in electronic forms (Davenport and Prusak, 1998; Sena and Shani, 1999; Reid, 2003; Abu-Rashed, Bertaux and Okunoye, 2005). This section will consider ICTs which can support knowledge processes, learning processes and collaborative environments by focusing on appropriate technologies for CEs in developing 
countries which have limited size and capabilities for handling ICTs (Abu-Rashed, Bertaux and Okunoye, 2005). ICT features for KM are concerned with collaboration and content management (Wyllie, 1998; Moffett, McAdam and Parkinson, 2004), and business intelligence which is focused on knowledge discovery and intelligent support systems (Moffett, McAdam and Parkinson, 2004). This study will not include business intelligence technologies — such as data mining, expert systems, and intelligent support systems - because they require a high level of investment including technological, human and financial resources.

First, collaborative technology is an important tool for KM. This is because collaborative technology involves tools which allow people to interact and conduct social activities through technologies. Currently, some collaborative technology-e.g. e-mail—is commonly used and available in most organisations. Other examples of collaborative tools used for KM are groupware, conference systems, directories and yellow pages (Moffett, McAdam and Parkinson, 2004). Thus, collaborative technology plays a crucial role in enhancing a collaborative environment to encourage learning processes and knowledge sharing, especially knowledge acquisition and distribution.

Second, content management is focused on content creation and knowledge organising, and information architecture which provides structure to support users in finding and accessing information (Wurman, 1997). Content management for the knowledge of enterprises consists of four components that can support knowledge processes: content creation, content repository or storage, content organising, and content distribution through business functions (Nakano, 2002; Alsup and Strong, 2004). Technologies that can be applied to content management include document management systems and web technologies (Wyllie, 1998; Moffett, McAdam and Parkinson, 2004). Document management systems consist of document creation in electronic forms, organising by classifying, indexing and searching technologies, distribution, and revision, which can involve web technology or specific software (Bielawski and Boyle, 1997). Thus, document management systems can underpin the processes of knowledge capture and codification, knowledge sharing, and learning through knowledge distribution. 
Therefore, content management with document management systems could potentially be feasible for CEs. This is because the Internet and web technology are widely available. Litan (2005) observes that Internet technologies have been diffused throughout the developing countries much the same as other electronic technologies. In terms of collaborative tools, they are focused on common tools such as e-mail and directories or yellow pages which are derived from data based systems rather than groupware and conference systems that require higher technologies and skills. This issue will be discussed in detail in the next section. However, CEs normally depend on face-to-face communication rather than technologies. Thus, collaborative tools may not be necessary, but internet technology could be very useful for facilitating knowledge organisation through document management systems and as a channel for sharing knowledge with customers. Martin and Matlay (2003) find that Internet technology in the form of organisation web sites can support small firms in acquiring knowledge from customers and fostering employees' learning. Given the limited resources and skills of employees, KM for CEs is constrained by a low level of ICTs based on off-the-shelf software and internet technology (Sparrow, 2001). As a result, ICTs are mainly used for knowledge organising and codification, and knowledge sharing between CEs and their customers, rather than for communication between CE members.

In Thailand, where IT 2000 is recognised as the first IT policy, ICT infrastructure has been developed throughout the country (Koanantakool, 2003). Public telephone services are available in almost every village in rural areas but only 30.6 per cent of the population use mobile phones, with most living in the central part of Thailand. In terms of Internet usage, in the same year, Internet users were calculated as comprising only 6 per cent of the population and most of them were in Bangkok (NECTEC, 2003), so this means few rural people could access the Internet. Gray, Kelly and Minges (2002) find that Internet users in rural areas made up only 9 per cent of Thai Internet users in 2001. This is because most rural people cannot afford the service and it is not a priority. Most rural families have trouble meeting daily expenses and have limited ICT skills (Koanantakool, 2003). As a result, they cannot get any advantage from low-cost PC and low-cost Internet policies. In terms of public Internet service, community telecentres (public ICT services at the community level) are only at the 
stage of pilot projects in four provinces (Koanantakool, 2003). Thus, it seems that so far ICTs appear mainly in policy or as pilot projects in rural areas.

Arguably, most CEs' members in rural Thailand do not rely on ICT for communication with each other and knowledge sharing. ICTs would be used only at office level by administration staff. ICTs would be communication channels between administrators, and supporting agencies and customers rather than among members. In terms of knowledge organising, CEs may have low specification of PCs with common software such as word processing and spreadsheet.

\subsection{Knowledge management in Thailand}

\subsubsection{Knowledge management models for Thai society}

In 2003, the Knowledge Management Institute (KMI) in Thailand designed a knowledge management model for Thai society, in particular for people and the public sector. The model is focused on problem solving and knowledge creation through learning-by-doing. Interestingly, the KM model of KMI is based on Buddhist principles. In terms of problem solving, the KMI designed the model based on '4Ariyasaj' or four noble truths which is concerned with causes and effects of distress, and solutions. 4-Ariyasaj consists of four truths: Dukkha or the nature truth of suffering, Samudaya or cause of suffering, Nirodha or the cessation of suffering, and Mugga or the way leading to the cessation of suffering (solution). First, the problem component is represented by Dukkha which comprises causes and solutions. Second, KMI refers to knowledge sources as Samudaya or causes of suffering because people who have no knowledge are considered to be suffering according to Buddhist principles (LearnTripitaka.com, 2005). Thus, the strategies for problem solving can be defined as Nirodha or the cessation of suffering. Finally, knowledge assets are equated with Mugga or solutions.

In terms of knowledge creation, KMI designed the learning process based on two Buddhist principles: '4- iddhipatihariya' or power of transformation, and 'Prom Vihan' which is the Dhamma principle for daily activities. 4- iddhipatihariya is concerned with successful strategies: willingness, effort, attention and concentration, and follows on the success of cause and effect (Buddhadasa.com, 2008). Prom Vihan involves mercy and admiration. It can be assumed that KMI suggests KM solutions 
for relieving suffering on the basis of searching for knowledge and sharing between knowledgeable and unknowledgeable people as givers and receivers.

The KM model consists of six components: people, knowledge, knowledge asset, KM processes, problems, and tools (KMI, 2005a). Figure 2.2 shows all components of the the KM model proposed by KMI.

- People can be categorised into two groups: organisation members and external experts. External experts who are involved in $\mathrm{KM}$ for people and the public sector can be categorised into three groups: local experts, scholars, and people from support agencies, such as governmental staff and NGO workers.

- Knowledge sources in the KM model can be divided into two sources: external and internal sources. Most knowledge from external sources is explicit knowledge from external experts, although knowledge from local experts is mainly tacit knowledge. Conversely, most internal knowledge is also tacit knowledge: local wisdom or indigenous knowledge, culture, beliefs, and experiences.

- The knowledge asset is a store for new knowledge and lessons learned from activities.

- There are four knowledge processes: capturing, sharing, creating, and using. Knowledge creation and sharing are focused on learning processes which consist of three steps: learning before an event, learning during an event, and learning after an event (KMI, 2005c). It can be seen that this KM model lacks the processes of knowledge verification and knowledge transfer. As discussed in section 2.3.3, it is necessary for an organisation to test and review its knowledge in order to verify its appropriateness to the current situation. Meanwhile, knowledge transfer is a vital process for organisational competency development. As discussed in section 2.3.5.1, past organisational knowledge is a factor for learning, which is a process of organisational competency development.

- Problems and objectives are key components of the model. These components determine the sources of knowledge to capture, and the knowledge to use, share and create. 


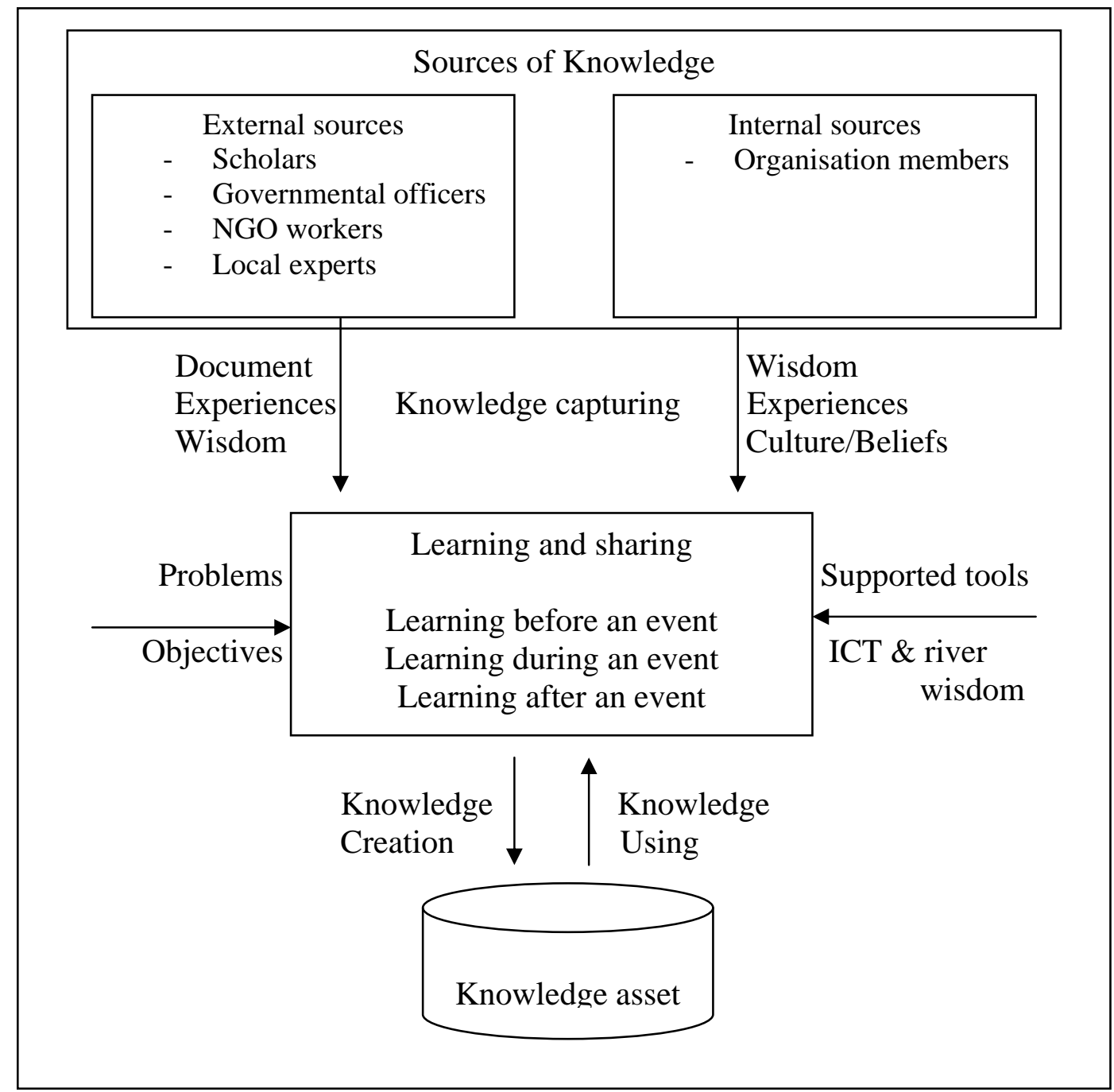

Figure 2.2 The first KM model of KMI Thailand adapted from KMI data(2005a, 2005b)

- Tools. KMI has provided two tools: ICTs and 'Thran Pannya'. First, KMI (2005a) mentioned that possible ICTs for this model are intranets, websites, web discussion boards, and communication software. Another tool is Thran Pannya (river wisdom) which has been used to facilitate knowledge sharing. Thran Pannya is derived from the KM model of British Petroleum, designed by Geoff Parcell. It consists of five elements: a self-assessment table, river diagram, stair diagram, knowledge asset, and sharing space (KMI, 2005b).

1) The self-assessment table is used for core competency evaluation. The core competency can be divided into three main competencies: competency in action, 
in understanding, and in values and norms. The core competencies are given one of five levels: beginning (level 1), moderate (level 2), good (level 3), very good (level 4), and excellent (level 5). Figure 2.3 gives an example of a self-assessment table.

2) The river diagram is used to present the core competencies of groups or members which are gathered from the self-assessment table. The diagram is used to compare core competencies of each group and to indicate the sources of each competency in a network or organisation. As a result, river diagrams can be used to facilitate knowledge sharing. Figure 2.4 gives an example of a river diagram.

3) The stair diagram presents the current level of each core competency and the amount of effort that each group or group member needs to make in order to achieve the expected level. The groups or group members that are located at the top of the diagram are competent, so they are knowledge providers in terms of knowledge sharing. Conversely, groups or group members that are located at the lower-right of the diagram are lacking those skills. They would be the receivers in the knowledge sharing process. Figure 2.5 gives an example of a stair diagram.

4) A knowledge asset is created from knowledge sharing and learning processes. The asset focuses on contexts and explicit detail, such as audio and visual samples.

5) Sharing spaces focus on available sharing channels. There can be both face-to-face and virtual sharing spaces where knowledge providers and knowledge receivers can share their knowledge. 


\begin{tabular}{|l|l|c|c|c|c|}
\hline \multirow{2}{*}{$\begin{array}{c}\text { Core competency } \\
\text { issues }\end{array}$} & \multicolumn{5}{c|}{ Level of competencies } \\
\cline { 2 - 6 } & $\begin{array}{c}1 \\
\text { Beginning }\end{array}$ & $\begin{array}{c}2 \\
\text { Moderate }\end{array}$ & $\begin{array}{c}3 \\
\text { Good }\end{array}$ & $\begin{array}{c}4 \\
\text { Very good }\end{array}$ & $\begin{array}{c}5 \\
\text { Excellent }\end{array}$ \\
\hline Core competency 1 & & & & & \\
\hline Core competency 2 & & & & & \\
\hline Core competency 3 & & & & & \\
\hline Core competency 4 & & & & & \\
\hline Core competency 5 & & & & & \\
\hline Core competency 6 & & & & & \\
\hline Core competency 7 & & & & & \\
\hline Core competency 8 & & & & & \\
\hline Core competency 9 & & & & & \\
\hline Core competency 10 & & & & & \\
\hline Core competency 11 & & & & & \\
\hline Core competence 12 & & & & & \\
\hline
\end{tabular}

Core competencies evaluation

Core competencies are divided into many issues according to the KM vision.

There are five core competency levels. Each group or group member has to evaluate themselves and tick one of each core competency level rows.

Figure 2.3 Self-assessment table used to evaluate core competencies of groups or group members (KMI, 2005b) 


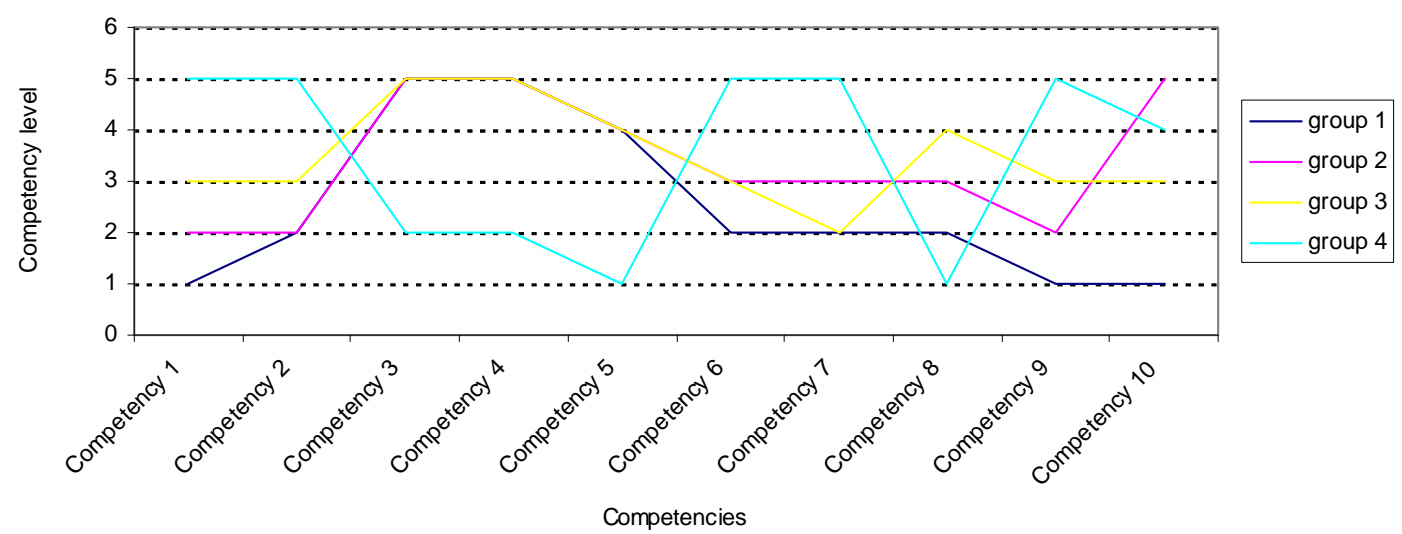

River diagram

The diagram represents levels of core competencies of each group. The graph shows sources of each competence. The groups that are located on the top of the graph for such competences are knowledge providers for knowledge sharing and vice versa.

Figure 2.4 River diagram (KMI, 2005b) 
Competency 1

\begin{tabular}{|c|c|c|c|}
\hline Group & $\begin{array}{c}\text { Current } \\
\text { competency } \\
\text { level }\end{array}$ & $\begin{array}{c}\text { Target } \\
\text { competency level }\end{array}$ & $\begin{array}{c}\text { Amount of } \\
\text { effort }\end{array}$ \\
\hline 1 & 1 & 4 & 3 \\
\hline 2 & 2 & 4 & 2 \\
\hline 3 & 3 & 5 & 2 \\
\hline 4 & 5 & 5 & 0 \\
\hline
\end{tabular}

Stair diagram of core competency 1

Current core competency level

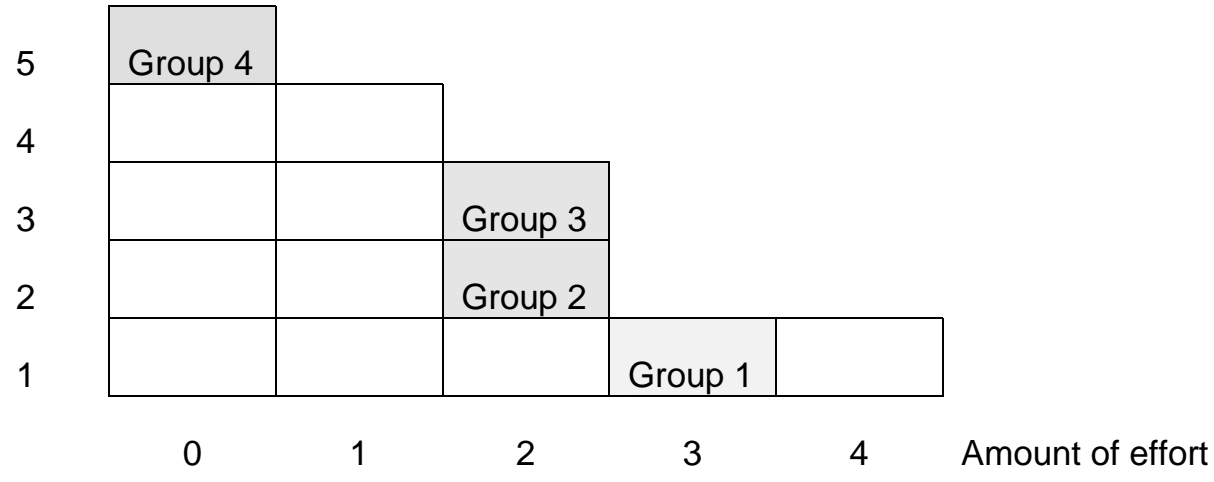

Stairs diagram

Group 1: Need level 3 amount of effort in order to have core competency 1 at level 4. Group 2: Need level 2 amount of effort in order to have core competency 1 at level 4 Group 3: Need level 2 amount of effort in order to have core competency 1 at level 5 Group 4: No effort needed because core competency 1 was already at level 5. Group 4 would be a knowledge provider, while groups 1, 2 and 3 would be knowledge receivers in knowledge sharing process.

Figure 2.5 A stair diagram drawn from levels of current core competency and target core competency (KMI, 2005b). 
In 2004, KMI designed the “carp model” as an alternative KM model for Thai society. The carp model is an extended KM model which presents a strategy for large organisations or networks that have many divisions or group members. The carp model consists of a large main carp and many small carps in the form of a mobile. Each carp consists of three components: KM vision, knowledge sharing, and a knowledge asset (see figure 2.6). The big carp represents an organisational KM model, whereas the small carps represent each division's KM model. The KM model of each division has the same vision and objectives as the main model but has its own strategies in knowledge sharing and a knowledge asset. The carp model continually focuses on problem solving and knowledge sharing to create a knowledge asset.

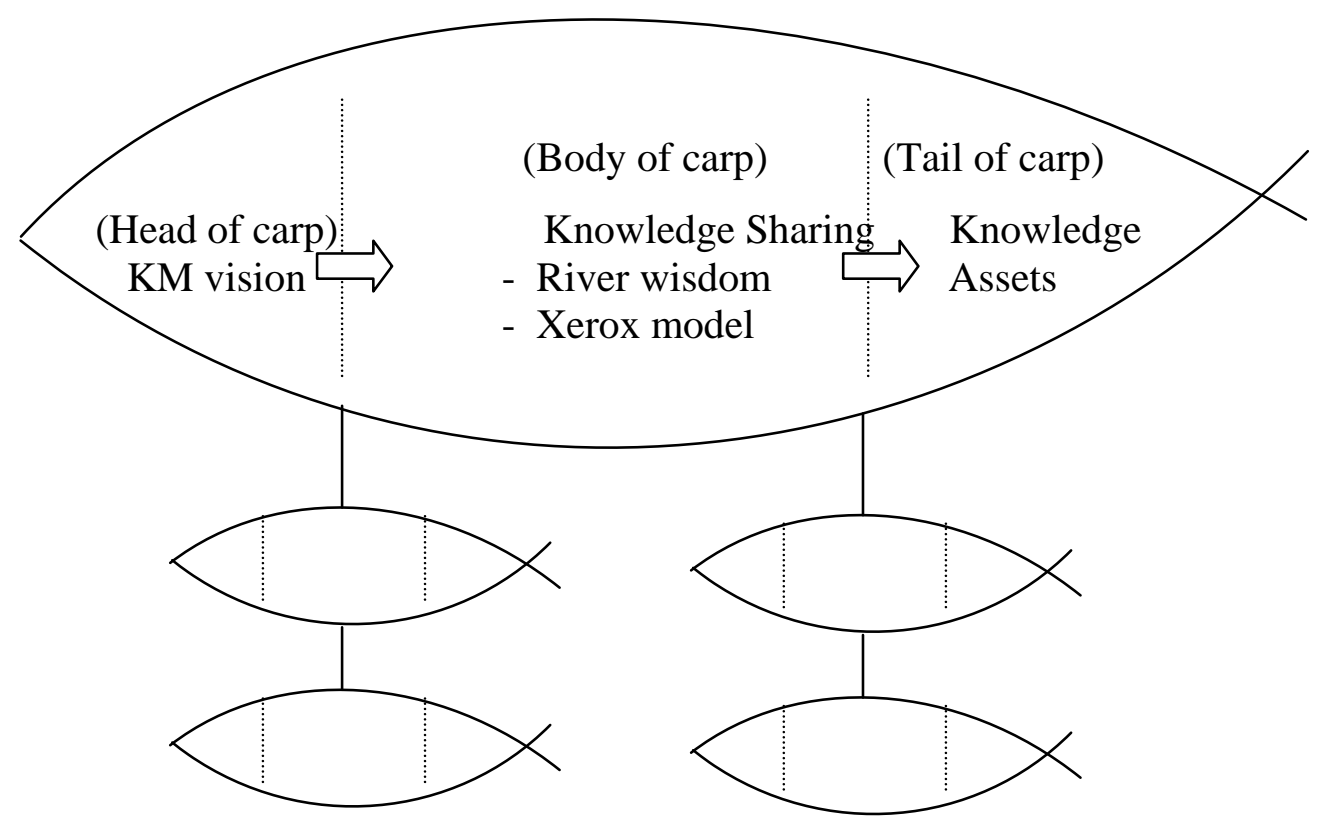

Figure 2.6 Carp model, adapted from KMI data (2005a, 2005b).

Compared with the 2003 KMI model, the carp model focuses on organisational contexts through its knowledge sharing tools: Thran Pannya and the Xerox model. In the carp model, the self-assessment table of Thran Pannya is used to reflect the KM vision, the river diagram is used as a tool for knowledge sharing, and the stair diagram is used to reflect the knowledge asset of the KM model (see figure 2.7). It can be seen from figures 2.3-2.5 that Thran Pannya is quite a complex tool for people who have few skills in documentation. As mentioned previously, KMI has designed KM for 
people and public sector. The vast majority of Thais are rural people who are not familiar with documentation. As a result, this model is suitable for official use and other parties would be required to facilitate its use for grass root communities.

Alternatively, the Xerox model which takes a practical approach has been applied as a knowledge sharing tool to support the carp model (KMI, 2005b). The Xerox model comprises six components: transition and behaviour management, communication, processes and tools, training and learning, measurements, and recognition and rewards. The 'Shivalai' project which is a community learning centre in Satuk district, Burirum province, has applied the carp model using the Xerox model as a knowledge sharing tool for KM (see figure 2.8). Thai Rural Net (TRN) (2005) states that the carp model along with the Xerox model can support the KM vision of the Shivalai project in community problem solving and community self-development.

It is clear that the carp model with Thran Pannya is more suitable for organisations that have documentation skills rather than rural community organisations such as CEs which lack those skills. Thran Pannya relies on paperwork, data analysis, and graph interpretation, which are less applicable to CEs, so the carp model with the Xerox model is a more practical alternative. This is because the Xerox model provides clear direction for knowledge sharing. According to KMI (2005a), Thran Pannya has been used by the public sectors, such as the hospital KM network in the lower north region which has extensive documentation and data analysis skills, whereas the Xerox model has been applied by rural community organisations which are activity-based. 


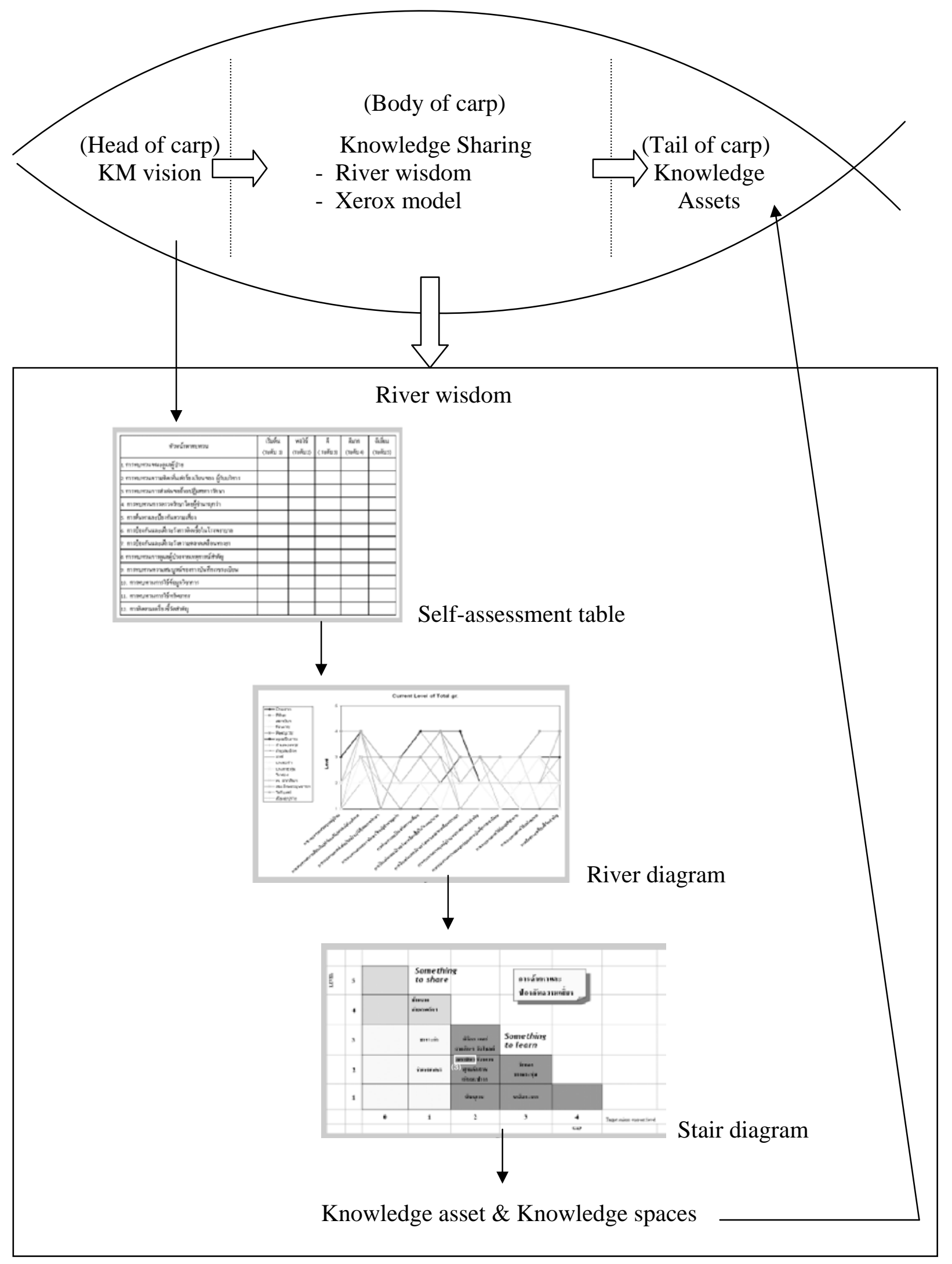

Figure 2.7 Carp model with “Thran Pannya” (River wisdom) as a knowledge sharing Tool adapted from KMI data (2005a, 2005b). 


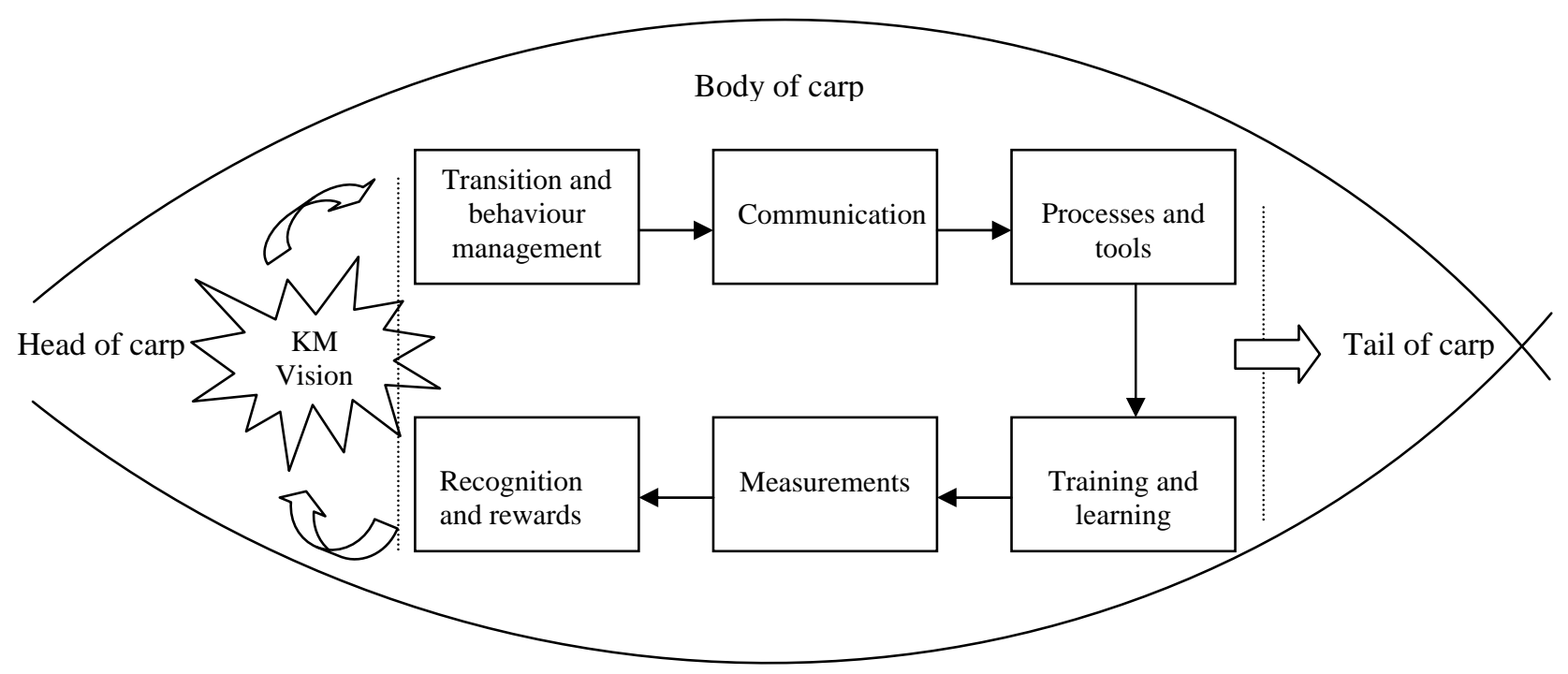

Figure 2.8 Carp model with Xerox model as a knowledge sharing tool adapted from KMI data (2005a, 2005b).

\subsubsection{Basic KM models of KM in Thailand}

As mentioned in section 2.4.2, KMI has adopted two KM models for Thai society based on two original KM models: BP's model and the Xerox model.

\subsubsection{BP’s KM model}

BP's KM is based on its business activities and focuses on two main components: the learning cycle and knowledge assets (Collison and Parcell, 2004; SAIC, 2005). KM activities in BP's model consist of knowledge capturing, learning processes, knowledge validating and distilling, knowledge transferring, and knowledge applying (see figure 2.9). First, knowledge capturing is the process of capturing know-how from staff to generate corporate knowledge or knowledge assets, which can be reused effectively. Second, the learning process is divided into three steps: before, during, and after an activity. Third, knowledge validating and distilling are the processes of approving a knowledge asset. Next, transferring is the process of distributing knowledge assets to staff through a training process in order to ensure that staff exploit the knowledge. Finally, knowledge applying is the process of adapting and adopting knowledge for business activities (Collison and Parcell, 2004). 


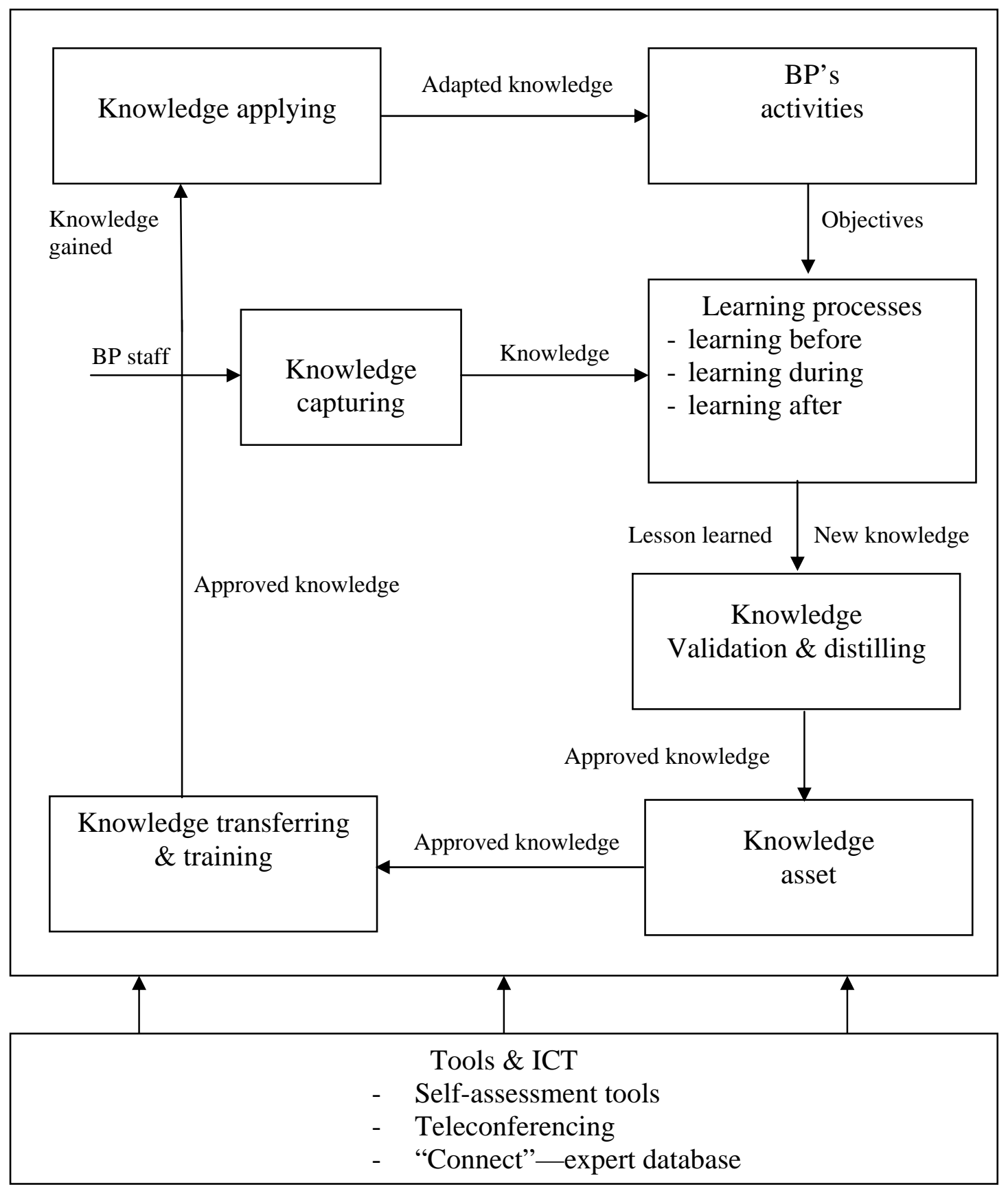

Figure 2.9 BP’s Knowledge Management model, adapted from Collison and Parcell (2004)

Furthermore, main strategies in the knowledge capturing and the learning process of BP's KM are communities of practice (CoP) and peer assistance (Collison and Parcell, 2004; SAIC, 2005). BP's KM is focused on an informal network of sharing and learning by staff in similar roles and contexts (Dixon, 1999; Lesser and Prusak, 
1999; Kankanhalli et al., 2003). Peer assistance can encourage staff to request help from colleagues at the same level to work in the team (Dixon, 1999). Kankanhalli and colleagues (2003) mention that BP has used an expertise profile database to support staff in finding peer assistance.

In fact, BP has created a peer process which comprises three strategies: peer group sharing, peer review and peer assistance. Peer group sharing means sharing knowhow between businesses, whereas peer review is the process of knowledge inspection by professionals from similar business activities, and the peer assistance is the process of requesting specialist help (Collison and Parcell, 2004). Thus, the peer processes involve knowledge capture, knowledge validation, and knowledge transfer.

BP uses ICTs to facilitate its KM in two main ways. Firstly, it uses ICTs to facilitate knowledge sharing worldwide through video-conferencing (Collison and Parcell, 2004). Secondly, BP uses ICTs to create "Connect"- expert directories (Kankanhalli et al., 2003). It is clear that BP's KM model is suitable for organisations with similar business networks. Each business can share its experiences with others in the network. Like BP, rural CEs in Thailand tend to manage their businesses as a network of group activities. Nevertheless, BP's KM tools and techniques for knowledge sharing are far from a rural community's capabilities and skills.

\subsubsection{Xerox’s KM model}

$\mathrm{KM}$ at Xerox is focused on knowledge sharing, based on ICTs and incentive systems. Xerox has two sharing tools: Eureka and DocuShare. Eureka is a knowledge repository system which has been implemented in 71 countries and contains approximately 50,000 technical tips (Roberts-Witt, 2005). Eureka allows service staff to access and share their repair tips with each other whenever and wherever they prefer through collaborative tools (Hickins, 1999; Holtshouse, 1999; Roberts-Witt, 2005). Knowledge that is submitted to Eureka is reviewed and validated by experts before it is made available (Holtshouse, 1999; Kankanhalli et al., 2003). As regards incentives, Xerox provides reward systems to encourage staff to engage in collaboration and sharing. The Xerox knowledge sharing model is concerned with four components: behaviour, technology and tools, processes, and people (see figure 2.10). Staff behaviour entails encouraging staff to willingly share knowledge via the 
Eureka knowledge asset. Technology and tools are focused on IT for collaboration and sharing. There are three main processes: knowledge sharing, knowledge validation, and knowledge codification. People can be divided into three main groups: service team members, domain experts, and executives. Service team members are involved in knowledge sharing and using, while domain experts contribute through knowledge validation, and executives try to adjust staff behaviour.

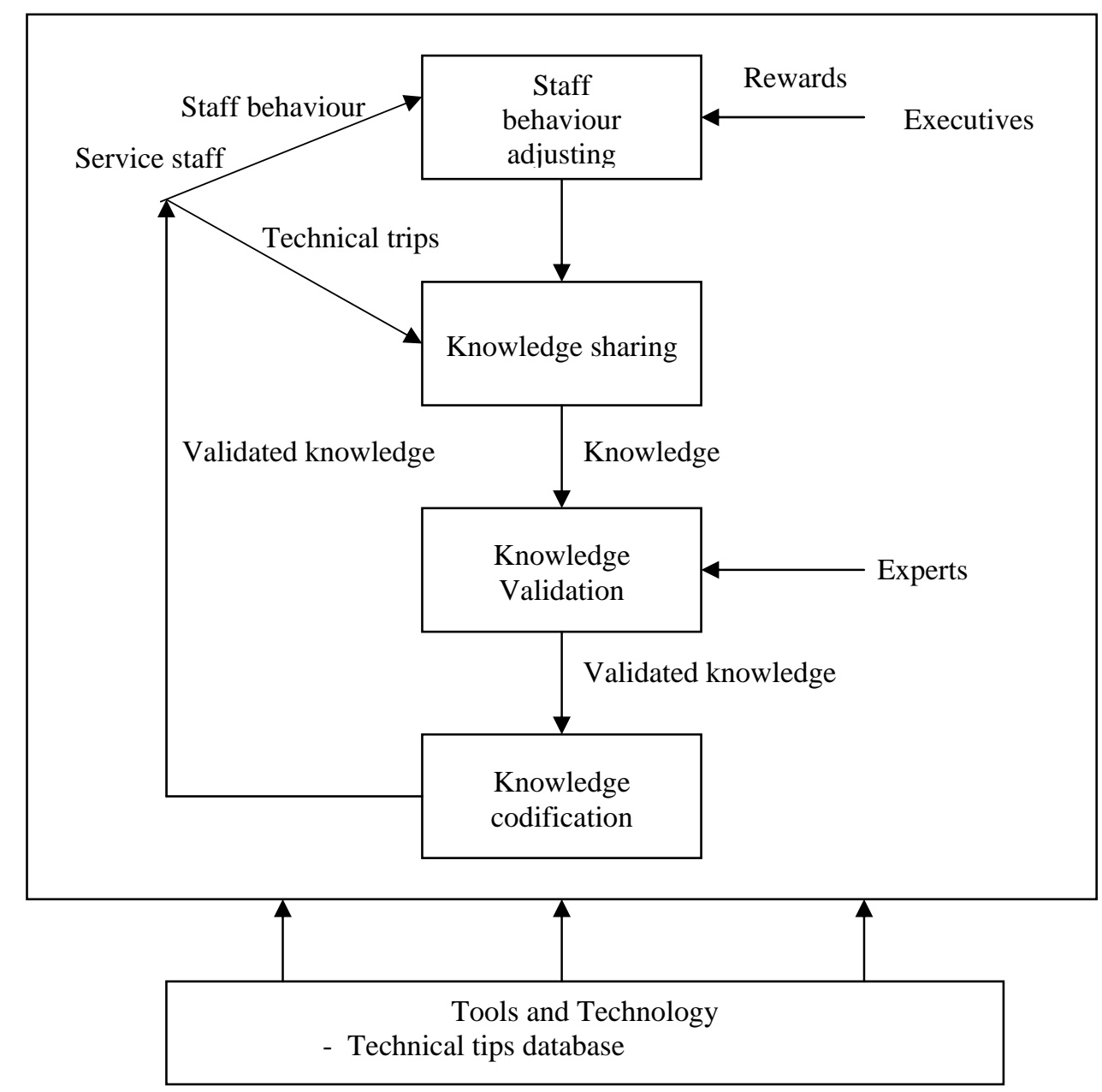

Figure 2.10 Eureka model: knowledge sharing model for service staff of Xerox, adapted from Holtshouse (1999), Kankanhalli et al. (2003) and RobertsWitt (2005).

DocuShare is an intranet-based document repository which was developed to support production development teams in sharing their information and knowledge. DocuShare was designed to encourage scientists who do not like collaboration and socialising to share and access knowledge and experiences from the same context 
(Hickins, 1999; Holtshouse, 1999). It can be seen that Eureka collects tacit knowledge from service workers, while DocuShare gathers explicit knowledge from research and development teams.

Both the BP and Xerox KM models focus on sharing and capturing tacit knowledge and practical methods. BP's KM model is concerned with learning processes to create knowledge and applying knowledge for activities, while Xerox’s KM model (Eureka) is concerned with knowledge capture and distribution to staff. Furthermore, Xerox has designed its model on the basis of staff behaviour and its context.

Knowledge asset creation is considered a main objective of KM in these two organisations. Although the Xerox model does not mention knowledge assets, it does include the process of knowledge codification to create a technical tips database which can be considered a knowledge asset. Moreover, both organisations are concerned with knowledge validation in the knowledge creation process. BP has validates new knowledge through its peer review processes, while Xerox uses domain experts.

Sharing and transferring are significant $\mathrm{KM}$ processes in both organisations. Knowledge sharing at BP focuses on learning processes, while Xerox focuses on knowledge exchange among technical staff. Thus, BP's knowledge sharing can influence knowledge creating more than the Xerox model. Furthermore, BP has formal knowledge transfer through training to ensure that organisational knowledge is transferred to staff, especially those whose knowledge is limited. Finally, both organisations use ICTs to support knowledge sharing, transferring, and knowledge asset management.

\subsection{Knowledge management for community enterprises}

Given the characteristics and limits of CEs, KM for CEs tends to focus on the personalisation approach with informal processes and a low level of ICT. This is because small enterprises have small scale organisations and flat organisational structures, informal operational systems (Wong and Aspinwall, 2004; Sparrow, 2005), organic organisational culture (Wong and Aspinwall, 2004; Rasheed, 2005), local resources and social capital (Sparrow, 2001), and a low level of technology (Sparrow, 
2001; Martin and Matlay, 2003). Therefore, KM for CEs is not merely a smaller scale model of KM for large enterprises, as noted by Hylton (2002). Most KM for large enterprises focuses on ICTs, whereas CEs have limited funding for ICTs or staff skilful in computer literacy. Sparrow (2001) mentions that small firms focus on KM principles and knowledge processes that use intellectual and social capital. As a result, this author argues that KM for CEs is likely to be based on their work processes and interaction between relevant agencies.

Compared with BP and Xerox, CEs have informal operation systems, high sociability and solidarity, human capital and social capital, but low levels of ICTs, qualified employees and finance. Moreover, CEs' cultures tend towards more flexible and interdependent orientation. This is because CEs have a community orientation which focuses on collaboration and an informal in-group environment. Thus, the KM processes of CEs are likely to be different, so it is important to ensure that a model matches their special characteristics and needs. Each component of KM will be considered as follows. First, given that CEs tend to be home-based and informal, knowledge processes should be focused on informal and problem solving processes to acquire knowledge for activities. However, some formal knowledge processes, such as knowledge capture through training programmes, would also be suitable for CEs. As mentioned above, CEs lack experts. Thus, training programmes would provide knowledge from external sources for staff, but the programmes would need to incorporate informal processes. Second, given the interdependent culture, knowledge processes for CEs are likely to focus on collaboration, such as group work, discussion and CoPs. Third, due to CEs' focus on local knowledge and social capital, their knowledge processes should focus on socialisation and interaction. Knowledge sharing would involve informal processes rather than using technology, which is more likely to be applied only for knowledge codification. CE members mainly communicate face-to-face, therefore a KM model for CEs could be limited to simple technologies and require administration staff or volunteers to operate the system rather than direct` use by the CE members.

\subsection{The theoretical framework}

According to the literature review, CEs are classified as MSEs and part of the informal sector, whose main characteristics are flat structure and informal operation 
systems. Their businesses tend to be based on local knowledge, skills and indigenous knowledge from enterprise members and local experts, and limited resources including raw materials, human resources, financial resources and technologies. However, CEs are different from general MSEs in their objectives and ownership. CEs are established for community development and owned by community members collectively rather than individual owners. Thus, CEs have different characteristics and organisational cultures. CEs exhibit high sociability, solidarity and social relationships which are considered their social capital. CEs in developing countries tend to exhibit a high power distance and hierarchical orientation that influence members' reluctance in expressing ideas. Most CEs are located in the rural areas of developing countries, and have flexibility and interdependence, which suggests the organisational culture of CEs can be described as organic. Typically the organisational management and KM of CEs are based on informal processes to match their cultures and characteristics.

To extend understanding about CEs and their KM, this research seeks to explore the characteristics, organisational culture, and current KM practice of a CE, and to create a KM model in order to answer the research questions. As shown from the literature review, there is no conceptual model for proposing a KM model for CEs. Thus, a theoretical framework is developed to guide and direct the proposed research. The theoretical framework, based on the literature review, covers the two key issues of this study: CEs and KM for CEs. The literature review indicated that the KM model for CEs should focus on four components: KM processes, organisational management, organisational culture, and appropriate ICT, as shown in figure 2.11.

In order to understand CEs, this study explores their characteristics in five aspects: objectives, owners, management and operational systems, organisational culture, and resources. To explore CEs' KM practice, this study focuses on four components: knowledge types, knowledge processes, organisational management, and appropriate technology. It can be seen from figure 2.11 that KM processes play a significant part in creating an organisational knowledge asset and the other components support the KM processes. First, CEs' characteristics-resources, management and operation systems, and organisational culture-play a crucial role to determine the approach and methods of knowledge processes. Second, knowledge types are another factor for 
determining the knowledge processes, with, for instance, tacit knowledge associated with interaction and dynamic processes. Third, organisational management provides direction and a suitable environment for KM, focused on organisational learning, creating a collaborative environment, and reward systems. Finally, given the characteristics and culture of CEs, ICTs play more of a role in knowledge capturing and organising rather than knowledge creation, sharing, and utilising. Furthermore, ICTs for CEs' KM tend to be common technologies including off-the-shelf software and internet technologies.

Therefore, a KM model for CEs will be created on the basis of the characteristics of a CE case study, the Prae Pun handweaving enterprise in northwestern Thailand, its current practice and possible adoption of modern KM or knowledge processes. The framework plays a key role in the design of the study and monitoring its progress. Chapters 3 and 4 describe the research methodology, research design and research procedures. Chapter 5 presents stories from the field work in order to provide a clear picture and context of the research site. Chapter 6 describes the characteristics of the CE through stories from the respondents and observations of the researcher. Chapter 7 discusses the current KM practice of the CE, meanwhile, the KM model for CEs is described in chapter 8 based on the evidence from the case study, and theoretical and modern KM processes that are appropriate for CEs' situations. The exploration and the KM model are designed to answer the two research questions:

1. How does the community enterprise currently manage its knowledge?

2. Can theoretical and modern KM concepts be applied in an appropriate manner to strengthen traditional KM practice of the community enterprise? 


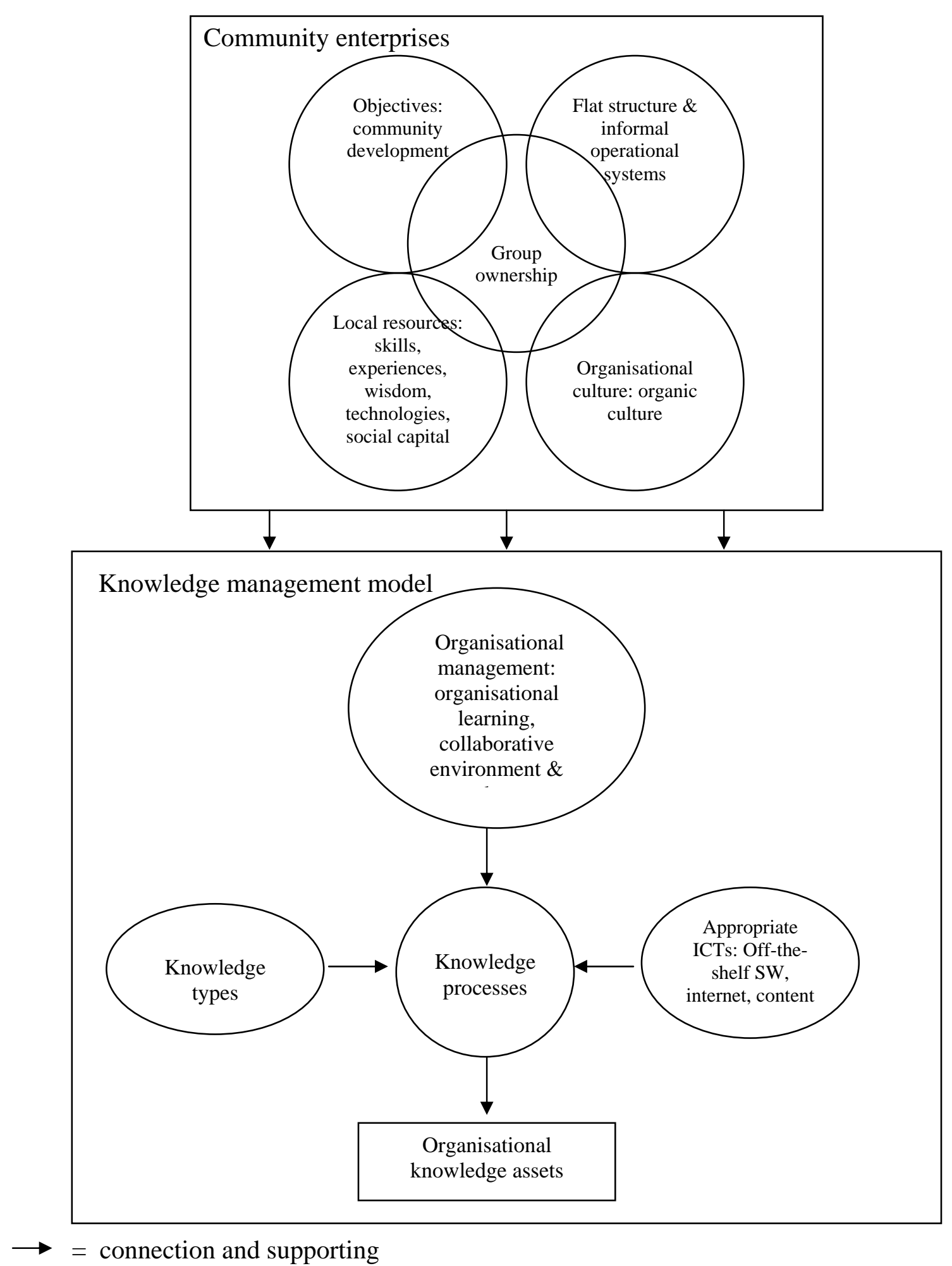

Figure 2.11 A theoretical framework for generating a KM model for community enterprises 


\section{Chapter 3}

\section{Research Methodology}

This chapter describes the research methodology and is divided into two parts. The first part focuses on paradigms and methodology, especially in knowledge management research. The second part describes the research method and design of this study.

\subsection{Research Paradigm}

This research is based on an interpretivist paradigm which is is concerned with understanding human behaviour and action (Bryman, 2004). It allows researchers to understand the world of meaning by interpreting, clarifying and constructing meaning from social phenomena, and focuses on the subjective experiences of people and the uniqueness of human enquiry rather than measuring phenomena and events. Furthermore, the interpretivist paradigm allows researchers to present social phenomena and subjective experiences scientifically with empirical methodology (Schwandt, 1994). As a result, an interpretivist paradigm fulfils the main objectives of this research: to explore the characteristics of a CE and its current KM practice in order to present a suitable KM model. In information systems (IS) development, developers have to acquire knowledge about an organisation in order to design an effective system for it (Hirschheim and Klein, 1989). Information systems (IS) research which aims to understand the context of IS production is based on interpretivism (Walsham, 1995). Meanwhile, IS-based KM research that uses the interpretive paradigm focuses on situations of organisations, roles of knowledge in organisations, roles of technology in supporting $\mathrm{KM}$, and organisational practices in organisational learning (Schultz and Leidner, 2002). Therefore, the interpretivist paradigm allows the researcher to understand a CE through its social phenomena and the personal experiences of individual members, and to use such understanding as requirements and constraints to create the CE's KM model.

\subsection{Methodological Approaches}

Given the main objectives and research paradigm of this research, this study takes a qualitative approach. Qualitative research derives from interpretive and 
phenomenological paradigms (Cassell and Symon, 1994). Qualitative research refers to a research paradigm that addresses questions of meaning, interpretation and socially constructed realities (Tashakkori and Teddlie, 2003). Another crucial reason for its use in this study is that qualitative research is focused on understanding real and complex phenomena (Cassell and Symon, 1994; Glesne, 1999, Bryman, 2004) through understanding individual behaviour (Cassell and Symon, 1994) and unique situations (Myers, 1997). Qualitative research is concerned with empiricist and exploratory research in social settings (Cassell and Symon, 1994; Glesne, 1999) and individual interpretation of researchers (Cassell and Symon, 1994; Glesne, 1999) in order to generate rather than test theory (Tashakkori and Teddlie, 2003).

From a practical point of view, qualitative researchers first seek to understand the social setting as it really is (Gubrium and Holstein, 1997). Second, qualitative researchers understand the social setting through naturalistic orientation methods (Gubrium and Holstein, 1997) with minimal contamination of events and specific instruments (Bryman, 2004). Third, qualitative research is concerned with subjectivity (Cassell and Symon, 1994; Gubrium and Holstein, 1997; Bryman, 2004), and gaining access to an insider view of studied societies (Guba and Lincoln, 1994). Finally, qualitative research is focused on sensitivity to the uniqueness of the setting. Given this, qualitative researchers understand social settings through specific environments (Bryman, 2004) and small samples (Willamson, 2006), and collect data with less structured methods that tend to be used in ethnographic approaches (Bryman, 2004).

However, qualitative research is criticised for providing subjective and narrowly applicable findings which are difficult to generalise (Cassell and Symon, 1994; Bryman, 2004) and lack of transparency (Bryman, 2004). In order to generalise findings, researchers have to provide rich insights from respondents and contextual information. Guba and Lincoln (1994) stated that rich insights into human behaviour, and individual and contextual information can enable generalisation with high theoretical rigour. As a result, this research employed narrative analysis with ethnographic techniques as data collection methods in order to minimise influence on the research site and gain the insights of respondents through the individual experiences of the CE's members. Those methods involved the researcher gaining rich data and in-depth understanding. In order to provide transparency, this study's 
research site and respondents were carefully chosen with purposive sampling which allowed the researcher to select an information-rich case and subjects to study in depth (Patton, 2002). Furthermore, this research aimed to meet the criteria for good qualitative research, such as credibility, validity, reliability and ethical issues.

\subsection{Narrative Research}

\subsubsection{Definition and characteristics of narrative research}

Narrative research is a distinct form of qualitative research (Creswell, 2005) and considered a branch of interpretive research (Gudmundsdottir, 1998). Narrative research focuses on the lives and experience of individuals in connection with social events and other people (Clandinin and Connelly, 2000; Creswell, 2005) and links individual stories together to create accounts of phenomena (Riessman, 1993). Narratives are created through relating experiences (Riessman, 1993): the natural setting does not express meaning itself but individuals construct meaning through their events and actions. The narratives allow researchers to deeply understand a natural setting by providing three main areas to explore: temporality, meaning and social contexts (Elliott, 2005).

\subsubsection{Temporality}

Narrative research is focused on experiences of individuals which they impart through storytelling (Boje, 2001; Creswell, 2005) and retrospective explanation in the form of memories (Boje, 2001). A narrative presents stories of events and actions which include a temporal dimension (Agostino, 2005). Each event that occurs is affected by past experiences, and has future implications (Riessman, 1993; Gudmundsdottir, 1995; Czarniawska, 1998). As a result, understanding past experiences can lead researchers to explore both present and future stories (Creswell, 2005).

\subsubsection{The meaning of the narrative}

Meaning is a prominent component of narrative research. A story's meaning is concerned with understanding the past actions of narrators. Storytelling is normally performed in evaluation clauses in which tellers tell a story according to how they interpret actions (Riessman, 1993). Elliott (2005) considers that the meaning of a story is established through an evaluation process which occurs with audience collaboration as they demonstrate their understanding. 


\subsubsection{Social contexts}

As mentioned previously, social reality is a complex phenomenon. Stories involve complex relationships between activities, resources and conditions. Qualitative researchers can select views to be explained (Gubrium and Holstein, 1998). Narrative researchers understand and explain stories based on contexts and structures, and expect audiences to be aware of the meaning (Riessman, 1993; Agostino, 2005).

With narrative analysis, researchers obtain rich data through story-telling by respondents and observations by researchers (Gudmundsdottir, 1995; Czarniawska, 1998; Clandinin and Connelly, 2000). The narratives present a society and its culture through individual stories (Riessman, 1993). Telling stories of past events seems to be a universal human activity (Riessman, 1993; Czarniawska, 1998). It can encourage respondents to give data, because it can make them feel proud that their stories are important (Creswell, 2005). However, people may reluctant to talk about some topics, such as political issues and painful experiences (Riessman, 1993; Elliott, 2005), or they may create stories in order to be more entertaining (Creswell, 2005). To address these issues, friendly conversation between the storyteller and the audience may reduce the distance between them and allow the narration to flow freely.

\subsubsection{Narrative research in organisation and KM research}

The narrative method has been used in many social research areas that involve people and micro level social aspects (Creswell, 2005) including organisational and information systems research. When studying organisations, the complexity of organisational culture can be revealed through the words and messages of its members. This is because narratives can express members' feelings, beliefs and sense of experience (Trice and Beyer, 1993). Experience cannot be accessed directly. Access to experience requires talking, words, interaction and interpretation (Riessman, 1993). Clandinin and Connelly (2000) mentioned that narrative is the best way to understand and represent experience, because narrative thinking is a key way to formulate experience. For instance, Fischette (2000) conducted narrative research to study learning and community organising through the development of individual empowerment and groups. Fischette used narrative inquiry to obtain the individual stories and experiences of respondents, and analysed them to find common themes. Kovan (2001) conducted narrative research to understand environmental professionals 
working in non-profit organisations by describing the individual experience of nine such workers.

As mentioned in section 3.1, information systems research focuses on understanding the context of organisations to develop suitable IS. Like IS research, IS-based KM research focuses on understanding organisations in terms of leveraging knowledge. As a result, narrative analysis can be used to investigate information systems and organisations in order to understand social interaction between people and the systems, as well as to propose better systems (Hunter, 2006). For KM research, narrative analysis can be a tool that allows researchers to gain insight into how organisational members make sense of activities, in order to study organisational knowledge (Patriotta, 2004). Aldridge (2002), for instance, investigated an organisation with narrative research to obtain subjective data about KM practice, and observed and interpreted the complex phenomena of the organisation in order to propose a multidimensional model for knowledge asset building.

\subsubsection{Narrative research for this study}

The narrative method for this research was used to study a selected organisation which is a CE: "Narrative form of organisation studies are easiest to find in case study: research cases, educational cases, and fictive cases” (Czarniawska, 1998, p.14). This research was based on Prae Pun, a community enterprise in north-east rural Thailand as a specific context to explain the phenomena of CEs. Given the objective and specific context of this research, the narrative method was suitable for this study for three main reasons as follows:

\subsubsection{To gain rich data}

Narrative research allowed the researcher to gain rich data through the specific context and respondents' stories. Individual stories present insights into a social setting (Bertaux, 1981). As mentioned previously, individual stories do not only describe past actions but contain meaning in the form of understanding the actions of storytellers. Narrative analysis is suitable for studies that focus on subjectivity and identity, exploring the characteristics of subjects (Riessman, 1993). Furthermore, narrative research provides individual stories that present the culture of studied 
organisations (Trice and Beyer, 1993), which is a major component of the theoretical framework of this study.

\subsubsection{To understand a CE in depth}

Narrative research allowed the researcher to gain deep understanding of the case study organisation through both storytelling by individual CE members and observations by the researcher herself. This is because narrative provides three main strategiestemporality, meaning and context of stories-which form a basis for the researcher's understanding. The researcher does not only gain current stories, but also background stories through the temporal nature of narrative and the intention of the actors which involve future implication.

\subsubsection{To focus on naturalistic methods}

The last issue that made narrative research suitable for this research is that it is based on naturalistic methods. Narrative research is a method of drawing out the truth behind the experience (Agostino, 2005). As mentioned above, telling stories is a common practice. Gudmundsdottir (1998) found that a story is the most natural way to elicit data and enhance the understanding of researchers. A narrative has a free flowing manner (Tan and Hunter, 2003). Telling stories is a natural activity and all respondents have stories to tell of their experiences (Creswell, 2005). Given the characteristics of Prae Pun and the culture and values of Thai society, the narrative method was appropriate. This is because most respondents in this study were comfortable expressing ideas in an informal environment within their in-group. Another crucial reason for using the narrative method in this study was because this study gathered traditional knowledge from the respondents in its original form of oral transmission (Kroma, 1995; Storey, 2005; Sukula, 2006).

Thus, narrative research allowed the researcher to obtain rich data from a selected organisation within a specific context that enhanced her in-depth understanding of its phenomena through individual stories. Compared with ethnography and grounded theory, narrative researchers collect micro-analytic pictures rather than gaining a broader view of social setting (Creswell, 2005). The researcher understands the actions of respondents with their meanings and temporality rather than contemporary phenomena, which can be obtained through the case study method (Yin, 1994). As 
qualitative research, the researcher has less control over or influences on the social setting - unlike a case study where researchers have to provide some direction when interviewing (Walsham, 1995) and action research provides an opportunity to help the organisation with problem evaluation and solution development (Bryman, 2004). Therefore, narrative research allowed the researcher to gain rich data, despite the small numbers of respondents and limited timeframe of this study.

\subsection{Research design}

Given the objectives of this study, it is divided into two parts in order to find clear data to answer the research questions. The first part aims to explore the characteristics, organisational culture and current KM practice of the community enterprise. This part focuses on ethnographic data collection methods: narrative inquiry, participant observation, and document analysis.

The second part is the phase of drawing conclusions to answer the research questions. The conclusion drawing is a process of interpretation information to ground a theory and depicting data to answer research questions (Hall and Hall, 2004). The conclusion drawing is based on propositions and theoretical frameworks which are proved by phenomena found in this research, in order to answer research questions, as suggested by Miles and Huberman (1994). Meanwhile, several researchers, such as Emory and Cooper (1991), and Zikmund (1991) suggest that propositions are statements of concepts which can be proved by phenomena. The overall research procedure is shown in figure 3.1 . 


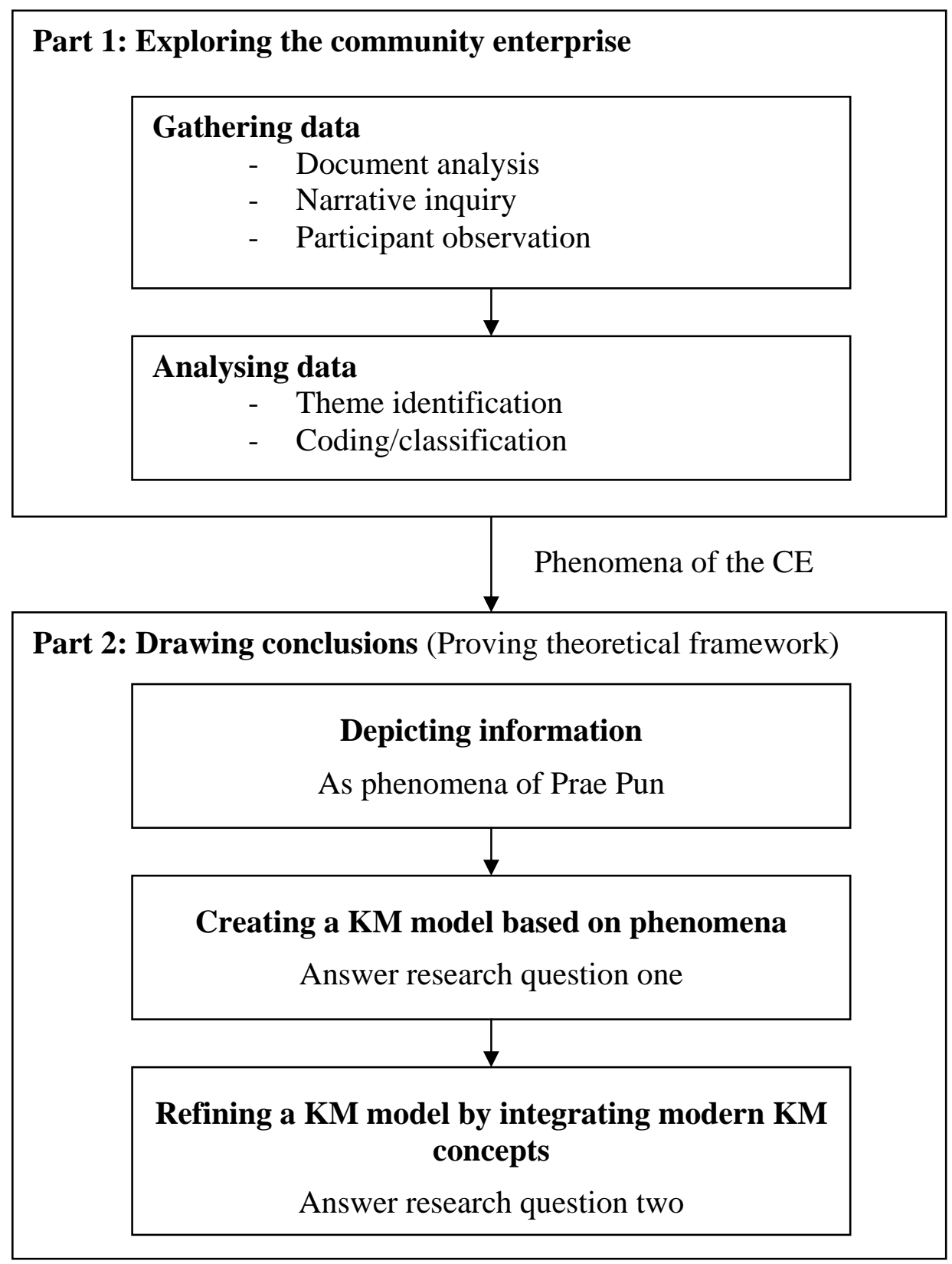

Figure 3.1 Research procedure

\subsubsection{Research site}

In order to gain in-depth understanding in a limited timeframe, this research examined a single representative case which was a specific environment where the researcher could gather contextual data. Ethnographic techniques allow researchers to understand culture and social behaviour through specific groups or a case (Punch, 2005). The research site of this study was Prae Pun-a community enterprise producing handwoven products in northeast rural Thailand. Prae Pun was selected as a research site 
based on purposive sampling. Purposive sampling involves selecting subjects who have important characteristics in which researchers are interested (Williamson, 2006). Prae Pun has the typical characteristics of CEs in developing countries, especially in Asia. Those characteristics include location, objectives, and operation systems. Prae

Pun is a community enterprise operated and managed by rural women. Prae Pun comprises around 200 women who are farmers from seven villages in four districts of Khon Kaen province. Prae Pun was established with the aims of women's and community development, income generation, natural resources conservation, and preservation of traditional hand weaving. Given such typical characteristics, Prae Pun can be used as a representative example of CEs in Asia because most of them, as mentioned in the literature review, are located in rural areas, have been established with the aim of social development, and operate with limited and local resources. Another crucial reason for choosing Prae Pun was that the researcher could quickly gain insights from respondents. Prae Pun members easily accepted the researcher as a temporary in-group member because the researcher used to work for an NGO in north-east rural Thailand, which is part of the same network as Prae Pun, and these experiences enabled her to become familiar with respondents who were rural women and NGO workers.

\subsubsection{Data collection}

According to the paradigm and method of this research, data collection is focused on narrative inquiry and ethnographic techniques for which researchers have to immerse themselves in the research site for some time. The immersing of researchers in social setting allows the researchers to get an insider perspective of events and contexts and elicit data from informant participants (Punch, 2005). This is because qualitative research requires deep understanding of complex social processes and social organisations, rather than understanding superficial patterns through large numbers of respondents (Mason, 1996). Interpretive researchers emphasise qualitative data collecting through naturalistic enquiry, which takes place in a natural setting (Silverman, 2005; Williamson, 2006). Ethnographic techniques allow researchers to obtain rich data about people (O’Connor, 2005) by observing the behaviour of respondents. There are many data collection methods classified as ethnographic techniques. Two prominent ethnographic techniques are open-ended enquiry (Williamson, 2006) and participant observation (Punch, 2005; Silverman, 2005). 
Therefore, this research used narrative inquiry, participant observation and document analysis as data collection methods to explore the phenomena of the CE. The overall process of data collection is shown in figure 3.2 and discussed in following sections.

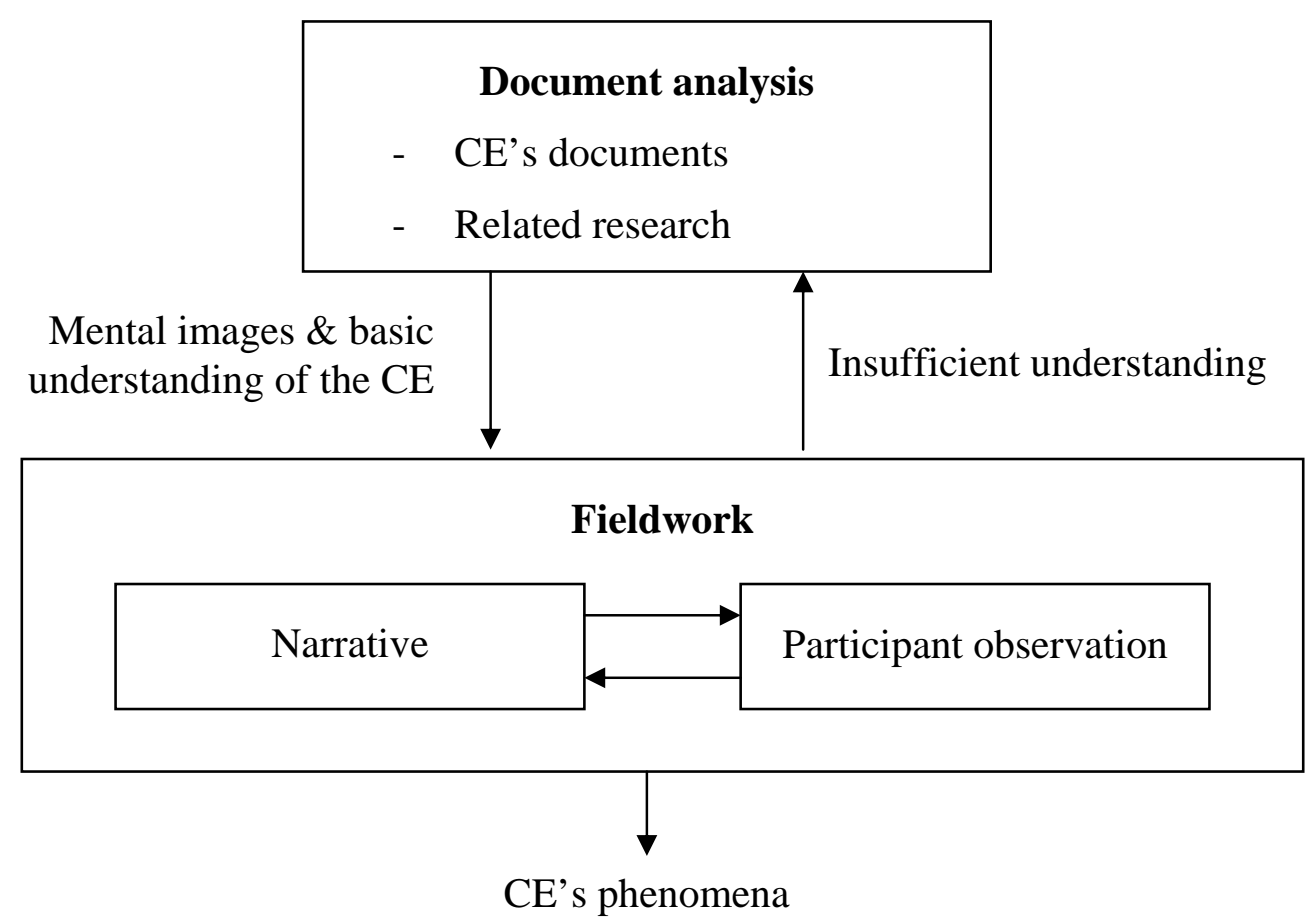

Figure 3.2 Data collection procedure

\subsubsection{Document analysis}

The data collection phase of this research began with document study before going on to the other techniques, but this also occured concurrently. This is because documents provide a mental image for researchers (Mason, 2006), and useful information and guidelines for the other data collection techniques (Frechtling and Sharp, 1997). Documents provide data or evidence that are not available through other methods and alternative dimensions of data (Mason, 2006), which can be used for triangulation (Punch, 2005). Furthermore, documents provide views that occurred before the study period (Patton, 2002). Documents are a rich source of data for social research (Punch, 2005). Documents are considered as material culture in anthropology. They provide information about particular organisations or societies (Patton, 2002), and can be either text-based or non-text-based, such as pictures, video, and graphic presentations (Punch, 2005). Texts provide aspect of sense-making activities and can make sense of social reality for researchers (Miller, 1997). The researcher started by examining 
leaflets, books written about Prae Pun, research reports and photos. Then, the researcher examined meeting reports, in-house training reports, and sales documents. Those documents provided information about many dimensions, including Prae Pun's group and business management, production processes, and knowledge acquisition and learning processes.

\subsubsection{Narrative inquiry}

Narrative inquiry is the main data collection technique. Narrative inquiry is a common and appropriate technique for organisational research (Czarniawska, 1998), and fieldwork which is concerned with culture and detail (Van Maanen, 1988). Narrative inquiry can help researchers to gain in-depth understanding and comprehensively illustrate the phenomena of social reality (Czarniawska, 1998). This is because narrative inquiry focuses on open-ended exploration (Gudmundsdottir, 1996; Conle, 2000) and is concerned with story and discourse (Chatman, 1978; Gudmundsdottir, 1995). Thus, respondents could talk about their experiences without the influence of researchers' questions. The researcher asked them to tell their stories and experiences from the beginning of their involvement with the CE until the present. The researcher did not interrupt them while they were narrating these stories, but asked them to explain emerging issues that were useful to this research in more detail, and some points that the researcher did not sufficiently understand before the end of session or in the next session. In order to get rich data from respondents, the narrative inquiry was conducted after the researcher had participated in activities with the CE's members for a few days. This allowed the researcher and the respondents to become familiar with each other, and for the researcher to find respondents who were willing to talk to and felt comfortable with the researcher. However, respondents were mainly selected for both theoretical qualifications and their willingness to participate. As with the research site selection, respondents were selected based on purposive sampling which is often used for qualitative research (Punch, 2005). This is because purposive sampling allows researchers to gain information-rich cases for study in depth (Patton, 2002). This study comprised 18 informants in order to cover all the positions in the enterprise and representatives from every village. The respondents included at least one person in each position and at least one member from each village. The enquiry took place at respondents' familiar locations, such as their homes, working places, temples and the CE's office, and in their dialect. In order to ensure that the researcher 
could obtain all relevant stories, the researcher prepared key enquiry dialogues to introduce topics in Isan and conducted a preliminary enquiry with other members in the enterprise. The preliminary enquiry helped the researcher to become familiar with Isan dialect. The enquiry was recorded by tape or electronic recording. Short notes were taken at the end of each session or the end of the day in order to pay attention to stories and avoid distracting respondents. The recordings provided detailed data which could be used to check the completeness of the data and accuracy of translation from Isan dialect to Thai language.

\subsubsection{Participant observation}

From an epistemological perspective, evidence of the social world can be obtained by observing and participating in a natural setting. Observation allows researchers to obtain descriptive data of events and behaviour (Maxwell, 1996) through social interaction in specific contexts instead of retrospective stories of people (Mason, 1996). Participant observation is a popular technique for studies of organisations or small groups, and different cultures (Yin, 1994). Participant observation refers to observation where researchers play the role of participants in a study field (Atkinson and Hmmersley, 1994). Participant observation allows observers to gain inside views and to obtain data on sensitive issues because they become temporary members of an organisation or society (Walsham, 1995). In this respect, researchers play the role of both participant and observer (Punch, 2005). Participant observation can involve some bias. There may be less accuracy when observing events because researchers become internal observers and involved in the group which leads to few opportunities for note taking (Yin, 1994). In order to reduce such bias in this study, the researcher played the role of observer-as-participant who mainly observed with little involvement in activities (Bryman, 2004; Gorman and Clayton, 2005). From this perspective, the researcher observed with partial immersion which allowed free time to review the observations (Delamont, 2004). Furthermore, some other forms of evidence such as photographs were taken to ensure accuracy of observation (Babbie, 2001).

This research used the participant observation technique in order to gain deep and complex information about the CE. The observation took place concurrently with narrative inquiry. Participant observation allowed the researcher to collect additional data by participating in the events and activities being studied. The researcher took 
brief notes during observation and wrote full field notes at the end of each day or at the end of sessions. In addition, some activities and places, such as office spaces, workplaces, work processes, daily activities and social activities, were recorded photographically. In order to avoid over generalisation which could impede enquiry, the researchers observed many representative samples without selecting specific cases, following the suggestion of Babbie (2001). During the period of data collection, the researcher participated in the enterprise's activities, such as committee meetings, members' meetings, production processes at members' houses, and marketing and selling at the enterprise's shop and handicraft fairs.

\subsubsection{Data Analysis}

There are many strategies for analysing qualitative data including analytic induction, grouped theory, narrative analysis (Bryman, 2004; Punch, 2005), abstracting and comparing, discourse analysis (Punch, 2005), and Miles and Huberman's framework (Miles and Huberman, 1994; Punch, 2005). Indeed, the basic processes of qualitative data analysis are describing and comparing phenomena by breaking down data, and classifying and connecting them in terms of concepts or theories (Dey, 1993; Punch, 2005). Describing phenomena involves interpreting the characteristics of people, objects and events studied in order to develop comprehensive descriptions of the processes and contexts of actions, and intentions of actors. Classifying data is a process of dividing data into categories based on the topics that the research is exploring. Connecting comprises the process of making accounts while referring to concepts or theory (Dey, 1993). In narrative analysis, the focus is on transferring field texts to research texts which are tidier and more usable (Clandinin and Connelly, 2000; Boje, 2001). The narratives are investigated and interpreted in order to provide logical and meaningful answers to the research questions (Riessman, 1993; Clandinin and Connelly, 2000). This can be done through coding field texts, and then sorting them into themes and conceptual or theoretical frameworks (Clandinin and Connelly, 2000; Boje, 2001). Theme analysis is a common technique used for examining qualitative data; it focuses on both deductive and inductive analysis. In other words, theme analysis focuses on categorising data based on both theories and emerging stories (Boje, 2001). Coding data into themes can help researchers in developing and extending explanations of data (Hall and Hall, 2004). In 1998, O’Callaghan reduced and analysed narrative data for her study of the social construction of pre-service 
teachers' instruction by coding into themes and categorising. In addition, Shrubsole (2003) developed major themes with which to analyse data and draw conclusions for her study of community health.

As this research used narrative inquiry and participant observation as its main data collection techniques, the field texts contained rich data and insights of respondents. This study carefully described these field texts to answer the research questions by using narrative analysis based on the three basics processes of qualitative data analysis - describing, classifying and connecting — in an iterative manner (see Figure 3.3). In this respect, Dey (1993) states that data analysis is better done in an iterative manner in which the processes of data analysis-describing, classifying and connection-do not proceed in a linear fashion. They are performed in a spiral in which researchers have to retrace their steps to previous processes in order to obtain clear understanding in accounts (see Figure 3.4).

The analysis involved coding into themes and sorting them into concepts or a theoretical framework in order to draw conclusions with which to answer the research questions. Compared with the qualitative data analysis model of Miles and Huberman (see Figure 3.5), narrative analysis presents the research texts as sorted themes in narrative form with stories rather than as quantified data. 


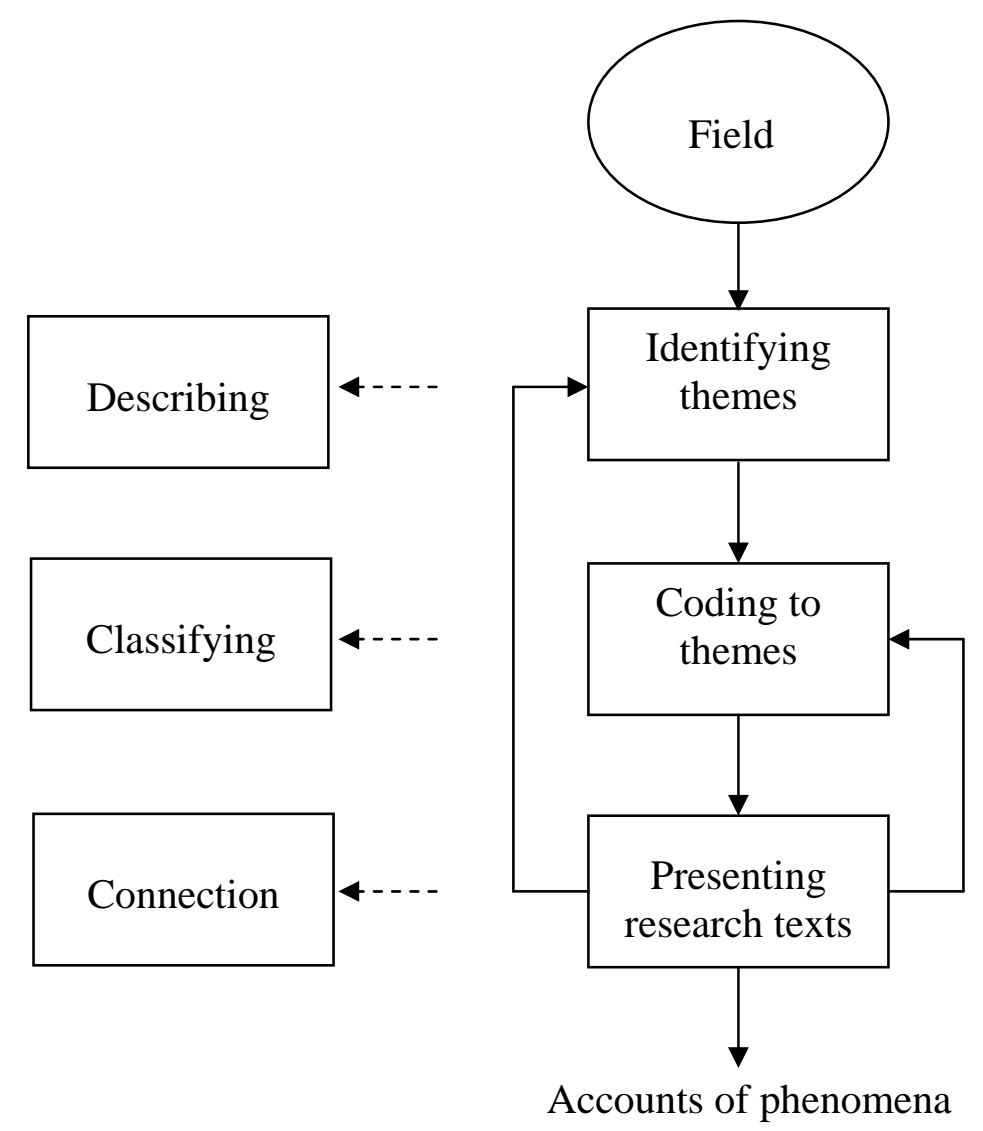

Figure 3.3 Data analysis framework of this research

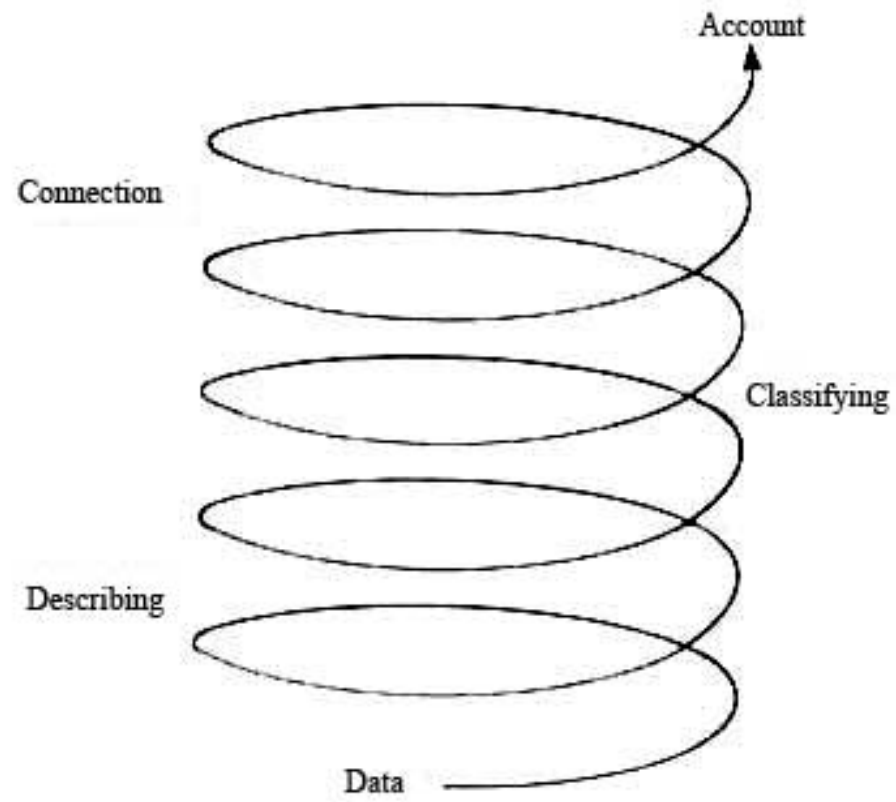

Figure 3.4 Qualitative data analysis as an iterative procedure (Dey, 1993) 


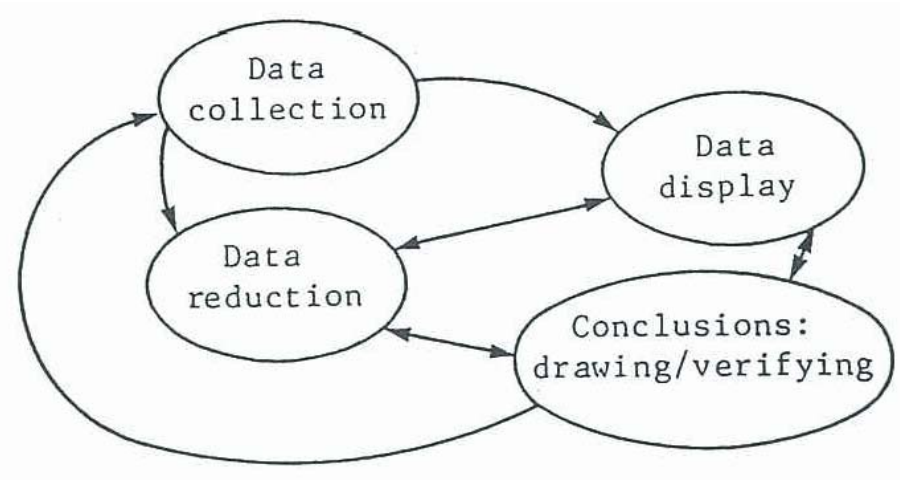

Figure 3.5 Data analysis model of Miles and Huberman (1994)

This research followed the coding process developed by Gibbs (2002). This is because Gibbs' coding system can support the data analysis framework of this research. It has the clear processes of identifying, comparing and connecting collected data with research concepts. It comprises four processes: extracting text, coding, classification, and refining codes. Coding is the process of identifying codes or names in the passages of text. Codes are designed to represent concepts. Then, codes or nodes are arranged in a hierarchy of concepts. Nodes present the relationships of the passages of text in the form of a child/parent relationship or tree structure that allows researchers to understand the perceptions of respondents and gives an overview of the conceptual framework. This is because codes or nodes are connectors to concepts or theories. Finally, code refining is the process of redesigning codes and coding (Gibbs, 2002). This refining process takes place throughout the data analysis in order to improve the accuracy of coding the passages of text, and, thus, enhance overall understanding of the material.

The final process of data analysis is conclusion drawing, which is the process of interpreting information and generating propositions or theories to achieve the objectives of the research (Miles and Huberman, 1994). In this study, the researcher connected themes together to make research texts which contained accounts of the phenomena. Research texts are the series of accounts that help readers to understand the context of the research (Creswell, 2005). Narratives are phenomena (Clandinin and Connelly, 2000) and precise information, which is subjective (Personal Narratives Group, 1989). In order to write the research texts, the researcher had to go back to major themes and classifications in order to ensure full understanding in the accounts. 
In order to answer the research questions, the texts were presented based the theoretical framework of this research. Theories or propositions are statements of concepts that can be proved by phenomena (Emory and Cooper, 1991; Zikmund, 1991).

In brief, the research texts were developed through narrative data analysis according to Dey's data analysis framework (1993). The research texts contained accounts of phenomena of the characteristics and current KM of the CE, which were employed to create a plausible KM model for the CE. The model was used to answer research questions, based on the findings in order to support the theories.

\subsection{Quality of research}

The quality of qualitative research focuses on the trustworthiness of the findings and interpretation of researchers (Glesne, 1999) in order to enhance the objectivity of research (Perakyla, 1997). There are many criteria to evaluate the quality of research including validity and credibility, the reliability of methods (Silverman, 2005) and ethical issues (Drisko, 1997).

\subsubsection{Validity}

In qualitative research, validity refers to the correctness and credibility of the descriptions, explanations and interpretations of accounts rather than the data collection methods or data. It is focused on objective reality or possible interpretations of which accounts are credible (Maxwell, 1996). Maxwell pays attention to three main areas: descriptive validity, interpretative validity and theoretical validity. On the other hand, generalisability has received less attention because generalisation of qualitative research data is based on theory which can make sense of similar situations (Maxwell, 1992).

Descriptive validity is concerned with the accuracy of accounts that researchers report and infer from what researchers see and hear during fieldwork. Meanwhile, interpretative validity is concerned with the correctness of accounts developed by the understanding of researchers through participants' meanings. Lastly, theoretical validity refers to the theoretical understanding of researchers to explain accounts with accurate concepts (Maxwell, 1992). Given this, descriptive validity and interpretative 
validity are of primary importance for interpretations and conclusions. To enhance descriptive validity, the transcriptions of the narratives of this research were translated from Isan dialect into standard Thai, and then into English, with the advice of a native speakers, as well as observation notes. In terms of interpretive validity, this research employed data triangulation which is the most common and popular technique to increase the validity of qualitative research (Bryman, 2004; Silverman, 2005). Multidata collection techniques allow researchers to dismiss unclear and misunderstood issues by checking data from any particular technique with other techniques (Bryman, 2004). This research used multiple data collection techniques, including narrative inquiry, participant observation and document analysis. The refutability principle is used to reduce anecdotal evidence which involves data from a few well-chosen examples. The refutability principle leads the researcher to explain whether phenomena are objective or subjective, using concepts or theory (Silverman, 2005). Futhermore, generalisability and transferability were considered in order to increase the validity of this research. This is because generalisability and transferability can widen the applicability of findings (Drisko, 1997). Guba and Lincoln (1994) mentioned that thick description, which contains contextual information, meanings and intentions of actors, can provide a sense of the possible transferability of findings. Thus, this research aimed for validity through generalisability and transferability through thick descriptions of accounts.

\subsubsection{Credibility}

Credibility refers to trustworthiness (Belcher, 1994) and acceptability to others (Bryman, 2004). As mentioned above, credibility is considered part of validity. It is concerned with internal validity (Bryman, 2004) which focuses on the accuracy of explanation of findings (Bryman, 2004). Credibility can be increased through thick description of raw data-respondents' own words, and context, which provides a sense of the wholeness of the event or environment-so that readers can assess the accuracy of the researcher's interpretations and conclusions (Drisko, 1997). Furthermore, credibility can be increased through confirmation by participants (Drisko, 1997; Bryman, 2004). As a result, this research ensured credibility by conducting confirmation sessions that allowed respondents to narrate particular stories on two occasions. This context can be viewed as confirmation according to Bloor (1997) because the respondents had an opportunity to re-examine the stories. 


\subsubsection{Reliability}

Reliability is concerned with consistency and stability, i.e. whether a study can be repeated again with similar results (Babbie, 2000; Bryman, 2004). In ethnographic research, reliability refers to the consistency of findings with others using the same method (Kirk and Miller, 1986). As mentioned above, this research was conducted in a social setting in which the researcher could not control the research site. Thus, this research addressed only internal reliability. Internal reliability is concerned with consistency of interpretation of the same events by different researchers or observers (Bryman, 2004). However, this research had only one observer. Therefore, this study enhanced the internal reliability of narrative inquiry by using the test-retest method, which involved obtaining the same information twice (Babbie, 2000), in order to ensure that respondents interpreted the same questions from the researcher in the same way. The key narrative inquiry dialogue was prepared in Isan dialect and checked by a native speaker in order to ensure appropriateness of wording. In addition, preliminary enquiry was undertaken with other Prae Pun members in order to test for reliability. Furthermore, data source triangulation techniques were used to ensure the reliability of information from the observation. The researcher observed the same sorts of events and work processes of the CE as many times as possible during the fieldwork period.

\subsubsection{Ethical issues}

As mentioned above, this study involved human participants. Ethical issues were addressed in order to increase the quality of this research. Drisko (1997) mentions that the maintenance of social work ethics is one of six criteria for strengthening qualitative research. Ethical issues in qualitative research are concerned with avoiding harm, consent, deception, privacy and protecting the confidentiality of data. Research subjects have a right to know the nature of research and to decide whether to participate in the research project or not. In addition, the privacy and identity of research subjects should be protected (Punch, 1994). Thus, this study conformed to the criteria of Victoria University's Human Ethics Committee. Enterprise representatives and respondents were informed about the study through participant information sheets. Each respondent had to sign the consent form or give verbal consent on a tape recording after the researcher read out participant information sheets and the consent form before engaging in the enquiry. Respondents were volunteers 
who were willing to participate in the research. As a result, the issue of trust which refers to the relationship between researcher and participants, and the responsibility of researchers not to spoil the research site for future potential research, as noted by Ryen (2004), was considered. Researchers should deal with participants by using empathy and not treating them as powerless (Ryen, 2004). The researcher gained rapport through genuine good feeling and conducted the enquiry with respondents who were comfortable and willing to share their experiences. Lastly, privacy and anonymity issues were considered from the data collection stage to the final publication of the research. Furthermore, the enterprise will receive a summary of the results of this study.

\subsection{Chapter summary}

The objectives of this research are to seek to better understand the characteristics and organisational culture of a $\mathrm{CE}$, and its current $\mathrm{KM}$ in order to present a possible $\mathrm{KM}$ model. This research employs qualitative research, in which data are collected with narrative inquiry and ethnographic techniques. The data analysis used narrative analysis to enable the researcher to understand complex phenomena in depth in order to summarise the characteristics and situations of the CE to create a KM model. This research ensured the quality of the findings by employing validity and credibility, reliability, and ethical issues. 


\section{Chapter 4 \\ Research Procedure}

This chapter focuses on the procedure of this research including the process of data gathering and data analysis. It begins with selecting a community enterprise, access to respondents, roles of the researcher, data gathering, data analyses and data presentation.

\subsection{Research site selection and access}

The research site was selected with a purposeful sampling strategy in order to gain access to an organisation having typical characteristics of general community enterprises (CEs). Prae Pun was selected because it has characteristics typical of other CEs. Prae Pun is owned and operated by local community members with the major objective of community development. Its businesses rely on local resources, including human resources, financial resources and knowledge. Another reason is that Prae Pun is located in a rural area like most Asian CEs, as discussed in the literature review. The final reason is because Prae Pun is located in northeast rural Thailand, an area in which the researcher has local experience. Given the time limits of a study, choosing a research site relevant to the researcher's background can help the researcher become familiar more quickly with respondents.

The researcher gained access to Prae Pun by presenting her research intention and motivation for doing this research to an advisory committee member of the CE. Then, she forwarded her intentions to a Prae Pun manager and the board committee of Prae Pun. The manager and the committee accepted the researcher to conduct the research at their organisation. Access to the research site through the advisory committee member was an advantage, which meant the researcher could save time in gaining both acceptance, and familiarity with the research site and respondents. The personal connection enabled the organisation's members to trust the researcher and treat her in a friendly manner, as the researcher is a friend of the advisory committee members and used to work with an NGO worker in this region. The researcher's background was an advantage that resulted in the respondents being willing to tell their stories to the researcher, to be comfortable with and to trust her. 


\subsection{Access to respondents}

After the manager and the board committee accepted the researcher to conduct the research at their organisation, the researcher was introduced to office staff members and the seven village groups by the manager and the advisory committee member. Before going to each village group, the researcher had a chance to socialise with the manager and office staff members. That was a good opportunity for the researcher to give more information about the research and herself to the manager, who would help to convince members to participate in this research. However, the respondents participated willingly in this research as volunteers and with self-consent.

The introductions took place on the ground floor of the village group committee members' houses in each village. The manager organised some committee members and key members of each village group for her introductory meetings. The advisory committee member gave brief information about the researcher and the purpose of her visit. Then, the researcher introduced herself and her research, and answered questions from the participants of the meetings. After that, the participants chose two or three members from each village group as respondents. These were either members who could be classified as committee members or general members. The respondent selection of this research is based on purposeful sampling, thus, the researcher deliberately included representatives of all positions in Prae Pun from every village group who were active members, in order to gain information-rich responses.

After the introductions, the researcher contacted the chosen members by telephone and visiting their homes. Of the 18 chosen, three were not available as they were busy or unwilling to participate. Significantly, the host family and respondents who were earlier interviewed were helpful in encouraging the rest of the respondents to participate in this research. To cover all the positions of Prae Pun, office staff members and an advisory member were also invited to be respondents. This is because the members of Prae Pun are classified as general members, committee members, advisory committee members, and office staff members. Altogether, there were 18 respondents, comprising eight members from the seven village groups, seven committee members from six village groups, two office staff members and one advisory committee member. 
To address ethical issues, all respondents had a chance to read the participant information sheet and ask questions before beginning, except one respondent who had difficulty in reading. The researcher read out the information sheet and consent form to her, and gave her time to ask questions and think before giving verbal consent on a tape recording. The ethical issue documents can be seen in appendix A. To respect confidentiality, although Prae Pun has a few male members, all respondents were given female Thai pseudonyms. The list of respondents' pseudonyms with their positions and village groups is given in appendix B. The researcher will also not present the hand weaving and natural dyeing techniques described by particular respondents in the research texts, because many respondents mentioned the competitiveness of the hand-woven cloth businesses. Furthermore, this research paid attention to the issue of trust-responsibility of the researcher to establish rapport with respondents in order to maintain a good impression and respectful manner. According to Thai culture, respondents who provide information should be respected because they are expert and more senior, and are considered as having higher power than the researcher.

\subsection{Roles of the researcher}

Since this narrative research used ethnographic techniques for data collection, the researcher immersed herself in the research site for a while before gathering data. The researcher acted and was treated as a temporary member of Prae Pun during fieldwork. By doing so, the researcher acted as a friend and relative of respondents. This is because Prae Pun members have a sense of belonging to the same community and members have the feeling of being relatives of each other (see chapter 6). As suggested by Fetterman (1991), the researcher stayed in the Prae Pun community and was treated with respect, friendliness and familiarity. Furthermore, as a temporary member, the researcher paid homage, helped people and avoided being arrogant, as suggested by Taylor and Bogdan (1984). The researcher stayed with one member family in a village located the middle of the village groups. She visited the other village groups several times before starting the narrative inquiry in order to be familiar with respondents. She did routine work such as cleaning and cooking with the host family, and did simple hand weaving tasks when she visited respondents' and members' houses. At the office and showroom, she stayed with a sales staff member, and helped to sell cloth during office hours and to clean the office after office hours. 
The researcher played the role of audience, collaborator and facilitator while conducting narrative inquiry but did not interrupt or show bias towards her own interests while the respondents were telling stories. For encouragement, the researcher asked questions when respondents could not think of a topic to talk about, as suggested by some experts, such as Mishler (1986) and Riessman (1993). Furthermore, the researcher had to play the role of a participant in the research site in order to observe the activities and culture of Prae Pun and the respondents. However, the researcher was an observer-as-participant who mainly observed activities with little involvement and participation, as suggested by Gorman and Clayton (2005). Thus, the researcher had more opportunities to get stories about the natural environment from the respondents and other members when the researcher stayed at the villages and CE office.

\subsection{Data gathering}

The data for this research were gathered through the narrative inquiry technique, participant observation and document study.

\subsubsection{Document study}

The data gathering began with document study before going on to the other techniques, but this technique also occurred concurrently. As discussed in chapter 3, documents provide useful information and guidelines for other data collection techniques and corroborative information to verify the data from other sources. The beginning of the data collection focused on documents that provided an overview of Prae Pun, including management systems, activities, key staff members and members in order to draw a mental image of the CE and prepare for the inquiry and observation. The researcher examined the Prae Pun leaflet, books written about Prae Pun and research reports at the initial phase of data gathering. Second, during the narrative inquiry and observations, the researcher studied more documents to gain data to enhance sufficient understanding from the narratives and observation and to provide evidence for triangulation in order to draw conclusions to answer the research questions. Committee meeting reports, in-house training reports, sales documents and research reports were studied. Each document provided information as follows: 


\section{Leaflet}

The Prae Pun leaflet provides overview information of Prae Pun including the CE's mission, objectives, main products, management systems and the location of each village group.

\section{Books}

There are two books written about Prae Pun. The first book-10 Years of Prae Pun: how to establish the hand woven cloth community enterprise-provides an overview of Prae Pun in terms of management, products and production, and lessons over the ten years of operation. Another book-Knowledge of hand woven cloth with natural dyes - Life and soul of female handicraft makers: A case study of Prae Pun-provides information of hand woven and natural dyes. The first book was useful for checking data from narratives. Meanwhile, the second book helped the researcher gain a sufficient understanding of hand woven and natural dyes and showed the expertise or knowledge possessed by each village group.

\section{Research reports}

Prae Pun has been the research site of several master theses in the fields of social development and business management. There are many thesis reports at Prae Pun office. At the initial stage of data gathering, the researcher mainly studied the chapters introducing Prae Pun in the thesis reports in order to get overview. After that, other parts of the thesis reports were studied again in order to confirm the stories from narratives. This is because most of the research provided details of Prae Pun activities through in-depth interviews with members from seven village groups of Prae Pun.

\section{Photos}

Photos provide an overview of the activities of Prae Pun conducted before this research study. Those photos helped the researcher to create a mental image of the research site visited and the styles of Prae Pun activities. Photos are also evidence to confirm particular stories from respondents.

\section{Committee meeting reports}

Committee meeting reports provided some information about the group management and policies of Prae Pun, such as production policies and member development 
programmes. Some hand weaving techniques also can be found in the committee meeting reports, because committee members also discussed hand weaving and natural dye techniques at meetings. That information provides some stories which took place before the data collection period and also confirms the stories from narrators.

\section{Training and group experimentation reports}

The group experiment reports provide mainly technical knowledge of the natural dyes which result from the experiment. That information provides understanding of natural dyes that helped the researcher in sufficiently understanding the narratives from respondents.

\section{Sales documents}

Sales documents include leaflets, catalogues and order forms which provided information of products and the marketing system. That information confirmed stories from narrators and helped the researcher gain a sufficient understanding of the stories.

Given this, documents helped the researcher in getting started to gain an overview of Prae Pun and members. That information guided the researcher in the design of the main topics for narrative inquiry and the issues to be observed. In terms of location of village groups, that information guided the researcher to set up a plan to visit respondents in each village group located in the same sub-district and district at the same time. This is because those villages are close to each other and the respondents in those groups are familiar with each other. Thus, that helped the researcher become familiar more quickly with the respondents in each area. Those documents helped the researcher to a sufficient understanding of the stories and to extend the inquiry of respondents. The documents also confirmed the stories narrated by respondents. Furthermore, those documents provided a sense of a community enterprise which focuses on traditional knowledge operated by local people. That helped the researcher to do field work with empathy.

\subsubsection{Narrative inquiry}

The narrative inquiry took place after the researcher was immersed in villages and the office, and became familiar with respondents. The inquiry occurred based on open- 
ended exploration in a comfortable environment, such as respondents' homes and weaving places, using Isan dialect.

As mentioned in section 4.3, in order to gain familiar with respondents, the researcher stayed in each village and at the office before and during data gathering, and participated in some family and community activities including housework, hand weaving and local festivals. At the office, the researcher stayed with a salesperson and participated in sales activities, and had lunch and dinner with the office staff members. This enabled the researcher and the respondents to get used to each other, and allowed the researcher to get rich data through natural conversation and observations.

The researcher asked respondents to tell their stories and experiences from the beginning of their involvement with the CE until the present. The researcher had to introduce the topics of narration by asking some questions, in order to introduce the topics when respondents did not know what stories to tell. Most respondents had difficulty in starting their storytelling, because they had a lot of stories and experiences and did not know which ones they should tell the researcher. Another reason to ask questions was that they were not confident that their stories and experiences would be useful for the research. Asking questions can help respondents to extend their stories (Mishler, 1986; Riessman, 1993). Another strategy was to ask them to show their cloth, as then they could recall many stories and narrate naturally. This is because cloth is a prominent theme of their stories and experience.

In the case of office staff members and the advisory committee member, the narratives took place at the office during and after office hours. The inquiry was also conducted in Isan dialect, except with the advisory committee member, as she is not an Isan person. Even though the researcher had experience in the Isan region for some years, in order to ensure that the researcher used the right sentences for opening questions, the researcher prepared key inquiry dialogues in Isan and conducted a preliminary inquiry with office staff members who are also members of village groups. The preliminary inquiry helped the researcher to become familiar with the Isan dialect. The inquiry was recorded on tape in order to provide detailed data which was used to check the completeness of the data and accuracy of translation from Isan 
dialect into Thai language. The records of the first round of inquiry were transcribed while the researcher was in the field. This allowed the researcher to go through the transcripts in order to capture topics for the second round of visiting. As mentioned in section 3.5.2, in order to ensure the credibility of this research, the narrative inquiries were conducted twice as confirmation sessions. Furthermore, the second round of narrative inquiry allowed the researcher to ask particular respondents to clarify insufficient understanding of topics. However, the researcher conducted second round inquiry with only some respondents due to limited time.

\subsubsection{Participant observation}

This research used the participant observation technique in order to gain more information about the CE. The researcher partially immersed herself in the Prae Pun community during the data collection period in order to review the observations and revisit the research site several times. The researcher also participated in the enterprise's activities including a committee meeting, production processes at members' houses, and marketing and selling at the showroom and handicraft fairs. In order to reduce bias, the researcher played the role of observer-as-participant (see section 4.3). The researcher took brief notes during observations and wrote full field notes at the end of each day or at the end of sessions. In addition, some activities and events were recorded photographically, such as office spaces, workplaces, and work processes.

\subsection{Data analysis}

This research reduced complex field texts to address the research concept by narrative analysis of respondents' stories through coding into major themes. Then, the results presenting the phenomena and characteristics of Prae Pun were utilised to develop the KM model and support the theoretical framework in order to answer the research questions. The overall procedure to analyse the narrative data of this study included four steps: transcribing, identifying themes, coding for themes, and presenting stories as research texts (see Figure 4.1). 


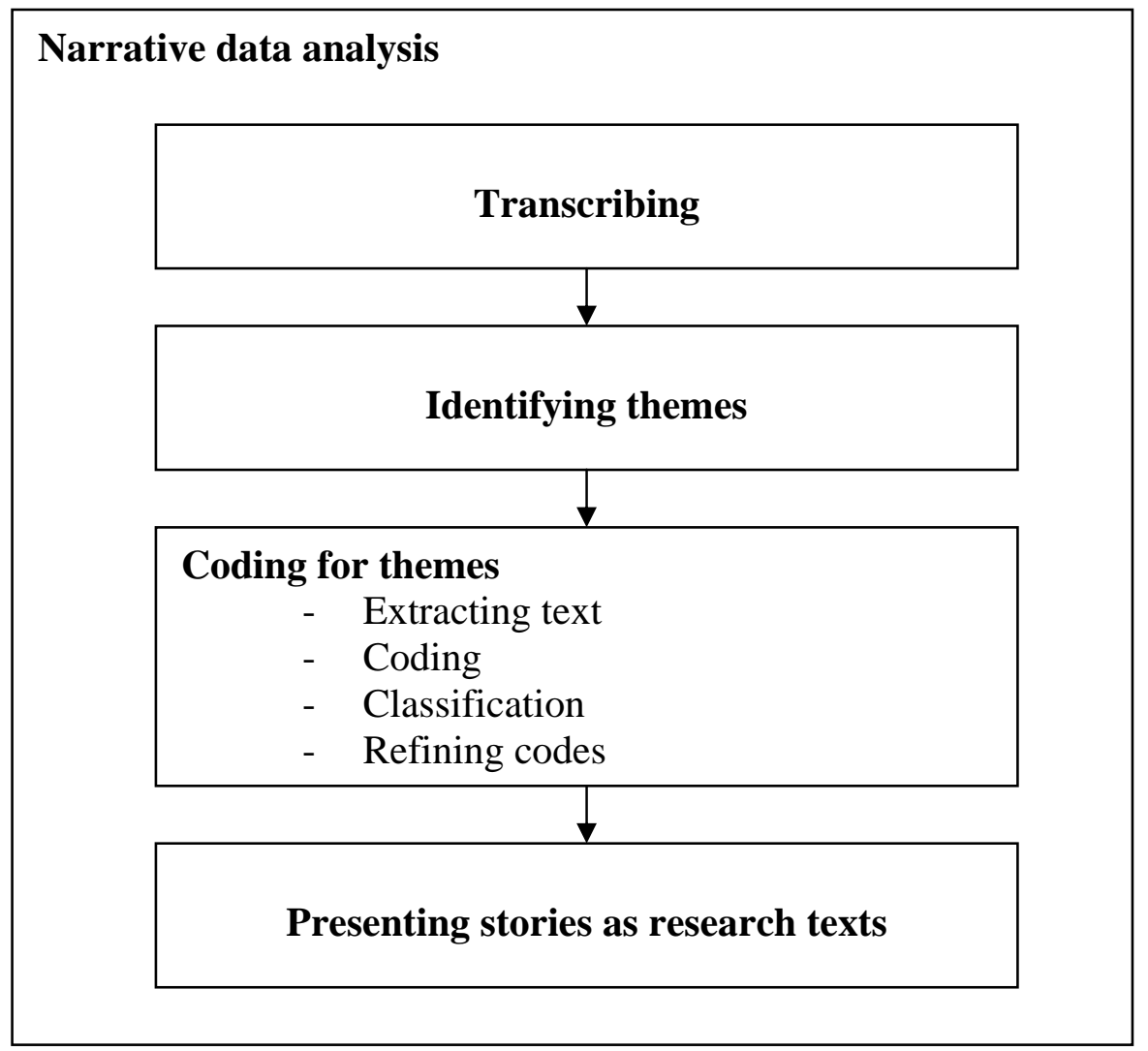

Figure 4.1 Procedures of narrative data analysis

\subsubsection{Transcribing}

Because most data were collected in Isan dialect, the data were transcribed into Thai. During transcription, the researcher obtained advice from native speakers on some Isan words and expressions that the researcher did not fully understand.

\subsubsection{Identifying themes}

Identifying themes is the process of carefully considering stories in order to understand insights and find themes or categories of data (Creswell, 2005). At this stage, the researcher read over the field texts several times in order to understand the stories and emerging themes. Two broad themes emerged from both the stories and theoretical framework, in two categories. The first category related to the enterprise as a whole, and the second to the characteristics of its KM. 


\subsubsection{Coding}

This research followed the coding system of Gibbs (2002), which comprises four processes: extracting text, coding, classification, and refining codes. In accordance with Gibbs's framework, the field texts were extracted into small passages based on the broad themes. Each broad theme was declared as a class of code, which had related nodes as subclasses to link small passages together again, in order to render stories in research text format. Small passages were assigned with codes designed to represent concepts and connect field texts. Code refining took place throughout the data reduction process in order to improve the accuracy of coding the passages of text, and, thus, enhance overall understanding of the material. Sub classes of codes of each broad theme can be seen in appendix D.

In order to facilitate grouping and classification of themes, passages or narrative fragments were converted from a word processing format into the structured format of a spreadsheet.

\subsubsection{Data presentation}

The analysed and coded data were combined in order to give the overall phenomena of Prae Pun as research texts. Narrative fragments which illustrated emergent themes were selected for presentation and translated into English. The phenomena found in this research were then used to create a model of Prae Pun's KM practice.

\subsection{Chapter summary}

Prae Pun—a community enterprise in rural Thailand—was selected to be the research site. The 18 respondents were representative of the CE's various membership categories: general members, committee members, advisory committee members and office staff members. The data were gathered by narrative inquiry, participant observation and document analysis, and analysed by coding into major themes. The analysed data were presented as research texts to support the theoretical framework in order to answer the research questions. 


\section{Chapter 5 \\ Prae Pun's stories}

This chapter will describe the characteristics that represent Prae Pun as a knowledge intensive enterprise, through stories from 18 respondents, conversations with the CE's members and my own observations. The description of the characteristics is focused on the knowledge possession of Prae Pun, its activities as knowledge processes, and the characteristics that support its KM. Those characteristics will be described through three scenes - village groups, office work, and advisory committee members’ views.

\subsection{Village Groups}

The researcher was introduced to a Prae Pun manager at the CE's office in Khon Kaen city by a friend who is an advisory committee member. The manager gave the reseaarcher a Prae Pun leaflet and photo albums to look at in order to become familiar with Prae Pun before going to the villages. The researcher had to roughly explore the common information, such as major products, location and chairperson of each village group and saw their photo before going to each village. After they had lunch together, the manager and advisory committee member took the researcher to visit the seven villages in order to introduce her to every village group. After that the researcher visited each village by herself and stayed with some members' families in order to save time travelling from the city.

Driving by car from Khon Kaen city to the west for about 20 minutes leads to the first village group at Laokwienhuk village, Muang district. Figure 5.1 shows the location and routes for reaching the seven village groups. The Laokwienhuk village group mainly produces traditional banners with ' $k i t$ ' patterns. If one drives 30 minutes further on the Khon Kaen-Chum Phae route, one will reach two village groups in Nong Ruea district — Fangnoi and Nonthong villages. Fangnoi members mainly weave kit scarves, shawls and bags. Meanwhile, the Nonthong village group is the only group weaving silk cloth. Then, a bit further north of Nong Ruea, is the Phu Wiang district. In this district are located three village groups-Huafai, Nongthum and Nongyanlan. The Huafai village group has expertise in weaving large blankets, towels and rugs. In other words, members in this village group are skilful at float 
pattern weaving, which is the pattern for traditional blankets (see appendix E). The members at Nongthum village group mainly weave loincloths, plaid and strip cloth. Meanwhile, members at Nongyanlan mainly weave place mats and tablecloths with kit patterns. The last village group of Prae Pun is the Suksomboon village group, located in Chum Phae district in the far west of Khon Kaen province. The members of the Suksomboon village group mainly weave mudmee cloth and are known to be good at dyeing, because their village has rusty water from a deep well.

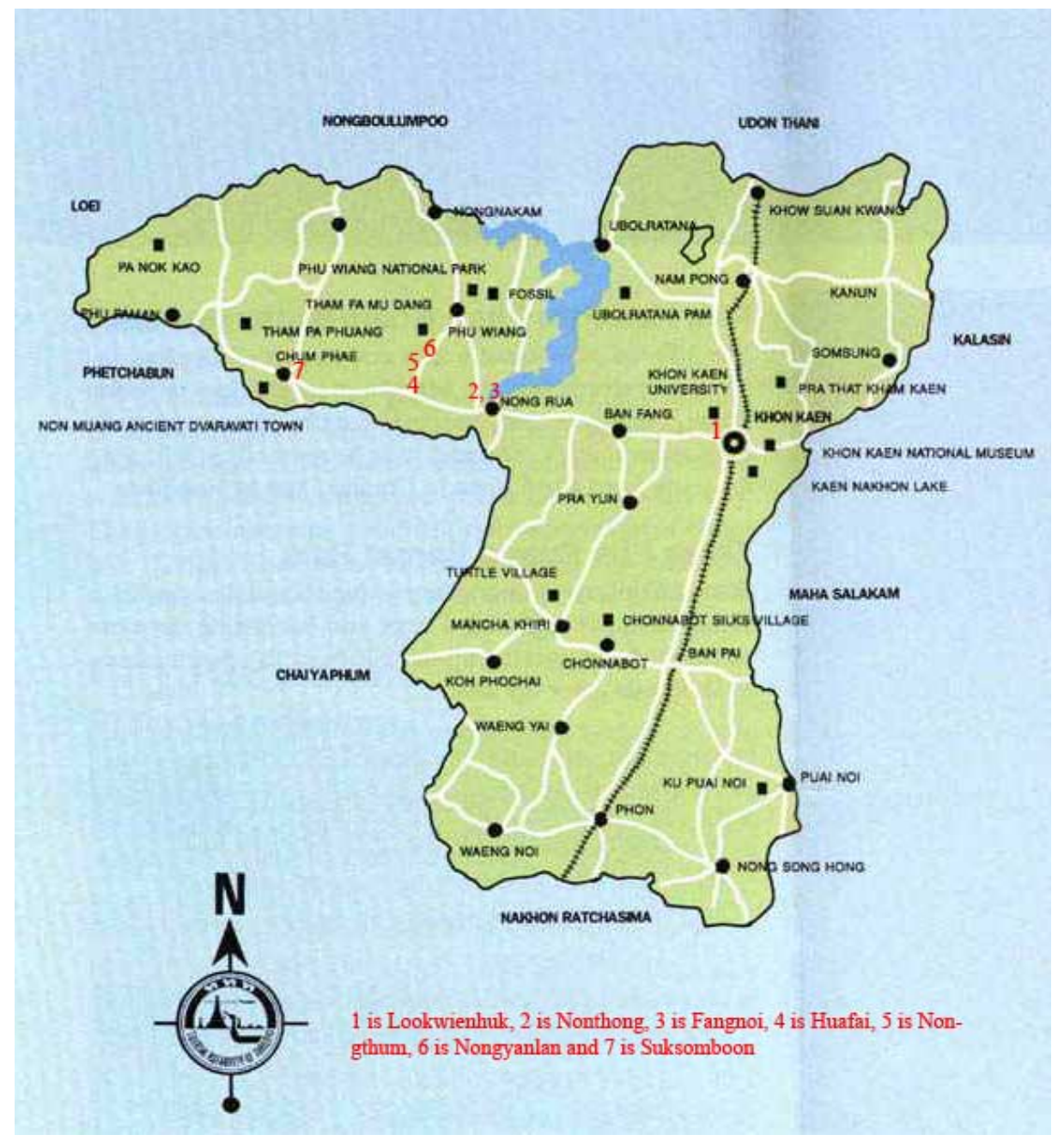

Figure 5.1 Khon Kaen map with indicators for Prae Pun village groups (Picture from http://www.khonkaencricket.com/images/kkarea1b\%20shrunk.jpg)

\subsubsection{Being Members}

In summer, along the route from Khon Kaen to all the village groups, can be seen dry paddy fields and villagers cutting grass to feed their cattle at home. This is because 
most of the land in this area can be cultivated only once a year. At the villages, on days of the year that are free from cultivation, villagers relax in the open areas of their houses, chatting, taking care of children, doing handicrafts and weaving, and having lunch together with their neighbours. The ground floor of Isan households has some open space for a hand weaving loom and a traditional bench for dining, relaxing and welcoming guests. Other households have a small hut beside the house for weaving and relaxing (see figure 5.2).

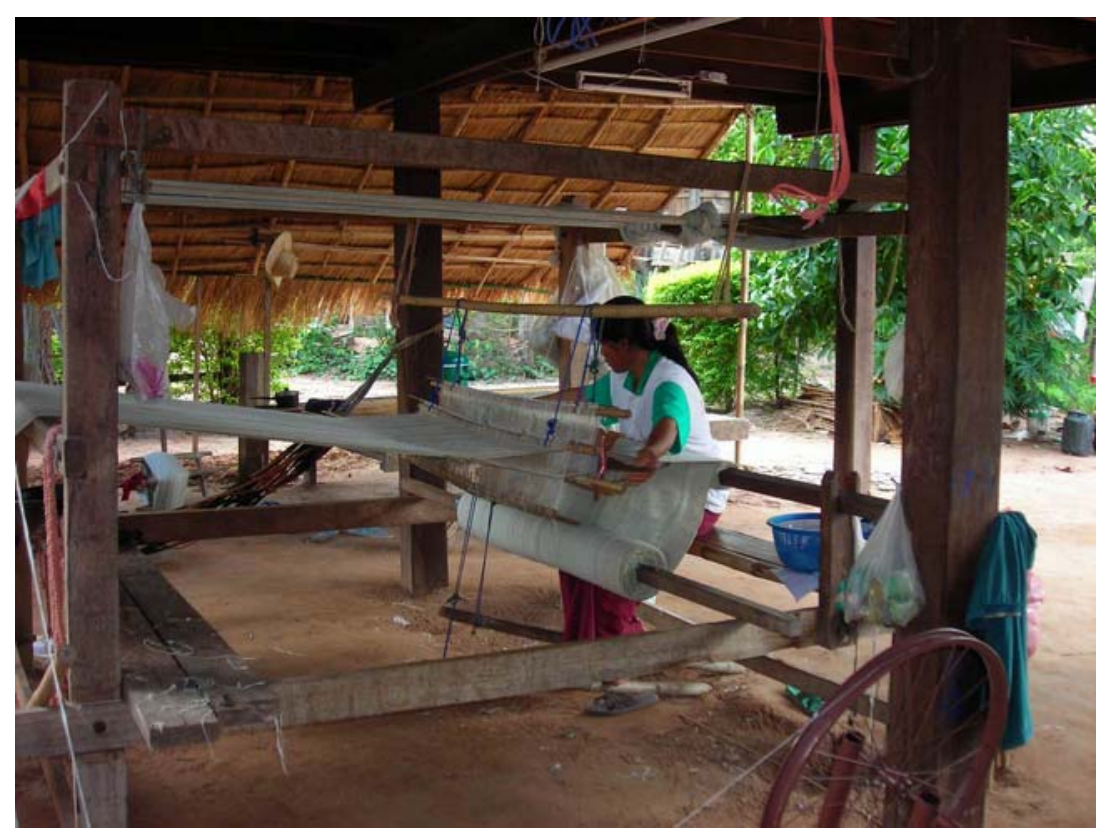

Figure 5.2 Khon Kaen village life style in summer: woman hand weaving on ground floor beside relaxing area

The researcher visited Prae Pun members' villages a little before the beginning of a cultivation season, so saw many women busy with weaving at their homes. At the host family that the researcher stayed with while doing the field work, the host mother got up early to prepare food for families, dress her granddaughter for school and tidy the home. Then, she spent most of her time weaving until time for cooking dinner. However, during the day, she had breaks to chat with neighbours and have a quick lunch with her husband and neighbours. Other members behaved similarly. Some members had a longer break during the day to water cattle in a paddy field or to visit neighbours. The host mother told the researcher that she can earn enough income from weaving to support the family and hires someone to plant rice in order to finish 
it as soon as possible. Then, she spent most of her time weaving. She told the researcher that she has to weave as much as possible in order to earn money to support her son who is studying at a university in Bangkok.

Farm work is the priority for rural people, because it is their main income and provides food for their families. At Nonthong village, Malee had a quick talk with the researcher in order to make an appointment to tell her stories, because she was in a hurry to go to her paddy field with her husband. This is because there was water on her land, so she had to prepare seedling beds and do sowing. Onanong said that Prae Pun wants members who love weaving and can sit for a long time weaving. In fact, it is hard to find those kinds of people because rural women are busy with families and farm work. Furthermore, their cultivation relies on nature, thus, they have to put aside Prae Pun activities in the cultivation season. However, beside farm work and taking care of children, rural women have to earn extra income to support families when they are free from farm work. Although one member at Loakiewnhuk village stopped weaving to do construction work with her husband, many women choose handweaving as a supplementary job instead of employment outside villages, Many members said that, currently, there are many hand weaving groups in Isan villages. There are a lot of cheap hand weaving products in markets, especially Phu Weing market (local market), but being Prae Pun members, they can get good prices and regular income for their products. Walai said that

I can earn enough money to take care of my children. I would have had difficulty in buying food, if I hadn't woven. Mae Suwit uses weaving income to support her children education to get degrees... some of us who have stopped working as employees weave at home. We can weave after farm work in the evening.

Hand weaving allows Isan women to earn income at the same time as staying at home to take care of their families and farm. Being members of the Prae Pun CE, they can get better income and quality of life. Furthermore, they can fulfil their self-esteem and self-actualisation needs. Doing hand weaving is more than earning extra income. Many members stated that they are happy when they weave. Walai mentioned that she enjoys weaving kit (supplementary weft weaving cloth) because its designs are 
pictures. Similar to Walai, Onanong said that she is happy when she does every thing related to silk preparation. Meanwhile, Kunya stated that Prae Pun gives her more than just income to support her family. She can expand her world view when she has the chance to attend a study tour.

Prae Pun not only provides regular income for members but also gives them opportunities to be business owners. Prae Pun has been registered as a co-operative, and its members are the owners of the enterprise. Members are entitled to dividends every year, but do not regularly get financial dividends. Instead, Prae Pun uses the dividends for business development and membership benefit programmes. Prae Pun has tried to develop the quality of life of rural women through using a self-reliance concept. Prae Pun uses its profit to support this with many programmes, including health insurance, senior funding, student funding and membership loans:

We decided to withdraw money for membership loans with low interest rates. They borrow money to buy land, build house and buy cattle. So, 500,000 baht could help the villagers a lot. They have their own land and are happy with this project (Kannika).

Over the 10 years Prae Pun has been operating, many in the seven villages have improved their quality of life. From my experience, 10 years ago, most poor Isan villages had simple houses where the floor and walls were made of bamboo or simple wood, and the roof was made of 'yaka' (grass leaves). Currently, most households in the seven villages of Prae Pun are well-constructed of wood and cement. Kannika said that “at the beginning, they were very poor, even their house didn't have walls. After 10 years, they have become better. Their children have higher education because of income from Prae Pun”.

Many members told Prae Pun's stories with pride. They not only obtain a better quality of life with the income from cloth and Prae Pun's benefits services, but they are confident and have an attitude of 'can-do' and self-reliance. Kannika explained that they are proud because they do not rely on anyone. They have a sense of ownership and hard work. They operate the business with self-reliance and unity. Walai told the researcher that "the committee members teach us self-learning in order 
to be able to weave”. Meanwhile, Parichat said with pride that Prae Pun was able to maintain its business during the economic crisis period whereas many other community enterprises collapsed. Nowadays, most Prae Pun members are confident. Most members talked with the researcher without the traditional Thai attitudes shyness and 'kreng jai' (consideration). Generally, female villagers are shy to talk with guests who have a higher level of education, such as government officers, NGO workers and scholars. Nevertheless, Prae Pun's members have opportunities to explore external villages through the enterprise's activities, such as study tours and handicraft fairs. Nowadays, they realise that they can operate the businesses by themselves. Previously, they thought that they would not be able to do business:

I see that they can do business because they work together as a group with regulations and discipline... They can do because they have opportunities to do. We try to create the sense of ownership for them. They have practised, because they are owners and do not want anyone to take over... They can do because they have self-confidence and ownership (Kannika).

It can be seen that Prae Pun provides members with many benefits which can be considered as incentives. Most members worked hard and searched for new techniques to improve the quality of cloth. Even though weaving at home is flexible and informal work, they have to work hard to meet Prae Pun requirements and cloth quality standards. At Nongthum village, the researcher visited one new member while she was busy with weaving. She talked with the researcher while weaving because it was close to cloth delivery day. She said there were a lot of women in this village who applied to be Prae Pun members. So, the committee members had to select by lot. She prayed in order to be drawn and she was lucky. She really wanted to be a Prae Pun member in order to earn income to support her family. Since becoming a member, she works hard in order to produce good quality cloth. Members can receive cash on cloth delivery days if their cloth met Prae Pun's cloth standards. If their cloth is of lower standard, they have to sell it on consignment. However, new members receive supervision from skilful members about meeting the standards. This topic will be expanded on further later in this chapter. 
Members have to deliver cloth regularly to the office with a certain quota for each member. At Huafai village, Raweewan told the researcher her stories while she was weaving on the ground floor. She was in a hurry to finish weaving, because the cloth was to be delivered in the next few days. She explained that the cloth is delivered to the office at the beginning of each month. The office set a monthly quota for weaving but she cannot meet the quota, because she is busy with farm work. While listening to Raweewan, her neighbour, who is also a Prae Pun member, came to share her stories and invited the researcher to visit her place. The researcher visited her and also walked around the village to to observe, become familiar with and make appointments with other members. The researcher did the same in other Prae Pun members' villages. In Isan villages, visiting neighbours for socialisation is quite common. Their houses are located in groups without fences or with open-gated fences that encourage interaction and socialisation with each other. The researcher could walk through from one house to another house without having to go out through a gate. Most villagers said greetings. Many Prae Pun members stayed at home when they knew that the researcher was visiting their village in order to learn about their hand weaving, invited her for lunch, and showed her their weaving. Villagers were very friendly and eager to share information.

During my visit, the researcher also saw members ask their daughters to help them while they were busy with preparing looms or thread, and doing farm work. On the ground floor of Urai's house, Urai rested on a traditional bench while her daughter was weaving. She gave advice when her daughter had problems. On another day, she asked her daughter to continue preparing thread which she had already started. . In this way, the daughter could follow her pattern. She said that "my daughter has woven for three to four years but she cannot khondai (warp thread preparing). I just teach her in order to let her know how to fix problems when warp threads are cut” (Urai).

It can be seen from Urai's story that the home-based system is flexible. Members can earn income with the help from family, and the home-based system also allows knowledge transfer from senior members to the younger generation. This kind of method is referred to as the traditional knowledge transfer of Isan. Most members learned basic hand weaving from their mothers when they were young. 
Nevertheless, many members commented that, nowadays, young people go to school and then work in Bangkok. Generally, however, they will come back to their home villages when they are married in order to settle down, do farm work and take care of children:

When I was a teenager, I didn't want to be a member of Prae Pun. I wanted to work in Bangkok. I came back home when I got married. I came back to settle down, do farming and take care of my kids. After my kids grew up, I joined the group because I didn't want to be a worker transplanting and weeding in the sugarcane or tapioca fields (Raweewan).

From the stories, a CE like Prae Pun offers a solution for rural women who want to earn money to support families, stay at home taking care of families, and fulfil their self-esteem and self-actualisation needs. The benefits provided by Prae Pun can be referred to as incentives for them to work hard and continue learning. As part of the village life style, members possess at least basic hand weaving knowledge and and develop specific skills at Prae Pun. They also transfer such knowledge to the new members through working. Prae Pun also has other activities that contribute to knowledge processes which will be explored later in this chapter.

\subsubsection{Cloth and Weaving}

At each village, the researcher saw members weaving different types of cloth and different sizes of reed hanging at their weaving places. At Huafai village, some members weave plain cloth and some weave rugs, but most of them have the wide reeds for big blanket weaving hanging at their weaving places. Raweewan said that

I used to weave big blankets with a grandma but now she is busy with her grandson. Now, I’ve to weave them with another grandma. She used to weave it with her daughter, but now her daughter has gone to work. We can work well together, whereas some members have to practise for a long time in order to weave together, because they couldn't pull a reed together... these village members mainly weave big blankets because other villages don't want to do... We have skills 
in blanket weaving because we used to weave blankets for our own consumption but they are simple pattern weaving with '2-kao' ...Each blanket weaver has her/his own loom(s) which have specific designs.

Raweewan took the researcher to visit her neighbour in order to show the researcher a rug loom. She explained that it is made using the same technique as for blanket weaving but is smaller. Rug weaving requires a specific thread preparing technique and a particular type of reed. They discovered the techniques by working together:

We started weaving rugs because the office ordered us to weave. We made them with trial and error. At the beginning, the rugs did not have patterns. Only wooden reeds can make patterns for rugs. We found a technique to make rugs, because grandma's rugs had a pattern. She was the only member using wooden reeds (Raweewan).

It can be seen that the members at Huafai village are good at float pattern weaving with the '4-kao' technique. 4-kao is an advanced weaving technique that requires skill at controlling four foot pedals in order to make complicated float patterns. Weaving a big blanket requires higher skills, because two weavers have to control four foot pedals each and pull wide reed together at the same time. Big blankets and rugs have the same pattern but differ in size and have some specific techniques. Figure 5.3 shows the four foot pedals for float pattern rug weaving.

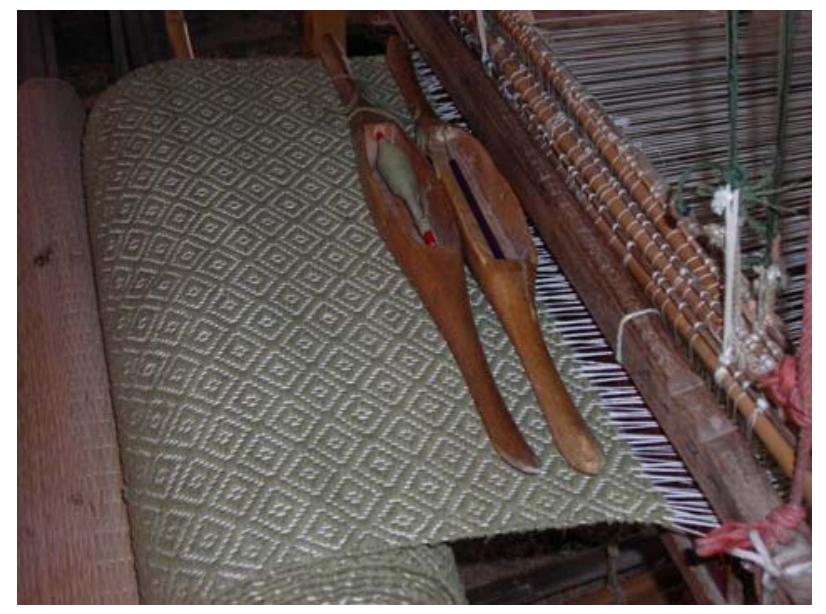

Float pattern rug on a loom

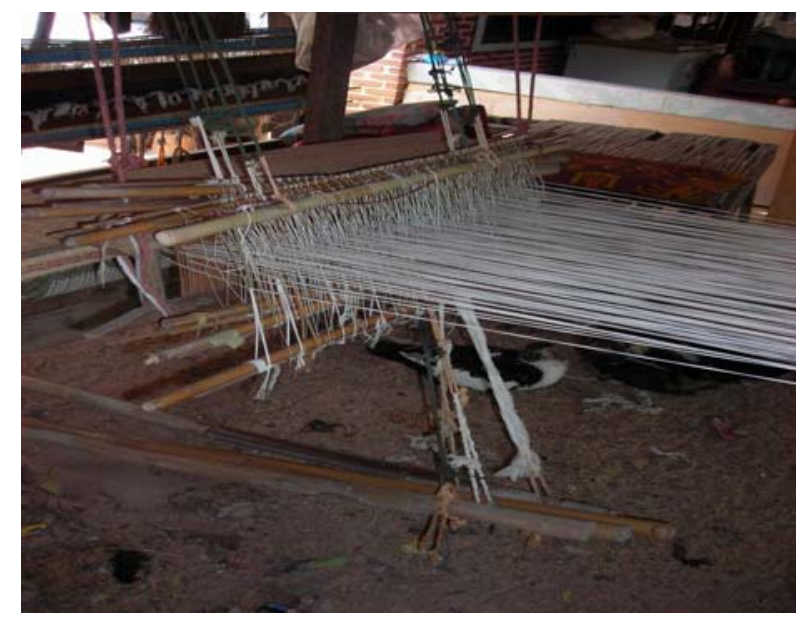

' 4 kao' loom with four foot pedals for rug weaving

Figure 5.3 ' 4 kao' loom with narrow reed and four foot pedals for rug making. 
Raweewan told the researcher that every member can weave plaid and striped cloth to meet the quota of 30 metres per month if the office does not give specific orders. Many members said that Prae Pun has a quota system in order to distribute equal income to every member. During the researcher's visit, members at every village were weaving plain cloth with handspun cotton for the monthly quota. Walai said that, currently, customers like hand-spun cotton cloth. At that time, Prae Pun had a large number of hand-spun plain cloth orders from a Japanese customer. Nowadays, however, only a few people know how to spin cotton. Thus, currently, Prae Pun prioritises skill at cotton spinning which is a traditional technique and requires much practice in order to be able to do well. Many members told the researcher that they can explain the steps of cotton spinning but cannot do it. They are busy with weaving and personal issues, so do not have not enough time to practise. Therefore, the committee approve that some members should do particular jobs, such as cotton spinning or dyeing, instead of weaving for Prae Pun. These members need to coordinate with weavers or through the office. As a result, senior villagers can join Prae Pun solely to make hand-spun cotton for the weaver members. Thus, Prae Pun can help to preserve traditional knowledge and provide opportunities to transfer this knowledge to the younger generation.

For weaving, each village group has set types of cloth for weaving on the basis of members' skills and a product variety policy. Every month, members weave the quota without an order. However, during the month, the manager might give specific orders to some particular village groups or members. The manager knows the specific skills of all the village groups and members. Also, each village group has its own way of distributing orders to members. At Huafai village, committee members know members' skills and can easily distribute orders. Fangnoi village has group meetings to find the members able to accept the orders. Villages can return particular orders to the office, if they cannot find members who are able to weave. It can be seen that there is a good connection between the office and village groups, and among members in each village group. This environment encourages sharing knowledge among each other. As members are rural villagers and work at home, they rarely possess knowledge of customers and marketing, so this knowledge is important for cloth design and new product creation. 
While talking with most members at villages, the researcher asked them to show her examples of cloth that they have woven. However, most of them do not have examples. They said that they do not keep them, but normally weave new designs:

We can design the cloth by ourselves for monthly weaving. We have to weave as orders when we have specific orders. We can mix coloured threads in our cloth as we like, so each member would make different designs. Normally, we use previous designs. We just change colours. However, we have designed new designs, such as dinosaur and forest. We have to draw the designs on paper, then we try weaving. If it isn't good, we will take it out (Raweewan).

The freedom in cloth design can influence members to create new products. From the researcher's own observations and stories from members, hand weaving is not just technical work. It requires inspiration and creative ideas. However, in order to design the cloth for Prae Pun, members need knowledge of customers for design and creativity: "We get samples from the office. The office staff members know customers' preferred designs. Customers might not like the designs that we choose. Customers like 'keelean' (qilin, an animal in fiction) the most. Sometime, NGO workers gave us copies of designs” (Onanong). Office staff members, especially sales staff members, and advisory committee members are vital sources for sharing customer knowledge for new product creation.

For old designs, generally, members obtain these from their villages. Neighbours in the same villages have a close relationship and feel free to visit each other and ask for help:

I look for beautiful designs in my village. Normally, we get Kit designs from old pillows... I borrow them from neighbours. We can ask from any villagers. I have to observe designs when I attend any merit ceremonies. Then, I borrow them when I do weaving. People are not possessive, because I will return the designs when I finish. (Malee). 
In Isan households, kit pillows (see appendix E) are commonly used, with. traditional pillows on traditional benches and in their glass cabinets. Generally, villagers receive them as gifts from ceremonies, and prepare them as gifts for guests and ceremonies as well. Compared with the traditional kit designs from text books, kit designs in the villages and local markets are simple. Prae Pun has more variety of kit designs than in the local markets, because Prae Pun has collected kit designs from study tours and text books, and adapted them into new designs. Kannika told the researcher that

Pare Pun has more kit designs than kit designs in Isan, because members have modified them them from mudmee designs by themselves. We don't have copyright, because they belong to Isan. They are Isan as well. Moreover, Isan people are poor, so they have a sharing mind and think of other people. About ownership, Isan is owner. The designs do not belong to only Phu Wieng, but they think of all Isan. Isan people are poor, so anyone who wants to weave, can weave. Isan is a sharing society because it is poor.

The above statements show that Prae Pun members focus on creativity in order to create a variety of products. Another point that can be seen from the above stories is that Prae Pun is influenced by Isan culture, which is considered a sharing society. Generally, senior members are designers who have long experience in weaving and are interested in weaving. They modify previous designs and create new ones while weaving. Members can borrow anyone's cloth as a sample and ask anyone to help them in design when they prepare thread at their weaving places:

Some members do not design by themselves. If they like my designs, they will take them. Designers have a role of design and anyone who like them can take them. We are not possessive, because our parents did like this. There is no school for teaching weaving. We have to learn by ourselves, or from parents and seniors. So, interested people have to observe from parents.

Many members told the researcher that one of Prae Pun's products, 'saifon' cloth, was created by weaving with leftover mudmee weft thread without paying attention to 
designs. Customers like saifon cloth, so now, members prepare mudmee weft for saifon weaving. Prae Pun members are creative and observant. They observe their results and capture weaving techniques. They are concerned with creating varieties of cloth to meet customer preferences in order to earn a regular income. Lada stated that

we used to make mudmee with the same designs for every lot, then, the office told us to find new designs in order to catch up customer preferences. We have to search our mother's designs .... The office tells us if we weave only one design, we cannot sell our cloth. We have to create new designs by modifying the previous designs.

Hand weaving can be viewed as local wisdom and indigenous knowledge that is derived through working with good practice, observation and creativity. In order to apply indigenous knowledge to a modern life style as shown in the products of Prae Pun, it requires adaptation in terms of particular knowledge, such as customer and marketing knowledge.

Most Prae Pun members like to work together with two to three members living in the same household group. At Nongthum village, two members set up their weaving looms at a small hut under the trees of one member's house (see figure 5.4). They are happy weaving and having conversations with each other and neighbours who visit them during weaving. At Suksomboon village, the researcher saw two members--one a senior member and another younger member--working together in order to help each other. One member wove while another member pun rod (prepared tube thread) and combed warp with starch liquid. Furthermore, most members prepare natural dyes together with group of three to four members in order to get the same colour for whole cloth batch.

These views reveal that Prae Pun is not only community-based, but it is also collaborative. The working environment of village groups involves socialisation and knowledge- and experience-sharing among members. Furthermore, knowledge transfer from senior members also occurs while working, because, generally, the 
groups are mixed between senior members and young members, or mothers and daughters.

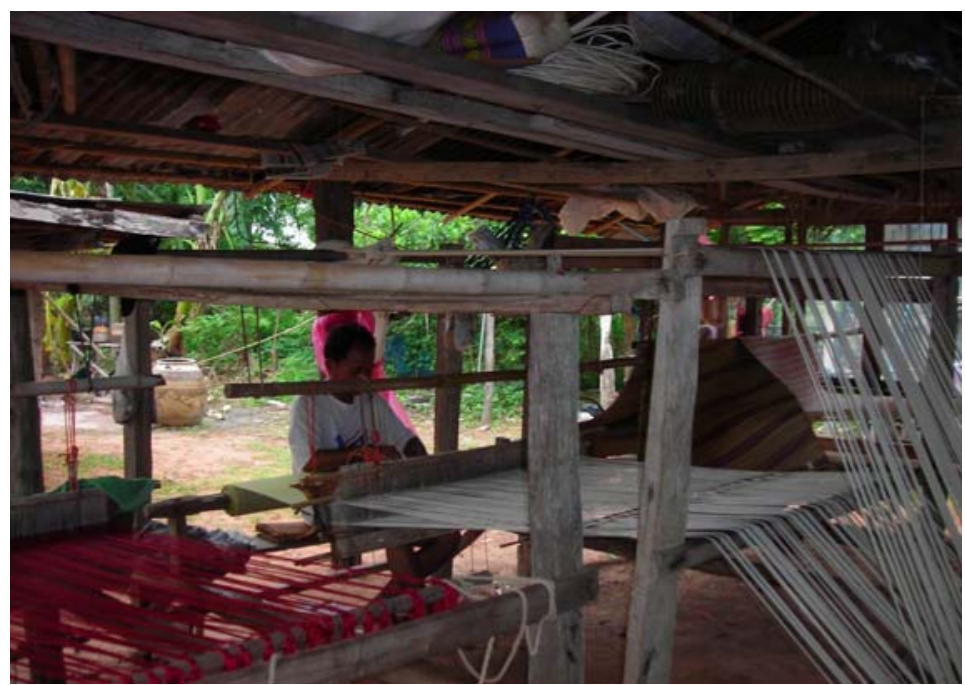

Figure 5.4 A weaving hut of two members at Nongthum village

Hand weaving is hard work. Weavers have to concentrate when they khondai (warp thread preparation) and have to sit a long time for weaving. Meanwhile, some processes require good eyesight to do, for instance, 'kleab kao'- preparing heddle shafts to make the kit design. Raweewan told the researcher that her eye sight is not good enough for kleab kao and kit designs, so she wants to teach young people to do that. Many senior members ask their daughters to help them in order to teach them at the same time. They need the young generation to replace them. Urai prepared warp thread as a sample, then, she asked her daughter to complete it, which she could do by following her mother's pattern... Urai's daughter understands the technique for preparing warp thread, but still needs to develop her own skills, especially design skill, in order to set up the pattern of the warp.

In addition to weaving, Urai normally asks her daughter to work on cloth checking and delivery, so her daughter can learn more about Prae Pun's activities:

Members deliver cloth to Rableam's house. My daughter can check identification marks and keep record about the marks, because cloth with a lot of identification marks would have low prices... I may go 
for cloth delivery for sometime when my daughter is busy. I do not teach her cloth checking. She does not work alone. She works with two other committee members (Urai).

Thus, Urai's daughter does not learn only hand weaving, but also Prae Pun's working systems and culture. Other new members as well have opportunities to learn about the Prae Pun working systems and culture through their work processes. At the beginning of each month, cloth is delivered to the office by group representatives. The Nongthum village group has a roster for monthly cloth delivery, in order to give every member the opportunity to visit the office. Napaporn told the researcher that "we set cloth delivery rosters of two members per month in order to give them opportunities to view their own property and cloth from other village groups".

Cloth has to be checked for quality by village committee members before it is sent to the office. Walai stated that "the quality control committee has three members. Two members check cloth and another member records. The document has two copies. One copy is for us and another copy is for the office in order to check paying”. At the office, office staff members check all the cloth again and decide the levels of quality and prices. Kamolwan said that

some members do not accept the decision of village committee. They ask us to take their cloth to the manager to make decision, so we have to do that. The manager will give them the results of checking in document that shows the level of cloth quality. Then, they will accept the manager's decision.

The office will pay full price in cash for cloth that meet the standard, lower prices for cloth that does not meet the standard, or accept poor quality cloth on consignment. Moreover, the manager might ask particular members to stop weaving, if their cloth is not good enough for selling. Each village group has to put aside two to three per cent of the price of cloth sold to cover delivery costs and group activities.

In a nutshell, members do not have only hand weaving and natural dye knowledge, but also have operational experience. Working also allows them to absorb Prae Pun 
culture, especially through ownership of the CE and a sense of belonging to the same group, even though Prae Pun comprises seven village groups. The sense of ownership influenced them to contribute to Prae Pun - to work hard and continue learning and searching for knowledge-while the community spirit expands members' sense of sharing not only for their own village group, but also for all seven village groups.

\subsubsection{Learning how to weave}

I started weaving when I was 15 years old... I started weaving with white plain cloth. I wove after school when my mother paused weaving, because I liked weaving. When I was 17 years old, I started weaving kit because my mother asked me to prepare kit for my brothers' upasampada ceremonies. I have four brothers. Upasampada ceremonies need kit pillows and kit mattress. I was very happy when I could weave kit. I did not take long time to learn... I could not weave when my mother was staying with me, but I could weave when I was alone. I wove it by following her instruction (Kamolwan).

In Thai society, daughters are supposed to help their mothers in taking care of family, cooking and tidying the home. In Isan society, daughters are also asked to help their mothers with weaving for domestic consumption and ceremonies. Previously, Isan societies had to grow cotton, engage in sericulture and prepare cloth for domestic consumption and ceremonies instead of buying it from the markets: "Our parent taught us weaving because we did not have much money to buy cloth. Seniors did sericulture and silk bleaching by themselves and taught us to do those things (Lada)”. So, generally, Isan women learn how to weave from their mothers or female relatives. They can weave the same types of cloth as their parents. Suwannee said that

people who are interested in weaving can weave when they see their mother weaving and weave as their mother are doing. People who cannot weave are people who are not interested in weaving and have not seen their mothers weaving. People who are interested are be able to weave after only one loom of practice. 
During her visit, the researcher saw a few young women aged from 15 to their early 20s weaving. As many members said, nowadays, young people go to school and then work in Bangkok after finishing school. Generally, they would come back to their home villages when they were married in order to settle down, do farm work and take care of children. Many members who have daughters teach them weaving in order to help them make cloth for Prae Pun rather than for their own consumption. Suwannee's daughter was weaving while Suwannee took care of her grandson. Suwannee told the researcher that "I taught my daughter weaving. Now, she can weave for me when I go to a paddy field. I taught her two to three years ago when she came back to deliver a baby... I told her how to weave and she observed my weaving. Now, she has become a good weaver”. Malee said that hand weaving is not as hard as studying at school. Hand weaving is not difficult, because they have watched their mothers and other villagers weaving since they were young. Some members' daughters told the researcher that hand weaving is familiar here. They see their mothers and villagers weaving and understand how to weave. They just need practice in terms of direct experience and building their skills. It can be seen that hand weaving is learnt through traditional methods through observation and doing, with supervision from mothers or relatives.

Even though villagers are already good weavers, they have to be trained when they become Prae Pun members. Cloth for selling is different from cloth for domestic consumption. Kamolwan said,

we have to give advice to new members. Then, they can do by themselves because they used to weave. Like working in Bangkok, weaving for selling has to weave to meet customer preferences. When I went to work for a laundry company in Bangkok, they asked me whether I could do ironing. I answered 'yes', but they told me that I could not do it, because their ironing was different from my ironing.

The researcher saw that the cloth that members weave is different from the cloth used in villages. Members weave place mats, table sheets, bed sheets, rugs, etc. All Isan rural houses that the researcher visited do not have these kinds of cloth in use. Isan rural houses are simple. They mainly do most activities on the floor and traditional 
benches with big grass mats. Furthermore, they have different preferences from city people. They prefer bright and contrasting colours, whereas city people prefer matching colours. The cloth traditionally worn by villagers and seen in the local markets has very bright colours and mixes contrasting colours together very boldly. Meanwhile, Prae Pun products are earth toned and mix only the same shades of colours. Members have to learn colour matching and customer preferences:

\begin{abstract}
About the same shade colour design, the NWD training taught us master colours and colour matching. For instance, if we use a white insert in cloth that is not white, the result would be cloth with contrast colours. We observe this for ourselves what the trainer told us was correct (Parichat).
\end{abstract}

Even though members attended training on colour matching, Kannika told the researcher that they took very long time to change their design and colour ideas. Parichat said that they would accept new knowledge once they realised its truth from their work. As a result, true knowledge used by members is practical knowledge that members obtain through working and direct experience.

New members have to learn how to weave for Prae Pun. As stated previously, Prae Pun has quality standards and new members receive orientation and advice from village committee members in order to help them weave cloth to meet these standards. Recently, new members have had to visit current members in their villages instead of study tours to other places. Compared to when it began, Prae Pun has few study tours and training programmes, because, currently, Prae Pun has many experts of its own and has to use its profits to conduct such programmes. The study tours at the beginning phase of the CE's development were supported by NGOs, especially the Handicraft Centre for Northeast Women's Development (NWD). Pitsamai said that, when she became a member, she just visited members in her village and other Prae Pun villages, and attended only in-house training programmes. Then, she had to practise by herself and ask members in her village for help:

Experienced members often visit new members. They have to help us when we become members. They came to tell me about my weaving 
results and how to fix any problems. If members in this village cannot fix the problems, we will ask office staff members or Mae Som who has been a member for long time. We will pick up her to fix the problems at our place (Kunya).

At the beginning, Prae Pun also conducted many training programmes for members and committee members. Most members who joined at the beginning stage told the researcher that they attended many training programmes and study tours organised by NWD.

At beginning, Mae $\mathrm{Al}$ gave a training programme at our village hall. She taught us natural dyes... At that time, many NGO workers - Arjan $\mathrm{Pu}$, Sao Noi, and etc. came to our village every month (Kamolwan).

It can be seen that currently, Prae Pun has a number of skilful members. Knowledge capture of new members is focused on internal knowledge capture through working with supervision from skilful members. On the one hand, initial knowledge capture of Prae Pun focused on study tours and training programmes. Now, however, the training programmes focus on internal knowledge capture for which skilful members are the resource persons.

Most initial training programmes were focused on cloth design and natural dyes that would make Prae Pun products attractive in the markets. Because the members are rural farmers, at the initial stage, Prae Pun lacked customer and marketing knowledge. Lada said, "I was trained in many things. I attended a training activity on mudmee design and design selection. For example cloth with big designs is suitable for slim people. It was very hard to learn about the new designs, and I often had a headache”. Also, Prae Pun focused more on weaving techniques and cloth quality in order to make varieties of good quality products:

Previously, our village did not weave with the ' 3 kao' technique. Old members got this technique from a study tour.... Then, we began weaving with that technique. Because I already knew how to weave, I needed to practise the new technique for only two to three days. I just 
learned how to use foot pedals and after, we practised for a while we would know the right or wrong steps.

Generally, common cloth is made from a loom with '2 kao' or two heddle shafts that are controlled by two foot pedals. In order to make float patterns on cloth, it requires more than two heddle shafts with weavers who have advanced skills at weaving. Therefore, skilful members at '3-kao' and '4-kao' make varieties of cloth patterns for Prae Pun in order to obtain good markets, and Prae Pun trains new members to weave with '3-kao' and '4-kao' techniques.

Most long-term members have been to many places to learn and observe. However, most programmes cannot accommodate all members at one time, and most members are not available to attend. For example Raweewan indicated that she did not visit as many places as other long-germ members because she was busy with her children. Normally, only two to three members per village group can attend a given programme, and they would train other members when they return from the programme. Raweewan told the researcher,

The members who went to Roi-et province trained us how to use 'tong leang' technique that keeps kit designs at kao. Without tong leang technique we have to make kit designs every time when we weave kit.

At the beginning, Prae Pun focused on training the trainers. Thus, NWD tried to train key members from each village group to be resource persons who could help members in their villages: "We attended a training programme in cloth quality control with Arjan Ung in Khon Kaen. He taught us the cloth standards for checking the quality of cloth. Then, we had a workshop at our showroom and informed our members of the standards and implemented the quality control policies” (Walai). After NWD withdrew, Prae Pun has used its profits to fund education programmes. Most of the programmes are internal programmes and involve committee members and key people from each village group:

Even though we do not have NWD, we have organised study tours and training activities by ourselves. Now, we are studying indigo dyeing. 
We do not do pure indigo, but we would mix indigo to our colours. We sent two members from each village to study indigo dyeing in Sakolnakorn province, and now, we are doing experimentation at our villages. Now, we can do indigo dyeing.

It can be seen from the stories that traditional knowledge is learned through traditional methods: doing, observing and advising by skilful people rather than formal education and activities. As hand weaving is women's work, members mainly learn hand weaving from their mothers or female relatives, and practise by themselves. In order to utilise traditional knowledge for modern products requires modification using creativity and particular knowledge, such as customer and marketing knowledge. Prae Pun obtains these kinds of knowledge through many activities including training activities, study tours and self-learning through working and observing. In fact, Prae Pun has many training activities and study tours to develop further knowledge and train resource persons in natural dyes and business management. These stories will be told in the following sections.

\subsubsection{Natural dyes}

At Urai's house in Haufai village, there were many cotton bundles hanging on a bamboo stem beside her house, and Urai sat on a traditional bench at the ground floor of her house. She looked tired and her hands were stained with natural dyes. She had just finished making grey cotton dye from 'boa' and 'makleo'. Some members told the researcher that cotton dyeing was heavy work, because cotton is heavy when it is soaked, but their daughters did not want to help them. The daughters do not like natural dyes because they make their hands look unattractive. Indeed, natural dyeing is hard work because the cotton needs to be dyed many times in order to produce a good colour.

Makleo is a well known traditional natural dye. Many years ago, farmers used makleo to dye working cloth, fishing nets and silk. Raweewan said that "generally, makleo is used to make the colour black for silk". And Walai stated that "her grandmother said cloth dyed with makleo is comfortable to wear when working outdoors because it can help us not feel hot”. Currently, Prae Pun used makleo to make the colour black and other colours become dark 
At the beginning, we didn’t know that small mango bark can give dark green colour. They told us only small mango bark can make green colour, but we need dark green. Then, we tried a double dyeing technique by using makleo. So, we got the dark green as we want. In fact, we heard that makleo could make dark colours from members. We always tell each other when we know any techniques (Raweewan).

Members know well how to use makleo for natural dyes because they saw senior villagers using it. Currently, natural dyes are old fashioned. Most villagers use chemical dyes because they give bright colours and have a long life. Many senior villagers retain traditional knowledge and techniques and share these with Prae Pun members. Members obtain basic natural dyeing skills from NGO workers. Then, they develop their dye making skills further through observation and practice:

I learnt natural dyes from the staff of the handicraft centre. They told us the sorts of barks that can give colours. Then, we did experimentation by ourselves because the staff didn't know much about trees in our areas. We found that each bark can give more than one colour. If we added alum, it would give one colour. If we added lime, it would give another colour...We have to hew bark in order to check a colour. It gives the same colour as its wood oil colour. For instance, it will give red colour, if its wood oil is red (Raweewan).

It can be seen from Raweewan's statements that makleo is indigenous knowledge which senior villagers know how to use and transfer to Prae Pun members. Prae Pun has to apply indigenous knowledge to suit its circumstances. For instance, members apply makleo to make varieties of natural colours rather than black. However, not all village groups can use makleo, because Raweewan said that makleo can only be found in paddy fields that have forest areas. However, some villages are low flat areas without a forest area. Furthermore, they can collect makleo only in summer, and then. preserve it for the whole year. Not only the use of makleo relies on nature, but also other materials, such as bark. Most members commented that the bark from different seasons gives varying colours. 
All members whom the researcher talked with told the researcher that natural dyes depend on the nature and environment of each area. The seven villages would not end up with the same colour when they made natural dyes with the same sorts of materials and methods. Napaporn found that she could not make the beautiful colours she made when she attended a training course at another village, because the water from the wells in her village is different from the water in other villages. Water from deep wells that have dark or rusty water can make dark natural dyes. Water in her village is clear, so it gives light colours. She has to dye many times in order to make dark colours. Each village group dyes using specific colours with particular techniques. Members in villages that do not have rusty water from deep wells have to prepare rusty water by themselves. They soak metal in a big jar in order to get a dark colour. Meanwhile, members at Huafai village make dark colours by using makleo or mud:

We have to take mud from the field to make at home and can soak only a few minutes. My neighbour just did this technique when she made a red colour. Other villages also use this technique. Mud can help to make darker colour. Sawai just did 'samol' dyeing with mud last month.

Natural dyes vary from place to place and also from season to season. They cannot make the same colours every time. They have to tell customers that they may not get the same colours as in the catalogue. This is another reason for members to work together as a small group when they have orders, so that they can get the same colours for a whole batch. It can be seen that working with natural dyes, members have to observe and apply knowledge based on a combination of village nature, and customer and market situation.

Most of Prae Pun's products are brown toned colours and become pale very soon after use. Many members told the researcher that most barks give brown colour. Recently, they have studied indigo and 'mug chatree' or annatto fruit dyes in order to get blue and orange colours. Indigo dye is well known in Sakolnakorn province. Traditionally, "Phu Thai" women—one tribe in Isan-wear blouses and simple skirts dyed with indigo. So, hand weaving groups in Sakolnakorn are good at producing indigo dye; 
Many years ago, this village had villagers who made indigo dye. But recently, no villager in our village makes indigo dye. We went to study from Sakolnakorn province. We still cannot make it as well as them. This might be because they did not tell us all techniques... Nowadays, there is only one villager alive who used to make it. She tried to do it but she was unsuccessful....(Kamolwan)

In fact, indigo dye is a traditional dye in Isan areas including Khon Kaen province, but it is out of fashion. Thus, villagers lost their skills at indigo dye. Prae Pun has to capture the knowledge from other sources and develop their skills by practice. Prae Pun has realised the necessity of the varieties of natural dyes for its products. Prae Pun does not only capture the knowledge of natural dyes from other sources, but they also search for the knowledge themselves through experimentation and practice.

At Prae Pun, committee members conducted a natural dyeing workshop last summer. Many members commented that the workshop was designed to search for new colours, materials and techniques:

We had a natural dye training programme at Nongyanlan village on $17-18^{\text {th }}$ March... We focus on 'mug chatree' dyeing for a yellow colour. Each village had to respond on one colour. We were excited because we had not had a training activity for a long time. I was responsible for the black colour from makleo but I did not make it, because I did not have makleo at that time.

At a committee meeting, the committee members discussed the results of the natural dye experimentation at the Nongyanlan village workshop. They compared cloths that were dyed with different substances and tested them for exposure to the sun. The researcher was very surprised. There were many more beautiful colours than at the showroom. For instance, there are bright green, yellow, orange, and pink. These colours will be available at the showroom soon, because the committee asks each village group to continue to study and record experimentation in order to share such knowledge with other members. Thus, any techniques that can give proper colours and do not fade out in a particular will be applied to their work processes. It can be 
seen from the stories that Prae Pun members are experimenters. They search for and test knowledge through experimentation. The experimentation has not only been conducted as formal or group experimentation, but also as informal experimentation by individual members:

If we did not know any colour, we would try with less cotton... we try when we get new leaves. If we get a good colour, other members would ask us how to make it. We always try with barks that we see have beautiful colours. We have tried to use leaves and fruits instead of bark because we worry the trees will die. However, if we use bark, we do not cut it deeply and cover the cut with mud. Our seniors did like this and told us that helped the trees to develop new bark (Pitsamai).

Compared with other regions, Isan is dry and has a few trees. There is a wider view of the paddy field when standing outside a village, because they are a few big trees to block visibility. As a result, Prae Pun has encountered a materials shortage for natural dyes. Members have to search for new sources of colours, such as leaves and fruits:

At the beginning, we didn't know that fruits could be used for dyeing until we faced problem of bark shortage. So, bark can be used for dyeing, fruits and leaves can be used as well. Then, we did experimentation. We found that mango leaves and 'huklwan' leaves give bright green colour, makleo gives yellow colour (Walai).

Members observe the colours of bark and plants all the time. Malai told the researcher that she knew that coconut husk could give colour because she saw a colour on her knife when she removed the coconut husk. After that she used the coconut husk with rusty water for dyeing. Members have had the idea that any parts of the tree could give the same colours as its bark. They have the characteristics of researchersobserve and try. As a result, most Prae Pun knowledge comes from observations, and a trial and error method. However, members have limits in terms of documentation. Pitsamai said she tried to use many materials for natural dyeing, but she could not remember any of the recipes for the beautiful colours she had made. 
Prae Pun should have many opportunities to create new beautiful colours through experimentation, and apply to work processes, because members focus on searching and testing the use of substitute materials for natural dyes. Thus, experimentation can be referred to as knowledge creation and testing for Prae Pun.

\subsection{Being Committee Members}

On a committee meeting day, committee members from each village group came to the office before the meeting started. They chatted with each other in a very friendly manner at a show room and some of them helped staff members prepare lunch at a kitchen which is located next to the showroom. In some corners of the showroom, young committee members read magazines and explored products on the shelf. The meeting took place at the showroom. All committee members, staff members and an advisory committee member sat on the floor in an informal manner. They could stretch their legs forward and eat snacks during the meeting (see figure 5.5). Committee members, advisory committee members and staff members were considered as part of the board committee. They had very open discussions with arguments and opinions. Kannika—an advisory committee member—said,

We have talked openly in the committee meeting. I have given them information, they have to make decisions. After that they do not gossip. They have been trained in that.... We have a lot of discussions. Some times we were upset. The committee members have improved their management skills a lot because they can express their ideas and make decisions by themselves. We have to encourage them when they do well. 


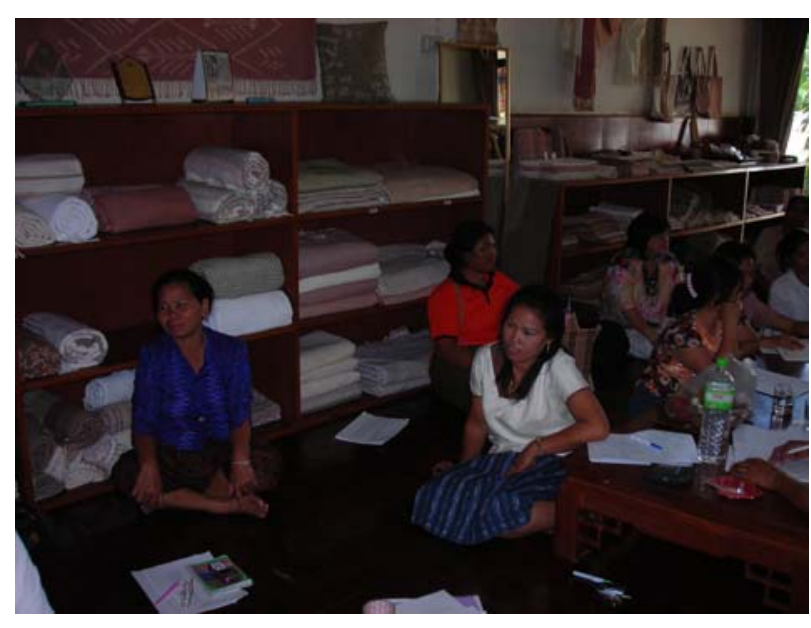

All committee members and staff members sit on the floor at the showroom for a committee meeting.

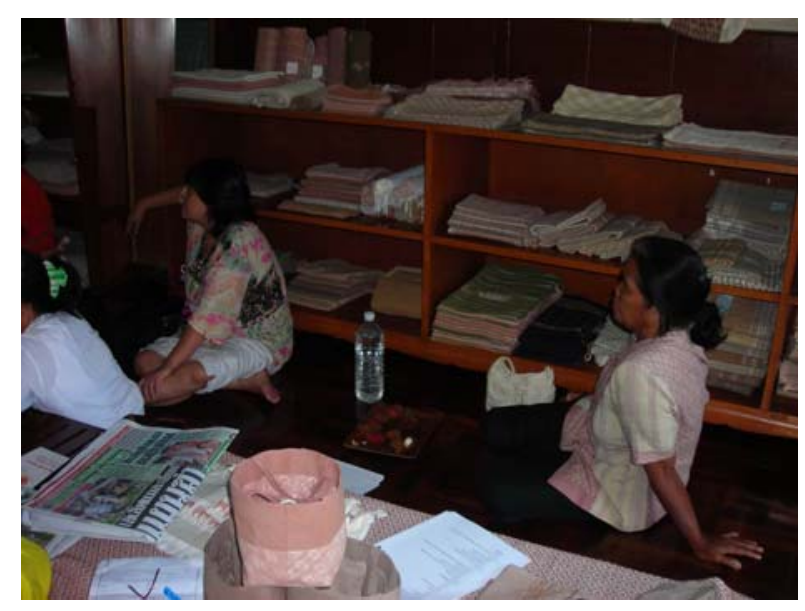

Committee members can sit in a relaxed position and eat while having a meeting.

Figure 5.5 A committee meeting environment

However, young committee members kept quiet at the meeting. They listened to the other committee members and discussed with colleagues who sat near them rather than speaking out to the meeting. They gave opinions only when the chairperson or advisory committee member asked them. Being a committee member is a pressure for new committee members. They are shy and not confident about expressing their opinions. This is because the broad committee comprises senior committee members, who have a lot of experience in weaving and enterprise management, and advisory committee members, who are NGO workers. New committee members feel that they are not as good as long-term committee members. Meanwhile, senior committee members are confident because they have been trained in many issues, including group and business management, and gender issues.

It can be seen that new committee members have not yet assimilated Prae Pun culture. They still act like general rural Isan villagers that have to be kreng jai or show consideration to seniors, and on nom thom ton, or lower oneself to other people. Meanwhile, long-term committee members can express their opinions through the committee meeting. This is because they have the feeling of being equal with responsibility for managing Prae Pun. Thus, the training programmes for the committee members do not provide only management capacity building, but also Prae Pun acculturation. Kannika stated that 
We conducted gender development training programmes and study tours... Villagers should be developed with critical thinking... They can do so because they have the opportunity to do so. We try to create a sense of ownership for them. They have to practise, because they are owners and do not want anyone to take over... They practise, then, they have skills... They can do this because they have self-confidence and ownership.

Surprisingly, the board committee has no election system to recruit new committee members. They become committee members by nomination. Each village group has to nominate two committee members. Then, they can work without setting a term of service. For instance, the Prae Pun president has been in that role for over 10 years and committee members still want her to continue. Some senior committee members who want to resign have to nominate appropriate members who are good at weaving and able to travel and do document work. Committee members have to attend committee meetings, which normally take place at the office, and study tours as part of their capability building programme. Napaporn said

committee members have to take responsibility. Sometime, we had to come back home at night by foot. My husband was sick, so I had to resign. The board committee asked me to find any member to replace my position, then, the committee would approve my resignation. I got my niece to replace me. In fact, we want young members to be committee members, because senior members get difficulties and we want them to learn from us. My niece is available to work and good at documentation.

Committee members have to be available to travel away from their villages for many activities, such as committee meetings, cloth delivery, guest welcoming and study tours. Indeed, committee members should also be good at problem-solving and finding, communication, documentation, and have good ethics. Napaporn said, "committee members have to discuss in order to find solutions for any problems, such as weaving and management problems, and to create new activities. The meetings may take two days. We have to stay overnight at the office”. The committee members 
present an image of being good at management, hand weaving and good ethics. Thus, being committee members is considered an honour which can be referred to as an incentive system to work hard and continue study to be good at hand weaving. Committee members are dedicated members, and work with honesty and justice. They have to work for the benefit of members. This can be seen from the following stories:

The committee members have to work on quality control without thinking of their relationship with members. They cannot be biased to their relatives. We are very friendly with each other and are relatives with each other when we do not inspect cloth. The committee members have to work in a impartial way to judge members' cloth even if their relatives' cloth is not good... committee members have to dedicate themselves to attend meetings and study trips outside our village (Walai).

Committee members have some limits. As they are rural farmers, they lack skills in business management and documentation. They are also unfamiliar with city lifestyles, which is a necessary factor for creating marketing strategies and designing products because most customers are city people. Members have visited many places on study tours in order to widen their world view and enlarge their experience and observation.

At a committee meeting, the committee members reflected on the last trip to Chiang Mai province and planned for the next trip. They went to learn about other handicraft groups in Chiang Mai, which is well known for handicraft businesses, in order to get some ideas to expand Prae Pun's businesses. For the next trip, they planned to visit many well known hand weaving groups in Isan in order to explore their working, products and cloth designs. However, they need to verify and apply the experience from the trips, because Prae Pun has a different situation. Raweewan stated that

The Chiang Mai trip was organised for committee members with the objective of looking for alternative businesses in order to expand our businesses. We found that we could not do as them because our areas were not the same as their areas. It would be better to improve our 
weaving skills. They do bamboo crafts. We may exchange products for selling at our shop.

Another duty that new committee members worry about is the supporting programme. Committee members have to visit members in their villages in order to follow up their work and suggest weaving techniques in order to help them meet the cloth standards. Walai stated that "village committee members have to call members for a meeting in order to inform members to improve their weaving. In case the members cannot improve their skills, the committee members have to help them in weaving processes”. Thus, the committee members have high social connection and interaction with members. The committee support programme is one effective channel for knowledge sharing and transfer among members, especially from skilful members to new members.

In fact, Prae Pun does not want committee members who are only good at hand weaving and natural dyes, but who are also good at documentation. This is because committee members have to do the operational work at the village group level instead of the office staff members. Prae Pun needs to keep records of membership, production and cloth delivery and the knowledge derived from many activities. As Walai's story showed, the cloth standards committee has to prepare documents about their cloth inspection for the office. As well as operational documentation, the committee members are key people for recording knowledge acquired from other places and activities, because they have more opportunities than members to visit other places. Most committee members whom the researcher talked with have notebooks in their hand bags, containing cloth designs or weaving techniques that they got from study tours. However, some committee members could not find their notebooks, and many do not often use them, because they can recall their experience for working.. These notebooks can be referred to as sources of knowledge for Prae Pun. This knowledge would be lost, if particular committee members are not interested in utilising the knowledge captured and recorded for work processes.

The researcher observed that most early members and committee members have a strong feeling of ownership because they have been members since Prae Pun had nothing-no money, no office, and few techniques for making good quality cloth. 
Currently, however, Prae Pun has a building, money and knowledge about making good quality cloth. As explained previously, they are proud to be Prae Pun members, because they have developed everything by themselves through hard work. Recently, they have been thinking of how to pass Prae Pun on to the younger generation. Most members told the researcher that they want their children to replace them. Many senior members said that they are tired and want to stop weaving but are keen for Prae Pun to continue. Thus, most senior committee members and senior members pay attention to transferring knowledge to young members, especially their daughters. They focus on both technical knowledge and Prae Pun culture and values. It can be seen from the stories that senior members are proud, not only because Prae Pun has high quality and unique cloth, but also because Prae Pun, the enterprise, and its culture and values, have been established and developed by the members themselves.

\subsection{Office work}

\subsubsection{At the office}

Along the Chathapadunk Road, the Prae Pun office is different from every other office in the area. The Prae Pun showroom and office is a four-storey building in Khon Kaen city. Prae Pun has decorated the exterior of the building with a traditional Isan roof and potted plants (see figure 5.6). The Prae Pun office gives a feeling of home and Isan culture, instead of a commercial townhouse like other units, for people who pass through this area. This atmosphere involves the office staff members, members and customers feeling comfortable as if visiting a home rather than an office. Many members told the researcher that they always stop to relax at the office when they come to Khon Kaen city. Thus, the informal environment of the office allows office staff members to feel free to talk and have discussions with managers, committee members, advisory committee members and each other.

The ground floor is a showroom, the first floor is an office, the second and the third floors are stock rooms and staff members' bedrooms. The showroom is designed in the traditional Thai style with a wooden floor and short leg traditional desks. Staff members and customers have to take off their shoes before entering the showroom and sit on the floor. The researcher saw a sales staff member sitting on the floor and stretching her legs forward in order to make cotton twist on her legs. The first floor is a small office which has an open space style. Each staff member has a small desk 
located next to one another without partitions. However, staff members prefer to work in the showroom, because they can sit in a relaxed position and talk with each other and the customers while working. Figure 5.7 shows the Prae Pun office and showroom.

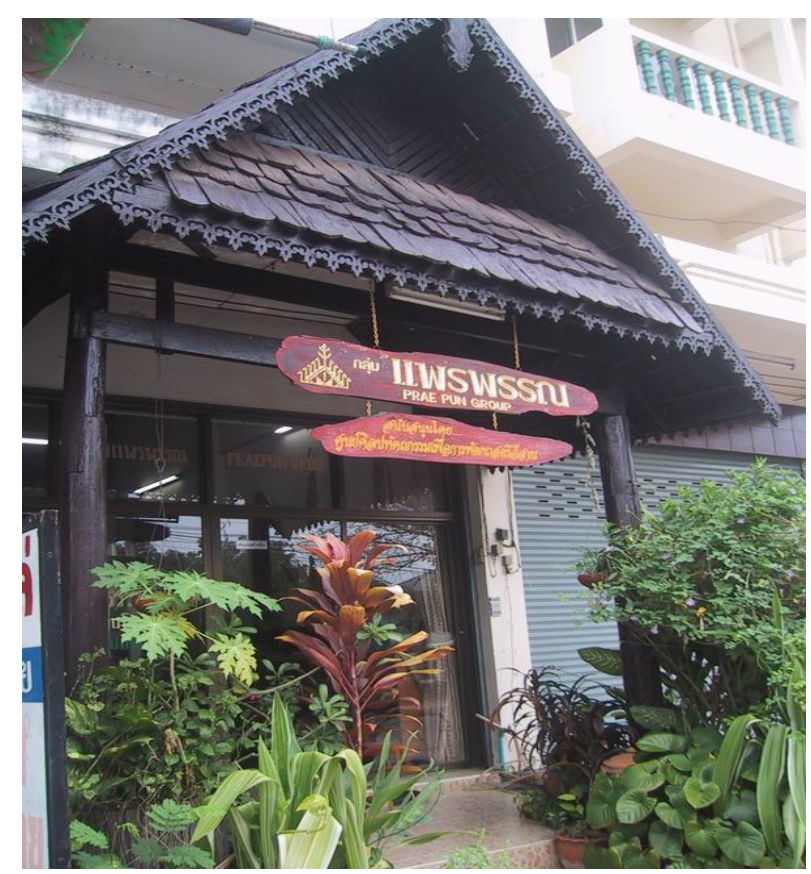

Figure 5.6 Prae Pun office and showroom: from the external side
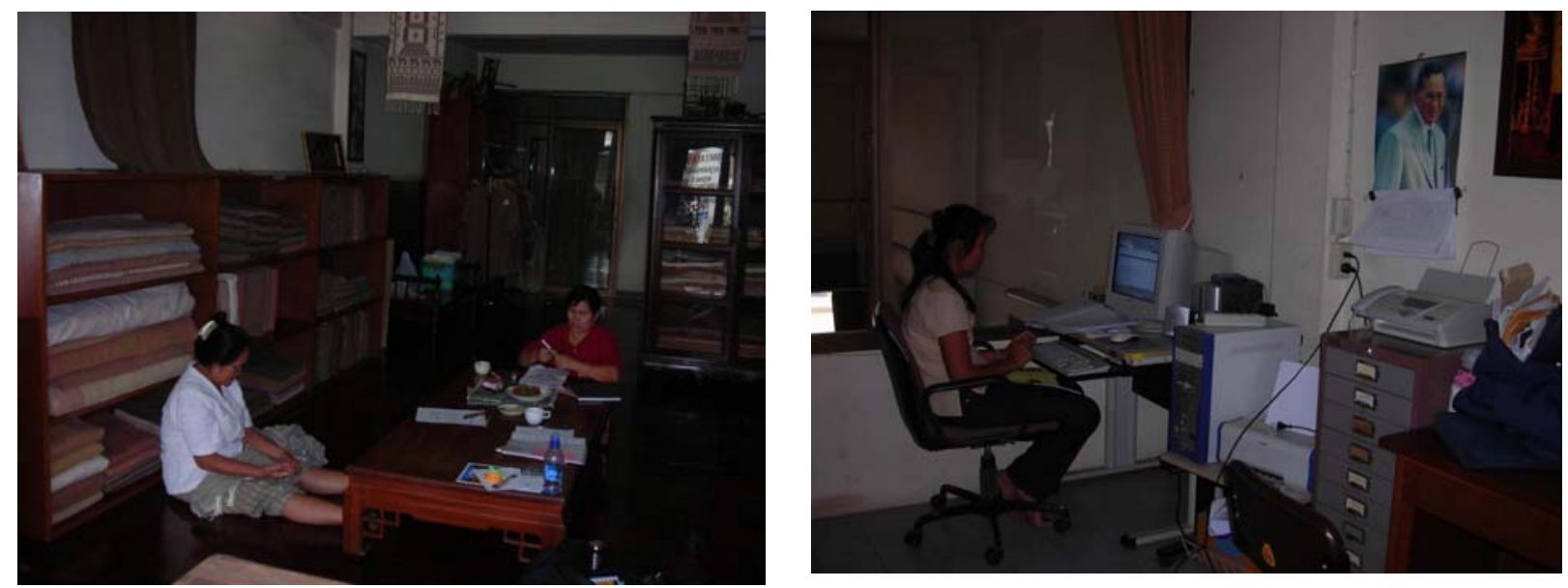

The showroom is in a traditional style where staff The small office on the first floor. members and customers have to sit on the floor.

Figure 5.7 Internal Prae Pun office and showroom. 
Office staff members like to work in the showroom rather than in the office on the first floor, except when they need to work on the computer in the office. The researcher saw them having discussions with other staff members while they are working. They pause in their work to help a sales staff member when customers come to buy their products, especially for wholesale purchases. They know each others' work and can help each other, except for specific tasks - accounting and financing, welfare and documentation. In fact, every staff member can take care of customers and take orders. Furthermore, they have to take part in selling at handicraft fairs (see section 5.2.2). It can be seen that the office work involves job sharing and socialisation amongst office staff members:

My duties include selling at the showroom and handicraft fairs which need two people, and shipping cloth at a post office. In fact, the shipping is not my duty but we help each other when we have a lot of work. I would do cotton twist when I do not have any work... Other staff members can take orders as well because we see each other work and we were trained together but I was not trained in accounting... (Penchun).

The office also has bedrooms for office staff members. Two staff members stay overnight at the office because their homes are located far from Khon Kaen city and sales staff have to stay at the office in order to take care of the showroom. Two of them have a rotation for one day off to go back home, because the showroom is open seven days:

Office staff members are comfortable in the work environment because they have only one boss-a manager who is flexible. Even though our shop is open seven days a week, the office staff members can take a day off when they are busy with their family. This is because Mae Nee sets a day-off rotation system (Kannika).

The office has many factors that encourage sharing among staff members as well as with customers. The most important thing that makes the Prae Pun office atmosphere informal and friendly is its working style. All the staff members are from Prae Pun 
villages. They are members and daughters of members. They work like family members rather than employees, or boss and subordinates. Office staff members call each other the same as villagers call each other. They use the word 'Mae' or mother in front of the name of women who have children:

We want members' daughters to work for Prae Pun because they would feel like owners and love Prae Pun, because we work together as a family. We do not have a boss and subordinates. We help each other. We can replace each other if someone is not available. We do not think that we are employees. We stay here as family (Penchun).

Staff members feel free to talk with the manager and with each other. They always have breakfast or lunch together in the office's kitchen or the showroom. During the researcher's visit, Penchun brought bamboo shoots from her village to cook Isan curry at the office. She got up very early like villagers in rural areas in order to cook and clean the office and the showroom. In the evening, she closes the showroom when it is dark after the other staff members go back home at around 5pm.

Even though the office work is informal, office staff members work and study hard to develop their abilities. The office is quite a different environment from the village environment. Office staff members have been trained and practise in many areas. Malee said that

I graduated only in grade four and I had not done any study after school. I could not use a calculator. NWD staff members taught me how to talk with customers. I knew only how to talk with villagers which was different. I had to learn everything including how to answer a telephone. At the beginning, I did not know how to talk on the telephone. I could not sleep when the telephones rang because I was afraid and I did not answer them. They rang and did not stop. I sweated when I answered them because I did not know what I was talking about. Then, it was better. 
Many members told the researcher that NWD conducted many training programmes, including accounting, stock control, selling and ordering, before withdrawing its support: "NWD staff members taught me how to maintain stock. I have to check stock on the $28^{\text {th }}$ or $29^{\text {th }}$ of each month. I have to subtract the amount sold from the stock. We had practised for two months" (Penchun). After NWD withdrew, Prae Pun has had to train new staff members itself through its working processes and an internship programme. Wijittra said that "I started working in 2002. I was in a probation period for three months. Office staff members taught me much about documentation work and types of cloth. At the beginning, I worked in the selling section and Mae Nee also trained me in accounting, including receivable accounts and income statements”. Penchun said that "we accept any members to be interns every year. We want members to learn office work and marketing work. We will accept them to be a staff member if they can work. As interns, we pay them 2000 baht/month”.

In a nutshell, most of Prae Pun's business management systems were designed by NWD and transferred to office staff members and committee members at the beginning. It can be seen that office staff members mainly develop Prae Pun knowledge and operational and management skills through working and internship. Recently, office staff members are more confident and know how to deal with customers, suppliers and fair trade organisations (FTOs). They understand more about the city and modern life styles. The researcher visited one staff member's home which is located in a rural village. Compared with the researcher's host mother's home, her home was tidy and she welcomed the researcher with a beautiful, nice glass and a jug. This means that she now has more understanding of the customer's viewpoint, which may help Prae Pun to catch up on customer preferences.

\subsubsection{At handicraft fairs}

Prae Pun sells its products at many handicraft fairs in Bangkok and other provinces, but has one regular handicraft fair-Thai Craft. Thai Craft is a fair trade organisation conducting handicraft fairs once a month in Bangkok, and tours for customers to visit handicraft producers. At the Thai Craft fair, two office staff members set up their booth and sell the products at a well known hotel in Bangkok. They did not need to explain much about products because most customers are old customers and familiar with Thai Craft fairs and products. However, Thai Craft provided a foreign volunteer 
to help Prae Pun explain products to foreign customers. Furthermore, Thai Craft provided posters and leaflets in English and Japanese for customers. Some customers were handicraft business men who contacted Prae Pun for specific wholesale orders.

At another time of the researcher's visit, the Thai Craft fair focused on Isan culture. It had an Isan handicraft exhibition and Isan cultural shows. Prae Pun took some natural dyeing materials, such as bark, makleo and klang (lac), and a loom to the exhibition. On that day, two members had to help office staff members to demonstrate kit weaving and cotton spinning. Some customers were interested in kit weaving. They came to observe for a long time and some customers wanted to buy dyed cotton for their artwork. Thus, selling at handicraft fairs and exhibitions allow Prae Pun to obtain information about customers, especially their preferences for Prae Pun products. As mentioned previously, this kind of knowledge is useful for the product creation of Prae Pun.

\subsection{Advisory committee members}

The researcher visited Prae Pun's villages with one advisory committee member who was a senior NGO worker. She talked with many villagers, both Prae Pun members and others, in order to catch up with their lives and work. She talked with everyone about herbs and Dharma rather than hand weaving and natural dyes. This is because her current job focuses on the self-reliance of rural women. Since farming is the main occupation of Prae Pun members, agricultural issues are important to help them meet the objective of self-reliance.

Kannika explained that, currently, Prae Pun has three active advisory committee members. She is responsible for financial and investment analysis, one member is responsible for hand weaving and natural dyes, and another one is responsible for agriculture. Advisory committee members' responsibilities are designed on the basis of advisors' skills and experiences. In fact, Prae Pun has one more advisory committee member, who is a NWD founder, but currently taking a break, as she is a senior. Kannika told the researcher that some of them have a break when they are tired. Another advisor put Prae Pun activities aside for awhile when she wanted to study Dharma. Kannika has been an advisor of Prae Pun for 10 years since NWD withdrew. She said that 
we came back to help them because we worried that they couldn't earn enough income to pay for the shop. They could do it within only two years. Then, they have been confident. I realise that they can do business... NWD cannot do weaving. Our role is to protect Prae Pun from going bankrupt. I am a committee member. I help Prae Pun in management.

The advisory committee members work for Prae Pun as volunteers. They do not get a salary but receive a committee meeting allowance which is the same amount as the committee members. Two of them are NGO workers in the field of gender development. One of them has become a farmer for self-reliance rather than for commercial reasons and the last one is a senior. They dedicate themselves to Prae Pun and use their free time to work for Prae Pun. They have to stay overnight at the showroom and office, using a traditional Isan mattress which is thin and small when they come to committee meetings, instead of staying at a hotel. They work for Prae Pun because they have had a relationship with the enterprise for a long time. Kannika told the researcher that

We do not feel that they are employees. We work together as relatives of each other. I can ask them to check the account without the feeling of inspection.... I work on this task without pay, even though Prae Pun has a budget. We have relationships with each other above the level of friends. We feel like sisters of each other.

Even though Prae Pun members operate its groups and businesses by themselves, it still needs support from advisors in many ways, including business ideas, information and encouragement. In fact, the advisory committee members fulfil the roles of information providing, training and analysing current situations. Kannika said:

I've a role to support them by analysing its financial issues ... We have open talk in committee meetings. I have to give them information, then, they have to make decisions by themselves.... Nowadays, our roles are less important. They used to listen to us, but 
now, they talk and discuss by themselves. They can stand without us. They have been developed.

As in the stories above, advisory committee members are crucial sources of external knowledge for Prae Pun, especially knowledge of business and group management. Indeed, the advisory committee members always share about hand weaving and natural dyes in the form of documents. As the advisory committee members are informal in their relationship with members, there is a lot of knowledge and experience sharing between the advisory committee members and Prae Pun, especially with committee members and office staff members through committee meetings.

\subsection{Chapter Summary}

It can be seen from the above stories, that Prae Pun is a rural community enterprise which utilises Isan culture to generate and operate its businesses. Prae Pun was established with the basic knowledge and skills in hand weaving and natural dyes of its members and the local wisdom of Isan societies_-villages and other hand weaving groups in Isan. Hand weaving and natural dyes, especially makleo, klang and kram, are traditional knowledge that Isan people have known how to use for many generations. These kinds of knowledge are transferred from generation to generation through traditional methods: teaching and telling of villagers, and weaving for domestic consumption and ceremonies. Prae Pun members have obtained that knowledge from their mothers and developed it to create unique products, and transfer it to the next generation mainly through traditional methods-teaching by mothers and senior members—and working.

Prae Pun has also obtained knowledge from many sources through study tours, training programmes and document study, and created new knowledge and skills which matches with its environment, resources and situations through experimentation, practice and internship. The working environment and styles of Prae Pun encourage members to obtain, share and transfer knowledge among members, committee members and customers. Prae Pun has many characteristics that influence and encourage knowledge processes including community-based, home-based, job sharing, open discussion, informal, collaborative and socialising. Prae Pun has the 
characteristics of a combination of Isan and NGO culture. Even thought the CE comprises seven village groups, members have the feeling of being the same community-Prae Pun. They work as a family rather than superiors and subordinates, and feel as if they are sisters. Thus, they help each other and are not possessive of their knowledge and skills. Those characteristics and its organisational culture enable Prae Pun activities to be considered as knowledge management, which will be discussed in the next chapters. 


\section{Chapter 6}

\section{Characteristics of Prae Pun}

\subsection{Introduction to Isan region and culture}

Prae Pun is a rural community enterprise involving a hand woven cloth business, located in Khon Kaen province in Northeast Thailand. The Northeast region of Thailand is known as "Isan" region and it has a unique culture - lifestyle, dialect, food, cloth, music and dance. Isan people live as a community or village which generally is called 'ban' or 'moo ban'. Households are located in groups and can be walked through from one to the other. This is because they have bamboo fences and open gates at the front side, and no fencing between houses. Villages in the same subdistrict are located not far from each other. There are paddy fields or other crop fields between villages, and connection roads. The main roads are normally cement or tar, meanwhile side roads are rocky. Traditionally, houses have two storeys with the ground floor a free space for general work, relaxing and social activities. Nowadays, many houses have only a single storey and are made of cement instead of wood. However, they still have free space in front of or beside the house for the same purposes. Figures 6.1 and 6.2 show the household layout and current home style of Isan.

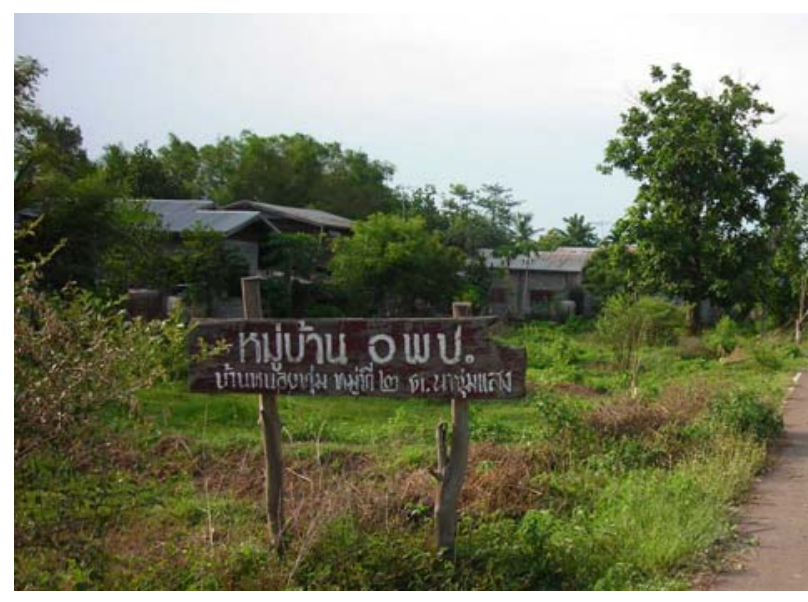

Houses are located in groups without fences separating each house.

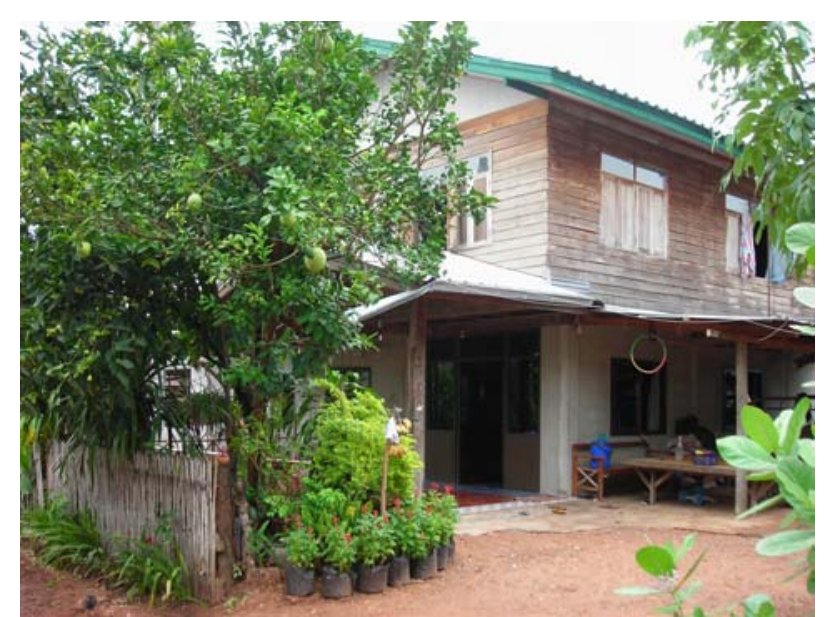

Current Isan home style with open gate and free space at ground level.

Figure 6.1 Household layout and current Isan home style. 


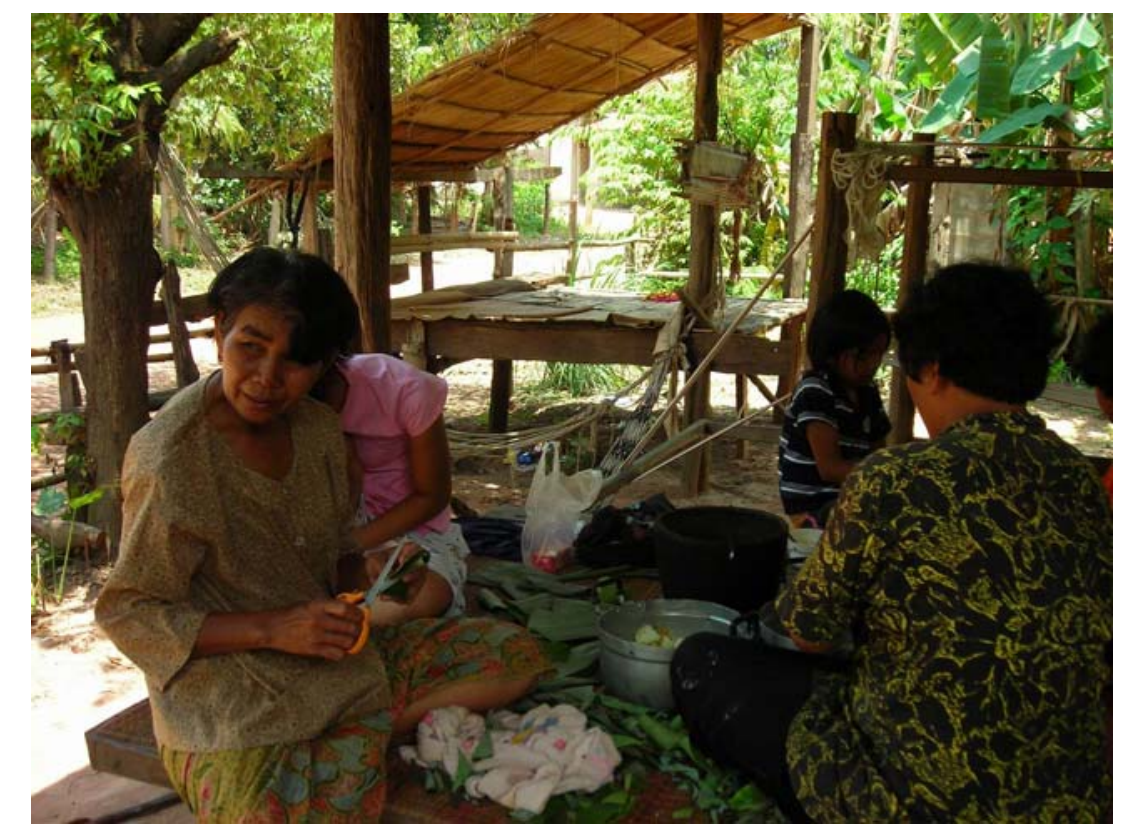

Figure 6.2 The ground floor of an Isan household has a traditional bench for relaxing, eating and socialising, and a hand weaving loom.

Villagers in the same village feel free to visit each other's homes to talk, and join in with ceremonial and social activities. This is because they have close relations and a belief in sharing the same spirit (Nartsupa and Lertwicha, 1994). Isan people believe that each village has the same ancestor spirit or 'Phee $\mathrm{Pu} T a$ ' located in the community to take care of community members (Kotkanta, 2006), and a paddy spirit or 'Phee Ta Hake' located in the fields to take care of cultivation (Baan Maha.com, 2007). Isan societies are oriented towards the group as their culture is concerned with inter-reliance (Katetade, 1997). From my own observations, villagers from the same village worship field spirits together at the start of the cultivation season. Villagers call each other with titles that show their relation, such as 'mae' or mother for married women, 'oey' or sister for elder women and 'eye' or brother for elder men. They feel free to ask for ingredients when they cook and invite their neighbours to have meals with them if they visit while they are eating. This observation confirms that Isan villagers in the same villages have close connections and inter-reliance.

The Northeast region of Thailand is a high plateau with dry areas. People mainly farm annual crops in the rainy season, such as rice, cassava and maize, and in the dry season they obtain supplementary work in other provinces. Generally, Isan women 
weave cloth for their family, and for ceremonial and income earning purposes in the dry season. One interviewee, Walai said that, nowadays, there are several groups hand weaving cloth for sale. In the current economic situation, women also migrate to work outside their villages in the dry season or even for the whole year. Many Prae Pun members stated that their daughters work in Bangkok and other provinces but may come back to settle at home villages when they have children. They will learn hand weaving at that time. This is because working in other provinces can generate regular and large income. In contrast, income from weaving is small and it takes longer to earn a large amount. This is consistent with Kamolwan's statement. She explained that some villagers have decided to work outside the village because they can earn faster. However, they prefer to live in village environment when they have a family. As mentioned previously, the village culture is inter-reliant. Relatives can help them take care of children when parents have to work on farms or go away on business. This is consistent with some members' situations, such as Raweewan and Kunya, who came back to settle down at their home villages when they married and had children.

Prae Pun can help the women of Isan solve problems and fulfil their self-actualisation needs and improve self-esteem. Prae Pun aims to develop hand weaving in order to make it a viable occupation for Isan women and a tool for local women's development. Hand woven products without high quality and styles to suit the markets would fetch low prices. For this reason, Prae Pun can set higher prices for its cloth in order to get fair prices for its weavers. Prae Pun runs businesses which focus on Isan culture-hand weaving and lifestyle. To more fully understand Prae Pun, we must consider the community's background, objectives, organisational structure and management, products and production, and marketing management.

\subsection{Background and Establishment}

Prae Pun was established as a community enterprise (CE) in 1992, with the support of the Handicraft Centre for Northeast Women's Development (NWD) which is an nongovernmental organisation (NGO) in the Northeast of Thailand working in the field of gender equality. In fact, Prae Pun was started around ten years before that by Mrs. Kanda Sakolkriet, who worked for NWD. She spent her free time working with two or three senior members of Prae Pun as a village member at Laokwienhuk village in the Muang district of Khon Kaen province, while she was working as an administrator 
for Save the Children in Khon Kaen. Mrs. Sakolkriet resigned from the Save the Children Foundation and founded the NWD in 1987. The NWD joined with three NGOs - the Save the Children Foundation, the Exchange of Resources for Community Development Project, and the Strengthening, Training, and Rural Development Project (STAR)— to work on Northeast women's development in eight villages in four districts of Khon Kaen province. These were Laokwienhuk in Muang district, Fangnoi and Nonthong in Nong Ruea district, Nongkham, Huafai, Nongthum and Nongyanlan in Phu Wiang district, and Suksomboon in Chum Phae district (Hutanuwatr and Hutanuwatr, 2002). Figure 6.3 shows locations of the eight original villages.

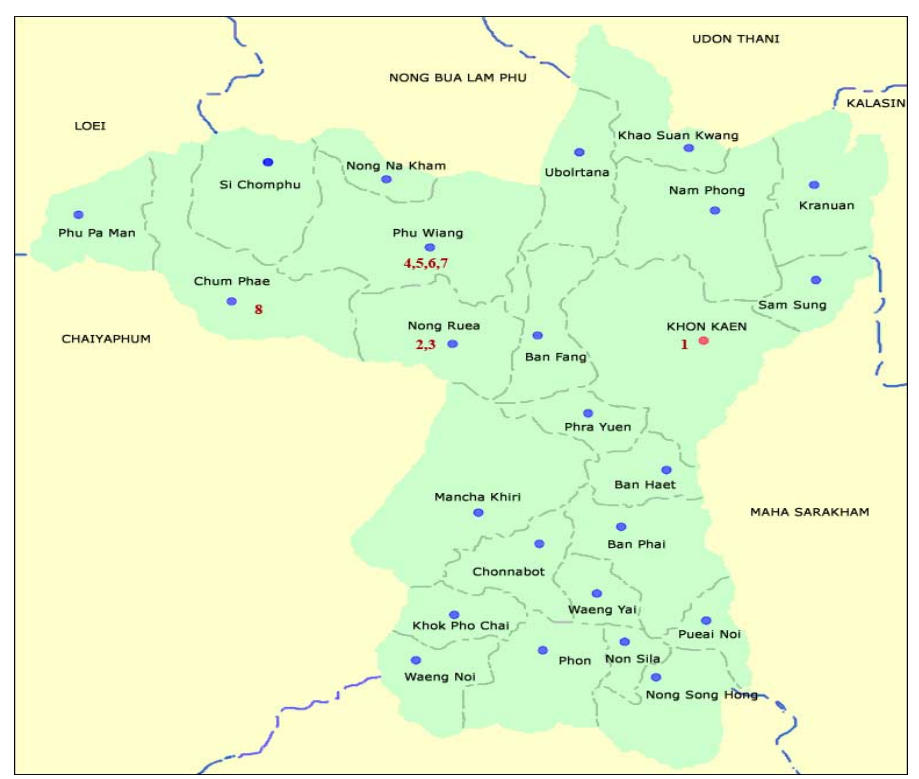

1 is Loakwienhuk, 2 is Fangnoi, 3 is Nonthong, 4 is Nongkham, 5 is Huafai, 6 is Nongthum, 7 is Nongyanlan and 8 is Suksommboon

Figure 6.3 Khon Kaen map with indicators showing locations of Prae Pun village groups

The narratives of this study indicate that NWD and its allies have conducted many activities for weaving and natural dyeing development, and for women's development, such as group management, literacy, health care and environmental conservation. Furthermore, Kannika—an advisory committee member-stated that NWD also conducted dharma study programmes and current political situation analysis for Prae Pun members in order to develop their minds and critical thinking. Dharma study has particular relevance for such gender development programmes 
because all the members are Buddhist. Honesty and ethical principles are criteria for selecting committee members, as pointed out by Walia. This context indicates that Prae Pun committee members have been recognised as displaying honesty and good ethics, and Prae Pun's operation and organisational culture are influenced by Buddhist philosophy and beliefs. The operational and management systems and organisational culture will be discussed in sections 6.4 and 6.5 .

In 1992, the women's groups from the seven initial villages with the exception of Nongkham village that did not want to join the community enterprise, decided to organise their own activities together as a cooperative under the name of "Prae Pun", a Thai word meaning especially beautiful cloth. Prae Pun started with 181 members with capital of 195,996 baht or around NZ\$ 8,100. The NWD was a major shareholder with 1,000 shares (Hutanuwatr and Hutanuwatr, 2002). After establishment, the NWD fully supported Prae Pun for two years in order to train members in business and group management, such as accounting, finance, stocking and selling, and customer coordination. Eventually, Prae Pun's businesses could be managed by its members, and it had an office and showroom in downtown Khon Kaen city with the help of a Japanese customer-Mr. K. Nawatabe, known as Mr. K from 'K. Craft for Life'. Kannika stated that Mr. K paid for his cloth orders in advance in order to help Prae Pun buy the office because he had made a high profit from Prae Pun's cloth. This demonstrates that Prae Pun does business with trust and good sense of customer relations., In this way, Prae Pun uses social capital as a resource for its business, as outlined by the concept discussed by Coleman (1988), and Nahapiet and Ghoshal (1998). The resources used in Prae Pun will be discussed in section 6.6.

Currently, in 2006, Prae Pun has around 200 members from seven villages as mentioned above. Its business functions have been undertaken by the members with advice from the advisory committee, and support from fair trade organisations (FTOs) and customers who are impressed by the hand woven cloth and Isan culture, and interested in rural women's development. Prae Pun organisational structure, and operation and management systems are going to discuss in section 6.4. 


\subsection{Objectives of Prae Pun}

Prae Pun was founded with four main objectives (Hutanuwatr and Hutanuwatr, 2002):

1) To encourage women's groups to be people-based organisations in order to increase their effectiveness in group, production and marketing management.

2) To influence self and community development in order to develop selfreliance.

3) To preserve and pass on Isan hand weaving.

4) To conserve natural resources and the environment through using natural dyes and replanting colour sourcing trees.

Regarding the first two objectives, Prae Pun conducts various activities including study tours, training activities, experimentation, and self-practice, to increase the quality of their products, effectiveness of group management and business growth. These activities can be viewed as knowledge processes, especially knowledge creation and capture, and will be discussed in chapter 7. Prae Pun continued these efforts until it could produce good quality cloth, and had leaders who are skilful at hand weaving and group management, and sufficient money to develop welfare programmes for members. Currently, Prae Pun focuses on in-house educational activities, such as on the job training, internship programmes and experimentation. Some new members, such as Pitsamai and Kunya stated that they only attend in-house training at Prae Pun member villages.

Currently, members have an average income of 2-3,000 baht or NZ\$80-120 /month/member from weaving. This can be seen from many members' statements, such as Prarichat, who said that, at the time of this research, Prae Pun had monthly quotas for members to earn equal income of about 3,000 baht per month.

For the third objective, Prae Pun focuses on the old Isan patterns, weaving techniques and natural dyes passed down from their ancestors and on to the next generation by teaching of mothers. The narratives of this study show that most members teach their daughters in order to help them and then their daughters would become members, and members who do not have daughters, such as Prarichat and Onanong, stated that they 
would teach their daughters if they had them. Members mainly weave traditional designs and patterns, but have adapted them to suit modern use. This can be seen from the use of kit and mudmee patterns (see appendix E) on many of their products, such as shawls, tablecloths, bags, etc. A good example is the members at Haufai village who weave big blankets, bedcovers and rugs with float-weave patterns used to make traditional blankets (Conway, 1992), but modified to suit with modern use.

Regarding the last objective, Prae Pun's cloth uses only natural dyes from bark, leaves, fruits, an insect substance (krang or stick lac), and other natural substances, such as rusty water and mud. Since Isan is a dry area, Prae Pun encounters a shortage of bark. Many members, such as Walai and Raweewan, explained that using a lot of bark from a particular tree would damage the tree. Therefore, members use a traditional method to support trees after cutting them, and also use mango and Indian almond leaves, ebony and annatto fruits, and stick lac instead of only bark for dyeing. In addition, a committee member from Nongthum village stated that the members in her village and Nongyanglan village have planted colour source trees together along the road between the two villages. Figure 6.4 illustrates the places of materials for natural dyes of Prae Pun.

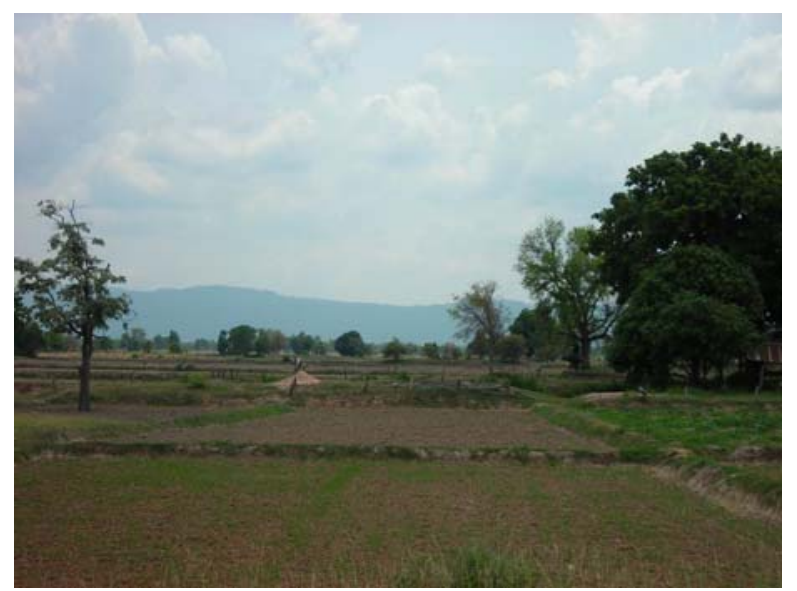

Some trees at paddy field. Compared with other regions, there are few trees in Isan areas.

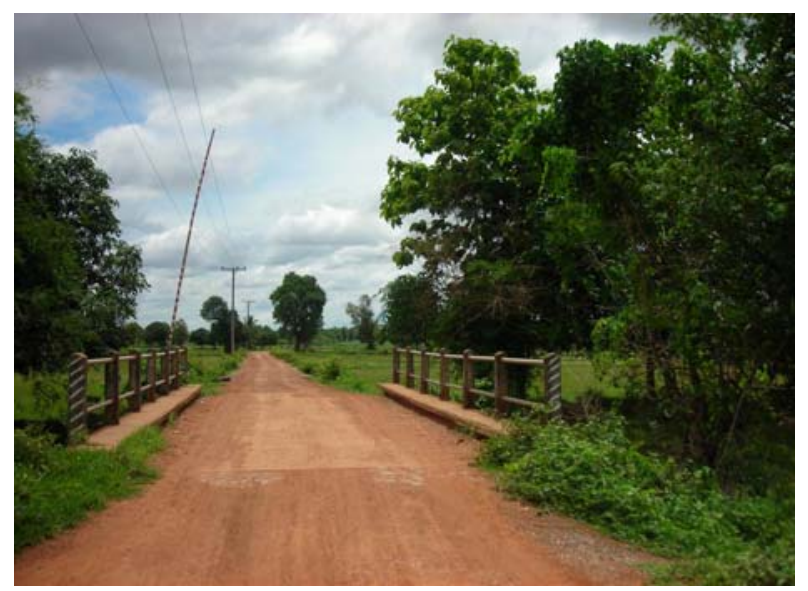

Trees beside the road between villages that Prae Pun members can use as materials for natural dyes.

Figure 6.4 Geography of the areas where Prae Pun villages are located

\subsection{Operational and management systems}

Prae Pun is not only a community-owned enterprise, but it is operated and managed by members themselves. Prae Pun manages the enterprise and village groups through 
committee systems, open discussion, self-employment and job sharing, and a homebased production system with unique product offerings and cloth quality standards.

\subsubsection{Committee systems}

Prae Pun has a board committee as its managerial unit, village groups as production units with village committees to operate their groups themselves, and an office as its administration and marketing unit. Figure 6.5 shows the organisational structure of Prae Pun.

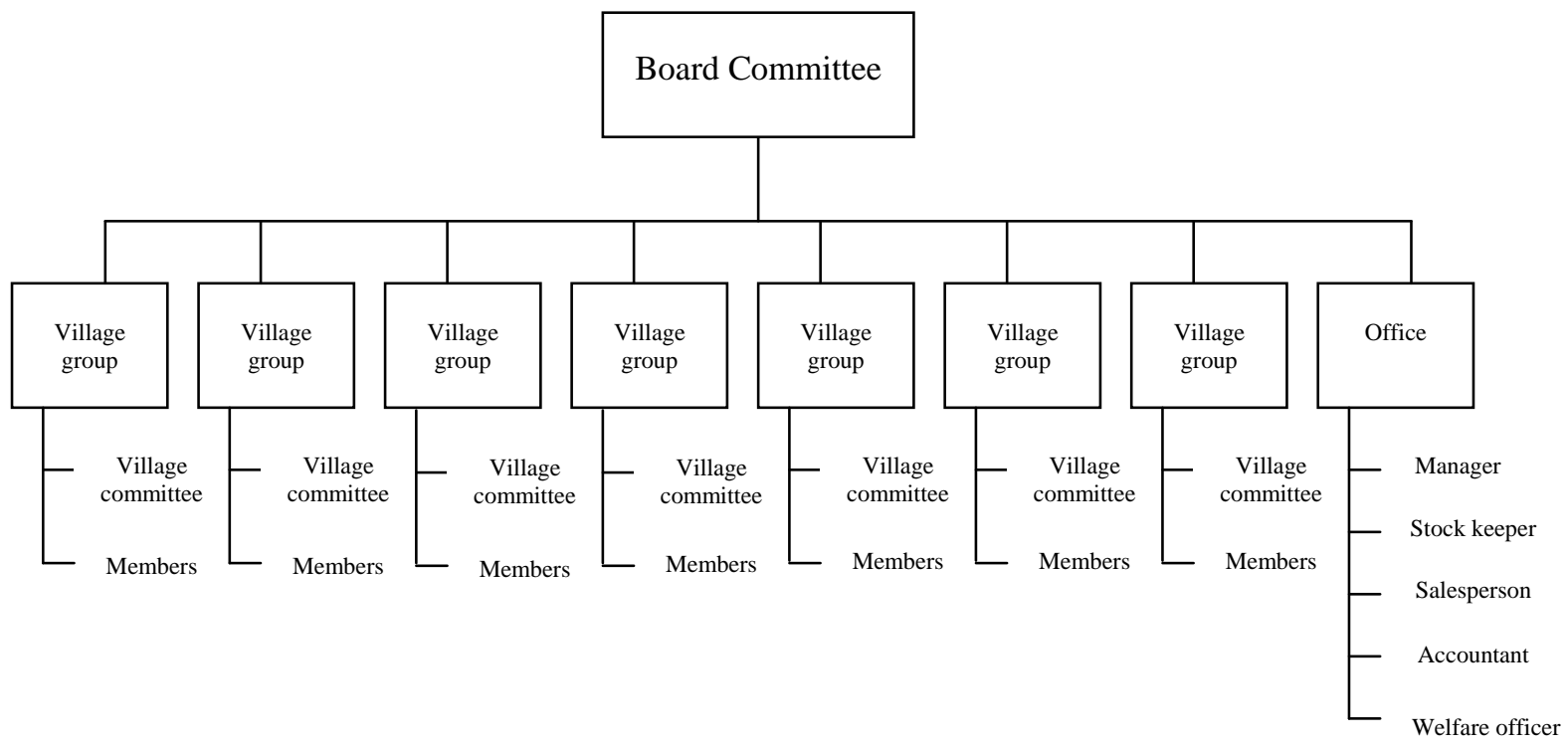

Figure 6.5 The organisational structure of Prae Pun

\subsubsection{Board committee}

Prae Pun's board committee has 17 members working together to manage all aspects of the CE. The board committee members can be divided into two groups: representatives of village groups and advisory committee members. Prae Pun has 14 representatives from the seven village groups as committee members. Each village group sends two members to be on the board committee of Prae Pun. One of them is chairperson of the village group and the other is normally a committee member of the village group. The data of this research show that village representatives are selected by the members based on their trustworthiness, honesty, middle age, literacy, ability in weaving, and ability to express ideas in order to identify problems and find solutions. They are skilled at problem solving and decision making. They also must be available to travel, work with documents, and advise new members about weaving. This is consistent with Napaporn's statement. She mentioned that she nominated her 
niece to replace her as a committee member because her niece is good at documentation and available for travelling. Furthermore, Napaporn's niece also revealed that she is good at hand weaving. She stated that many members invite her to teach them cotton bleaching and comment on their cloth design.

The narratives of this study suggest that committee members do not have only a role in managing Prae Pun, but they have also provide technical support for members. Napaporn stated that committee members have to attend meetings every two months in order to discussion Prae Pun’s situation and further activities. Meanwhile, Pitsamai and Kunya who are also committee members explained that they have to visit members in their villages to inform them about Prae Pun's news and policies, observe members' situations on behalf of the board committee and support them in hand weaving.

The second part of the board committee is an advisory committee which has three members who are representatives of NWD. They support Prae Pun in terms of management, production, and community and gender development. Kannika explained that advisory committee members are assigned to focus on different issues. The first member concentrates on weaving and dyeing techniques, the second member conducts financial, situation and investment analysis, and the third member focuses on sustainable development through agricultural issues. This is because Prae Pun members are involved in agriculture. However, Kannika stated that the main role of advisory committee members is as information providers in order to extend the worldviews of the committee members. Meanwhile, the main decision-making is done by the committee members themselves.

Prae Pun has regular board meetings every two months and an annual meeting among the seven village groups in order to report operational results, and obtain feedback and suggestions from members. The committee works together to manage Prae Pun without set terms. This is because they work with the trust of each other based on their relationships as friends. Kannika observed that committee members have worked for two or three years without changing, because they feel like sisters rather than employees. Prae Pun has its own management systems as confirmed by Kannika's statement. She expressed that they do not want a western system where members only 
have a term of only two or three years, because new committee members need to learn from senior committee members.

From my own observations, the committee members did not approve the resignation of the chairperson who had been in charge for over 10 years. This is because they trust her judgement and need her experienced guidance. Prae Pun not only relies on the experience of senior committee members, but also their trust and moral support. This shows that Prae Pun utilises social capital for its management system, in line with Hoffman, Hoelscher and Sherif (2005) because Prae Pun utilises trustworthiness to facilitate the community's activities. This is consistent with other micro enterprises

in Thailand, such as some community enterprises in Northeast Thailand which have also been found to trust in managerial teams for the success of their management systems (Huntrakul, 2000).

\subsubsection{Village groups}

Prae Pun comprises seven village groups located in Khon Kaen province. These village groups are considered production units working under Prae Pun's strategies and policies. While each village group has its own management system, each is quite similar with a village group committee to operate and manage the group. The village committees are made up of different positions. Generally, they have a group chairperson, secretary and treasurer. For instance, the Suksomboon village group has a position responsible for public relations to promote weaving techniques to members. Each group has regular meetings, especially after the board committee meetings, in order to catch up on what has happened there, and each has its own financial management system. Generally, they charge about two to three per cent of the price of the cloth sold by members as income to cover the group's expenses, including cloth delivery to the office, committee allowances and hospitality for guests. Raweewan explained that her group also charges members two per cent of their cloth delivery for shipping cost and group activities.

Each village group has a sub-committee, known as a cloth inspection committee. The cloth inspection committee has around three members, working together once a month to check members' cloth before sending it to the office. This committee provides quality control, which is a part of the production system of Prae Pun (see section 
6.4.5). In some village groups, every member takes a turn sending her products to the office in order to observe other members' cloth and Prae Pun activities and to build up the feeling of ownership. Furthermore, the village committee has an important role in supporting members. They have to help members meet cloth quality standards, and praise them in order to encourage them to continue working. Lada-a skilful committee member at Suksomboon-mentioned she often visits members in her group in order to help them solve weaving problems and design cloth. Meanwhile, Kunya - a young committee member at Nongyanglan—stated that she also has to visit members who live near her home in order to encourage them in weaving. On this basis, the village group committee members are as skilful as the board committee members, but the village group committee members are more concerned with technical support rather than the CE’s management.

\subsubsection{Members}

Members are the part of the village groups. They have the role of producers. The majority of Prae Pun members are women who are married with low levels of education. A few of the women's husbands also belong and weave together with the female members. The majority of female members are in their 40s, some are over 50 years old, and some in their 30s. A study by Tshering in 2003 found that few members were under 20 years old. The narratives of this study and my own observations indicate that there are still only a few members younger than 30, new members who have just married and returned to settle down in their home villages. Most of them begin their connection to Prae Pun by helping their mothers to weave, and then taking over their mothers' memberships. Kunya stated that she became a member when she came back to her home village and started weaving with her mother and sisters who are Prae Pun members.

Prae Pun has not recruited new members for some years. This is because Prae Pun sold fewer products during the period of economic crisis and also since then. However, it needs new members from the younger generation to preserve its weaving techniques and Isan culture. This is because senior members are having difficulty with some tasks. For instance, Raweewan and Onanong mentioned that nowadays, they cannot do kleab kao because their eyesight is not good enough. Meanwhile, Urai said the she wants her daughter to help, because making natural dyes involves heavy 
labour. New member recruitment is approved by the board committee after consideration of the number of current members in each village. Many members stated that new member selection is based on applicants' weaving skills and attitudes, and economic status. Prae Pun gives priority to poor women and the daughters of existing members. Prae Pun targets the poor because it has the objective of women's development, which includes providing income for women (see section 6.3). In terms of skills and attitudes, Prae Pun focuses on women who enjoy hand weaving and target members' daughters. This is because hand weaving is difficult and requires inspiration and enthusiasm. Kamolwan stated that Prae Pun needs members who enjoy hand weaving because the craft requires patience and artistry. Prae Pun focuses on members' daughters because they can learn the basic skills and absorb appropriate attitudes from their mothers. This is confirmed by Suwanee, who stated that normally new members are our daughters whom we teach to weave for us when we are busy. In terms of Prae Pun organisational culture, the members' daughters assimilate Prae Pun values from their mothers and again while they are working for Prae Pun. This is shown by Urai's statement that her daughter often works with the cloth inspection team as her substitute.

\subsubsection{Office}

The office is Prae Pun's administration and marketing unit, based in downtown Khon Kaen city. The office works under the board committee policies and has a manager as a supervisor. The office comprises five staff members: manager, accountant, stock keeper, sales person, and welfare staff member. Currently, it has only four staff members, so the manager and the stock keeper also have to work on accounting, and every staff member has to deal with ordering and selling. Penchun stated that every staff member is able to take orders, while one staff member is in charge of international orders, and the manager is in charge of large and new product orders. For off-site selling, every staff member takes turns at the handicraft fairs while the sales staff member is responsible for selling in Prae Pun's showroom. On this basis, the office work clearly entails job sharing (see section 6.4.4).

Prae Pun is similar to other micro enterprises in Thailand that also manage their enterprises with committee systems (Wasuntiwongse, 1999). Prae Pun's committee systems represent collaboration and decentralisation, according to the concept of 
organisational structure and centralisation outlined by Hall (1982), because the committee systems of Prae Pun allow members to participate in management and decision-making through group meetings and their representatives-committee members - have full authority to share members' situations and make decisions. Furthermore, the committee systems of Prae Pun are concerned with trust and willingness to work for Prae Pun. Village groups and the office share responsibility in particular areas. The village groups are responsible for production, and office for marketing. The findings of this research broadly support the statements of Gibson, Ivancevich and Donnelly (1994) that organisations that delegate authority downwards to other units, such as village groups in this case, are practising distribution decision making.

\subsubsection{Open discussion}

The working style of all levels - board committee, the office and village groups-is open discussion. Members and office staff members feel free to communicate with each other, and with committee members and office staff members, because they are all villagers and familiar with each other. The narratives of this study and my own observations show that members mainly share their opinions through village group meetings, committee meetings and informal socialisation while visiting each other. However, new members are often less familiar with expressing ideas to the groups. Some of them keep quiet because they are younger and have less experience than long-term members. This reflects the impact of Isan culture and the Thai value of kreng jai. Isan culture requires people to respect their superiors in knowledge and ethics (Katetade, 1997). Similarly, the Thai value of kreng jai honours those with higher rank and seniority (Holme and Tangtongtavy, 1997). Meanwhile, long-term members are more familiar with Prae Pun culture as they have been trained in idea expressing and critical thinking by NWD. Kannika-an advisory committee member-said that NWD trained members through a series of programmes, including gender issues and occupation issues. These sessions helped members to express their ideas and make decisions by themselves. This shows that the open discussion system of Prae Pun is influenced by NGO culture, because, as mentioned in sections 6.2 and 6.4.1, NGOs have been the originating organisations and providers of ongoing business support in the form of group and business management training activities. As evident from the literature review, NGOs' main focus is on fostering participation and 
decision-making (Bernard, 1984; Paton and Cornforth, 1992) and they are accountable to many stakeholders (Hudson, 1995). Furthermore, members also feel free to discuss with advisory committee members who are NGO workers because of the NGO characteristics of participation, decision-making, and informality (Bernard, 1984; Paton and Cornforth, 1992).

\subsubsection{Self-employment system}

Prae Pun operates its enterprise through a system of self-employment instead of hiring skilful staff for both the office and village groups. In the office, all staff members are Prae Pun members or their daughters. Penchun explained that Prae Pun invites members to be interns at the office in order to encourage members to be office staff. Prae Pun wants office its staff members to have basic knowledge of hand weaving and loyalty to Prae Pun. However, most interns do not want to work in the office because, as villagers, they are not familiar with the city life style. This is shown by Malee-an ex-manager - who mentioned that she had to practise how to talk with customers and answer the telephone when she worked at the office. Nevertheless, office staff members feel relaxed because working at the office is flexible. They work without contract, and can resign from the office and become weaving members in the village groups. Malee said she had to stop being an office staff member several times because she was busy with farm work. This shows that Prae Pun is flexible and operates with informal characteristics which are a characteristic of other micro enterprises. This fits with the findings of Halvorson-Quevedo (1992) and Wasuntiwongse (1999) that micro enterprises tend to conduct businesses with casual employment rather than formal contract.

The village groups, on the other hand, have no staff. Members are self-employees who earn income from their cloth instead of a salary or wage. This matches with Wasuntiwongse (1999) study, which found that members of community enterprises in Thailand are self-employed and work at their homes or community places. Each village group has a committee member, and members have a rotation scheme to help the committee members for tasks, such as monthly cloth delivery and welcoming guests. Furthermore, members have to help office staff members in cloth selling at exhibitions, because. Penchun explained that this requires at least one member to be present to demonstrate demonstration. 


\subsubsection{Job sharing}

As discussed in section 6.4.1.4, the office has only four staff members. Each staff member has her main duties. However, each also has to help the other staff members with their duties because some tasks require more than one staff member. Some tasks also require involvement from members from village groups to do, such as selling and demonstration at exhibitions. This job sharing is due to the fact that Prae Pun has limited numbers of staff, budget and available human resources. This is consistent with some members' statements, such as Kannika stated that Prae Pun needs staff members skilled at marketing and hand weaving but cannot pay high salaries for those positions. The findings of this research support the findings of Trade and Investment Division (2003) that workers of rural-based enterprises tend to lack skills at business management because they have a low level of education and few opportunities in ability development. Wasuntiwongse's (1999) study showed that micro enterprises in Thailand tend to have little capital and therefore often use a self-employment system.

\subsubsection{Production system}

The production of Prae Pun is a home-based system with unique product offerings. First, members work in their homes without set hours, This is the same as other micro enterprises in Thailand, where that production takes place at members' homes or communal areas, such as community halls and temples (Wasuntiwongse, 1999). However, the production of Prae Pun is controlled by a quota system and cloth quality standards. The narratives of this study show that the quota system has been adapted in order to maintain sufficient stock and to provide equal income distribution to members. Furthermore, the quota system controls the quality of the products as some members tend to focus on income rather than cloth quality and skill improvement. For example, Napaporn noted that some members are careless when they speed up their weaving. Therefore, the quota system can control members to work at the optimal speed to ensure they pay attention to cloth quality. Prae Pun has also standards to control the quality of cloth and set prices. Kamolwan explained that low quality cloth would receive lower prices, be accepted only on consignment, or rejected by the office. In the worst cases, some members might be asked to stop weaving.

Prae Pun's production is based on the CE's unique product offering and skills Members are free to design their cloth but are given directions from the office about 
customer preferences. Generally, members follow established designs according to samples from neighbours, members, and the office. Onanong stated that she normally asks for sample designs from the office because the office staff knows customer preferences better than members at villages. In terms of the plaid and stripe cloth, every member had to design it themselves, but the narratives of this study show that new members find designing quite difficult. They need other members' ideas and comments in order to design their cloth. Pitsamai and Lamai mentioed that other members ask them to help or make them comments in order to modify the designs. Skills are important for a production system with unique product offerings. Furthermore, each village group produces different types of cloth in order to provide varieties of products for Prae Pun and maintain its stock. Each village group has been assigned to weave different types of cloth based on the particular skills and environment of each village group. For instance, as Malee mentioned, members at Nonthong group weave only silk cloth. Meanwhile, members at Huafai village weave blankets, towels and rugs with float patterns because they are familiar with blanket weaving. The fact that members choose to work based on their skills and experience agrees with the findings of Nelson (1987), and Halvorson-Quevedo (1991), that micro enterprises mainly do businesses based on artisanal techniques and local skills. Because the production focuses on skills of members, Prae Pun pays close attention to skill development through a series of activities, which will be discussed in chapter 7 .

\subsubsection{Marketing management}

The narratives of this study and my own observations suggest that the marketing of Prae Pun's products is quite specific and targets a niche market providing an alternative to mass-produced items. This is because a Prae Pun's marketing strategy is focused on traditional and environmental-friendly products, and trading by the weavers themselves. Prae Pun's customers are high-end consumers who buy Prae Pun's products with an understanding and appreciation of the products' purpose to preserve the Isan culture and environment. It is believed that this in turn will support the local Isan women. The main markets of Prae Pun are FTOs and domestic and international green markets. In terms of the domestic market, Prae Pun has a showroom at its office in downtown Khon Kaen city, and primarily stocks handicraft fairs, and sells products through ThaiCraft, which is a FTO in Thailand and Lemon Farm which is a green shop in Thailand. Wijittra commented, however, that Prae Pun 
had stopped selling at general handicraft fairs because it experienced less success selling to general customers. As a result of the 1997 economic crisis in Thailand, there are a lot of cheaper hand woven products on the market with similar patterns and designs. Therefore Prae Pun has had to search for more markets through its current connections. Besides retailing with ThaiCraft, Prae Pun has obtained new wholesale customers at the fairs and through ThaiCraft. From my observations, the current customers also help Prae Pun to expand its market by conducting small exhibitions and selling on their own sites. For international markets, office staff members and many members state that Prae Pun has a wholesale contract, selling cloth to Japanese customers who focus on hand weaving products.

In terms of prices, compared with the common hand woven cloth in the markets, Prae Pun's products are expensive. Kannika explained that this is because Prae Pun has determined the prices for the benefit of the weavers, to cover the costs of production which is time-consuming and requires specific indigenous skills, creative ideas, and scarce natural materials, Therefore, Prae Pun customers are willing to pay more to buy artisanal products as part of fair trading for both environment and producers.

The operation and management systems of Prae Pun focus on decentralisation and collaboration, as the CE comprises seven village groups working together with committee systems, open discussion and job sharing. The production units are the village groups with members as self-employees who gain income from their cloth. The production takes place at members' homes, involving unique product offering, the quota system and cloth quality standards. The products are sold in niche markets through FTOs and green market.

\subsection{Organisational culture}

Because Prae Pun is a community-based enterprise supported by NGOs, its organisational cultures are influenced by village culture, Thai culture and NGO culture. Furthermore, Buddhist beliefs also influence Prae Pun because all Prae Pun's members are Buddhist. The organisational cultures of Prae Pun comprise community spirit, sense of ownership, and informal participation. 


\subsubsection{Community sprit}

Both the office and village groups display community spirit. Even though Prae Pun comprises members from seven villages, office staff members come from several village groups and advisory committee members are NGO workers, all of them feel a sense of belonging to the one community-Prae Pun. This culture is further influenced and reinforced by Isan culture, Thai culture and Buddhist beliefs.

\subsubsection{Influence of Isan culture}

Prae Pun has drawn upon its Isan culture to create a community rather than a business. The Seven village groups and office are viewed as one community sharing the same community spirit as Isan culture. Villagers are united and help each other, because they have close relations and the same culture. Isan people believe each village has the same ancestor spirit or 'Phee $\mathrm{Pu} T a$ ' who takes care of community members (Kotkanta, 2006), and a paddy spirit or 'Phee Ta Hake' to take care of cultivation (Baan Maha.com, 2007). As mentioned in chapter 5, each village holds a ceremony to worship Phee $\mathrm{Pu}$ Ta and Phee Ta Hake together at the beginning of cultivation season.

Members from the seven villages groups have the feeling of belonging to the same community because they have the same status as members of Prae Pun, do the same tasks for Prae Pun, have to follow Prae Pun's regulations and gain benefits from being members. Many state that they have to accept Prae Pun rules when they become members. A good example is Onanong, who said that, when she moved to Nonthong village, she had to learn how to weave silk and only silk, because that was the task for that village group. Social identity theory explains that people tend to classify themselves as belonging to a group based on their perception relative to individuals of the group and the benefits obtained from the groups, and have to conform to the group norms accordingly (Ashforth and Mael, 1989).

In terms of benefits, members can obtain both tangible benefits-income and welfare-and intangible benefits—self-satisfaction. Members are proud of being part of Prae Pun because they are recognised as good weavers and can fulfil their selfactualisation and self-esteem needs. They can earn extra income from weaving to support their families, undertake activities for pleasure (weaving) and demonstrate 
their abilities in businesses. This can be seen from the statement of Prarichat, a committee member, who pointed out that Prae Pun was able to maintain its business during the economic crisis whereas other groups collapsed.

As with the village group members, office staff members also have the feeling of belonging, as shown by Kannika's comment that office staff members do not feel they are employees. They can share their opinions to manage Prae Pun the same as members. From my own observations, the office staff members have the feeling of being the members of Prae Pun because they also contribute their hand weaving knowledge. For instance, the manager used to be a weaving member, and now uses her experience for dealing with customers and members in order to maintain orders and cloth qualities. Kamolwan explained that the manager can decide the price of specific orders for members without having to check the working time of members because she knows the process of weaving well. The office staff members are also proud of being able to perform business activities. Penchun told me her experience of being a staff member and learning office duties with pride because Prae Pun has given her opportunities to develop business skills.

Significantly for this study, advisory committee members also feel a part of Prae Pun because they have also deal with hand weaving and enterprise management (see section 6.4.1). Kannika, an advisory committee member, stated that she works for Prae Pun because she has a social relationship and a feeling of being related to Prae Pun's members. Even though NWD withdrew from Prae Pun in around 1994, NWD’s staff members have come back to work with Prae Pun as advisory committee members. This is also consistent with the social identity theory and the finding of Hutanuwatr (1998) that community members share the same activities, obtain mutual benefits and encounter the same obstacles.

Therefore, belonging to the same Isan culture and community influences members to help each other in that villagers have close relationships and inter-reliance (Nartsupa and Lertwicha, 1994). Penchun —an office staff member-mentioned that they work in the office as a family. They do not have a boss and subordinates. Kunya remarked that she can ask skilful members in her village to fix her weaving problems at her place. She can also turn to members in other village groups as well, if the members in 
her village cannot help with those problems. From my own observations, office staff members also seek some help from each other. They also accepted me as a temporary member of their community, by inviting me to join many activities in both office work and socialisation, and also asking me for some help.

\subsubsection{Influence of Thai culture}

The sense of belonging is also reinforced by the Thai culture of interdependence and the value of kreng jai or consideration. Most Prae Pun members are not possessive of their cloth designs and are willing to help others solve weaving problems. Members do not hoard knowledge only for themselves, but realise their knowledge can benefit other members. Thai culture entails vertical collectivism, according to Atmiyanandana and Lawler (2003), and people in such societies tend to have a sense of serving and sacrificing for the benefit of the in-group. The degree of kreng jai is less formal amongst people who have a close relationship. As a result, members that identify themselves belonging to the same community are comfortable within their in-group in expressing opinions, requesting for help and working together.

\subsubsection{Influence of Buddhist beliefs}

Prae Pun's sense of community can also be explained by the Buddhist beliefs of sharing and non-attachment. As revealed by Boonmathaya's (2003) study, villagers in northeast rural Thailand have strong Buddhist values and willingly share both materials and knowledge. Because all members of Prae Pun are Buddhist, they are influenced by the beliefs of compassion, sharing and non-attachment to materials.

\subsubsection{The sense of ownership}

Prae Pun's members not only have the feeling of belonging to the same community, they have a sense of ownership which influences members' strong commitment to working hard, searching for knowledge, and developing their skills and abilities. This study suggests that Prae Pun has created a sense of ownership through both behaviour and cognitive processes. First, members are involved as the owners of Prae Pun. This can be viewed as a sense making through behaviour process according to Trice and Beyer (1993), because members are involved in activities. Kannika stated that all the original members signed the loan agreement to buy a building for the office and showroom together, in order to make them the owners of the building instead of only 
the committee members. Since then committee and senior members have tried to create the sense of ownership for new members by allowing them to visit the office and showroom in Khon Kaen. In a wider view, this can be described as sense making through the cognitive process according to Trice and Beyer (1993), because members are able to explore and perceive situations by themselves. Napaporn mentioned that committee members want new members to consider the office as their property, andf the monthly cloth deliveries give them opportunities to visit the office in order to let them experience the feeling of being owners. Members are aware of their status as the owners of Prae Pun. For example, Suwanneee remarked that she often visits the office when she visits Khon Kaen. She feels that it is also her home because all of the 200 plus members are owners.

As members share the ownership of Prae Pun, they have high commitment and are willing to work hard and learn in order to build their capabilities for working. The narratives of this study reveal that members pay close attention to acquiring knowledge of hand weaving and natural dyes, and making quality cloth. Prarichat mentioned that she found out how to make good selvage by trying out a variety of work processes in order to make quality cloth. Malai also indicated that members do not deliver low standard cloth to the office because they know it will not be sold and will be a burden for the office. Meanwhile, committee members and office staff members also practise in terms of business operation and management, such as stock maintenance and finance and investment, which are far from their experience. They want to operate the enterprise by themselves rather than hiring professional staff to work. Penchun stated that she prefers members or daughters of members to work as office staff members rather than external skilful employees, because they have this sense of ownership and understand Prae Pun.

It is evident from the literature review that sense of ownership leads to greater involvement, responsibility and commitment by organisational members (Gopalakrishnan and Santoro, 2004). From the perspective of sociology, the sense of ownership of Prae Pun can be viewed as high solidarity, according to organisational culture concept suggested by Goffee and Jones (1999), because members focus on high commitment, shared objectives and goal achievement. In a broader view, the sense of ownership and high commitment of Prae Pun contributes to a result-oriented 
culture, according to organisational dimension suggested by Hofstede (1991), because members focus on goal achievement.

\subsubsection{Informal orientation}

The narratives in this study suggest that both village groups and the office have an informal style that involves sharing among members, staff members, customers and supporters. This culture is influenced by both Thai culture-mai pen rai-and NGO culture. The value of mai pen rai or 'does not matter' (Komin, 1990; Dubey-Villinger, 2001; Atmiyanandana and Lawler, 2003) encourages members to be flexible and informal. From my own observations, rural village members are very flexiblyoriented and display low uncertainty avoidance, with few set rules governing their behaviour. This is opposite to Hofstede (1991)'s study which found that Thai society displays high uncertainty avoidance at 64 per cent, possibly due to the close community relationship between Prae Pun's members. Another influence is that of NGOs which have an informal working environment for both work places and NGO workers (Hudson, 1995). This is consistent with my own observations that the advisory committee members who are also NGO workers are comfortable to work by sitting on the floor and stay overnight at the office without bed and mattress.

In the village groups, members work at home without time control and feel free to switch from weaving to family work, farm work or socialisation. Members can do many activities including developing their skills and interests and socialising at the same time, thereby expanding their knowledge and abilities. Their working places are located on the ground floor or in small huts that make it possible for other members and villagers to visit and socialise. Furthermore, the narratives reveal that currently most activities take place in members' villages in an informal atmosphere that allows participants to relax. Many members talked about the natural experimentation that took place at Nongyanlan village last year. From my own observations, committee member meetings are conducted in an informal style at the office with members sitting on the floor, offering many opportunities for informal conversation.

The office has also an informal and rural style. Office staff members bring their village culture to the office. From my own observations, the office has the feeling of a home rather than a corporation. From the physical aspect, Prae Pun office has been 
designed in the traditional Isan home style - gate decoration and showroom style with bare wooden floors for seating. As described in chapter 5, the informal style of Prae Pun is the Isan life style. Members mainly sit on the floor or simple traditional bench. Villagers feel free to visit to socialise and ask for help, and the office staff members are informal and flexible. They like to work in the open space (showroom) rather than in the office. Office staff members feel free to ask their manager for advice and for other members to help them with some tasks. As discussed in the previous section, office staff members do not feel like employees. As Kannika observed, the office has flexible office hours and holidays with a rotation system and goodwill of the manager and staff members. This means the office and showroom can remain open seven days a week with no resentment from the staff members.

In a wider view, the informal orientation of Prae Pun can be viewed as loose internal control, according to organisational dimensions suggested by Hofstede (1991). This is because Prae Pun is more concerned with village culture rather than strictly formal rule as a business sector. Seen from the perspective of sociology, the informal orientation of Prae Pun can be viewed as high-sociability culture, according to Goffee and Jones (1999) because Prae Pun has an environment of friendliness, sincerity and freedom in its sharing opinions.

\subsubsection{Participation orientation}

Prae Pun organisational culture is also participation orientated. As discussed in section 6.4.1, the committee systems allow members to participate in management of Prae Pun. Members have representatives on the managerial team and all members have opportunities to share their opinions through many activities, such as group meetings, annual meetings and sharing forums. The analyses of the narratives in this research suggest that long-term committee members who are familiar with Prae Pun culture express many opinions at committee meetings. However, new committee members are reluctant to speak out, until they are more familiar with Prae Pun culture. Isan culture also means that people have to respect superiors in knowledge and ethics (Katetade, 1997). This is also because of the Thai value of kreng jai or consideration toward people who have higher rank and seniority (Holme and Tangtongtavy, 1997). 
Nevertheless, new committee members undergo training on Prae Pun culture. This is because, as Napaporn explained, members need to know how to discuss in order to gain group consensus. Kannika —an advisory committee member—said that NWD training programmes provide opportunities for the committee members to express their ideas and make decisions themselves. It can be seen that the long-term committee members who attended several training with NGOs are particularly familiar with sharing and expressing opinions. This suggests that Prae Pun is influenced by NGO culture, with its strong focus on participation in activities and decision-making (Bernard, 1984; Paton and Cornforth, 1992). In a wider view, the participation orientation can be viewed as open communication, according to organisational dimension suggested by Hofstede (1991), and as a high-sociability (Goffee and Jones, 1999) because members have social interaction and freedom in sharing opinions.

Altogether, the organisational cultures of Prae Pun consist of a community spirit, a sense of ownership, an informal orientation and participation orientation influenced by Isan, Thai and NGO cultures. These values influence Prae Pun members' informality, friendliness, socialisation, participation and open discussion, and high commitment. This also reveals its organic culture, according to organisational culture of small enterprises discussed by Wong and Aspinwall (2004), because Prae Pun is concerned with a flexible systems, task orientation, minimal direction and open-space workplaces. In terms of operational aspects, the organisational culture of Prae Pun can be viewed as result-orientate, with loose internal control and open communication, according to Hofstede's organisational culture dimensions (1991). From a sociological perspective, in accordance with Goffee and Jones (2999), Prae Pun's organisational culture is one of high sociability and high solidarity.

\subsection{Resources}

Prae Pun has limited finance and technologies, which are constraints for its business and knowledge management. As discussed in section 6.4, Prae Pun cannot afford skilful staff members or office manager. Prae Pun relies on local resources including funding from members and local knowledge of hand weaving, representing human social capital. Nevertheless, Prae Pun also utilises ICTs for some particular activities 


\subsubsection{Prae Pun's knowledge}

Prae Pun's knowledge focuses on hand weaving, natural dyes and community enterprise management. Most Prae Pun's knowledge is derived mainly from indigenous craft knowledge adapted to Prae Pun's situation over 10 years.

\subsubsection{Knowledge of hand weaving}

Members possess knowledge of hand weaving in several forms including skills, experience, observations and creativity which can be viewed as both declarative and procedural knowledge. First, all members must have at least basic skills at hand weaving as a requirement of joining Prae Pun. These basic skills are procedural knowledge according to characteristics of knowledge interpreted by several authors, such as Sanchez (1997) because they represent the ability to perform activities. Members mainly acquire this knowledge with traditional methods-learning from mothers or woman villagers when they are teenagers in order to make cloth for family consumption and ceremonial purposes. For example, Mali states that she learnt how to weave when she was 11 years old. Her mother first asked her to weave cotton plain cloth, then, she learnt how to make kit for pillows. After joining Prae Pun, members acquire further knowledge and also create new knowledge of all forms.

Second, members, especially long-term members, also have advanced skills at hand weaving. As with basic skills, advanced skills also constitute procedural knowledge because they are derived from good practice over a period of time (Sanchez, 1997; Nahapiet and Ghoshal, 1998). The narratives of this research reveal that members can make several types of cloth, ranging from the simple plaid and strip cloth, to advanced patterns, such as kit, mudmee, float patterns, 3-kao weaving techniques. Kunya mentioned that her village group weave place mats with the 3-kao technique which is complicated because it requires controlling the loom with three foot pedals. Meanwhile, Raweewan explained that members at her village group are skilful at making big blankets with float patterns, with each member skilful at particular designs.

Third, members obtain experience and observations from study tours, training activities and working processes. The information obtained from those activities can be viewed as declarative knowledge because it is concerned with facts and 
perceptions of members without practical engagement. This knowledge type requires practice in order to search for procedures and skills (Ryle, 1949; Varela et al, 1995; Brown and Duguid, 1998). Raweewan mentioned that a senior member in my group saw jug jig design from a study tour, but had to practice for a period of time in order to search for the appropriate heddle shaft preparation to make the design.

Four, the narratives of this study reveal that members, especially skilful members, display creativity for new designs and products. The creativity is derived from a combination of creative ideas, particular skills and task motivations (Amabile, 1983; Kilgour, 2006). For example, Kannika mentioned that Prae Pun has more kit designs than the common Isan kit designs because members have adapted other patterns as well. This process requires a certain skill level in terms of kit, other cloth patterns and local production strategies. As with experience and observations, the creativity can be viewed as declarative knowledge because it is involves the state of thinking where members have to search for procedures to make products. For example, Raweewan explained that she needs to search with a trial and error method in order to make her own particular designs.

\subsubsection{Knowledge of natural dyes}

As with knowledge of hand weaving, knowledge of natural dyes encompasses the same forms and is acquired with similar methods. In terms of basic skills, members acquire that knowledge from training activities and other hand weaving groups, through study tours, and practice and conduct experimentation. This is because villagers in Prae Pun villages stopped making natural dyes a long time ago. However, many still remember and share this knowledge with members. Kamolwan stated that senior villagers at her village told her the same natural dye making method as she has since learnt from NGOs. Most members stated that they also learnt natural dyes from training activities held in their villages by NGO workers. Kamolwan mentioned that NWD staff conducted natural dye training at the village hall. After that members had to practise and experiment themselves in order to search for suitable methods with the available local materials. Therefore, the knowledge captured from training activities, study tours and telling of villagers can be regarded as declarative knowledge for the same reasons as hand-weaving captured by observation. 
The narratives suggest that both knowledge of hand-weaving and natural dyes is associated with indigenous Isan knowledge. Indigenous knowledge is transferred with traditional methods-oral transmission, the teaching of senior community members and direct experience (Obomsawin, 2000; Hansen and VanFleet, 2003). In Prae Pun, members also transfer those knowledge areas to their daughters who become Prae Pun members. In a wider view, this study relates to the study of Abu-Rashed, Bertaux and Okunoye (2005) that societies in developing countries draw upon indigenous knowledge and culture for their economic development.

\subsubsection{Knowledge of community enterprise management}

CE management knowledge, on the other hand, is mainly derived from organisational routines based on situation of members, village culture, shared values and ethical principles. Knowledge of business management can be seen in the forms of procedures, experience and personal networks. This understanding is implicit and embedded in members, organisational routines and daily life rather than in documents and electronic formats. A good example is off-site selling practice which is decided on the basis of experience of office staff members. Wijittra commented that currently Prae Pun does not attend a general handicraft fair because the experience was not valuable, shows that knowledge of management is concerned with tacit and practical aspects. According to knowledge types described by Sanchez (1997), and Nahapiet and Ghoshal (1998), sets of experience in business management can be classified as procedural knowledge because they are derived from organisational routines.

Prae Pun's regulations and practice are based on shared values and ethical principles. Good examples are committee member recruitment and cloth quality standards. Criteria for committee member selection include trustworthiness and honesty. Meanwhile, Walai mentioned that cloth inspection teams cannot show favouritism towards their relatives. Furthermore, some activities, such as orientation and committee support programmes, are derived from the sense of community spirit and the sense of ownership. Lada and Sumalee explained that they have to train and guide new members to develop their skills at hand weaving in order to become full members. Both ethical principles and shared values can be viewed as theoretical according to the characteristics of knowledge described by Garud (1997) because they guide members’ performance. Lastly, the collaborative working style of Prae Pun 
involves personal knowledge creation. Prae Pun has a personal network where members can identify other members' abilities. Kunya stated that she sometimes asks members in other village groups to help her fix weaving problems if members in her village cannot. This finding is accordance with Skyrme (1999), and Van Den Bosch and Van Wijk (2001) that personal knowledge relates to the abilities of people in organisations.

In a word, knowledge of Prae Pun encompasses knowledge of traditional crafts and CE management which can be viewed as declarative, procedural, theoretical and personal knowledge. It is mainly tacit knowledge embedded in members, work processes and products, and derived from organisational routines, practice and observation.

\subsubsection{Financial resource}

Prae Pun has quite limited financial resources. The CE operates with capital from members' shares, its profits and support from NGOs and a customer (see section 6.2). The narratives of this study show that Prae Pun cannot earn much profit for enterprise development. This is supported by several members' statements, such as Raweewan, who said that the office should increase members' payments for every year, but has not done so for some time because Prae Pun has earned not sufficient profit. Meanwhile, Kannika mentioned that members have to try hard because they cannot hire a skilful manager or office staff members for their business operation and management.

\subsubsection{Information and communication technologies}

This study reveals that Prae Pun does not have much investment in information and communication technologies (ICTs). From my own observations, Prae Pun's office has only one desktop computer with common software for word-processing and internet services. It is used for document preparation and some communication with advisory committee members and support organisations. These documents include committee meeting reports, training and experimentation documents and sales documents. Only one office staff member and one advisory committee member who lives in Bangkok can operate the computer. In the village groups, none of the members use the computer for supporting their production, acquiring knowledge, 
sharing their experience or communication.

Members mainly communicate with each other face-to-face, rather than through telephones, mobile phones and computer systems. This is because they all live in the same or neighgbouring villages. From my observations, members simply visit each other if they want to share their work or request advice. Even though most members have mobile phones, they use them only for private maters and the signals are not clear. Prae Pun uses land line telephone and mobile phone for communication with members and local customers, and facsimile for communication with international customers. The manager normally goes in person to members' villages to explain special orders and discuss problems and solutions for cloth quality. Members from different villages normally communicate with each other face-to-face on cloth delivery days and at annual meetings, , training sessions, study tours and visits to villages. At the office, the four staff members work together in the small office, so can communicate with each other easily. Telephones are used for making appointments and follow up orders rather than for advice and sharing opinions.

\subsubsection{Social capital}

Significantly for this study, even though members have low levels of education, few skills in management and limited funding, Prae Pun possesses sufficient human and social capital to facilitate its business and group activities. As Prae Pun comprises seven village groups and its activities are conducted throughout all the groups, the CE has a wide network of social relations. Because of the committee systems and selfemployed systems, members have numerous opportunities to create social relations and network ties, such as on the job training activities and internship programmes. Raweewan stated that she asked members at another village to teach her how to make colour from stick lac. This shows that members trust each other which encourages willingness in sharing knowledge. Furthermore, as discussed in section 6.2, Prae Pun runs its business with the trust of each other and customers. This fits with the social capital theory suggested by Coleman (1988), and Nahapiet and Ghoshal (1998), because members are influenced to participate in Prae Pun activities with trustworthiness and mutual obligation.

Furthermore, this context also applies to the CE's relations with support organisations 
and customers, as mentioned in section 6.2. These findings can also be viewed as social capital (Coleman, 1988; Nahapiet and Ghoshal, 1998), because Prae Pun has a social network and social relation with support organisations and customers that provides values to generate actions and information.

Prae Pun relies on local resources including local knowledge, human resources, funding and social capital. However, ICTs are not much used for its businesses. Therefore, the findings on resource use accord with other findings, such as Haper (1985), Nelson (1987), and Halvorson-Quevedo (1991), that micro enterprises mainly do business based on local resources, skills, wisdom and appropriate, rather than advanced, technologies. Jommuang (1997) and Huntrakul (2000) have found that micro enterprises in Thailand have a high level of social capital which they utilise for business operations. In terms of financial resource, the findings of this research support Halvorson-Quevedo (1991), in that micro enterprises often have difficulty in financial terms, needing to rely on support from NGOs.

\subsection{Benefits}

The narratives of this study suggest that, for over a decade, Prae Pun has managed its business operations efficiently and effectively, and has provided members with many benefits, including income, business ownership and dividends, welfare to members and their families, and intangible benefits.

\subsubsection{Income}

According to the quota system, weaving members get around 2-3,000 baht or NZ\$80120 per month per person. The monthly income is an obvious benefit that leads to many women in members' villages wishing to join Prae Pun. Many members are able to afford good education for their children and meet their daily costs with this monthly income. Office staff members can earn income through salaries instead of weaving. The findings of this research match with other studiess, such as HalvorsonQuevedo (1991), Kitahara (2000) and Wijayaratna (2000), demonstrate that micro enterprises tend to provide the main income for population in developing countries. 


\subsubsection{Business ownership and dividends}

Prae Pun has made it much easier for rural women to own and manage their own businesses. Every member is an owner of Prae Pun. Members are also shareholders and receive dividends. They experience the feeling of being business owners, which is different from other cooperatives' members. As mentioned in section 6.5.2, members of Prae Pun are all borrowers together in the loan contract set up with Mr. K. The findings of this research reflect the characteristics of community enterprises discussed by several researchers including Waliasatian (1996) and Wasuntiwongse (1999) as being community owned. However, Prae Pun members have not often earned dividends because of limited sales and the need to use some income for business operations.

\subsubsection{Welfare to members and their families}

Prae Pun has enough money to provide its members with welfare programmes including loans, health insurance, and welfare for senior members and children of members. Prae Pun lends money from the organisation's bank account, in order to get more benefits from the funds, and provide opportunities for members to improve their quality of life and standard of living. This is beneficial as members are generally poor farmers, and hence would find it harder to obtain a loan from formal financial institutions. The findings of this research broadly support Halvorson-Quevedo (1991), who notes that being a part of an informal sector of micro enterprises may mean lack of support from more formal agencies. Currently, the Prae Pun loan fund has been registered as a credit union cooperative. Thus, members can deposit their savings and other members can borrow money. Prae Pun has provided membership loans with low interest rates, with the result, as Kannika explained, that many members own much more land, as well as bigger and better houses, and cattle.

Since weaving can create health problems, such as poor eyesight, pain, and dust allergies, Prae Pun's health insurance makes it easier for members to access health services without having to resort to limited government services The health insurance also covers members' families as well as themselves. Members pay 120 baht or around \$NZ5 /year and can claim up to 500 baht or around \$NZ20 /year for three members in their family. 
Prae Pun runs its business based on native knowledge which is handed down from the Isan ancestors. Prae Pun rewards these knowledge sources by providing welfare to senior members, who are considered the masters of Prae Pun, at the rate of 300 baht or \$NZ12 /month/person. Prae Pun also provides an education fund for students with three or four scholarships of 600-700 baht or around \$NZ25-29 per year.

\subsubsection{Intangible benefit}

Prae Pun also provides intangible benefits which include opportunities for members to expand their horizons and gain personal development and leadership skills through group activities and study tours. From my observations, most of Prae Pun's members, especially committee members, have self-confidence and leadership skills. They are accepted by their communities and play an important role in community development, such as a chairperson of Prae Pun, who is also a vice-leader of her village. Some committee members are proud of themselves because Prae Pun has grown to be wellknown CE with high quality cloth and truly management by its villagers.

Altogether, Prae Pun is a vital part of the local Isan community development, especially in terms of economic and gender development. Prae Pun also symbolises civil institution development, because members have developed themselves through Prae Pun activities and being a part of a grass-roots institution. This research supports Halvorson-Quevedo's (1992) findings, that micro enterprises allow women to show their potential in both family and economic issues, and improve the abilities of community members in problem solving and managing skills.

\subsection{Chapter summary}

In conclusion, Prae Pun is classified as a community enterprise that is fully operated by the community members. The 200 members are mainly rural women in seven villages of Khon Kaen province, Thailand. They are shareholders who feel they have full ownership of the business. The business is based on Isan culture and local resources-local materials, native knowledge and local wisdom. Even though its members have low level of education, Prae Pun is associated with several knowledge elements in the forms of indigenous knowledge and intellectual capital: human capital, customer capital and structure capital or social capital. Prae Pun has successfully designed its own operation and management systems with committees, 
self-employment, job sharing, open discussion and unique product offering. In terms of its village location and home-based production, Prae Pun has a culture of informality, socialisation and collaboration. The home-based system and unique product offering also involve interaction among members. Moreover, Prae Pun has a flat and less hierarchical management structure, and high commitment of members. These contexts involve many knowledge processes and learning processes, and provide supportive and incentive systems for its $\mathrm{KM}$, while, the marketing system also allows Prae Pun to obtain knowledge from customers and FTOs. Lastly, the CE's benefit systems serve as supportive incentive systems for members to develop their skills and search for knowledge. Chapter 7 will go on to discuss the knowledge processes of Prae Pun in detail, and chapter 8 will present a model to describe the overall KM of Prae Pun as influenced by the CE's unique characteristics and resources. 


\section{Chapter 7}

\section{Prae Pun's Knowledge Processes}

This chapter presents the findings of this research which focuses on the knowledge processes of Prae Pun which are the results of two months field work with narrative inquiry with 18 members of Prae Pun, observations of the researcher and document analysis. These show that Prae Pun has managed its knowledge through three main processes: basic skill creation, competence building and new knowledge creation. These phenomena will be used to create a knowledge management (KM) model to illustrate current KM practice in the next chapter.

\subsection{Prae Pun's knowledge processes}

Significantly for this study, Prae Pun does not have an explicit knowledge management system. However, community members undertake relevant activities that can be interpreted as knowledge processes. In this way, Prae Pun members focuses on competence development and knowledge acquisition through learning basic hand weaving from mothers, learning by themselves, training activities, study tours, document study, 'doing the job', local experts' involvement, and socialising with other weavers. The narratives in this research reveal that the KM of Prae Pun is primarily focused on creating organisational knowledge assets and organisational memories. Figure 7.1 shows the processes involved in the knowledge management of Prae Pun.

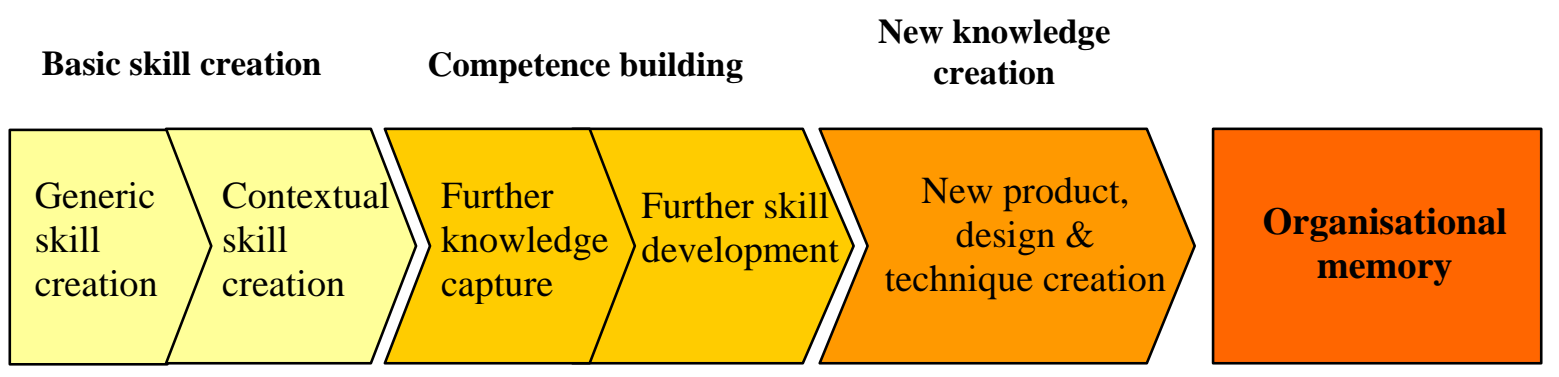

Figure 7.1 The knowledge processes of Prae Pun

\subsection{Basic skill creation}

As discussed in chapter 6, all Prae Pun members possess at least basic knowledge of hand weaving and some are already skilled weavers before they join the community. 
This is because traditionally, young Isan women learn hand weaving in the home in order to make cloth for their families. Even though they possess extensive knowledge of hand weaving before joining the CE, they need to obtain basic knowledge of Prae Pun's particular designs and techniques. This is because Prae Pun's modern cloth with traditional patterns and designs is different from the hand woven cloth traditionally made for personal consumption. Thus, members need to develop both generic and contextual weaving skills.

\subsubsection{Generic skill creation}

The narratives of this study suggest that, members learn basic hand weaving knowledge from their mothers or woman villagers. Moreover, they also capture basic knowledge of natural dyes and dyeing processes but this tends to focus on observations rather than the skill itself.

\subsubsection{Generic skills at hand weaving}

Previously, young women learnt how to weave with their mothers as teenagers in order to make cloth for their families. This is shown by several members' statements, such as Lada who mentioned that she learnt how to weave with her mother and her senior relatives when she was young in order to make cloth for their own consumption. This is because villagers used only hand woven fabrics at that time. Likewise, Kamolwan said that her mother taught her to weave kit in order to make kit pillows and mattresses for her brothers' upasampada ceremonies or ordination rites, and she also learnt how to weave mudmee to make simple skirts for herself. Mali stated that she learnt how to weave when she was 11 years old, when her mother asked her to weave cotton plain cloth. She then learnt how to make kit for making pillows.

Currently, even though hand weaving is less relevant to the villagers and young women who study and work outside their villages, the locals still use hand woven cloth for ceremonies and particular dresses. Indeed, I observed that villagers still use kit pillows for both family and ceremonial purposes, and wear simple mudmee skirts for particular events, such as merit-making events (Buddhist rituals, meditation, chanting and learning Dhamma) and festivals. Even though young women are less interested in hand weaving, the older Isan women still want them to learn. This is 
shown by Raweewan's statement that she needs to teach young people weaving and heddle shaft preparation because her eyesight is no longer good enough. Members who have daughters said they want their daughters to help them in their work and also to preserve Isan culture. Even members who have no daughters said they would teach them if they had. This situation extends to male members who have free time. One male member said he and other husbands learnt how to weave at home in order to help their wives. After the men have sufficient skills in hand weaving they can also become Prae Pun members.

Nowadays, young women normally take up weaving when they become mothers, and come back to visit or settle down in their home villages. Many members mentioned that they ask their daughters to help with weaving and teach them at the same time. Suwannee explained that she asked her daughter to help when she came back home for the birth of her baby. At the same time, she taught her every step of weaving. Now, after two or three years, her daughter has become a skilful weaver.

The narratives of this study suggest that obtaining generic skills at hand weaving involves the process of learning by doing under the supervision of mothers or female relatives. However, in order to weave skilfully, members need to practise. This is consistent with several members' statements. Kamolwan pointed out that she learnt how to weave from her mother, but could not do well at the beginning. She needed time to practise by observing examples of other members' weaving and reflecting on her own work processes. Lada also mentioned that she had to remember what her mother and senior relatives taught her, then practice on one or two looms in order to perfect basic skills, such as thread preparing, and plain cloth and loincloth weaving. After that she could learn other hand weaving cloth patterns, such as kit and mudmee, by practising at least one loom for each pattern. This is similar to Suwannee's remarks, that learners may be able to weave after completing only one loom but they become better weavers as they continue weaving.

Indeed, learning from mothers can provide more than just basic hand weaving knowledge. Daughters are able to learn as much as possible if they are interested. As shown by the narratives described previously, many members have already learnt the kit and mudmee patterns which require basic weaving skills. They started learning 
hand weaving with plain cloth and loin cloth, and after they have these basic skills, they can learn further hand weaving patterns. This is consistent with several senior members' statements that they can weave the same patterns as their mothers because they learnt from them. For instance, Kamolwan mentioned that she learnt all her weaving techniques, such as kit, mudmee and 4-kao weaving, in this way. In short, learning from mothers involves general skill development which may be only simple patterns or a range of varieties depending on mothers' knowledge and daughters' interest.

\subsubsection{Generic skills of natural dyes}

Learning from mothers is not limited to hand weaving. Daughters also learn about natural dyes from their mothers but this is learning by observation rather than by doing. This is because even though young women do not get involved, because the natural dyes stain their hands, the processes of dyeing often take place at their homes where they can observe those processes. Members indicate that they would like their daughters to help them in natural dye making. For example, Urai stated that natural dye making is quite a heavy task including the carrying of soaking wet cotton. Although reluctant to get involved in the actual dyeing process, the daughters learn basic skills in this way.

In fact, as with hand weaving, learning natural dyeing would not be hard for new members, if they wish to learn. This is consistent with many members' statements, such as Malee and Prarichat, who pointed out that learning hand weaving is not hard for them because they are familiar with weaving processes by having watched their mothers weaving daily since they were young. They readily possess declarative knowledge which they can utilise for procedural knowledge creation. The observations during this process can be viewed as declarative knowledge, according to Nahapiet and Ghoshal (1998), and Moorman and Miner (1998), because the daughters or new members have to combine such knowledge with practice in order to develop their skills. New members have to practise more hand weaving techniques and natural dyeing when they become members. Thus, new members could acquire generic skills in using natural dyes by doing together with their observations. Learning about natural dyes from watching mothers is similar to the findings of Garza 
and Ibbs (1992), who noted that observation is an effective knowledge acquisition method for a construction field.

Altogether, learning from mothers entails learning by doing, which involves generic skill development and observations. This is consistent with findings of many researchers including Verela et al (1995), Garud (1997), Sanchez (1997), Brown and Dugid (1998) and Lam (2000). They point out that procedural knowledge is derived from doing, practical experience and good practice over a period of time. Indeed, learning from mothers is associated with knowledge processes transfer. According to Carrillo, Robinson, Anumba and Bouchlaghem (2006), learning from mothers can be viewed as human-centred knowledge transfer, which focuses on learning processes and interaction. The narratives suggested that the process of learning from mothers and other women in villages comprises both processes of knowledge transfer: a communication and an interpretation process, as shown by the findings of Albino, Garavelli and Schiuma (1999). First, the teaching and advice of mothers can be viewed as the communication process because its allows knowledge to be transmitted from mothers to daughters. However, the communication process of Prae Pun focuses on face-to-face communication rather than an information system as suggested in the study of Albino, Garavelli and Schiuma (1999). The communication process of Prae Pun is effectivefor its knowledge transfer, because, according to Wathne, Roo and Von Krogh (1996), face-to-face communication is richest transmission channel. Second, the learners' practising, observing and reflecting on their own work are associated with the interpretation process. It can be seen that the daughters of Prae Pun members accept knowledge from their mothers, and adapt and apply it to their working styles. Furthermore, the transfer of knowledge from mothers also helps to preserve indigenous knowledge. This research broadly supports the finding of others, such as Sefa Dei, Hall and Rosenberg (2000), that indigenous knowledge is largely transferred from generation to generation by the teaching of senior community members.

\subsubsection{Contextual skill creation}

New members, even those who are skilful weavers, have to be oriented in order to obtain the contextual skills in weaving cloth for Prae Pun. These skills include the community's specific hand weaving and natural dyeing techniques. Prae Pun conducts 
several programmes for new members to create contextual skills. These activities are moderated employment programmes including orientation and trial period programmes, an internship programme, and a committee support programme. Prae Pun has moderated employment programmes for new and less-skilled members in both production and office work. The findings of this research broadly support the study of Wiig (1999) that training programmes and apprenticeship are effective methods for knowledge capture.

\subsubsection{Orientation and trial period}

Both new weavers and new office staff members have to be oriented and undergo a trial period for a while to learn about Prae Pun production and work processes and for their abilities to be observed. First, Prae Pun products are different from traditional cloth for family consumption. New members have to learn alongside skilful members how to meet Prae Pun cloth standards. The narratives of this study indicated that this process relies on peer assisted and informal systems. This is consistent with many members' statements. Prarichat and Kamolwan stated that new members should be trained in natural dyeing and taught how to weave for Prae Pun, because many processes related to products for sale, such as cloth winding, selvage and designs, are different from those intended for personal consumption. Sumaree explained that, currently, new members have to be interns supervised by skilful members until their cloth meets the required standards. Only then can they become a full member. Second, new office staff members work under the supervision of a manager and other staff members until they can perform their tasks by themselves. For example, Wijittra explained that she was in a trial period for three months, and initially, started working in the sales section with the supervision of the manager.

On this basis, orientation and training both contribute to contextual skill creation. New members and office staff members acquire necessary procedures and a certain level of skills, which constitute usable knowledge in order to perform Prae Pun activities through doing and practice. These findings match with the concept of procedural knowledge discussed by Ryle (1949), Verela et al (1995), Sanchez (1997), and Brown and Duguid (1998), which is derived from practical engagement in order to obtain knowledge for performing tasks. 
As with learning in the home, these activities also comprise a process of knowledge transfer and knowledge utilisation. The narratives indicate that the orientation and trial period programmes provide channels for skilful members to transfer their knowledge to new members. As with learning from mothers, training by skilful members of new members can be viewed as a rich channel for knowledge transfer because it involves face-to-face communication. Furthermore, according to the concept of effectiveness of knowledge transfer introduced by Cohen and Levinthal (1990), and Gupta and Govindarajan (2000b), training by skilful members has a high absorptive capacity because new members already have a basic knowledge of hand weaving and are, therefore, familiar with receiving knowledge. Moreover, both parties have similar attributes, interests and culture, which also enhances knowledge transfer. These processes are similar to the knowledge transfer described by Collison and Parcell's (2004) study, in which peer assistants distributed organisational knowledge to non-skilful organisational members. For knowledge utilisation, the narratives showed that new members are encouraged to apply knowledge from these activities to meet the requirements of cloth production that enable them to become full members. These findings represent knowledge utilisation in terms of the demand driven approach of Swan, Scarbrough and Hislop (1999), and the operational aspect of Zack (2002).

\subsubsection{Internship programme}

Prae Pun's office also has an internship programme to develop contextual skills for members who may become new office staff members. Penchun explained that the office requires members to be interns at the office for two months with an allowance. Prae Pun prefers members or members' daughters to be office staff members rather than external people, because they better understand Prae Pun's work. This programme allows members to learn office work and obtain contextual skills at business operation. After passing the internship programme, the members can be office staff members. As with the trial period and orientation programmes for new weavers, the office internship programme is another moderated employment programme that fosters skill creation, knowledge transfer and knowledge utilisation. 


\subsubsection{Committee support programme}

Another aspect of contextual skill creation involves committee support programmes. In the village groups, committee members have to support community members, especially new members, in developing hand weaving techniques in order to meet cloth standards. This programme is similar to the orientation programmes, but the committee support occurs throughout the period of membership. Committee members regularly visit members, especially when they have requests or messages from the office or the board committee. This is illustrated by some committee members' statements. Lada stated that she often visits members in her village to suggest weaving techniques and designs in order to ensure that their cloth meet standards. The narratives of this research revealed that even senior members seek information from committee members, especially how to make cloth to meet specific customer preferences which committee members obtain from the office. Pitsamai and Kunyanew committee members - explained that they have to visit members in their villages to observe their situations and support them by sharing information from the office and commenting on their weaving, even though they are younger than some members.

As with the orientation and internship programmes, the committee support programme allows members to capture contextual knowledge as part of knowledge transfer and utilisation. This activity also generates a rich transmission channel for knowledge transfer from committee members to new members, enabling members to undergo the process of knowledge interpretation through practising and seeking clarification. Furthermore, this activity also contributes to knowledge utilisation through a demand-driven approach and operational aspect because members are willing to ask for and apply the suggestions of committee members to their work (see section 7.2.2.1).

Altogether, basic skill development focuses on new members acquiring generic skills and contextual skills in order to work for Prae Pun. Both generic and contextual skill creation focuses on learning by doing and observation. This involves several knowledge processes including knowledge creation, knowledge capture, knowledge transfer and knowledge utilisation. Significantly for this study, the teaching can be viewed as indigenous knowledge transfer systems in which traditional knowledge is transferred from generation to generation-from mothers to daughters and from 
skilful members to new members. For business operation and management knowledge, Prae Pun provides contextual skill development for new office staff members, which also focuses on learning by doing. After becoming members with basic skills, Prae Pun also pays attention to expanding members' capabilities in order to develop the community's businesses. These processes will be discussed in the next sections.

\subsection{Competence building}

The narratives of this study indicated that Prae Pun pays close attention to educational activities both group and individual activities in order to develop members' competencies. Even though they are skilful at hand weaving and natural dyes, members still need further knowledge in order to improve the quality of cloth and produce a range of varieties of cloth to meet customer preferences and marketing strategies. Many members stated that they are still not good weavers, and need to acquire further knowledge of both hand weaving and natural dyes. This is shown by several members' statements. Onanong said she thinks that she is able to weave but is not a good weaver yet. She needs more practice and considers that weaving is lifelong learning. For natural dyes, most members stated that they have a lot to learn. Malee commented that natural dyes still fade easily. Meanwhile, Kamolwan observed that most colours Prae Pun makes are earth tones, and she felt that Prae Pun needs to develop more techniques to make longer lasting colours and more varied colours. In these ways, the competence building of Prae Pun can be seen to focus on further knowledge capture, particular technique capture and further skill development.

\subsubsection{Further knowledge capture}

Further knowledge capture of Prae Pun is associated with both internal and external acquisition. Initially, Prae Pun focused solely on external knowledge capture in order to widen the knowledge and world view of members. This is because they had less experience and knowledge of making cloth for sale and managing a business. As mentioned in the previous chapter, all community members and office staff members are rural villagers who are not familiar with modern textile and marketing practices. From my own observations, the villagers do not use the modern Prae Pun place mats, table cloths and rugs themselves. In terms of colours, most hand woven cloth used by villagers and sold in local markets tends to be bright and in contrasting shades. 
Therefore, members needed to capture further knowledge in order to work for Prae Pun. The external knowledge capture has largely occurred through study tours, training activities, document study and expert involvement. Currently, Prae Pun focuses on capturing Prae Pun knowledge from skilful members to new members through its working processes. This is because Prae Pun has gained a range of skills and experience over 10 years, but lacks the budget to organise regular study tours and formal training activities.

\subsubsection{Study tours and sharing forums}

Prae Pun conducted several study tours, especially in the initial period, visiting other weaving communities, in order to provide opportunities for members to learn about hand woven cloth patterns and designs in order to develop their competencies to make cloth for Prae Pun. Many members, such as Prarichat, Onanong and Malai, mentioned that they visited many hand weaving groups in other provinces and overseas during the time when NWD worked with Prae Pun. Currently, Prae Pun still conducts study tours at least once a year but mainly only for committee members because of limited budget.

Study tours allow members to explore a variety of cloth patterns and designs and the weaving techniques of other hand weaving groups in order to generate ideas to create their own products and improve their quality of cloth. This is consistent with several members' statements, such as Suwanee and Malai who mentioned that they had obtained several mudmee and tine jong designs from study tours, which they could then apply to make kit designs for Prae Pun products. Similarly, Kannika observed that Prae Pun has more than just the common Isan kit designs because members adapt other pattern designs to create new kit designs. In fact, kit is a different cloth pattern from mudmee and tine jong, and uses different weaving techniques (see appendix E). In order to adapt any cloth pattern to make kit designs, members have to engage in creativity and experimentation. Raweewan also stated that members obtained their jug jig design, which is a float pattern design, from a study tour. Subsequently, members in the Huafai groups apply it to make big blankets and rugs. However, members cannot apply their observations directly to their work. They need to search for the particular procedures, develop the skills and verify their observations (see section 7.3.2.1). 
Prae Pun also organises study tours for office staff and committee members to expand their experience in business management. Some committee members, such as Napaporn and Raweewan, mentioned that they visited several handicraft groups in order to learn about their enterprise management. As with production knowledge, experience and observations of business management cannot be applied directly to Prae Pun management systems. Raweewan observed that Prae Pun may not operate the same as other handicraft groups, because Prae Pun has different situations.

The narratives of this study revealed that study tours have enabled members to capture external knowledge, which is concerned with fact and observations. As discussed in section 7.2.1, this can be interpreted as declarative knowledge and this process as knowledge capture by observation. Significantly, most members found that the experience and observations from study tours require them to engage in verification, adaptation and practice in order to create the particular procedures appropriate for Prae Pun (see section 7.3.2). These findings match with the general comments of Bhatt (2000) who points out that organisations have to evaluate and adapt knowledge from outside because it cannot be directly replicated.

However, not every member can attend study tours and Prae Pun cannot afford for every member to go on a study tour at the same time. Some members state that they cannot leave home as they have to do farm work and take care of families. The narratives of this study indicated that particularly young mothers with children and senior members are uncomfortable with travelling. Kamolwan said that she was worried about her daughter when she had to stay overnight on study tours. Meanwhile, Mali-a senior member — stated that she became sick when she had to travel away from home. For this reason, Prae Pun always conducts sharing forums for passing on knowledge from the study tours to members who have no chance to join the programmes. Thus, sharing forums allow all members to capture external knowledge. From my own observations, a sharing forum takes the form of discussion. Members not only tell what they learnt from the study tours, but they also discuss and criticise the acquired knowledge. Both study tour participants and non-participants compare that knowledge with the situations in their villages and Prae Pun. In this way, they judge the appropriateness of captured external knowledge based on their previous experience. 
Therefore, the sharing forums involve knowledge capture and verification. This research supports the findings of Hylko (2005), in that organisational members can capture knowledge through discussion. The sharing forums can be also viewed as knowledge verification. As suggested in the studies of Weick (1979), Bhatt (2001), and Collison and Parcell (2004), knowledge can be confirmed through debating from the different perspectives of participants' particular villages, known as knowledge verification by critique.

\subsubsection{Training activities}

Prae Pun has also conducted several training activities for members to capture further knowledge, especially during the initial phases of Prae Pun. Prarichat and Lada explained that NWD regularly conducted several training activities for members. Each year focused on different topics, such as cloth design, cloth quality and equipment selection. Initially, the activities concentrated on formal training by external resource persons to provide information in all areas in order to expand members' experience, particularly in relation to natural dyes and cloth designs. This is because villagers were inexperienced in these areas (see section 7.3.1). For example, the villagers stopped using natural dyes a long time ago. Walai mentioned that her grandmother used to make a black colour from makleo, which is an ebony fruit, but currently no villager makes natural colours. Members mainly learn about natural dyes from NGO workers. This is supported by many members' statements, such as Kamolwan, who said that, at the beginning, Mae Al (an NWD staff member) gave them training on natural dyes at the community hall. Raweewan and Mali also mentioned that they had attended a training programme on natural dyeing with NWD. Many members, such as Mali and Kamolwan, acknowledged that the natural dyeing learnt from NWD is the same method as their mothers and senior villagers used, but there are now techniques for making more beautiful, and more long-lasting colours. Kamolwan also said that her senior relative mentioned that she used to make natural dyes with the same method as in the training.

The narratives of this research suggest that training activities allow Prae Pun members to both capture knowledge from external sources and retain their experience. However, members cannot directly apply both captured knowledge and retained knowledge to their work. As with knowledge gained from study tours, they need to 
review and practise in order to find appropriate techniques for their situations and to refine their skills. This is shown by several members' statements. Napaporn observed that she made natural dyes with the method she learnt in the training activities but could not achieve the same results. She recognised that some materials are different. For instance, her village does not have a deep well containing rusty water, as used in the initial training session. Meanwhile, Raweewan commented that members in her village use mud and makleo to make dark colours rather than black as her seniors did. In terms of the dyeing process, members now have to take mud from the paddy field to their homes and soak the cloth for only a few hours instead of leaving it overnight in the paddy field. This is because there are no longer any buffalo mires or mud ponds, and working at home is more convenient for observing the results. These examples show how members have captured external knowledge and applied it to their own situation.

In addition to weaving and dyeing skills, members also obtain further knowledge of pattern design and business management from training activities. Many members, such as Lada and Kamolwan, mentioned that they had attended a training programme in order to learn how to design cloth to meet customer preferences. Meanwhile, Raweewan stated that she undertook a training programme on modern product design with ThaiCraft-a FTO. Parichat also mentioned that she has participated in several training activities, such as cloth design, cloth quality and group management conducted by NWD. She remarked that most training activities mainly take the form of workshops and seminars, which comprise demonstrations, simple practice sessions and sharing forums. She observed that the facilitators do not know some topics well because they are unfamiliar with the Prae Pun context. Members have to share their situations with the resource providers in order to obtain full understanding and the right information. This confirms the findings of Hylko (2005) that discussion facilitates knowledge capture. The training activities of Prae Pun are also in accordance with knowledge verification, according to Weick (1979), Bhatt (2001), and Collison and Parcell (2004) because the training activities involve debate and criticism. However, as with knowledge captured from study tours, knowledge captured from training needs practical engagement in order to be fully understood. Prarichat also noted that some programmes require a period of time for practice, such as particular cloth design with harmonious colours (see section 7.3.2.1). 
The narratives suggest that, as with study tours, training activities allow members to capture further declarative knowledge. However, practice is necessary in order to create procedural knowledge. In a sense, practical engagement is concerned with usable knowledge which must be reviewed and adapted to suit each village situation in order for members to develop full competence to work for Prae Pun (see section 7.3.2).

\subsubsection{Working}

Members also build competence by capturing further knowledge from other members, villagers and customers while they are working in the community's collaborative, informal village environments that allow them to socialise and capture knowledge from each other.

\section{Collaborative working environment}

Both the production units and office can be defined as collaborative, according to the concept outlined by Gray (1998), because members and staff members work together in order to complete particular jobs. Each environment allows members to share their experience and capture knowledge from each other. In village groups, members prefer to work together in groups of three to four neighbours, while some tasks actually require group-work. Raweewan mentioned that people prefer to work together, especially when they have to make natural dyes for specific orders in order to ensure the same colours for the entire batch. Furthermore, she explained that she has to weave big blankets with her grandmother. Such weaving requires two weavers working together because the blankets are made with wide reeds. Lada mentioned she can explain to new members how to make natural dyes when they work together. The narratives suggest that skilful members take the opportunity to share and transfer their knowledge, especially tacit knowledge, while working in collaboration with others.

As with cloth production, the operational and office work is also collaborative. As discussed in chapter 6 , the office has a limited number of staff members. They have to operate the activities as a job sharing system where staff members work and take responsibilities together. This environment involves interaction and sharing experience among office staff members. From my own observations, all office staff members had to prepare cloth for shipping to a handicraft fair and two of them took 
turns to work at the fair. Some other activities, such as wholesales at the office, cloth inspections, and group and committee meetings, also enable many staff members and members from the village groups to work together and share their opinions at the same time. A good example is the cloth inspection activities. Napaporn mentioned that she would often ask members from other village groups how to make a particular cloth if she saw their cloth was beautiful or looked different from hers. Thus, operation work also provides opportunities for capturing further knowledge from members in different villages groups.

The narratives suggest that both production and operation work is collaborative, allowing members and staff members to share and capture further knowledge from skilful members in order to develop their competencies. This research supports the findings of Sveiby and Simons (2002) in that a collaborative environment tends to enhance the willingness of organisational members to share knowledge. The knowledge capture through working at Prae Pun can also be viewed as learning from others, according to the knowledge acquisition model of Ryu et al. (2005). Moreover, as noted by Wathne, Roos and Von Krogh (1996), Cohen and Levinthal (1990), and Gupta and Govindarajan (2000b), face-to-face communication and the absorptive capacity of old and new information serve to enhance knowledge transfer (see section 7.2.2.1).

\section{Informal and village environment}

Both the production units and office can be regarded as informal working environments. The home-based production involves informality as members work according to their village culture. Meanwhile, the office staff members also have a working style like villagers rather than a corporate environment. The informal and village environments allow members to engage in several activities leading to further knowledge capture.

First, at village groups, members can ask their daughters to help weave, and teach them at the same time. This is consistent with several members' statements, such as Urai and Suwannee, who mentioned that they often ask their daughters to weave for them when they are busy. This shows that members work according to the village culture where family and work relationships are intertwined. Meanwhile, Mali stated 
that some young villagers call her for guidance with their weaving at their places while she is weaving at home. If she can, she will go and help them, or at least offer suggestions if she is busy. As discussed in section 7.2.1, such informal teaching by mothers and women villagers is a process of knowledge transfer.

Second, working within the village culture involves socialisation and knowledge sharing among members. From my own observations, members like to visit other members when they have a break from weaving, in order to share their situations and help each other solve problems. This is supported by Onanong, who said that skilful members in her village showed her how to weave improperly dyed silk in such as way as to make proper coloured cloth. Furthermore, some members visit others on private occasions, such as when members have merit making events and traditional ceremonies in their villages, which also generates further knowledge sharing and capture. One member mentioned that she is obligated to be a real friend or 'siew' with a member in another village. Traditionally, Isan society has a custom in which people make a commitment with someone they feel they can get along with in the 'Pook Siew' or a real friend declaration ceremony (Buasri, 1983). Through a blessing ceremony or 'Su Khwan', a village spirit master invites the gods to bless and take care of the couple, and their friends bless them by tying tight or 'pook' cotton thread around their wrists (Baan Maha Community, 2006). After they become siew, they will visit and help each other, and may work together (Kritsanaphathi, 1983). Indeed, such a relationship serves to enhance knowledge sharing and capture even beyond the immediate village group.

Third, the home-based production system allows members to capture further knowledge from people in their villages. The narratives of this study reveal that villagers often visit Prae Pun members at their work places and share their experience with them. This is supported by Kamolwan's comment, which noted that senior villagers in her village observe her natural dye making and comment that her method is the same as in their time. Such socialisation with villagers contributes to local knowledge transfer and capture. These findings fit with indigenous knowledge systems discussed by Sefa Dei, Hall and Rosenberg (2000), and Obomsawin (2000) in that information is transferred by oral transmission and from elder community 
members. However, methods used in a senior villagers' period might not always be suitable for the current situation

Furthermore, members also obtain cloth designs from the villagers. Many members, such as Napaporn, Raweewan and Kamolwan, stated that they weave cloth using designs that they have found in their villages. Malee also explained that she carefully observes the designs of cloth from any events in her village. Then, she can borrow them when she makes cloth. As with knowledge captured from villagers, members need to adapt any such designs to match with Prae Pun situations. This is because customer preferences are different from villager preferences, as emphasised in several members' statements, such as Onanong and Kamolwan, who pointed out that customers do not like designs, such as tree, as the villagers do. They prefer small designs and animal designs, such as elephant and keelean (Qilin, an animal in fiction). This process be viewed as knowledge verification because members utilise and observing its reflection from the market. This research supports Collison and Parcell's (2004) findings, that knowledge can best be verified through being applied to work processes and reviewed by organisational members.

Fourth, managing work at Prae Pun is also informal. Committee meetings normally take place at the office in a relaxed manner and are also held in the villages. From my own observations, there are many opportunities for informal sharing during committee meetings. The environment allows committee members to freely share their knowledge with each other. Lastly, the informal working environment within the office also involves socialisation among office staff members, and between sales staff members and customers. Office staff members work together as part of their of job sharing but they also eat lunch and some even stay overnight together at the office. Sales staff members often have informal interactions with customers, especially longterm customers. Therefore, office staff members have opportunities to capture further information amongst themselves and knowledge about customer preferences from customers. According to the concept of social capital outlined by Coleman (1988) and Stewart (1997), this socialisation can be seen in terms of customer capital. This is because the interaction between sales staff members and customers provides channels and information to facilitate Prae Pun activities, especially cloth production. Many 
members mentioned that they often request sample designs from the office because the office staff members know customer preferences.

In short, working within the village culture and informal environments entail social interaction and social relations. These environments allow skilful members to transfer knowledge to less-skilful members, as well as sharing knowledge with villagers and customers. This research supports the findings of others, such as Scarbrough and Carter (2000), Ford and Chan (2003), and Mitchell (2005), that informal interaction is an important way for organisational members to share knowledge with each other.

Therefore, working within collaborative, informal and village environment enable members and office staff members to capture further knowledge, especially tacit knowledge from skilful members, other villagers and customers. These contexts lead to members' competence development. The knowledge captured from members is more practical than that from villagers and customers because members are in similar situations. They share experience that is verified through their working. On the other hand, knowledge captured from other villagers tends to be facts about their situations. It is necessary for members to apply this knowledge in practice in order to verify its appropriateness and acquire the exact procedures for current situations. Experience shared from customers is declarative knowledge which members also have to verify in terms of its appropriateness for exact procedures. Therefore, Prae Pun's collaborative and informal working environment can be viewed as facilitating further knowledge capture by learning from others, as noted by Ryu et al. (2005), and discussed previously in this section.

\subsubsection{Document study}

Members also obtain further knowledge from written sources, even though, as rural farmers, they are not familiar with documentation. These documents include designs from the training activities and formal Prae Pun documents. Most training documents focus on traditional Isan cloth designs and have been provided by NWD staff members. Suwannee showed me documents of pattern designs that she received from a training activity. She uses them for mudmee cloth design and also for kit design. Kamolwan also showed me her notebook with some designs that she saw on study tours and notes about natural dyes. She also has many copies of mudmee designs from 
NGO workers but rarely opens her notebook because she can remember the designs after making them once.

Prae Pun's formal documents include activity and experimentation reports, committee meeting reports, and sales documents. Activity reports, such as experimentation and training reports, contain information about particular production techniques. Meanwhile, the committee reports mainly comprise group and business management strategies and regulations. This is consistent with Napaporn's statement. She explained that the office gives copies of the committee reports to new committee members so they can learn about Prae Pun. Any members can also ask for these reports from the office if they are interested. According to Garza and Ibbs (1992), reviewing organisational documents is an effective method for organisational knowledge acquisition. Unfortunately, no new committee members mentioned that they had studied committee reports. The narratives of this study reveal that they learn about Prae Pun management systems through working instead. Walai remarked that new committee members tend to already know Prae Pun's management systems, because committee members and members work together. Meanwhile, sales documents, such as specific customer orders, contain cloth patterns, details of designs and directions how to make them, which some members have to study to complete the orders. On this basis, document study can be described as competence development of members, mainly in the area of cloth design rather than operation and management.

The documentation of Prae Pun can be viewed as organisational memories, according to the concept discussed by Walsh and Ungson (1991), and Nilakanta, Miller and Zhu (2006), The documents also represent knowledge transfer from generation to generation, according to Hansen, Nohria and Tierney (1999), and can be classified as the tool-centred approach of knowledge transfer, even though this is not an effective method for Prae Pun, because most members do not access the records.

Altogether, further knowledge capture at Prae Pun occurs through study tours and sharing forums, training activities, working and document study. The process mainly entails declarative knowledge which is a crucial resource for procedural knowledge creation-further technique acquisition and new knowledge creation. In this way, the 
findings of this research support Nonaka's (1994) organisational knowledge creation model that includes the process of individual knowledge enlargement.

\subsubsection{Capture of particular techniques}

Particular technique capture is another aspect to develop members' competence. As discussed in the previous section, after members capture declarative knowledge, they need to search for procedures that are suitable for their situations. The narratives of this study reveal that capture of particular techniques tends to focus on practical activities including experimentation, on-the-job training and the involvement of local experts.

\subsubsection{Experimentation}

Experimentation enables members to apply the particular procedures of knowledge captured from observations. The narratives of this study suggest that Prae Pun members are enthusiastic experimenters. They often conduct their own experiments in order to search for exact procedures and full understanding of their observations from study tours and trainings activities. Individual experimentation comprises the trial and error method. As discussed in section 7.3.1.1, members first saw the jug jig design on a study tour, then, one senior member undertook a period of experimentation in order to determine the exact heddle shaft configuration for the design.

For natural dyes, many members state that they also have to conduct experimentation in order to develop techniques that are suitable for the Prae Pun environment. Napaporn commented that she also took a period of time to search for the methods to make natural dyes in the same colours as she had learnt from a training activity. Finally, she recognised that some materials in her village are different from those at the training, so she had to experiment to make the colours using other procedures. Meanwhile, Raweewan explained that, instead of soaking cloth in mud overnight in the paddy fields to make black as in the past, members have now found that they need soak cotton for only few hours in order to make dark colours. According to Ryles (1949), Verela et al (1995), and Sanchez (1997), such individual experimentation is a method of procedural knowledge creation because members combine declarative knowledge and previous experience together with practice and learning by doing. This learning by doing is also similar Ryu et al.'s (2005) concept of knowledge acquisition 
According to the findings of Holsapple and Joshi (1999), and Zack (2002), the findings of this research can be viewed as knowledge utilisation through the demanddriven approach and the operational aspect, because members use their experience and observations from study tours and training activities to create their own procedures. Furthermore, these processes can also be viewed as knowledge verification and adaptation as outlined by Choo (1998) and Collison and Parcell (2004), in members' application of knowledge and testing the results

\subsubsection{In-house training activities}

The narratives of this study reveal that, recently, members mainly have attended inhouse training activities rather than study tours, in order to acquire particular techniques. The activities focus on 'on the job' training conducted in each village group or individual training sessions where members who are interested to learn particular techniques request skilful members to teach them. This is consistent with several members' statements, such as Kunya, who mentioned that she asked her neighbour who is a Prae Pun member to teach her how to weave thick mats with 3kao technique. Her neighbour gave her guidelines at her weaving place.

Sometimes, on the job training occurs after study tours in order to train other members who do not have the opportunity to attend. Raweewan stated that members learnt how to use the 'tong leang' heddle shaft on a study tour to Roi-et province. After that one member taught others in this village, with supervision from the resource person. Tong leang is a particular heddle shaft used to prepare kit designs. The narrative suggests that members, especially the resource person, can quickly capture this knowledge as they already have experience in kit making. The findings of this study support the findings of Gupta and Govindarajan (200b) on the effectiveness of knowledge transfer (see section 7.2.2).

Therefore, the narratives suggest that on the job training at Prae Pun is an example of procedural knowledge acquisition. Trainees can learn procedures and certain levels of skills in order to perform activities. Like the community's moderated employment programmes, in-house training activities contribute to members' knowledge transfer and knowledge utilisation (see section 7.2.2.1). 


\subsubsection{Local expert involvement}

Prae Pun also builds its competencies by inviting local experts, in order to have a variety of skills and abilities in making cloth. Kamolwan mentioned that one member in her village asked her to join Prae Pun, because the CE did not have many members with her skill in mudmee at that time. This finding is similar to the practice of large enterprises and intensive knowledge organisations recruiting people with particular skills and abilities (Starbuck, 1992; Gupta, Sharma and Hsu, 2004). From my own observations, the expert involvement includes the staff from local support organisations. Thai Craft—a fair trade organisation—sends some staff members to help Prae Pun design products for particular occasions. The above data suggest that expert involvement provides vital competence creation that allows Prae Pun to acquire procedural knowledge—skills and experience—-possessed by skilful members and resource people.

Prae Pun captures its particular techniques through experimentation, in-house training activities and expert involvement. These techniques can be viewed as usable knowledge for activity performing. They comprise procedural knowledge which is derived from practical engagement and verified in terms of its appropriateness for the Prae Pun environment. Most significantly, for this study, the processes of acquiring the particular techniques involve knowledge utilisation and verification by applying, testing and reviewing the results,

\subsubsection{Further skill development}

All Prae Pun's units—-production, office and committees—need to develop their skills in order to build their competence to work for the CE.

\subsubsection{Production skill development}

Besides attending educational activities conducted by Prae Pun, members continually seek to increase their competencies by practising and developing their skills when working and in their free time. As mentioned in section 7.3.1.3, members work without time limits and can switch to private work any time. From my own observations, members have a high commitment to improve themselves for the good of Prae Pun all the time. This is consistent with Onanong's comment that she is not a good weaver. She mentioned that she still needs to practise on hand weaving and 
natural dyes. Moreover, she always does silk preparation when she has free time. Pitsamai also explained that her grandmother taught her all processes of cotton and silk bleaching, but she still needed to practise for a period of time in order to develop her skills. She explains that good bleaching can be measured by checking the texture of the threads by hand. Another good example of skill development is the harmonious colour cloth design of Prarichat. She mentioned that she took a period of time to develop the harmonious colour design that she learnt from a training activity, by designing on her own. Kannika also observed that members took some time before they accepted the new colour concept and began to design cloth with harmonious tones themselves.

The narratives suggest that the more members work and practise, the better their skills development. This is supported by several members who said that they do not need to open their notebooks for most processes of working because they know how to do them once they have applied the knowledge in practice. As mentioned in section 7.3.1.4, Kamolwan could prepare weft for particular designs of mudmee without opening her notebook after she had done it for a while. Meanwhile, some members, such as Napaporn and Kamolwan, also commented that they do not refer to documents when they make natural dyes. These findings fit with Ryle's (1949) view, that skills can be derived through good practice.

\subsubsection{Business operation and management skill development}

As with the weaving members, office staff and committee members have to develop their management skills because they have little experience in business operation and management. They have to pay close attention to learning and practice while they are working in order to capture business and group management knowledge.

First, office staff members have put a lot of effort to build their capabilities in business operation. Kannika —an advisory committee member-observed that members have to practise for two years in order to be competent in business operation. From my own observations, currently, one sales staff member works well on sales and stock-keeping. She can complete sales transactions and maintain the stocks in the showroom. Meanwhile, another office staff member who handles international orders is not good at English communication, but she can deal with 
international customers by herself after being supported by volunteers for a period of time. She also displayed knowledge of handicraft fairs, mentioning that, currently, Prae Pun does not attend any general fairs because members do not have experience selling at such occasions. Nevertheless, these narratives suggest that office staff members who are rural villagers can develop a range of business operation skills through working.

Second, members in the village groups also have opportunities to obtain business operation skills. These members have to perform some tasks without pay as part of their Prae Pun duties. Every village group has a cloth standard committee to inspect the cloth and every member has to deliver cloth. Walai explained that a cloth inspection team has three people. Two people check the fabrics and another person records. One copy of the records is given to the office and another copy is kept at the village group for payment after delivery. As with production, in this way members can develop their skills at business operation and documentation while working.

Third, the narratives of this study indicate that committee members become the managerial team without prior management skills and experience, which they obtain simply by performing their duties. These skills include problem finding and solving, critical thinking, and group and business management. This is consistent with some members' statements, such as Pitsamai (a new committee member), who remarked that she was selected to be a committee member immediately when she became a member solely because she was good at hand weaving, skilful at documentation and available to travel for committee work, rather than for her management skills. Kannika said she believed that committee members developed through critical thinking. Thus, advisory committee members try to give them information and stimulate them to express ideas, discuss and make decisions for managing Prae Pun by themselves. From my own observations, a committee meeting involves a lot of discussion in order to find solutions for particular problems. Even though new committee members are not familiar with expressing their ideas, as they are younger and less experienced than skilful committee members, they are in a learning process. This is consistent with Napaporn's statement that, initially, she was afraid of the hard discussion of committee meetings, but now it is familiar and she thinks the management of Prae Pun needs open discussion in order to find group solutions. 
The narratives of this study reveal that Prae Pun office staff members, members and committee members have some certain skills in business operation and management. They can properly deal with business functions and have managed Prae Pun for over 10 years. This is consistent with Prarichat's statement that Prae Pun was able to maintain its business during the economic crisis period whereas many other community enterprises collapsed. From my own observations, currently, most Prae Pun members, especially long-term members, are confident. This is consistent with the statements of Kannika and Malee. Kannika mentioned that initially members were not confident, but that members became confident after two years of working because they could buy their building. Malee also stated that initially, she was not confident to talk with customers because she could talk only the same as the rural villagers. During my research, however, compared to other members, she could talk with me without being shy. This self-assurance is also apparent in long-term office staff members. They were confident to share their experience in working with Prae Pun with me. Meanwhile, a newer office staff member was shy to talk to me. The narratives suggest that working over a period of time enables members to develop their skills and gain confidence. Therefore, further skill development of Prae Pun occurs through the activities of members themselves. This supports the findings of several researchers including Nonaka (1994) and Lam (2000) that skills_procedural knowledge—is derived through doing.

In these ways, competence building for Prae Pun is concerned with both declarative knowledge capture to enlarge members' experience and observations, and usable knowledge - particular techniques and skills to enable members to work and develop the businesses.

\subsection{New knowledge creation}

Because of the community's unique product offerings and cloth standards, members are encouraged to create new designs and new products and search for new techniques. The new knowledge creation of Prae Pun occurs through experimentation, working and serendipitous discovery. 


\subsubsection{Experimentation}

As mentioned in section 7.3.2.1, Prae Pun members are experimenters. They often conduct experiments while working or when they have free time. The experimentation does not involve only particular techniques, but it also involves new knowledge creation. Furthermore, at the organisational level, Prae Pun also conducts group experimentation in order to search for new techniques.

\subsubsection{Individual experimentation}

Members' individual experimentation focuses on the trial and error method and involves the creation of new techniques and new designs rather than new products. Members often search for natural dyes from new materials by themselves through trial and error. This is consistent with several members' statements, such as Pitsamai, who said she often tries to make natural dyes with any materials that she thinks could give beautiful colours. However, she does not keep a record of her experiments, so she often cannot reproduce the exact colours a second time. She needs to continue testing for a while in order to perfect the methods.

Members also often experiment in terms of weaving techniques. Prarichat mentioned that members search for weaving techniques to make cloth to meet Prae Pun cloth standards. She had to try and observe how to make the good selvage of cloth while she was weaving. Kamolwan said she also discovered the technique of cloth winding while weaving, because she realised that she could not wind woven cloth the same way as cloth for personal consumption. The narratives suggest that members have discovered particular techniques which can be seen as usable knowledge for Prae Pun. Members who find a particular technique always share their discoveries with colleagues through socialisation and particular activities, such as group meetings and cloth delivery. This is consistent with Raweewan's comment that members always tell each other what they know about techniques.

The narratives in this study suggest that new designs come about as a result of creativity and trial and error methods in order to search for exact procedures. As mentioned in section 7.3.1.1, members had an idea about how to apply the designs of other cloth patterns to make kit designs. Then, they conducted experimentation to search for particular methods. Suwannee explained that she studied other cloth 
patterns and tried to make them with a kit pattern technique. Furthermore, members also generate new designs without any patterns through the same method. Raweewan explained that she also draws new designs on paper and makes them up using the trial and error method. If the results are not the same as her ideas, she will try them again.

In short, Prae Pun engages in individual experimentation focusing on new techniques and new designs, which involves creativity and trial and error in order to create usable knowledge. The findings of this research are in accordance with Nonaka's (1994) view, that the trial and error method entails tacit knowledge creation. According to procedural knowledge creation introduced by several researchers as discussed in section 7.2.1, the individual experimentation can be viewed as a process of learning by doing in particular situations.

\subsubsection{Group experimentation}

The group experimentation among community members is focused on seeking out new varieties of colours and alternative materials to make colours and products, rather than hand weaving techniques. Because there are few trees in the area, Prae Pun has to search for substitute materials for natural dyes. Some members, such as Raweewan and Walai, pointed out that they cannot cut a lot of bark from a tree at any one time because it will die. Furthermore, most members mentioned that Prae Pun conducted experimentation to search for varieties of colours and materials for natural dyes. Kamolwan and Pitsamai explained that each village group had to demonstrate natural dyeing with a locally available material in their villages in last year. Through this experimentation, members found several trees in their villages that can give beautiful colours. However, committee members still asked members to redo the experiments in order to confirm results based on the particular situation of each village. In a sense, the process of redoing can be viewed as knowledge verification. Many members, such as Prarichat and Raweewan, mentioned that natural dyes depend on nature and that each village has a different environment. Raweewan pointed out that members in her village use makleo for making dark colours, whereas members in other villages use mud instead because their villages do not have makleo, and members at Suksomboon village use rusty water to make dark colours. 
Group study also leads to new product creation, such as rug making. As with new designs, new products also emerge from the integration of creativity, weaving experience, local direction and trial and error. Raweewan stated that the manager had an idea about making rugs with the float patterns used in big blanket making. However, the weaving technique for big blankets could not be applied to make rugs. Therefore, Raweewan and members in her village group had to search for a rug making procedure through group work and observation. She explained that they discovered the techniques of rug making when one member, produced the same rug as the manager's original idea, after using different equipment than the other members. This process of rug making technique discovery can be interpreted as a community of practice (CoP), according to Wenger and Snyder (2000), because Prae Pun members work together in an informal group of similar interests in order to learn and share from each other. This also fits with the findings of Wenger, McDermott and Snyder (2002) that CoPs encourage innovation.

Therefore, both individual and group experimentation at Prae Pun involves knowledge creation, with a focus on procedures rather than skills, and knowledge verification. The experimentation is associated with knowledge verification by utilising the knowledge acquired and reviewing the results.

\subsubsection{Working}

Regarding the unique product offering of the community, members are free to design their cloth, especially the strip and plaid cloth that is designed uniquely for the monthly presentation of new ideas. Members need creative ideas and inspiration while they are preparing the warp threads. For example, Suwannee and Pitsamai mentioned that they imagine designs and try to use many thread colours when they are setting up the looms. Lada and Pitsamai explained that other members ask them for ideas when they are designing. The narratives show that new design creation requires creative ideas and sharing among members while they are working. This research shares the findings of Hansen, Nohria and Tiernery (1999) that a customised product offering strategy must involve knowledge sharing among organisational members in order to create unique products for customers. Moreover, the findings broadly accord with Nonaka's (1994) view of the relationship between knowledge sharing and knowledge creation. 


\subsubsection{Serendipitous discovery}

The narratives indicate that members sometimes discover new knowledge- both new techniques and new products—by accident. Malai stated that she realised that coconut husk could give a beautiful colour when dyeing with rusty water when she saw the colour on her knife as she was removing the coconut husk. After that members tested and experimented how to combine the coconut husk with rusty water for dyeing, and discovered how to prepare rusty water by using steel instead of having to get it from deep wells. Members also found new products by serendipity. Lada and Napaporn mentioned that they discovered saifon cloth when they used leftover mudmee weft thread without paying attention to the design. They delivered that cloth to the office and customers liked it, so now, they prepare mudmee weft for saifon weaving.

Serendipitous knowledge discovery is associated with declarative knowledge because it is concerned with facts. Members need to search for exact procedures. However, that is not difficult for them because they have previous knowledge that they can relate to new findings. In a sense, members use both new findings-declarative knowledge-and previous experience to create procedural knowledge and verify the knowledge discovered. In short, knowledge creation through serendipitous discovery comprises learning by observation.

In summation, the processes of new knowledge creation entail declarative and procedural knowledge creation and knowledge verification through experimentation, working and serendipity based on previous knowledge. Significantly, for this study, new knowledge creation relies on the skills and creativity of Prae Pun members. In this way, the findings broadly support Sternberg and Lubart (1991) research, which acknowledges that creativity is normally derived from people who have a certain level of knowledge in the area. The new knowledge creation of Prae Pun is associated with the CoP, which involves learning and sharing among members. According to Nonaka (1994), knowledge sharing in order to amplify individual concepts leads to organisational knowledge creation, while utilising organisational knowledge creates further knowledge. In line with Swan, Scarbrough and Hislop (1999), and Zack (2002), the processes of Prae Pun can also be viewed as knowledge utilisation because members have to utilise their previous knowledge, experience, observations and skills in order to create new knowledge. 


\subsection{Conclusion for knowledge processes of Prae Pun}

The analyses of the data in this research suggest that, even though Prae Pun does not have explicit knowledge management processes, members are aware of the need for knowledge acquisition and competence building. Several activities conducted for these purposes are associated with organisational knowledge creation and indigenous knowledge systems.

\subsubsection{Knowledge processes}

The analyses of data in this research suggest that the activities of Prae Pun can be viewed as processes of knowledge creation, capture, sharing, transfer, verification, utilisation and codification. Figure 7.2 shows all the knowledge processes and activities of Prae Pun.

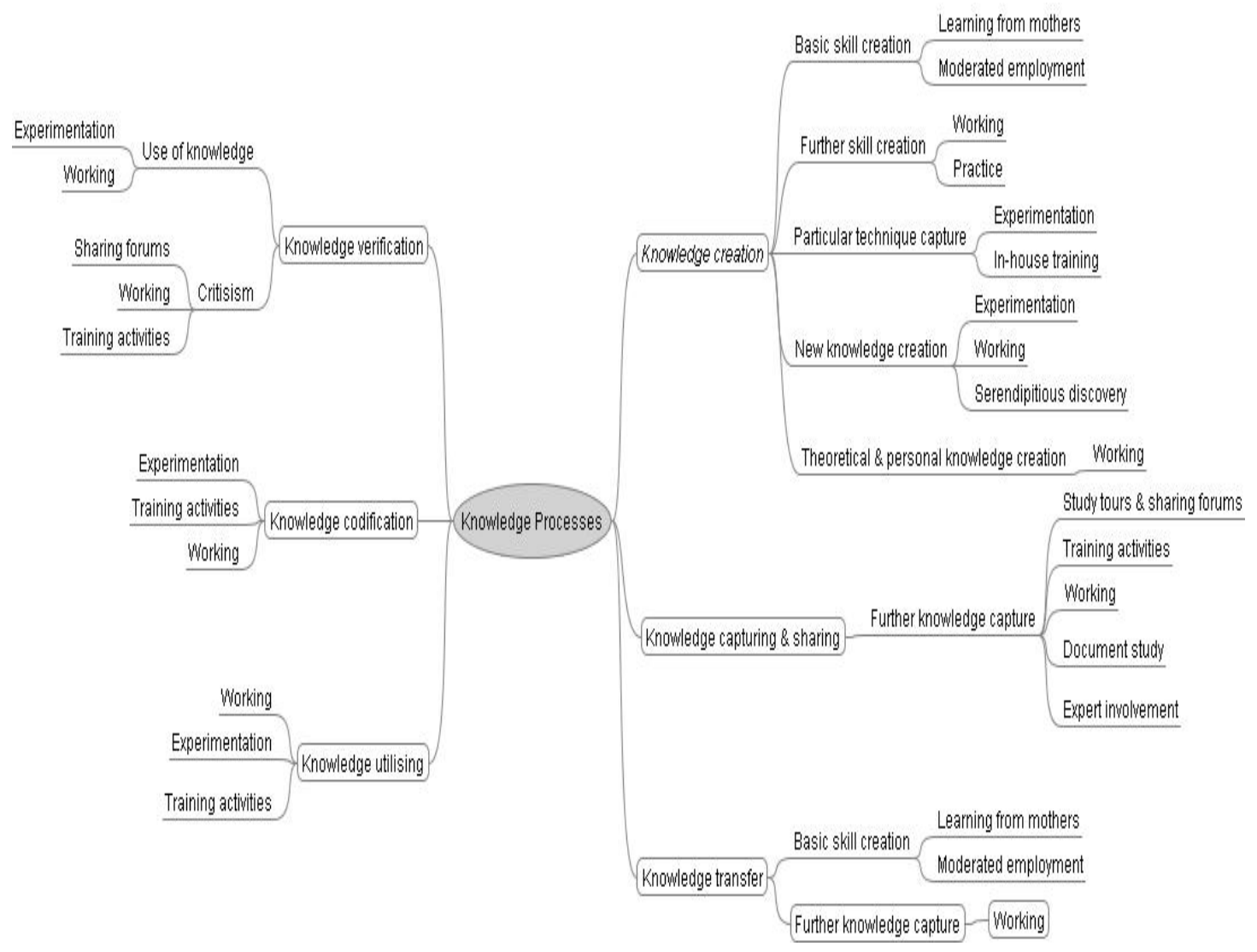

Figure 7.2 All knowledge processes of Prae Pun

\subsubsection{Knowledge creation}

This study suggests that knowledge creation of Prae Pun focuses on generating procedural knowledge-skills, further techniques or methods, and new knowledge 
through learning by doing and observation. However, these processes also imply theoretical and personal knowledge creation. First, the skill development focuses on three layers: generic skill creation, contextual skill creation and further skill creation. The generic skill creation is concerned with learning from mothers because hand weaving is women's responsibility. Contextual skill creation occurs through moderated employment programmes, and further skill creation comes from working and practice. Second, further technique capture is associated with particular procedure creation. Members search for procedures that match their situations through experimentation. Third, the new knowledge creation focuses on new products and designs, and particular technique creation through experimentation, working and serendipitous discovery. Fourth, the collaborative environment of Prae Pun activities also fosters theoretical and personal knowledge creation through organisational routines and socialisation, enabling members to make sense of shared values, and recognise the abilities and skills of other members. They can observe and learn the working styles and practices of other members which can influence their own work. These shared assumptions and beliefs can be viewed as theoretical knowledge, according to concept of knowledge types introduced by Badaracco (1991) and Choo (1998). The concept of social identity theory introduced by Tajfel (1978), and Ashforth and Mael (1989) suggests that members of the same society are able to identify the abilities and interests of individuals. This recognition can be viewed as personal knowledge, according to the findings of Skyrme (1999), and Van Den Bosch and Van Wijk (2001). Therefore, Prae Pun activities involve all types of knowledge creation in terms of members' organisational routines and learning by doing and observation.

\subsubsection{Knowledge capture and sharing}

The processes of further knowledge capture involve both internal and external knowledge. Initially, external knowledge capture at Prae Pun came about through training activities, study tours and sharing forums, document study and local expert involvement. Recently, the community has focused more on internal knowledge capture of customer, supporters and villagers' knowledge through organisational routines, communication, and socialisation. Knowledge sharing mainly occurs through informal collaboration and working environments 


\subsubsection{Knowledge transfer}

The knowledge processes of Prae Pun also involve transferring knowledge from senior members to new members and the younger generations who will become new members. The knowledge transfer occurs through teaching by mothers and other women villagers, moderated employment programmes, formal training activities and working. The narratives in this research suggest that the knowledge transfer of Prae Pun focuses on tacit knowledge, as discussed by Carrillo, Robinson, Anumba and Bouchlaghem (2006). This is because the knowledge transfer of Prae Pun is based on a human-centred approach rather than through tools.

\subsubsection{Knowledge verification}

Prae Pun members verify and test new knowledge to ensure its appropriateness before applying it to work processes. They occur through the methods of criticism, utilising and reviewing knowledge. The criticism occurs through sharing forums, committee meetings, work and training activities which facilitate discussion that allows members to critique the knowledge captured. Verification by utilising knowledge and reviewing the results occurs through experimentation and day-to-day working.

\subsubsection{Knowledge codification}

Significantly for this study, even though members are not familiar with documentation, formal activities are recorded in terms of performances and results. These activities include committee meetings, training, group experimentation and sales activities. The reports contain knowledge of hand weaving techniques, production processes and marketing details. As such, they can be viewed as knowledge codification, even though several researchers including Holsapple and Joshi (1999) and Al-Hawamdeh (2003) consider the process of encoding knowledge only in relation to explicit forms focusing on use of ICTs. Prae Pun's documents are created on the word-processor, but then printed off and stored in the files, rather than on the computer. These documents also represent organisational memories. Furthermore, Prae Pun's knowledge is stored in tacit form in several places, including members's minds, organisational routines, work processes, organisational structures and cultures. The narratives of this study reveal that members can easily retrieve knowledge to perform activities without opening their notebooks and documents (see section 7.3.1.4). These findings fit with the concept discussed by Walsh and Ungson 
(1991) that organisational memory can be stored in several locations including individuals, culture, transformations, structures and ecology, and can be retrieved with several mechanisms, both formal and informal.

\subsubsection{Knowledge utilisation}

Prae Pun can be viewed as a knowledge intensive organisation because its production requires the skills and experience of members to make cloth rather than solely their labour. The narratives in this research reveal that the community's members are active learners who pay close attention to acquiring knowledge and applying it to their work processes. However, only usable knowledge—skills, procedures, practical experience, work guidelines (shared values)—can be utilised for work processes. Facts and observations are processed through knowledge verification and usable knowledge creation, such as experimentation and on the job training. The concept of knowledge utilisation of Prae Pun derives from demand-driven approaches and operational aspects rather than access to computer-based applications as suggested by Alavi and Leidner (2001), and Dalkir (2005). Members are encouraged to apply knowledge to work processes rather than access knowledge repositories.

\subsubsection{Organisational knowledge creation}

Furthermore, the knowledge processes of Prae Pun can be interpreted as correlating with the organisational knowledge creation model introduced by Nonaka (1994) in order to generate knowledge assets, as shown in Figure 7.3.

In accordance with Nonaka (1994), the knowledge processes of the Prae Pun KM model can be regarded as knowledge enlargement, amplification, crystallisation, justification and networking knowledge. First, the processes of basic and further knowledge capture aim to enlarge individual knowledge, by providing members with opportunities to increase their range of experiences and capture all types of information from a range of sources. Second, Prae Pun's basic and further knowledge capture can be associated with Nonaka's amplification process, because they are concerned with sharing and transferring knowledge among members in order to create basic understanding and perception of how to work for Prae Pun, as a means of creating further organisational knowledge. 


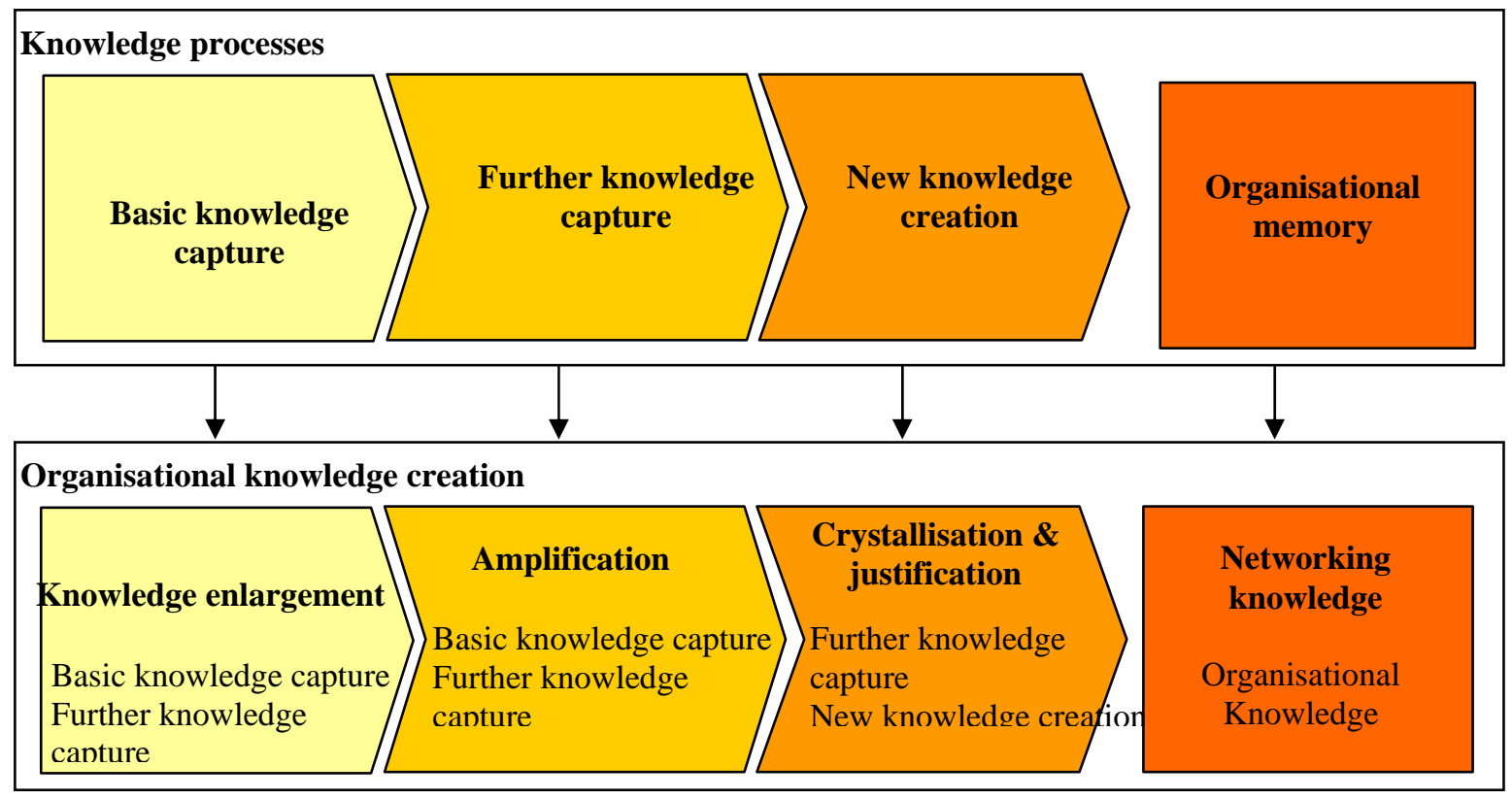

Figure 7.3 Matching of the processes of the KM model of Prae Pun with knowledge creation model of Nonaka (1994)

Third, the processes of further knowledge capture and new knowledge creation can be linked with crystallisation because Prae Pun's members utilise knowledge captured from several activities to create particular techniques, products, and designs which are concrete forms of knowledge. Fourth, knowledge capture of Prae Pun is associated with knowledge verification which can also be viewed as justification because members evaluate the appropriateness or truthfulness of knowledge within particular contexts. Lastly, the organisational memories of Prae Pun can be regarded as networking knowledge for organisational knowledge creation, because the organisational memories are available for members to access and utilise for further knowledge development.

In conclusion, the knowledge processes of Prae Pun enlarge members' knowledge and competence building in order to create organisational knowledge. The organisational knowledge is verified its appropriateness and stored as organisational memories in the forms of documents and tacit forms which are made available through mechanisms, such as moderated employment programmes, training activities and work processes to retrieve the knowledge. 


\subsubsection{Indigenous knowledge systems}

The data in this research suggest that knowledge processes of Prae Pun can be viewed as indigenous knowledge systems. Prae Pun runs its business relying on indigenous craft knowledge, such as traditional Isan cloth designs, hand weaving and natural dyes. Even though the $\mathrm{CE}$ has acquired business and group management knowledge through working, all Prae Pun knowledge processes have the same characteristics as indigenous systems, including collective and dynamic generation of knowledge, emerging through direct experience, its holistic nature, and oral transmission.

First, knowledge of hand weaving and natural dyes of Prae Pun is a combination of traditional Isan craft knowledge and Prae Pun's production strategies and customer preferences. The findings of this research reveal the characteristics of indigenous knowledge, which include collective, dynamic and accumulative generation over the years, and continued development over time based on the natural environment and particular social phenomena (Obomsawin, 2000; Hansen and VanFleet, 2003). Prae Pun's cloth is made by using traditional patterns and the creative ideas of members. Natural dye knowledge is generated by using traditional knowledge obtained from senior villagers, NGOs and other hand-weaving groups, and from members' experimentation with local materials Meanwhile, business and group management knowledge is derived from the combination of experience of Prae Pun accumulated over 10 years. For instance, Prae Pun regulations and sets of practice are developed by its own committee members and office staff members.

Second, Prae Pun members' knowledge is derived from direct experienceexperimentation, working and observation of their daily activities. The findings of this research fit with the concept of indigenous knowledge discussed by others, such as Waldram (1986), Korma (1995), Obomsawin, (2000) and Storey (2005) because members capture knowledge by observation in natural environment through firsthand experience.

Third, Prae Pun's knowledge comes from the integration of traditional crafts, members' skills and creativity, and Prae Pun's particular situations. This accords with Obomsawin (2000) who pointed out that indigenous knowledge is holistic in nature and combines individual components, such as experience, know-how, creativity and 
perceptions, social context and ecosystems. For instance, rug making is generated at Huafai group because this village has woven blankets using the necessary float patterns for a long time. Meanwhile, the knowledge of natural dyes is good example of the combination of ecosystems. Each village makes natural dyes with different materials that are available locally. The narratives of this study also show that Prae Pun's knowledge is holistic in nature as it comprises the characteristics of all its members and communities, which entail the situations and cultures of both the villages and Prae Pun.

Finally, Prae Pun's knowledge is transferred from generation to generation through the indigenous knowledge methods discussed by many authors, such as Korma (1995), Sefa Dei, Hall and Rosenberg (2000), and Storey (2005), including oral transmission and direct experience. As discussed in section 7.2, members learn from observing their mothers, the teaching of senior villagers, working together with senior members, the moderated employment and committee support programmes, and training activities.

Altogether, the knowledge processes of Prae Pun are representative of indigenous systems that focus on collective and dynamic generation of knowledge, through direct experience, and the holistic nature and traditional methods for knowledge transfer.

\subsection{Chapter summary}

The findings presented in the chapter, and the narratives of the members of Prae Pun focus on the capability development for members in order to make cloth and manage the CE. The more the development of members, the more the development of the enterprise. Prae Pun emphasises three areas of members' capability development: basic skill development, competence building and new knowledge creation. These areas involve knowledge creation, knowledge capture and sharing, knowledge transferring, knowledge verification, knowledge codification, and knowledge utilisation, all of which contribute to organisational knowledge creation. The capability development of Prae Pun correlates with the five processes of the organisational knowledge creation model introduced by Nonaka (1994)—individual knowledge enlargement, knowledge amplifying, crystallisation, knowledge justification and knowledge networking. Moreover, the knowledge management of 
Prae Pun can be viewed as indigenous knowledge systems. On this basis, the data in this chapter will be combined with the characteristics and situations of Prae Pun discussed in chapter 6 in order to generate a model of the community's current KM practice in the next chapter. 


\section{Chapter 8}

\section{A Knowledge Management Model for Prae Pun}

This chapter develops a knowledge management (KM) model based on the findings in the previous chapters, explored through the narratives of Prae Pun members and the researcher's observations, in order to describe the current KM practice of Prae Pun. The KM model is used to answer the research question 1: "how does the community enterprise currently manage its knowledge?” Because Prae Pun does not have an explicit KM system, some KM issues which are possible based on its characteristics and situations will not be considered, such as knowledge codification and knowledge transfer with tools. Then, the model will be refined to strengthen the existing KM practice in the light of the second research question-'Can theoretical and modern KM concepts be applied in an appropriate manner to strengthen traditional KM practice of the community enterprise?',

\subsection{Knowledge management model for Prae Pun}

To achieve the objectives of this research, the following KM model has been created in order to describe the current KM practice of Prae Pun. The findings of this research including the characteristics and knowledge processes of Prae Pun are summarised in tables 8.1 and 8.2 .

Table 8.1 The characteristics of Prae Pun

\begin{tabular}{|l|l|}
\hline \multicolumn{1}{|c|}{ Areas } & \multicolumn{1}{c|}{ Characteristics } \\
\hline Operation and management systems & - Committee systems \& open discussion \\
& - Self-employment \\
& - Job sharing \\
& - Home-based production \\
& - Unique product offering \& cloth standards \\
\hline Organisational cultures & - Community spirit \\
& - Sense of ownership \\
& - Informal and participation orientation \\
\hline Resources & - Local materials and human resources \\
& - Funding from members, NGOs and a \\
& customers \\
& - Social capital \\
\hline
\end{tabular}


As shown in table 8.1, Prae Pun operates and manages its enterprise by allowing members to actively participate in in a variety of roles, such as weavers, committee members and office staff members. Meanwhile, the production system provides opportunities for members to work in informal and collaborative environments which encourage learning and sharing. The organisational cultures of Prae Pun are concerned with socialising, flexibility and commitment. In terms of resources, Prae Pun relies on available materials in communities, members as human resources, traditional craft knowledge, funding from members' shares and social capital-the relations of members and other contacts, such as support organisations and customers.

Even though the members of Prae Pun do not consider their activities in terms of knowledge processes, those activities can be categorised as the processes of basic skill development, competence building and new knowledge creation as shown in table 8.2

Table 8.2 The current knowledge management practice of Prae Pun

\begin{tabular}{|l|l|}
\hline \multicolumn{1}{|c|}{ Activities/methods } & \multicolumn{1}{c|}{ Knowledge processes } \\
\hline - Learning from mothers & Basic skill development \\
- Moderated employment programmes & \\
- Committee support programme & \\
\hline - Study tours and sharing forums & Competence building \\
- Training activities & \\
- Practice and working & \\
- Document study & \\
- Experimentation & \\
- In-house training activities & \\
- Local expert involvement & New knowledge creation \\
\hline - Experimentation & \\
- Serendipity discovery & \\
- Community of practice & \\
- Working &
\end{tabular}

The basic skill development can be seen in the traditional method and knowledge transfer from skilful members to new members and non-skilful members. The competence building activities are those that focus on educational activities and collaboration that influences members learning from each other. Meanwhile, the new knowledge creation occurs when members are engaged in production because they to have design unique products with trial and error methods, and serendipitous discovery. 
Regarding the findings of this research, a KM model of current Prae Pun practice can be created comprising six components-knowledge resources, the operation and management systems, organisational cultures, resources, organisational knowledge assets, and knowledge processes which are located at the middle of the model. Furthermore, the KM is addressed within the community environment. Figure 8.1 shows all the components of the current KM model and their functions which will be discussed in detail in the following sections.

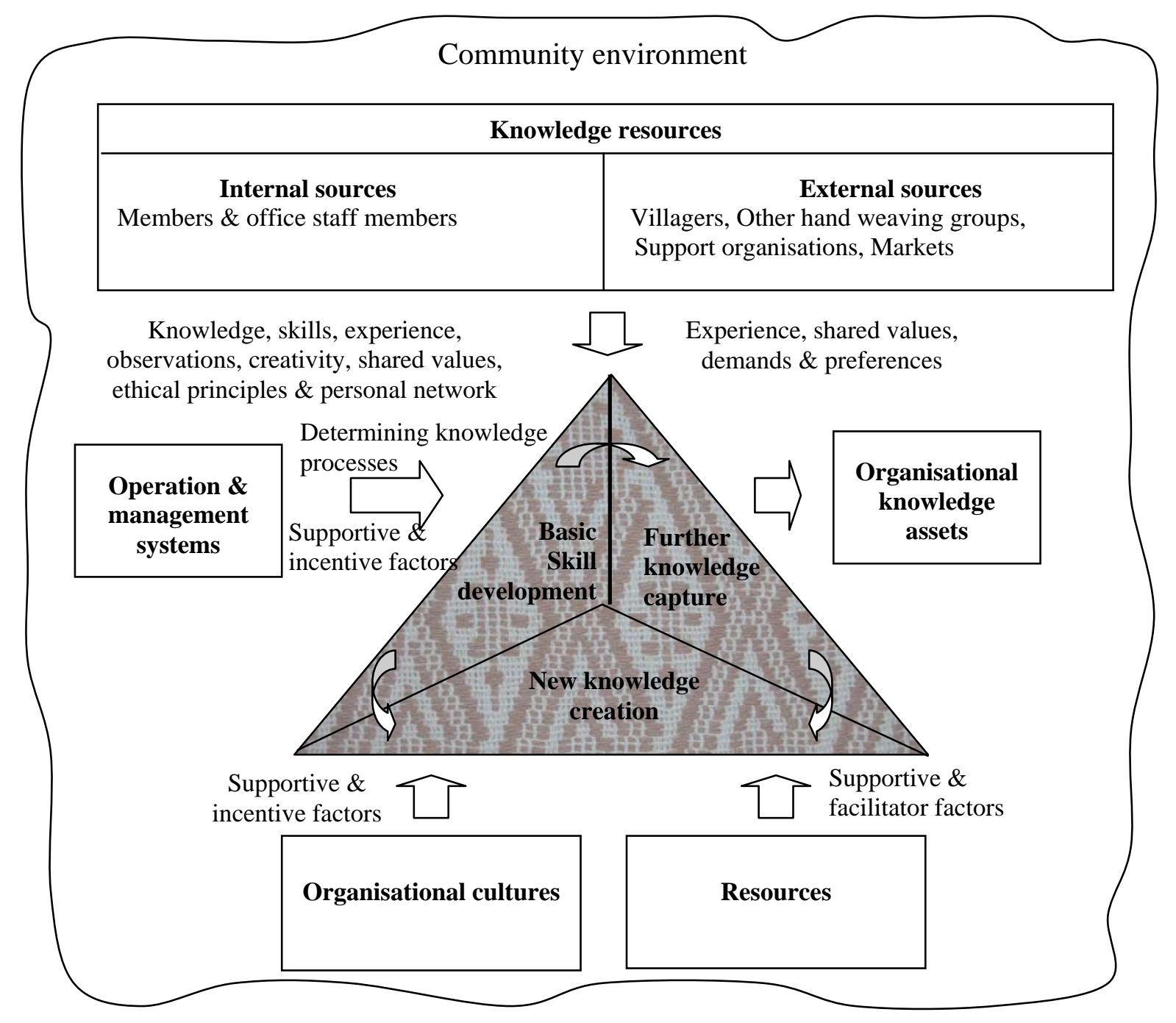

Figure 8.1 A current knowledge management model of Prae Pun

\subsubsection{Knowledge}

Regarding the products and production system, Prae Pun relies on traditional craft knowledge captured from members-internal sources, other villagers and other hand weaving groups. Prae Pun also obtains knowledge from other associated parties- 
support organisations and markets—which are external sources. Figure 8.2 shows the knowledge resources obtained from each group.

Knowledge sources

\begin{tabular}{l|l|l|l|l|}
\hline \multicolumn{1}{|c|}{ Internal sources } & \multicolumn{3}{|c|}{ External sources } & Markets \\
\hline \multicolumn{1}{|c|}{ Members } & Villagers & $\begin{array}{c}\text { Other hand } \\
\text { weaving groups }\end{array}$ & $\begin{array}{c}\text { Support } \\
\text { organisations }\end{array}$ & Demands \& \\
preferences \\
Exills, \\
Creativity, \\
Shared values, \\
Ethical principles, \\
Personal network
\end{tabular}

Figure 8.2 Knowledge resources for the KM model of Prae Pun

\subsubsection{Knowledge from members}

Because of Prae Pun's self-employment system, the knowledge of its members is a crucial resource. The narratives of this research reveal that all members have at least basic skills at hand weaving when they become members. Even office staff members have to know about hand woven cloth in order to work at the office. After becoming members, they obtain and create several forms of knowledge-skills, experience and observations, creativity, shared values and personal knowledge. Furthermore, members also utilise ethical principles in the performance of activities.

\section{Skills}

The findings identified by this research fit with the findings of others, such as Sanchez (1997), Nahapiet and Ghoshal (1998), and Skyrme (1999), that skills comprise the practical understanding and abilities that enable people to perform tasks. Prae Pun's members possess both generic and contextual skills in order to perform 
activities. Regarding village culture, most members gain generic skills at hand weaving from their mothers or woman villagers before becoming members. In terms of contextual skills, new members obtain these from long-term members through moderated employment programmes in order to prepare them to work. Both generic and contextual skills enable members to acquire further skills and knowledge through working and activities conducted by Prae Pun. Thus, most long-term members have higher and more advanced skills than new members.

\section{Experience and observations}

Prae Pun also depends on members’ experience for both production and office work. For production, members use their experience to make cloth suitable for particular situations, such as the natural environments of each village and customer preferences. In terms of business operation and management, Prae Pun relies more heavily on the practical experience of office staff members rather than the regulations and decisions of committee members. Furthermore, members obtain knowledge from the observation of aspects such as product samples and management strategies, which are utilised for their practice, experimentation and day-to-day working in order to capture further knowledge, and create new products and new designs. Such knowledge can be viewed as declarative knowledge, according to the concept of knowledge creation discussed by several researchers including Ryle (1949), Garud (1997), Brown and Dugid (1998), and Lam (2000), because the observations are derived from the perception and judgment of members and utilised for procedural knowledge creation.

\section{Creativity}

The creativity of Prae Pun members is a vital resource for new knowledge creation. New products and new designs are created based on the innovation and imagination of skilful craftspeople. This study supports the findings of Woodman, Sawyer and Griffin (1993), and Sternberg and Lubart (1996) which associate creativity with new inventions, products and levels of skills. The can be viewed as declarative knowledge according to the knowledge types discussed by Nahapiet and Ghoshal (1998) because creativity is knowledge at the state of thinking that requires practical engagement in order to search for suitable procedures and methods. 


\section{Shared values}

The shared values of Prae Pun-community spirit, sense of ownership and participation orientation-influence members' behaviour and performance. The narratives of this research reveal that members are willing to participate in activities, share knowledge and learn new things. Shared values can be viewed as theoretical knowledge, according to the knowledge types discussed by Badaracco (1991) and Choo (1998), because they guide and form the behaviour and actions of organisational members.

\section{Ethical principles}

It is evident from the literature review that virtue is a part of extraordinary scope of knowledge or wisdom (Aristotle, /2002). Ethical principles are also inherent within Prae Pun's operation and management, and guide members, especially committee members, in their actions. For instance, ethical principles are one of the criteria for selecting committee members and also motivate the cloth inspection team to work honestly. Given this, ethical principles can also be viewed as theoretical knowledge, according to Badaracco (1991) and Choo (1998), because they govern members' conduct.

\section{Personal network}

It is found in this study that members realise the abilities of their fellow members and can refer to those members when they need help. This constitutes personal knowledge, according to the knowledge types discussed by Skyrme (1999), and Van Den Bosch and Van Wijk (2001) because this network is concerned with knowingwho- the abilities and interests of other members in the organisation. Such findings broadly support the study of Dooley et al. (2002), which recognises that individuals in organisations seek to learn the abilities of others, thereby influencing the creation of knowledge directory.

Given this, the KM model shows that knowledge from members can be classified as procedural knowledge, declarative knowledge, theoretical knowledge and personal knowledge. This knowledge is primarily tacit and indigenous knowledge located in members' minds and in their hands. The findings of this research are similar to a KM model proposed by KMI (2005a), which reveals that the knowledge within people 
organisations and the public sector in Thailand mainly takes the form of members' experience and local wisdom, culture and beliefs.

\subsubsection{Knowledge from Villagers}

Because Prae Pun applies a home-based production system relying on traditional craft knowledge, members also look to villagers who are not members of the community for alternative sources of knowledge. The narratives in this study reveal that such villagers willingly share their experience in hand weaving and natural dyes with Prae Pun members. Members also collect examples of cloth designs that have been woven for several generations within the villages. The knowledge captured from villagers constitutes declarative knowledge according to the characteristics presented by Ryles (1949), and Nahapiet and Ghoshal (1998) because it is concerned with observations and facts that members gain without direct experience. Members need further studylearning by doing - in order to acquire procedures for remaking cloth and designs. This research also accords with the proposed KM model of KMI (2005a) that the KM for people organisations and the public sector in Thailand generally comprises knowledge from local experts.

\subsubsection{Knowledge from other hand weaving groups}

Like the villagers, other hand weaving groups, especially groups in the same region, share their experience in hand weaving, natural dyeing and group management with Prae Pun. Compared with the experience from villagers, experience from other hand weaving groups is contemporary and practical. It has been developed for modern products and the other hand weaving groups are similar to Prae Pun in terms of the type of enterprises and their characteristics. However, as with Prae Pun members' observations of villagers, their observations of other hand weaving groups can be viewed as declarative knowledge, which members have to search for particular procedures.

\subsubsection{Knowledge from support organisations}

The narratives in this research reveal that the main support organisations for Prae Pun are NGOs and FTOs. They also share their experience, information and shared values to support Prae Pun activities. The sharing from NGOs comes mainly in the form of documents about traditional Isan cloth and natural dyes, and their experience at 
management. Furthermore, Meanwhile, FTOs mainly contribute their experience in cloth design and marketing. As with the experience from other hand weaving groups, knowledge captured from NGOs and FTOs can be classified as declarative knowledge because it comprises facts and members' thinking and perception. This study is similarly the KMI Thailand (2005a) that the KM for people organisations and the public sector includes knowledge from external sources including NGO workers and scholars, and is mainly explicit knowledge in the form of documents. However, Prae Pun focuses on contact with NGOs and FTOs. As a result, the knowledge captured from support organisations of Prae Pun is more tacit than the knowledge captured from scholars and government.

\subsubsection{Knowledge from markets}

Prae Pun also utilises customer knowledge and trends which are derived from markets for its production and business operation. The knowledge captured from markets can be viewed as customer capital according to the intellectual capital concept discussed by Stewart (1997) because it is concerned with demands and customer preferences, and relationships with them. As with knowledge from other hand weaving groups and the support organisations, the knowledge captured from markets can be viewed as declarative knowledge because it is concerned with facts and knowledge at the state of thinking and perception without members' practical engagement.

In short, the KM model of Prae Pun is associated with knowledge of indigenous craft and CE management from five sources: members, other villagers, other hand weaving groups, support organisations and markets. This research supports the findings of others, such as Choo (1998), and Holsapples and Joshi (1999), that organisational knowledge creation requires information from several sources including organisational members, customers, suppliers, consultants and researchers. Meanwhile, knowledge from external sources tends to be declarative knowledge, which is the basic resource of practical engagement in order to acquire procedural knowledge. Knowledge from members, on other hand, is concerned with skills, experience and observations, creativity, shared values, ethical principles, and personal networks which enable members to perform Prae Pun activities. 


\subsubsection{Operation and management systems}

The analyses of the narratives in this study suggest that the operation and management systems of Prae Pun determine its knowledge processes by focussing on members' ability development and new knowledge creation. Furthermore, the systems also provide support and incentives for its KM. Figure 8.3 illustrates the operation and management systems of Prae Pun that are associated with its KM.

\begin{tabular}{|l|}
\hline \multicolumn{1}{c|}{$\begin{array}{c}\text { Operation \& } \\
\text { management systems }\end{array}$} \\
\hline - Self-employment \\
systems \\
- Production systems \\
\hline - Committee systems \& \\
open discussion \\
- Self-employment \\
system \\
- Job sharing \\
- Production systems \\
\hline
\end{tabular}

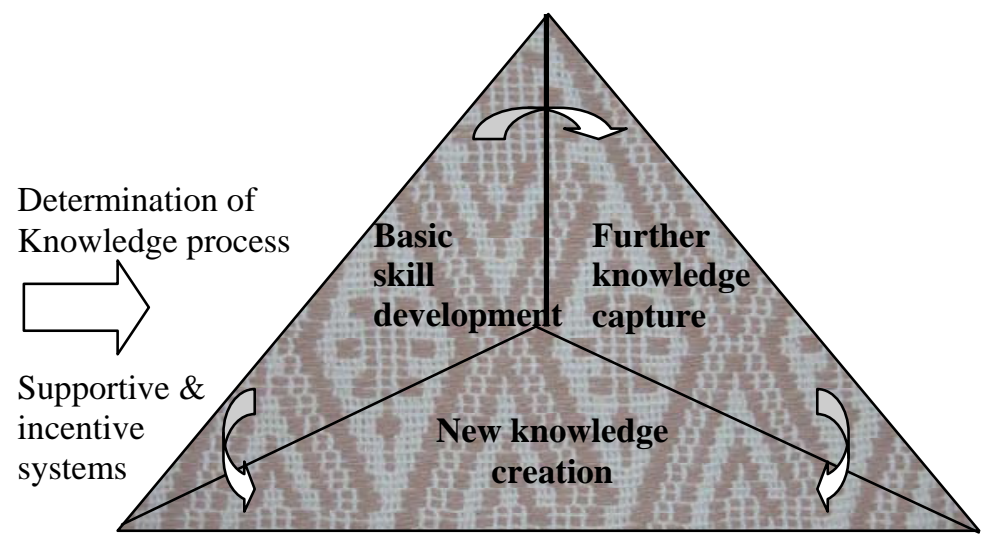

Figure 8.3 The roles of operation and management systems for the KM model of Prae Pun

\subsubsection{Determining knowledge processes}

The findings in this research reveal that operation and management systems fit with the KM model introduced by Collison and Parcell (2004) that acknowledges that business activities decide the knowledge processes of an organisation. The selfemployment and production systems determine the knowledge processes which focus on members' competence building and new knowledge creation. First, because of the self-employment system, Prae Pun has to pay close attention to members' competence development in both production, and operation and management. The findings in the previous chapter show that Prae Pun stresses the importance of both skill development and enlarging members' knowledge. Second, in terms of production, the unique product offerings and cloth standards influence the knowledge processes which focus on further knowledge capture and new knowledge creation in order to acquire techniques to make varieties of cloth with high quality. Therefore, the operation and 
management systems contribute to Prae Pun members’ competence building—skill development and further knowledge capture-and new knowledge creation.

\subsubsection{Supportive and incentive systems}

The operation and management systems of Prae Pun provide supportive and incentive systems for its KM because they are associated with a collaborative environment, learning processes, and reward and incentive systems.

\section{Collaborative environment}

The committee, job sharing and production, systems can be viewed as collaboration which involves both physical and management aspects. First, the committee systems and open discussion, job sharing, and home-based production can be interpreted in terms of the management aspect of a collaborative environment because those systems are concerned with decentralisation which encourages members to share their responses and opinions. Second, the home-based production system also represents the physical aspect of a collaborative environment because members' homes are open spaces, where the weavers often visit each other and work together in groups. The office also has an open layout, where all the staff can interact. Both the production and the office environments broadly reflect the findings of Handzic and Agahari (2004) that decentralisation and open office layout can enhance knowledge sharing.

\section{Learning processes}

The operation and management systems of Prae Pun are associated with problem identification, knowledge acquisition, testing and reflection, knowledge storing, and knowledge distribution which Handy (1995) and Lopez, Peon and Ordas (2004) classify as learning processses. First, the committee and open discussion systems address problem finding and solving, as well as critiquing and revewing knowledge by encouraging members to discuss and share opinions with each other. The committee support programme can be viewed as knowledge acquisition and distribution, while the committee work also focuses on generating documentation which can be seen in terms of knowledge storing. Second, the job sharing system involves interaction among office staff members which can be also viewed as knowledge acquisition. Third, the production system involves members in the process of knowledge acquisition in order to search for new techniques and products. 
In the mean time, production also involves testing and reflection because members always verify knowledge captured by utilising and reviewing the results.

\section{Reward and incentive systems}

The committee systems can be also viewed as intrinsic reward and incentive systems according to the concept suggested by Bartol and Srivastava (2002), because the honour of being committee members is an intrinsic value,concerned with recognition and appreciation by members, which can facilitate competence building (Amabile, 1993). The systems encourage committee members to develop their capabilities in order to be accepted by members. In terms of reputation, committee members are recognised as being skilful and dedicated to the community, willing to share their knowledge and support less skilful members. In a sense, the very systems themselves encourage committee members to share their knowledge. In a wider view, this research broadly supports the findings of Bartol and Srivastava (2002) that reveal that reward and incentive systems significantly reduce the reluctance of organisational members to disseminate their knowledge.

Overall, the operation and management systems can be seen to play a role in determining Prae Pun's knowledge processes, and providing incentives for its KM. The self-employment and production systems, comprising collaboration, unique product offering and cloth standards, govern its knowledge processes focusing on competence development and new knowledge creation. Meanwhile, the committee systems are associated with collaborative environments and learning processes, and represent intrinsic reward and incentive systems that enhance the community’s KM.

\subsubsection{Organisational cultures}

The data in this research suggest that an organisation's culture has a significant influence on its KM. It is evident from the literature review that $\mathrm{KM}$ is best fostered by cultures that value learning (Despres and Chauvel, 2000) and sharing (Smith and McKeen, 2005). The organisational cultures of Prae Pun are KM-driven, and can be viewed as reward and incentive systems. Figure 8.4 shows all the Prae Pun organisational cultures which contribute to the existing KM model. 


\subsubsection{Knowledge management-driven culture}

The organisational cultures of Prae Pun are associated with both sharing and learning. First, the community clearly values both knowledge sharing and cognitive sharing. All aspects of Prae Pun's culture encourage members to exchange knowledge with each other. In a wider view, the findings of this research broadly support the findings of others, such as Moffett, McAdam and Parkinson (2002), about the impact of collaborative organisational culture on KM. Meanwhile, the community spirit and the sense of ownership can be seen as cognitive sharing, because such values motivate members to understand Prae Pun strategies and adapt to the environment.

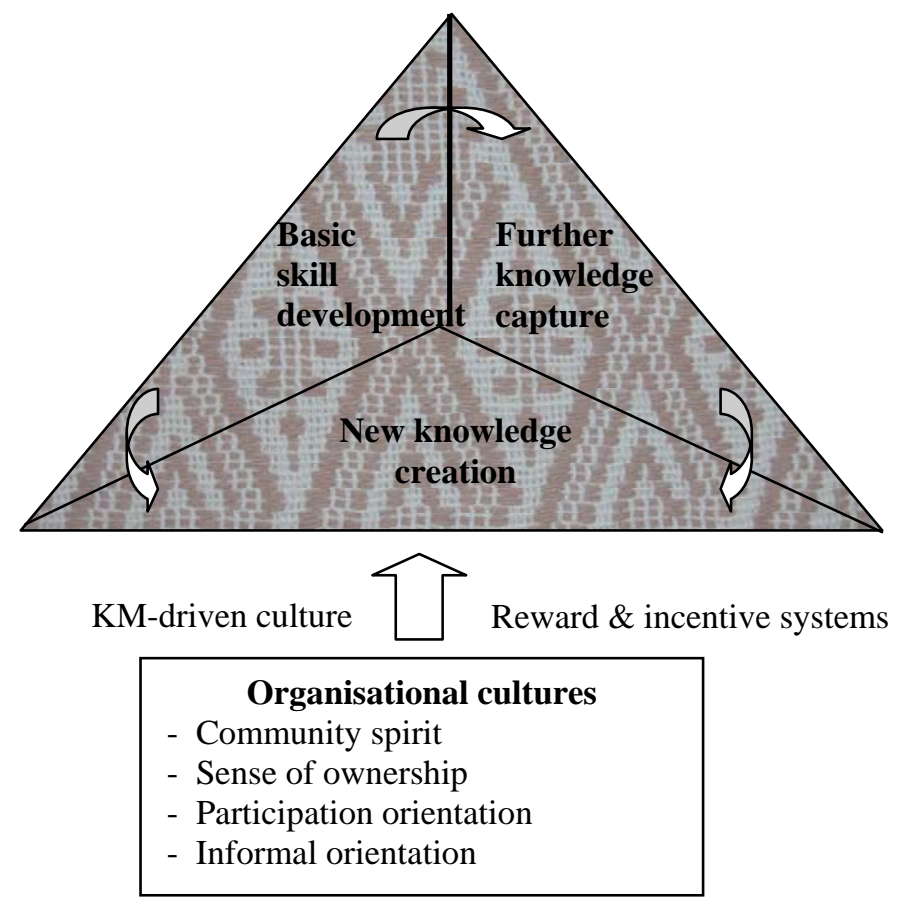

Figure 8.4 Organisational cultures as supportive incentive systems for the KM model of Prae Pun

Second, the community spirit and the sense of ownership also constitute learning culture. It is evident from the literature review that learning culture is concerned with shared assumptions (Schein, 1992) and organisational wisdom (Despres and Chauvel, 2000). The community spirit fosters learning because a high standard of weaving ability is one of the distinguishing characteristics of Prae Pun community members. Meanwhile, the sense of ownership influences learning as a means towards organisational goal achievement, as the members have high commitment to develop themselves for the good of Prae Pun community as a whole. In this way, these 
findings accord with Capello and Faggian's (2005), observation that a sense of belonging is crucial in order to encourage learning amongst members of an organisation.

\subsubsection{Reward and incentive systems}

Prae Pun's community spirit and members' sense of ownership can be also viewed in terms of the reward and incentive concept discussed by Amabeli (1993), and Bartol and Srivastava (2002). Such rewards are intrinsic with the community placing great importance on the recognition, appreciation, satisfaction and interest of members in improving their competencies. Meanwhile, the sense of ownership enhances members' high commitment and motivation to learn and create new knowledge, and share this knowledge throughout the community. This research supports the findings of others, such as Bartol and Srivastava (2002), and Gopalakrishnan and Santoro (2004), that a sense of ownership and commitment can serve as motivation for individuals to share knowledge about new technologies and knowledge capture. Moreover,, the findings confirm Amabeli’s (1993) view, that intrinsic values and feeling of individuals can encourage creativity-generating new inventions, products and abilities.

Altogether, the organisational cultures of Prae Pun provide support for its KM, by incorporating both sharing and learning cultures, which can be viewed as intrinsic reward and incentive systems.

\subsubsection{Resources}

Resources such as funding, social capital and appropriate technologies-telephones and desktop computer-are associated with Prae Pun's KM as part of the reward and incentive systems and tools. Figure 8.5 illustrates all the resources contributing to the community's KM and their roles. 


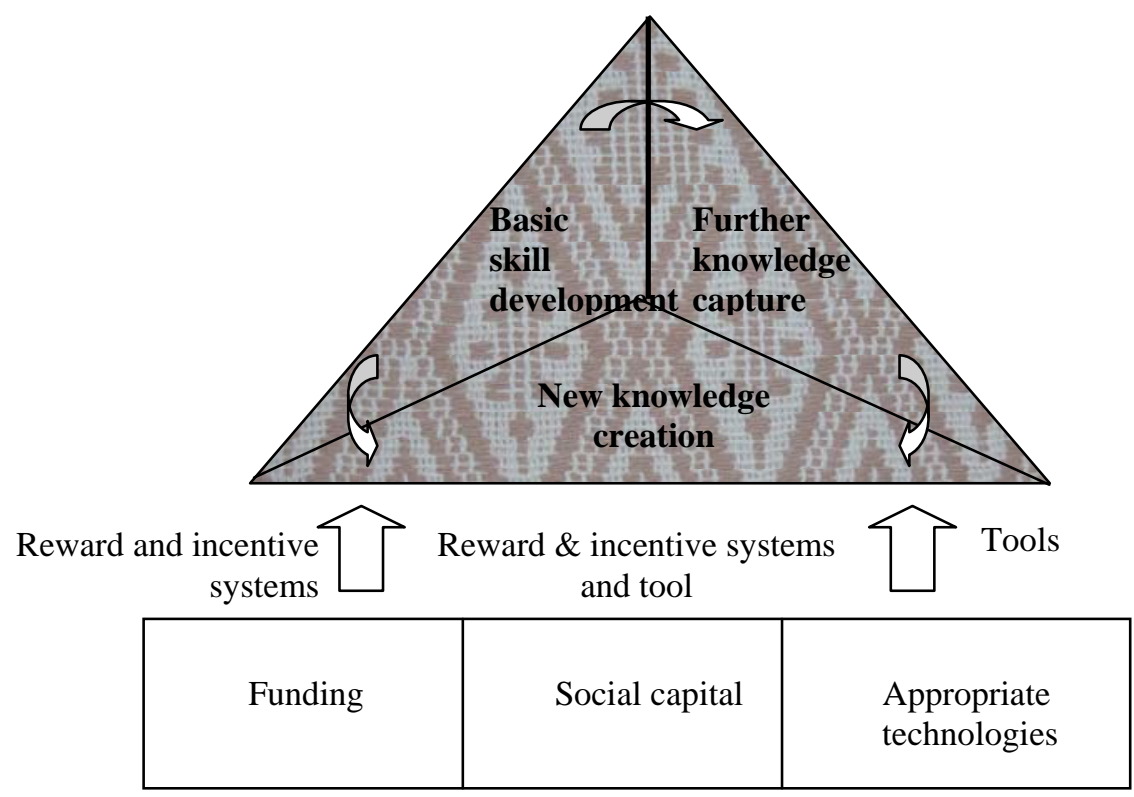

Figure 8.5 Resources for the KM model of Prae Pun

\subsubsection{Funding}

Funding is the one aspect associated with the reward and incentive systems of Prae Pun's KM model. Members earn income from the sale of their cloth, and Prae Pun provides money for members' welfare, such as heath care, children's education funds, loans, and senior members' allowances. These benefits can be viewed as 'extrinsics in service of intrinsics', introduced by Amabile (1993, p.194). Those benefits provide tangible incentives in order to facilitate members in working and learning. The narratives in this research reveal that most of Prae Pun's members have been able to improve their standard of living and children's education thanks to the income and member loans from the community.

\subsubsection{Social capital}

Social capital can comprise both intrinsic reward and incentive systems, and, as such, the results of this case study endorse the findings of Nahapiet and Ghoshal (1998), Capello and Faggian (2005), and Hoffman, Hoelscher and Sherif (2005), that social capital can facilitate knowledge management. First, the social capital exists in the form of social norms, such as obligation and expectation, according to the reward and incentive systems discussed by Amabile (1993), and Bartol and Srivastava (2002). This is because the social norms influence members to appreciate Prae Pun's values of learning and sharing. Meanwhile, the high commitment and responsibilities in 
learning and sharing are associated with the appreciation, satisfaction and interest of members in participatory knowledge processes. Second, network ties provide information and information channels to facilitate human capital creation, as noted by Coleman (1988), and Nahapiet and Ghoshal (1998). The social relations among the community's seven village groups, customers and support organisation staff form channels which serve as a tool for knowledge sharing and transfer. In short, Prae Pun's social capital in the form of community norms, obligations and expectations constitute intrinsic reward and incentive systems which encourage learning and sharing among members. Meanwhile, the social networks can also be viewed as a tool for knowledge processes.

\subsubsection{Appropriate technologies}

The narratives in this research reveal that Prae Pun does not rely on ICTs for its KM, even though many researchers, such as Sena and Shani (1999), Reid (2003), and AbuRashed, Bertaux and Okunoye (2005), highlight the role ICTs can play in facilitating the interaction of organisational members. Currently, the only technologies involved in the KM of Prae Pun are telephones and a computer with common software for both documentation and communication facilitation, such as an email service, at the office level. This is because the villagers are more familiar with face-to-face communication and the community is limited in terms of both funding and computer literacy skills. This finding accords with Koanantakool (2003), who notes that rural families tend to have trouble meeting daily expenses let alone the cost of computers and internet services, and that public internet services are difficult to establish at the rural community level.

Nevertheless, Prae Pun does employ a desktop computer with common programmes for documentation, which can be viewed as a knowledge codification process. This is because the documentation produced can be viewed as a part of content management, which according to Wyllie (1998), and Moffett, McAdam and Parkinson (2004) is a crucial technique to facilitate knowledge codification. Furthermore, as shown by Ungson (1991) and Nilakanta, Miller and Zhu (2006), this can be also viewed as organisational memories because Prae Pun's documents record what Prae Pun does and knows, in the forms of committee meeting minutes and reports from the formal training activities. Even though members of the weaving collective are less interested 
in capturing the knowledge contained in Prae Pun's documents, the community as a whole, at the organisational level, pays attention to the storing of its collective memories through technology.

Telephones play an important role in enhancing knowledge sharing and transfer between office staff members and community members, and amongst office staff members, advisory committee members, support organisations and customers. Prae Pun, especially at the office level, uses both land-line and mobile phones for business operation and management. Meanwhile, communication within village groups mainly relies on face-to-face communication. Even thought most members have mobile phones, they tend to use them solely for private matters.

Therefore, although it is a rural, home-based organisation, Prae Pun employs technologies such as telephones and a desktop computer with common software for documentation and communication as tools to facilitate its KM through knowledge sharing, transfer, codification and capture of organisational memories. In a wider view, this research reinforces the findings of Sparrow (2001) that the KM of SMEs tends to rely on off-the-shelf software and basic internet services rather than more advanced systems.

Altogether, Prae Pun has adequate resources to support its KM. Even though some researchers (Wong and Aspinwall, 2004) point out that small enterprises tend to have limited funding for extrinsic reward systems, Prae Pun has some funds set aside for reward and incentive systems in the form of 'extrinsics in service of intrinsics'. Prae Pun also utilises its social capital in the form of supportive and incentive systems as a tool to facilitate its KM, as well as appropriate technologies.

\subsubsection{Knowledge processes}

Based on its self-employment and production systems, Prae Pun's KM encompasses the processes of basic skill development, further knowledge capture or competence building, and new knowledge creation, in order to develop members' competence and the community's business ventures. Figure 8.6 shows all the processes and relationships of the KM model for Prae Pun. 


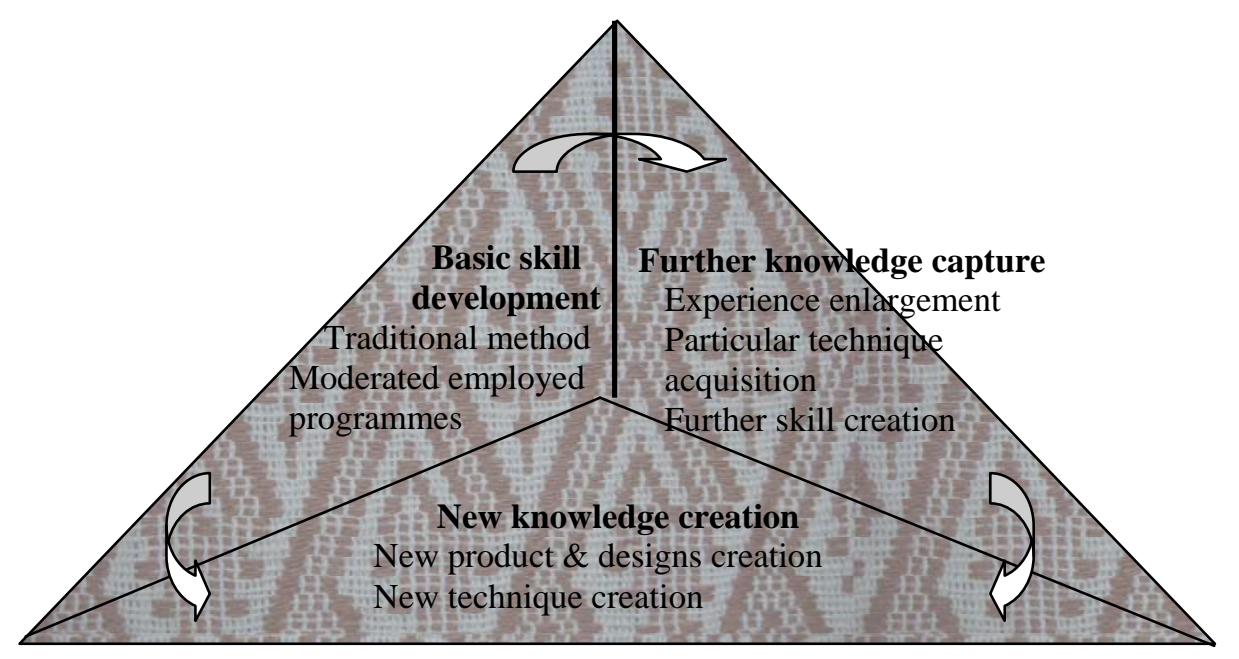

Figure 8.6 The knowledge processes of the KM model of Prae Pun

\subsubsection{Basic skill development}

As summarised in table 8.2, basic skill development within Prae Pun derives from traditional methods-members learning from their mothers or other female villagers-and from moderated employment programmes, like new member orientation, internship programmes and a committee support programme. On this basis, basic skill development is associated with acquiring both generic and contextual skills. The generic skills are learnt from mothers or senior villagers, while the contextual skills are developed by means of the moderated employment programmes. Both skill development programmes are concerned with learning by doing and knowledge transfer from generation to generation or from skilful to novice weaver in order to prepare new members to work, capture further knowledge and contribute to organisational knowledge.

\subsubsection{Further knowledge capture}

Further knowledge capture describes members’ ongoing competence building in order to develop Prae Pun capabilities in terms of both production and business management. The further knowledge capture comprises the processes of experience enlargement, further technique capture and further skill development. First, the experience enlargement occurs through several activities: study tours to other hand weaving communities and sharing forums, training activities, working in collaborative and informal environments, and document study. The collaborative and informal dayto-day working environments allow members to interact and capture knowledge from 
both fellow members and others including villagers, support organisations and customers. However, the knowledge captured from those activities is declarative, because members simply observe, rather than having the opportunity to practise. Knowledge gained through working in collaborative environment can also be defined as procedural, because members capture it through learning by doing. However, members constantly need to verify and adapt their skills and particular procedures to match with their situation. Furthermore, the collaborative and informal working environments of Prae Pun also facilitate theoretical and personal knowledge capture through socialisation and collaboration.

Second, another aspect of competence building is further technique capture, which utilises basic knowledge together with ongoing experience and observations to create particular procedures. This process occurs in Prae Pun through experimentation, onthe-job training and expert involvement. The narratives of this research reveal that skilful members can practise or conduct experimentation themselves after study tours or training activities. Non-skilful members, on the other hand, require on-the-job training under the supervision of skilful members in order to capture further techniques. This shows that capturing further techniques relies on previous skills and knowledge. Moreover, these findings broadly support studies by Cohen and Levinthal (1990), and Gupta and Govindarajan (2000b) which suggest that the level of prior related knowledge and familiarity of incoming knowledge enhance new knowledge recognition and absorption. Furthermore, Prae Pun also captures further techniques by regularly inviting local experts to participate its activities. Lastly, another form of further knowledge capture is the ongoing skill development, which occurs through members' working and practising in their free time. The narratives in this research suggest that the number of hours spent in such ways contribute to members' acquisition of further skills for both cloth production and office work.

In short, Prae Pun increases the competence of members by enlarging members' experience, skills and particular knowledge. The further knowledge capture occurs through several endeavours, which can be grouped as educational activities, and working in collaborative and informal environments. These activities allow members to capture all types of knowledge from both internal and external sources. The internal knowledge capture comprises procedural and tacit knowledge acquisition through 
members' learning by doing and socialisation. Meanwhile, external knowledge capture entails declarative knowledge capture through observation and documentation. The narratives in this research suggest that the level of basic knowledge possessed by members has an impact on further knowledge capture. As shown in the literature, skilful organisation members are able to capture knowledge with an independent process faster than lesser skilled members.

\subsubsection{New knowledge creation}

New knowledge creation consists of generating new designs, products and techniques through experimentation, working with unique product offerings and serendipitous discovery. In Prae Pun experimentation entails a process of learning by doing, a trial and an error method combined with the previous knowledge, creativity and local direction of senior members. The experimentation occurs for both individuals and groups through formal group study and informal CoPs (as described in Chapter 7.4.1). Working, on the other hand, creates knowledge through the sharing of ideas and skills in order to create new designs or unique products. Both the experimentation and working can be regarded as procedural knowledge creation and knowledge verification, as members utilise knowledge and review the results. Significantly for this research, members are constantly deriving new knowledge through serendipitous discovery while working or engaged in daily activities. The knowledge acquired through serendipitous discovery can be classified as declarative knowledge because it is a fact for which members need to develop exact procedures and skills. Considerably, the new knowledge creation is concerned with creativity, a certain levels of previous knowledge and skills, and local guidance. As a result, basic knowledge capture and further knowledge acquisition are vital prerequisite processes for new knowledge creation of Prae Pun.

In conclusion, the knowledge processes of Prae Pun focus on developing members' capabilities in order to equip members to become self-employees who work for Prae Pun, and create new knowledge in order to achieve the production strategies-unique product offerings and strict system of cloth standards. Even though Prae Pun does not have an explicit KM system, the model presented in this chapter demonstrates that its activities can be considered in terms of the knowledge processes, classified as basic skill development, further knowledge capture and new knowledge creation. These 
activities include traditional methods — teaching by mothers and female villagers, and telling by villagers_educational activities, engaging in tasks and socialisation. Such forms of learning by doing and observation involve all types of knowledge acquisition because Prae Pun's collaborative and informal working environments enhance interaction and socialisation among the community's related parties.

Indeed, the three knowledge processes of Prae Pun can be categorised into knowledge creation, capture and sharing, transferring, verification, utilisation and codification, all of which focus on traditional and informal methods. Knowledge capture and creation derives from learning by doing, direct observation and oral transmission. For knowledge transfer, it occurs through traditional methods-learning from mothers and villagers, and the sharing of villagers-moderated employment programmes and collaborative work, which focus on human-centred rather than tool-centred approaches. In terms of knowledge verification, the KM model involves applying and testing the knowledge and reviewing the results. Meanwhile, knowledge utilisation tends to be limited to operational aspects, such as word-processing, and demanddriven approaches, with members retrieving information only as necessary.

Prae Pun pays little attention to knowledge codification and utilisation of tools to facilitate its knowledge processes. Only knowledge acquired through formal activities (such as committee meetings, and training and experimentation sessions) is documented in a word processing format and kept in the office. Meanwhile, knowledge acquired through individual members' experimentation and serendipitous discovery is not recorded. Nevertheless, some members do record knowledge captured from study tours and training activities in their notebooks but they pay less attention to utilising and applying it. Furthermore, Prae Pun has two books containing its organisational memories. They outline the lessons learned over 10 years at Prae Pun but do not record any organisational knowledge, especially valuable tacit knowledge and specialised techniques, in detail. There are many possible reasons, such as limited understanding of of knowledge acquisition techniques and the importance of knowledge protection for business competition. Nevertheless, Prae Pun has a wealth of organisational memories stored in tacit forms within its members' minds, organisational routines, products and cultures. 
Moreover, the knowledge processes of Prae Pun seem to correlate with the organisational knowledge creation model introduced by Nonaka (1994). and can be viewed as knowledge enlargement, amplification, crystallisation, justification and networking. First, the knowledge enlargement can be seen from the processes of basic skill development and further knowledge capture, because both processes provide opportunities for members to expand their varieties of experience and capture all types of knowledge. Second, basic skill development and further knowledge capture also involve the amplification process because they prepare members to create particular techniques, products, and designs for Prae Pun. Third, the crystallisation and justification processes can be seen as evidence of further knowledge capture and new knowledge creation because they are creating new techniques, products, and designs which are concrete forms of knowledge, and also verifying the appropriateness of knowledge captured and created. Lastly, networking knowledge can be seen in the community's written documents and organisational memories.

\subsubsection{Organisational knowledge assets}

The last component of the KM model of Prae Pun is organisational knowledge assets. They are concerned with the intellectual resources resulting from the knowledge processes, which Hall (1992) classified as assets and competences In this way the Prae Pun knowledge processes aim to develop members' competence in order to create new knowledge and organisational knowledge.

\subsubsection{Assets}

The assets derived from the KM model comprise products and designs, particular techniques, sets of practices, and reputations, which are not registered as intellectual property, and are located in products and activities rather than formal documents or records. Members of Prae Pun believe that the designs and techniques created on the basis of Isan culture should belong to Isan society. In other words, they have a sense that things belong to one community by drawing upon and utilising by the community itself. The findings on knowledge assets broadly support the study of others, such as Obomsawin (2000), that indigenous knowledge is not registered but it is belong to a community because it is derived from community members, utilised, and transferred from generation to generation. Those new designs are recognised as Prae Pun's products because they are unique to Prae Pun and laden with Prae Pun contexts. For 
instance, even though products and designs may employ traditional patterns, they are made with specifc Prae Pun creativity, customer and product knowledge. Both members and long-term customers can recognise such cloth as Prae Pun products. Moreover, members can identify individual products, even pieces of products, in terms of the construction techniques of individual makers —-weavers and/or village groups-, because they recognise the abilities of each other and have basic skills at hand weaving all of Prae Pun products.

However, currently, such indigenous knowledge is threatened by both loss and exploitation through cultural extermination (Obomsawin, 2000). The narratives of this research suggest that traditional craft knowledge may have competitive advantages for an organisation. The members are aware of the importance of preserving their knowledge, especially the particular techniques that make their unique products. Nevertheless, the assets, such as products and designs, particular techniques and set of practices, are also located in documents, such as committee meeting reports and activity reports which serve as evidence to identify individual weavers. In terms of reputation, the narratives of this research show that Prae Pun is recognised as skilful at both hand weaving with natural dyes and community enterprise management. This research supports the findings of KMI (2005a) that the knowledge assets of people organisations and the public sector tend to be in the records of knowledge discovered and lessons learned from their activities.

\subsubsection{Competencies}

The knowledge assets in the form of competences encompass the skills and abilities of members, social capital and shared values which are embedded in members and their work processes. They are elusive and unique to each organisation because they are connected with the specific processes and cultures which facilitate organisational activities (Hall, 1992). Similarly, the competencies of Prae Pun are unique and valuable only within Prae Pun's context, such as the particular skills and abilities of each member and the community's specific production strategy of cloth standards and marketing plans. Second, although Hall (1992) defines the relations and networks associated with contracts and business relationships as assets, Prae Pun does not have any formal contracts with other parties. However, the informal social relations and networks among Prae Pun and associated parties can be seen as forms of social capital 
which foster specific information provision for Prae Pun. Third, as with know-how and social capital, shared values can be seen also as valuable competencies which facilitate Prae Pun activities.

Altogether, knowledge assets derived from the knowledge processes of the KM model can be seen in the community's assets_-products, designs, particular techniques and work practice —and in its competencies—skills and abilities of members, social capital and shared values. The knowledge assets of Prae Pun are not registered as intellectual properties but nevertheless they belong to the community because they are unique and valuable within Prae Pun contexts and exist as organisational memories which can be accessed and utilised for members' activities.

As depicted in Figure 8.1, the KM model for Prae Pun attempts to illustrate the relationship between all the knowledge management processes of the community (the internal and external knowledge resources, operations and managements systems, organisational cultures, actual resources, and organisational knowledge assets) and their contribution to members' basic skill development, further knowledge capture and new knowledge creation.

\subsection{Strengthening the KM model}

Because Prae Pun does not have an explicit KM system, the community places little emphasis on best using its resources or considering how situations can be maximised to facilitate KM. To achieve the objective of this research, the KM model outlined in 8.1 will now be refined by integrating appropriate modern $\mathrm{KM}$ concepts in order to strengthen the current KM practice. The data in this research suggest that Prae Pun has some potential to address modern KM concepts based on its existing KM with modern KM concepts possibly strengthening several components: in particular, the community's knowledge resources, operation and management systems, resources, knowledge processes, and knowledge assets.

\subsubsection{Knowledge resources}

Currently, Prae Pun focuses on utilising external knowledge mainly from its local contacts and relationships which are limited to NGOs and FTOs. In fact, Prae Pun could also seek knowledge from other organisations, especially from academic and 
government agencies that support CEs. This is because CEs are recognised as an effective solution for community development, especially in terms of promoting economic development and gender equality in developing countries (HalvorsonQuevedo, 1991; Kitahara, 2000). As a result, Prae Pun members could also expand their understanding by capturing knowledge from organisations which would provide particularly useful information for its businesses. It is evident from the literature review that people organisations in Thailand already access knowledge resources in the form of documents from scholars and government officers (KMI, 2005a). However, the knowledge captured from those sources tends to be limited to declarative knowledge, which, within Prae Pun, could be combined with practice in order to enhance the community's procedural knowledge creation and knowledge verification.

\subsubsection{Operation and management systems}

The current operation and management systems of Prae Pun have been developed without attention to KM, therefore Prae Pun does not have a distinct strategy for its KM. However, the findings in this research suggest that Prae Pun has the potential to develop its operation and management systems to further support KM, by incorporating learning processes and reward and incentive systems. First, even though the operation and management systems of Prae Pun can already be regarded as learning processes, some processes are given less attention. The effectiveness of members' learning processes, especially in relation to the storing and distribution of Knowledge, could be improved by developing more awareness of the processes of knowledge codification and use of tools. Issues relating to resources and knowledge codification will be discussed more fully in the following sections.

Second, the findings of this research show that Prae Pun currently does not provide any extrinsic reward and incentive systems for members who create or discover new knowledge. Those members have to spend their time learning and searching while experiencing economic hardship. Even though several researchers, such as Gupta and Govindarajan (2000b), Bartol and Srivastava (2002), and Hislop (2005) argue that extrinsic reward and incentive systems often reduce the motivation and willingness of organisational members, organisations can balance between reward systems and motivation by applying the 'extrinsics in service of intrinsics' system recommended 
by Amabile (1993). Despite the fact that Prae Pun already has such a system in place, in the form of welfare for members, it does not specifically serve to motivate new knowledge creation. Therefore, Prae Pun should consider providing financial rewards and incentives to acknowledge those members who devote their energies to creative work, and therefore may have less time to produce cloth for sale. . This issue also relates to resources which will be discussed in section 8.2.2.

\subsubsection{Resources}

This research has revealed that Prae Pun, members consider that they lack the necessary funding, ICTs, and human resources to operate the technologies. However, Prae Pun has the potential to manage both its finances and existing technologies to strengthen its KM. First, even though Prae Pun currently has insufficent funding for extra resources, such as hiring a professional office staff member to manage the business, it already has a benefit system to provide for members' welfare, loans, and business development. Moreover, the narratives of this case study indicate that Prae Pun members have plans to open a second showroom. This shows that Prae Pun has the capacity to manage its funding to further develop its business, and, by association, its KM. This suggests that, Prae Pun should be able to allocate some funding for an extrinsic reward and incentive system, in terms of extra payment for members with the motivation and abilities to create new knowledge. Second, Prae Pun already has the appropriate technologies for strengthening its KM. Those technologies include telephones and a desktop computer with common software for knowledge sharing and organisational memory.

\section{Telephone}

Even though currently the main method of communication at Prae Pun is face-to-face, telephones could be used more in order to enhance knowledge sharing and transfer between members, villages, office staff members, advisory committee members and support organisations. This is because most members have mobile phones and the office staff members already use both land line and mobile phones for some business operations. As shown by the literature review, telephones enable more informal communication, which can enhance knowledge transfer (Alavi and Leidner, 2001; Moffett, McAdam and Parkinson, 2004). However, as discussed in section 8.1.4.3, 
telephone bills might prove an economic burden for villagers. Therefore, telephone use by members at the village level should remain optional

\section{Computer and software}

Because a desktop computer, common programmes and some human resources are already available for Prae Pun, they should be exploited in order to strengthen its KM, especially in terms of knowledge codification and knowledge transfer. It is evident from the literature review that internet services are becoming widespread in developing countries (Litan, 2005). However, the use of Prae Pun's computer is likely to remain restricted to the office level because members at the village level would have difficulty affording computers and access the internet services. Prae Pun should better utilise its existing computer and common software, by developing a filing system for documenting organisational memories. In terms of communication, Prae Pun could use the internet services more for email communication with advisory committee members and support organisations in order to increase the community's effectiveness in sharing and transferring knowledge.

Altogether, even though unlikely to be able to afford professional office staff, Prae Pun already has adequate levels of funding and the appropriate technologiescomputer and telephones-to strengthen its existing KM. Prae Pun has potential to mange its finances in order to introduce an extrinsic reward and incentive system and to enhance knowledge codification and knowledge transfer.

\subsubsection{Knowledge processes}

Even though they actually employ several knowledge processes, Prae Pun members currently pay little attention to how knowledge codification, utilisation and transfer could increase the effectiveness of its $\mathrm{KM}$, especially knowledge utilisation and transfer within the community's network of villages, customers and support organisations.

\section{Knowledge codification}

Members at the office and management level deal with Prae Pun's documentation and organisational memories on a day-to-day basis and have the potential to use the existing technologies. Nevertheless, the community's KM could be further 
strengthened by utilising further techniques and tools for knowledge capture and codification.

First, there are possibilities to exploit internet, web technology and common programmes such as word processing and spreadsheets for document management, because they are already available at Prae Pun together with the necessary human resources. It is evident from the literature review that internet technologies and technologies for document management can support content management (Moffett, McAdam and Parkinson, 2004) which can, in turn, enhance knowledge storage and distribution (Alsup and Strong, 2004). However, these members should be further trained in document management, to use the softward to its full capacity, because few currently have sufficient skills in documentation and technology. Furthermore, Prae Pun should consider providing facilitators or consultants for members at the village group level, who could take notes in order to facilitate knowledge capture and codification. As Wenger (2004) notes, a CoP can be enhanced by the input of coaches and consultants. The narratives of this research reveal that the younger generation, especially the children of members, have higher education, and could well help Prae Pun members develop the necessary skills in documentation.

Second, Prae Pun could conduct activities to capture the knowledge contained in members' notebooks or minds as organisational knowledge and memories. The data in this research show that Prae Pun has potential to capture their lessons learned as documents with support from advisory committee members and NOGs. Prae Pun already has produced two books about its experiences as a hand weaving CE. Therefore, Prae Pun could conduct a similar project for knowledge capturing and management.

\section{Knowledge utilisation and distribution}

Even though Prae Pun conducts activities, such as moderated employment programmes, and collaborative working to encourage knowledge transfer to new members and non-skilful members, those programmes remain focused within each village group rather than within the network of all seven villages. Other activities, such as cloth delivery, committee meetings and annual meetings, also provide opportunities for knowledge sharing between members from different village groups, 
but those activities are not specifically directed towards knowledge transfer and distribution throughout the network. Some possible techniques that Prae Pun could employ include developing an information package for the moderated employment programmes and Prae Pun's product and design collections. Even though computers are not available at village group level, the researcher observed there are CD players. As a result, the knowledge package and Prae Pun product and design collection could be produced in the form of audio and visual sets that would increase access to the knowledge discovered by members from the different village groups. Such knowledge packages have been shown to encourage organisational members in knowledge utilisation and creativity (Bhatt, 2001). In terms of knowledge transfer, even though a tool-centred approach is not as rich a transmission channel as face-to-face communication, it is an effective technique for explicit knowledge transfer (Carrillo, Robinson, Anumba and Bouchlaghem, 2006). Knowledge package and Prae Pun product and design collection can be a supplement method for knowledge transfer and distribution which is concerned with tool-centre approach.

\subsubsection{Knowledge assets}

Due to the fact that most of Prae Pun's knowledge assets exist solely as members' competencies, they can be easily lost. In order to preserve these obscure assets, explicit forms of the knowledge would help. As a result, documents, the knowledge packages, and Prae Pun product and design collections could transform the knowledge assets of Prae Pun into obvious values. In a sense, those tools would transfer knowledge assets in the form of competences into actual asset forms in order to be recognised as Prae Pun assets, even though they are not registered.

As shown above, a number of modern KM concepts can be integrated into the current KM practice of Prae Pun in order to strengthen its current practice which focuses on traditional methods or indigenous knowledge systems, such as direct experience (doing and observation in particular environment), oral transmission and learning from elder community members. Aspects of modern KM, particularly better communication and document recording, more effective use of tools and technologies, the introduction of incentives and ongoing networking, could enable Prae Pun to develop a KM project in order to enhance its effectiveness in the creation and utilisation of knowledge, especially indigenous knowledge, for its businesses. 


\subsection{Chapter summary}

A KM model has been created based on the findings in the previous chapters to describe the current KM practice of Prae Pun. The model reveals that, Prae Pun manages its knowledge within a community environment in order to create organisational knowledge assets for its business operation and development. Those knowledge assets are mainly in the forms of competences or obscure things which are not registered as intellectual assets. However, they are unique and valuable within Prae Pun contexts and exist as organisational memories. The organisational knowledge of Prae is managed through a series of activities which can be viewed as three main processes: basic skill development, further knowledge capture, and new knowledge creation. The basic knowledge acquisition is very vital processes for members' competence development and organisational knowledge creation. The organisational knowledge of Prae Pun is derived from both internal and external sources. Even though knowledge from members is usable and crucial for conducting activities, knowledge capture from others including villagers, other hand weaving groups, support organisations and markets are vital sources for usable knowledge creation. The knowledge processes of Prae Pun are determined by its operation and management systems which emphasise on self-employment system and knowledge intensive production system-knowledge and skill based and quality centre. Furthermore, the KM of Prae Pun is reinforced by its organisational culture which contributes to the KM model in terms of supportive and incentive systems, and its resources-funding, social capital and appropriate technologies - that are supportive and incentive systems and tools to facilitate the knowledge processes. Given that Prae Pun has the typical characteristics of other CEs, especially CEs in developing countries, this KM model answers research question one, by outlining "how the community enterprise currently manages its knowledge”.

Lastly, the KM model is refined by the integration of some modern KM concepts, such as knowledge storage and distribution over the network, tool utilising and extrinsic reward and incentive systems, based on its potential in order to strengthen its current practice. The refined KM model suggests that currently, 'theoretical and modern KM concepts could be applied in an appropriate manner to strengthen the traditional KM practice of the community enterprise'. 


\section{Chapter 9 \\ Conclusion and Implications}

This chapter will provide a summary overall of this research: objectives, research questions, research design, the findings of this research and limitations in order to help other researchers who are interested in KM for micro and community enterprises, especially the enterprises in developing countries, fast capturing characteristics of KM for those organisations. This chapter will also discuss the implications of how to utilise this KM model for practitioners-community enterprises and support organisations — and finally the limitations and implications for further research.

\subsection{Conclusion of the research}

9.1.1 Objectives and research questions

This research was conducted with the aim to explore the characteristics of a community enterprise (CE) including the operation and management systems, resources and organisational cultures, and activities related to knowledge processes in order to create a KM model. To date there has been no research focusing on KM for CEs, even though they are a crucial part of community development in developing countries (Welsch and Kuhns, 2005), and have many obstacles restricting their growth. Staff members have low level of education and business skills (TID, 2003), and they have limited resources (Halvorson-Quevedo, 1991). KM can be a vital factor to strengthen their growth. However, KM models for large and small and medium enterprises (SMEs) are not appropriate. This is because CEs are micro enterprises with specific characteristics, in terms of management systems, resources, business types and objectives. Thus, this research has sought to explore the characteristics and current KM practice of Prae Pun in order to create a KM model for a CE in response to the two research questions

1). How does the community enterprise currently manage its knowledge?

2). Can theoretical and modern KM concepts be applied in an appropriate manner to strengthen traditional KM practice of the community enterprise? 


\subsubsection{Research design}

This research employed a qualitative approach with narrative research to seek understanding of a community enterprise's characteristics: operation and management systems, culture, and current KM practice. The data were collected through narrative inquiry, participant observation and document analysis, and were analysed through the theme analysis techniques introduced by Boje (2001). Prae Pun-a hand weaving community enterprise in rural Thailand-was selected as a research site, because it has the typical characteristics of community enterprises. In order to increase the quality of this research, validity, credibility, reliability and ethical issues were taken into account. In terms of validity, the accuracy and correctness of accounts were addressed with data triangulation. As the data were collected in Isan dialect and translated into English, advice from native speakers was sought in order to increase descriptive validity. Generalisation and transferability were ensured by thick description of contextual information. This research was conducted under the criteria of Victoria University's Human Ethics Committee in order to ensure that this research did not harm respondents or violate their privacy.

\subsubsection{The findings of this research}

The analyses of the data in this research reveal that there is significant evidence to support the research questions. The answers were generalised from the situation at Prae Pun which served as a representative case study for CEs.

\subsubsection{Answer to research question one:}

How does the community enterprise currently manage its knowledge?

This question was answered through developing a KM model for Prae Pun based on the findings of this research, including the CE's particular characteristics (operation and management systems, organisational cultures, resources, and benefits) and its knowledge resources, operation and management systems, organisational culture, resources, knowledge processes, and knowledge assets.

Prae Pun's knowledge resources come from five sources: members, other villagers, other hand weaving groups, support organisations and markets. Most knowledge is concerned with tacit knowledge in the forms of skills and experience rather than 
explicit knowledge. The CE's operation and management systems determine the knowledge processes by focusing on competence building and new knowledge creation, and provide reward and incentive systems in the form of 'extrinsics in service of intrinsics'. The organisational cultures also provide supportive and incentive systems in the form of intrinsic rewards. Resources for the KM model include funding and social capital in the forms of both extrinsic and intrinsic systems, and appropriate technologies as tools to facilitate the knowledge processes. Social capital can also be viewed as a tool to facilitate the knowledge processes. The KM model outlined in this study encompasses three processes: basic knowledge capture, further knowledge capture and new knowledge creation which contribute to organisational knowledge creation in terms of the model introduced by Nonaka (1994). Knowledge assets comprise assets—products, designs, techniques, and operation and management systems-and competences-the skills and abilities of members, social capital and shared values. The knowledge assets of Prae Pun are not registered as intellectual property but are recorded in documents, such as committee meeting reports and formal activity reports, and in tacit forms: members' minds, products and organisational routines, which can be accessed and utilised for its activities.

\subsubsection{Answer to research question two:}

Can theoretical and modern KM concepts be applied in an appropriate manner to strengthen traditional KM practice of the community enterprise?

The data in this research suggest that modern concepts can be possibly intergrated into the traditional KM practice of Prae Pun, based on the CE's potential and situation in order to strengthen current practice. Those KM concepts could include:

- Seeking knowledge resources from further sources, such academic and government agencies. Even though the knowledge from those sources tend to emphasise declarative knowledge, this type of knowledge is vital for usable knowledge creation within the CE.

- Further developing use of technologies, such as document management systems, knowledge tool kits and telephones, to facilitate learning processes (storage 
and distribution) and knowledge processes, especially knowledge codification and knowledge transfer.

- Utilising facilitators or consultants from the local community and external support organisations, to enhance the community's CoP and knowledge capture and codification.

- Applying extrinsic reward systems for outstanding members who create new knowledge.

- Capturing lessons learned and producing documentation and knowledge package projects in order to preserve this knowledge.

\subsection{Implications of the research}

\subsubsection{Implications for Practice}

This KM model will benefit the KM development projects of both Prae Pun and other CEs, because it provides guidelines to identify the characteristics and situations of CEs in order to verify and adapt the roles of each component $l$ to match with current situations. There are some particular practical issues for both Prae Pun and other CEs to consider in the use of the KM model.

\subsubsection{Implications for Prae Pun}

In order to use this model productively, Prae Pun will need to assess its current resources, operation and management systems, and activities related to knowledge processes in order to specify contents that may change from time to time. Knowledge resources may depend on the particular contacts at the time, for instance, involving government agencies, and academic organisations as well as the current NGOs. This is because currently, CEs are widely accepted as a solution for community development in developing countries, especially in terms of economic and gender development (Halvorson-Quevedo, 1992; Kitahara, 2000). As a result, government, and academic institutions could further support CEs especially regarding development of business management skills.

Other resources may change according to specific situations and contexts. For instance, in the future Prae Pun may be able to gain more profits, which could be managed to enhance its KM. Over time, it may have to acquire more and higher levels of appropriate technologies, because ICTs are developing rapidly and becoming 
widely diffused in developing countries (Litan, 2005), and rural communities may gain more opportunities to utilise ICTs for their production and communication. In terms of human resources, currently, the younger generation has more opportunities in education and in exploring the outside world. If they gain skills in management and technologies, they will be able support Prae Pun as staff members and volunteers.

Current operation and management systems need to be evaluated for functions that may affect KM, especially those determining the knowledge processes. Prae Pun may need to develop some strategies, such as member development programmes and contracts with other parties, such as FTOs and customers, that might be advantageous for its KM. Therefore, Prae Pun may have some features that need to be developed in order to support its KM more explicitly.

\subsubsection{Implications for other CEs}

This research reveals that CEs can be classified into different sub-categories, such as supported versus independent, rural versus urban, religious or culture-based versus other, and those in developing versus developed countries. These sub-categories would all affect the application of the KM model, which would need to consider the specific context of each component based on the particular environment. Examples of some sub-categories of CEs are discussed as follow:

\section{Supported CEs and independent CEs}

Government supported CEs would have different knowledge resources and support systems from independent CEs. For instance, most knowledge resources of government-supported CEs may come from central agencies, which are more concerned with declarative knowledge. Thus, government-supported CEs should pay attention to utlising that knowledge to create procedural knowledge. Meanwhile, independent CEs may need to develop further knowledge acquisition through document study and study tours. In terms of support, government-supported CEs may have funding from government agencies which can be used for extrinsic reward and incentive systems, whereas independent enterprises may need to generate their own. 


\section{Urban CEs}

Urban CEs focus on manufacturing rather than traditional production (Wasuntiwongse, 1999) and community members come from a variety of places (Burke, 1970). Urban CEs may obtain less knowledge from members because their experiences and skills tend to be varied. Therefore, urban CEs may need to capture usable knowledge for their enterprises from other communities.

\section{CEs in other culture communities}

Buddhist beliefs strongly influence the organisational cultures and KM of Prae Pun as all its members are Buddhist. CEs in other cultural or religion-based contexts need to identify members' beliefs and values in order to utilise them as organisational, support and incentive systems.

\section{CEs in developed countries}

Unlike CEs in developing countries, CEs in developed nations tend to be more complex and concerned with regulations (Schreiner, 2001). Thus, their operation and management systems might be more formal. As a result, CEs in developed countries need to identify their management advantages in terms of KM. Moreover, CEs in developed countries are likely to have more opportunities to utilise ICTs to facilitate their KM, especially for communication among members.

\subsubsection{Limitations of this research and implications for further research}

This research focused on a single case study, Prae Pun, over a period of two months, May-July 2006, identifying the specific characteristics of a rural indigenous Thai CE supported by NGOs. Furthermore, because this research focused only current KM practices, other potentially interesting issues, such as intellectual properties and economic issues received less attention. In order to expand the knowledge of KM into areas such as micro enterprises and indigenous knowledge management system, four aspects are suggested for further study.

\section{1) Appropriateness of the KM model for CEs}

In order to serve as a guideline for other CEs, the KM model created in this research must be fully assessed in terms of its appropriateness for application in the wider CE 
environment, especially rural CEs, in order to examine the facilitators and barriers to success.

\section{2) Characterising KM model for particular CE types}

As discussed in section 9.2.1.2, CEs can be classified into a number of sub-categories according to specific characteristics and environments. Research should also be undertaken in these other forms of CEs, in order to determine the specific functions and roles of each component of the KM model.

\section{3) Indigenous knowledge management systems}

Many CEs draw upon traditional craft knowledge and indigenous knowledge systems. There is a need to further investigate each knowledge process (knowledge creation, capture, sharing, transfer, verification, utilisation and codification) in terms of indigenous knowledge and wisdom management systems, especially focusing on organisational context and intellectual asset management.

Research into these three areas would expand understanding in the areas of knowledge management, indigenous knowledge systems and community and micro enterprises.

\subsection{Conclusion}

In conclusion, this research has explored the characteristics of a rural community enterprise, its operation and management systems, resources, activities organisational culture, and current knowledge management practice. It also suggests possible ways to strengthen traditional indigenous KM practices by integrating modern KM concepts according to its situation. The KM model created in this research could serve as a guideline for other CEs to develop KM projects. Moreover, this study fills a gap in academic study into KM for micro enterprises and indigenous knowledge, and other disciplines including organisational study and social development, especially rural and gender development in developing countries. 


\section{Glossary}

\begin{tabular}{|c|c|}
\hline 4-Ariyasaj & Four noble truths \\
\hline 4-iddhipatihariya & Power of transformation \\
\hline $2-K a o$ & $\begin{array}{l}\text { Standard weaving technique with two heddle } \\
\text { shafts }\end{array}$ \\
\hline 3-kao & Weaving technique with three heddle shafts \\
\hline 4-kao & Weaving technique with four heddle shafts \\
\hline Aoy & Elder sister \\
\hline Arjan & $\begin{array}{l}\text { 'A master’ but Northeast villagers use as title } \\
\text { before names of NGO workers. }\end{array}$ \\
\hline Ban or moo ban & Village \\
\hline Bog & Isan almond \\
\hline Bug hyay & A design for float pattern weaving \\
\hline Bun Phawes & A ceremonial banner \\
\hline Dukkha & The natural truth of suffering \\
\hline Eye & Elder brother \\
\hline Huklwan & Indian almond \\
\hline Isan & Name of ethnic group in northeast of Thailand \\
\hline \multirow[t]{3}{*}{ Jug jig } & Jug jig is the name of float pattern that Prae \\
\hline & Pun’s members use for big blanket and rug \\
\hline & making. Jug jig means fussy. \\
\hline Kao & Heddle shaft \\
\hline Kit & Supplementary weft weaving cloth \\
\hline Keelean & Qilin, an animal in fiction. \\
\hline Khondai & A process of preparing warp thread \\
\hline Kleab kao & A process of preparing heddle shaft \\
\hline Krang & Stick lac. It is used for making red colour tone. \\
\hline \multirow[t]{2}{*}{ Kreng jai } & Kreng jai (being considerate) is a common \\
\hline & interpersonal behaviour ideal of Thais. \\
\hline \multirow[t]{2}{*}{ Kun } & Golden shower tree is the national flower of \\
\hline & Thailand \\
\hline
\end{tabular}


Mae

Makleo

Mai pen rai

Mudmee

Mugchatee

Mugga

Nirodha

Nuay

Oey

On nom thom ton

Phasin

Phee $\mathrm{Pu}$ Ta

Phee Ta Hake

Pook Siew

Prom vihan

Pun rod

Ruk sa naa

Saifon

Samol

Samudaya

Sao

Siew

Su Khwan

Thong leang

Thran Panya

Tine jong
It means mother but it is used as a title before name of Isan women who are married

Ebony fruit

Does not matter or no worries

Weft pattern cloth (see appendix B)

Annato fruit

The way leading to the cessation

The cessation of suffering

Unit is a design for float pattern weaving

Elder sister

Humility

Simple tube skirt

Ancestor spirit that is located at a community to take care of community members

(Isangate.com, 2007)

Paddy spirit that is located at paddy fields to take care of cultivation (Baan Maha.com, 2007)

Pook siew ceremony is considered as real friend declaration ceremony through a blessing ceremony

Dhamma principle

A process of preparing tube thread

Face saving

Cloth woven with mudmee weft.

Black Myrobalan or Chebulic Myrobalan

Cause of suffering

Miss

Friend

A blessing ceremony

A vertical string-heddle shaft

River wisdom

Cloth pattern made by embroidery weaving technique 
Tung

Upasampada

Yaka

Yo

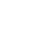

A ceremonial banner

Ordination rite

Grass leaves used for making roof

Noni (Morinda Citriforia). Young Noni leaves

are used as vegetables, and the fruits as herbal

medicine. 


\section{References}

Abu-Rashed, J., Bertaux, N. and Okunoye, A. (2005). Knowledge management and economic development in developing countries: an examination of the main enablers. Global Business and Economics Review, 7(1), 85-99.

Agostino, J. (2005). Narrative methods in business research: Some notes. Retrieved 25 October 2005, from http://www.globalresearchbusiness.com/methods/narrativeresearch.php?PHP SESSID=107adaf0146014ac5491db23affd377b.

Albino, V., Garavelli, A.C. and Schiuma, G. (1999). Knowledge transfer and interfirm relationships in industrial districts: The role of the leader firm. Technovation, 19, 53-63.

Al-Hawamdeh, S. (2003). Knowledge management: Cultivating knowledge professionals. Oxford: Chandos Publishing.

Alavi, M. and Leidner, D.E. (2001). Review: Knowledge management and knowledge management systems: Conceptual foundations and research issues. MIS Quarterly, 25(1), 107-136.

Aldridge, J.W. Jr. (2002). A multidimensional model for building knowledge assets: Applying socio-technical systems to online action research. Unpublished doctoral dissertation. Fielding Graduate Institute, Santa Barbara, CA, USA.

Alsup, M. and Strong, K. (2004). Enterprise content management: Charting the path. AIIM E-Doc magazine, 18(3), 16-27.

Alvesson, M. (1993). Cultural perspectives on organisations. Cambridge: Cambridge University Press.

Amabile, T.M. (1983). The social psychology of creativity. New York: SpringerVerlag.

Amabile, T.M. (1993). Motivational synergy: Toward new conceptualizations of intrinsic and extrinsic motivation in the workplace. Human Resource Management Review, 3(3), 185-201.

Amin, N. (2002). The informal sector in Asia from the decent work perspective. Employment paper 2002/4: Working paper on the informal economy. Retrieved 10 January 2005, from http://www.ilo-mirror.cornell.edu/ public/english/employment/infeco/download/assiaain.pdf

Anthony, W.P. and Gales, L.M. (2003). Organization theory: A strategic approach. ( $6^{\text {th }}$ ed.). Upper Saddle River, NJ.: Prentice Hall.

Argyris, C. and Schon, D.A. (1996). Organizational learning II: Theory, method, and practice. Reading, Massachusetts: Addison-Wesley Publishing Company.

Aristotle. (2002). Aristotle: Nicomachean ethics (C. Rowe, Tran.). Oxford: University press.

Ashforth, B.E. and Mael, F. (1989). Social Identity theory and the organization. The Academy of Management Review, 14(1), 20-39.

Atkinson, P. and Hammersley, M. (1994). Ethnography and participant observation. In N.K. Denzin and V.S. Lincoln (Eds.), Handbook of qualitative research (pp. 248-261). London: SAGE Publications.

Atmiyanandana, V, and Lawler, J.J. (2003). Culture and management in Thailand. In M. Warner (Ed.), Culture and management in Asia (pp. 228-248). London: Routledge Curzon.

Audi, R. (2003). Epistemology: A contemporary introduction to the theory of knowledge $2^{\text {nd }}$. New York: Routledge. 
Baan Maha Community. (2006). การผูกเสียว(เพื่อนแท้) [True friend ceremony]. Retrieved 30 July 2007, from http://www.baanmaha.com/forums/index.php?topic=1832.msg7157

Babbie, E. (2001). The practice of social research $\left(9^{\text {th }}\right.$ ed.). Belmont, CA.: Wadsworth.

Badaracco, J.L. Jr. (1991). The knowledge link. Boston: Harvard Business School Press.

Baek, S., Liebowitz, J., Prasad, S.Y. and Granger, M. (1999). Intelligent agents for knowledge management - Toward intelligent web-based collaboration within Virtual teams. In J. Liebowitz (Ed.), Knowledge management handbook (pp. 11-23). Boca Raton; CRC Press.

Balasubramanian, P., Nochur, K., Henderson, J.C. and Kwan, M.M. (1999). Managing process knowledge for decision support. Decicision Support Systems, 27(1), 145.

Baltes, P.B. (1993). The aging mind: Potential and limits. The Gerontologist, 33(5), 580-594.

Bartol, K.M. and Srivastava, A. (2002). Encouraging knowledge sharing: The role of organizational reward systems. Journal of Leadership \& Organizational Studies, 9(1), 64-76.

Barton, C. (1997). Micro enterprise business development services: Defining institutional options and indicators of performance. Retrieved 11 January 2005, from

http://www.knowledgemanagement.ittoolbox.com/browse.asp?c=KMPeerPu blishing\&r=\%2Fpub\%2FSH12803\%2Epdf

Belcher, J.R. (1994). Understanding the process of social drift among the homeless: A qualitative analysis. In E. Sherman and W. Reid (Eds.). Qualitative research in social work (pp. 126-134). New York: Columbia University Press.

Bernard, A.K. (1984). Report on the Southeast Asian NGO meeting, Singapore, May 1984. Retrieved 4 August 2007, from http://idrc.ca/archive/corpdocs/105428/105428.txt

Bertaux, D. (1981). From the life-history approach to the transformation of sociological practice. In D. Bertaux (Ed.), Biography and society: The life history approach in the social sciences, (pp. 29-46). Beverly Hills: SAGE Publications.

Bhatt, G.D. (2001). Knowledge management in organizations: examining the interaction between technologies, techniques, and people. Journal of Knowledge Management, 5(1), 68.

Bielawski, L. and Boyle, J. (1997). Electronic document management systems: A user centered approach for creating, distributing and managing online publications. Prentice Hall.

Black, M.E. (1980). The key to weaving: A textbook of hand weaving for the beginning weaver. New York: Macmillian Publishing.

Blackler, F. (2002). Knowledge, knowledge work, and organizations. In C.W. Choo and N. Bontis (Eds.), The strategic management of intellectual capital and organizational knowledge (pp. 47-64). New York: Oxford University Press.

Bloor, M. (1997). Addressing social problems through qualitative research. In D. Silverman (Ed.), Qualitative research theory, method and practice (pp. 221238). London: SAGE Publications.

Boje, D.M. (2001). Narrative methods for organizational and communication research. London: SAGE Publications. 
Boonmathya, R.T. (2003). A narrative of contested views of development in Thai society: Voice of villagers in rural northeastern Thailand. Southeast Asian Studies, 41(3), 269-298.

Bourdieu, P. (1986). The forms of capital. In J.G. Richardson (Ed.), Handbook of theory and research for the sociology of education (pp.239-258). Westport: Greenwood Press.

Brown, J.S. and Duguid, P. (1998). Organizing knowledge. California Management Review, 40(3), 90-111.

Bryman, A. (2004). Social research methods. New York: Oxford University Press.

Buasri, U. (1983). วัฒนธรรมอีสาน: 413123 [Isan culture: 413 123]. Khon Kaen, Thailand: Khon Kaen University, Faculty of Humanities and Social Sciences.

Buddhadasa.com. (2008). อิทธิบาท 4 [4-iddhipatihariya]. Retrieved 10 January 2008. from http://www.buddhadasa.com/rightstudydham/itibath4.html

Burke, G.L. (1970). The growth and nature of urban areas in developing countries. In Nutritional problem of urban communities symposium: Vol. 29. (pp.157164).

Burt, R.S. (1992). Structure holes: The social structure of competition. Cambridge: Harvard University Press.

Capello, R. and Faggian, A. (2005). Collective learning and relational capital in local innovation processes. Regional Studies, 39(1), 75-87.

Carrillo, P.M., Robinson, H.S., Anumba, C.J. and Bouchlaghem, N.M. (2006). A knowledge transfer framework: The PFI context. Construction Management and Economics, (24), 1045-1056.

Cassell, C.and Symon, G. (1994). Qualitative research in work contexts. In C. Cassell and G. Symon (Eds.), Qualitative methods in organizational research: A practical guide (pp. 1-13). London: SAGE Publications.

Castellano, M.B. (2000). Updating aboriginal traditions of knowledge. In G.J. Sefa Dei, B.L. Hall, and D.G. Rosenberg (Eds.), Indigenous knowledge in global contexts (pp.21-36).Toronto: University of Toronto Press.

Caulkins, D.D. (2004). Identifying culture as a threshold of shared knowledge: A consensus analysis method. International Journal of Cross Cultural Management, 4(3), 317-333.

Chatman, S. (1978). Story and discourse: Narrative structure in fiction and film. London: Cornell University Press.

Chattell, A. (1998). Creating value in the digital era achieving success through insight, imagination and innovation. Wiltshire: Macmillan Business.

Choo, C.W. (1998). The knowing organization: How organizations use information to construct meaning, create knowledge, and make decisions. New York: Oxford University Press.

Christensen, K.S. and Bukh, P.N. (2005). Knowledge Management: Two perspectives. In P.N. Bukh, K.S. Christensen and J. Mouritsen (Eds.), Knowledge Management and Intellectual Capital Establishing a Field of Practice (pp. 15-34). New York: Palgrave Macmillan.

Clandinin, D.J. and Connelly, F.M. (2000). Narrative inquiry experience and story in qualitative research. San Francisco: Jossey-Bass Publishers.

Cohen, W.M. and Levinthal, D.A. (1990). Absorptive capacity: A new perspective on learning and innovation. Administrative Science Quarterly, 35(1), 128-152.

Coleman, J.S. (1988). Social capital in the creation of human capital. The American Journal of Sociology, 94, S95-S120. 
Collison, C. and Parcell, G. (2004). Learning to fly: Practical knowledge management from leading and learning organizations $\left(2^{\text {nd }}\right.$ ed.). Chichester: Capstone Publishing Ltd.

Community enterprise development center in Thailand. (2001). The opinions on action plan of SMEs promotion. Bangkok: Department of Commerce and Accounting, Thammasat University.

Conle, C. (2000). Narrative inquiry: Research tool and medium for professional development. European Journal of Teacher Education, 23(1), 49-63.

Connell, N., Klein, J.H. and Powell, P.L. (2003). It's tacit knowledge but not as we know it: Redirecting the search for knowledge. Journal of the Operational Research Society, (54), 140-152.

Conning, J. (1999). Outreach, sustainability and leverage in monitored and peermonitored lending. Journal of Development Economics. 60, 51-77.

Conway, S. (1992). Thai textiles. London: British Museum Press.

Creswell, J.W. (2005). Educational research: Planning, conducting, and evaluating quantitative and qualitative research ( $2^{\text {nd }}$. Ed.). Upper Saddle River, NJ.: Merrill Prentice Hall.

Czarniawska, B. (1998). A narrative approach to organization studies. Thousand Oaks, California: SAGE Publications.

Dalkir, K. (2005). Knowledge management in theory and practice. Burlington, MA: Elsevier Butterworth-Heinemann.

Datta, R. (2000). On their own: Development strategies of the Self-Employed Women's Association (SEWA) in India. The Society for International Development, 43(4), 51-55.

Davenport, T.H., De Long, D.W. and Beers, M.C. (1997). Building successful knowledge management projects. Retrieved 2 November 2005, from http://www.providersedge.com/docs/km_articles/Building_Successful_KM_ Project.pdf.

Davenport, T.H. and Prusak, L. (1998). Working knowledge: How organizations manage what they know. Boston: Harvard Business School Press.

Davison, P. and Honig, B. (2003). The role of social and human capital among nascent entrepreneurs. Journal of Business Venturing, 18, 301-331.

De Long, D.W. and Fahey, L. (2000). Diagnosing cultural barriers to knowledge management. Academy of Management Executive, 14(4). 113-127.

Delamont, S. (2004). Ehtnography and participant observation. In C. Seale, G. Gobo, J. F. Gubrium and D. Silverman (Eds.), Qualitative research practice (pp. 217-229). London: SAGE Publications.

Denison, D.R. and Mishra, A. K. (1995). Toward a theory of organizational culture and effectiveness. Organization Science, 6(2), 204-223.

Despres, C. and Chauvel, D. (2000). A thematic analysis of the thinking in knowledge management. In C. Despres and D. Chauvel (Eds.), Knowledge horizons: The present and the promise of knowledge management (pp.55-86). Oxford: Butterworth-Heinemann.

Dey, I. (1993). Qualitative data analysis: A user-friendly guide for social scientists. London: Routledge.

Dixon, N.M. (1999). The changing face of knowledge. The Learning Organization, 6(5), 212.

Dooley, K.J., Corman, S.R. and McPhee, R.D. (2002). A knowledge directory for identifying experts and areas of expertise. Human Systems Management, (21), 217-228. 
Drisko, J.W. (1997). Strengthening qualitative studies and reports: Standards to promote academic integrity. Journal of Social Work Education, 33(1), 185197.

Driver, M. (2002). The learning organization: Foucauldian gloom or Utopian sunshine? Human Relations, 55(1), 33-53.

Dubey-Villinger, N. (2001). Thai business culture: Hierarchy and groups, initiative and motivation. In J.B. Kidd, X. Li and F. Richter (Eds.), Advances in human resource management in Asia (pp. 105-116). New York: Palgrave.

Durkin, J. (1994). Expert systems: Design and development. New York: Macmillan Publishing Company.

Earl, M. (1997). Knowledge as strategy. In L. Prusak (Ed.), Knowledge in organizations (pp. 1-16). Newton: Butterworth-Heinemann.

Elliott, J. (2005). Using narrative in social research qualitative and quantitative approaches. London: SAGE.

Emory, C.W. and Cooper, D.R. (1991). Business research methods (4 ${ }^{\text {th }}$ ed.). Boston: IRWIN.

Fetterman, D.M. (1991). A walk through the wilderness: learning to find your way. In W.B. Shaffir and R.A. Stebbins (Eds.), Expriencing fieldwork: An insider view of qualitative research (pp. 87-96). Newbury Park, CA: SAGE Publications.

Fischetti, D. (2000). Linking community organizing and adult learning to explore empowered learning. Unpublished doctoral dissertation. Temple University, Philadelphia, PA, USA.

Ford, D.P. and Chan, Y.E. (2003). Knowledge sharing in a multi-cultural setting: a case study. Knowledge Management Research \& Practice, 1, 11-27.

Francis, J. (2006). Managing marine protected areas: A toolkit for the Western Indian Ocean. Retrieved 20 April 2006, from http://www.wiomsa.org/mpatookit/Home.htm

Frechtling, J. and Sharp, L. (1997). User-friendly handbook for mixed method evaluations. Retrieved 27 January 2005, from http://www.ehr.nsf.gov/EHR/REC/pubs/NSF97-153/CHAP_3.htm

Gamble, P.R. and Blackwell, J. (2001). Knowledge management: A state of the art guide. London: Kogan Page.

Gamchoei, A. (2001). Community enterprise and community consciousness in forestry management: A case study of Ban Khaoroatienthong, Neankham subdistrict, Chainat province. Bangkok: Mahidol University.

Garud, R. (1997). On the distinction between know-how, know-why, and know-what. Advances in Strategic Management, 14, 81-101.

Garza, J.M. and Ibbs, C.W. (1992). Knowledge Elicitation strategies and experiments applied to construction. In Arciszewski, T. and Rossman, L. (Eds.). Knowledge acquisition in civil engineering. (pp. 69-85) New York, NY: American Society of Civil Engineering.

Geertz, C. (1973). The interpretation of cultures: Selected essays. London: Fontana.

Gibbs, G.R. (2002). Qualitative data analysis: Explorations with NVivo. Buckingham: Open University Press.

Glesne, C. (1999). Becoming qualitative researchers: An introduction ( $2^{\text {nd }}$ ed.). New York: Addison Wesley Longman.

Goffee, R. and Jones, G. (1999). What holds the modern company together? In Harvard business review on managing people (pp. 1-44). Boston: Harvard Business School Publishing. 
Gopalakrishnan, S. and Santoro, M.D. (2004). Distinguishing between knowledge transfer and technology transfer activities: The role of key organizational factors. IEEE Transactions on Engineering Management, 51(1), 57-69.

Gorman, G.E. and Clayton, P. (2005). Qualitative research for the information professional: A practical handbook ( $2^{\text {nd }}$ ed.). London: Facet Publishing.

Grant, R.M. (1996). Toward a knowledge-based theory of the firm. Strategic Management Journal, 17, 109-122.

Gray, B. (1998). Collaboration The constructive management of differences. In G.R. Hickman (Ed.). Leading organizations: Perspectives for a new era (pp.467480). Thousand Oaks: SAGE Publications.

Gray, V., Kelly, T. and Minges, M. (2002). Bits and bahts: Thailand internet case study. Retrieved 21 April 2006, from http://www.itu.int/ITUD/ict/cs/thailand/material/THA\%20CS.pdf

Grenier, L. (1998), Working with indigenous knowledge: A guide for researchers. Ottawa: International Development Research Centre.

Guba, E.G. and Lincoln, Y.S. (1994). Completing paradigms in qualitative research. In N.K. Denzin and V.S. Lincoln (Eds.). Handbook of qualitative research, (pp. 105-117). London: SAGE Publications.

Gubrium, J.F. and Holstein, J.A. (1997). The new language of qualitative method. New York: Oxford University Press.

Gubrium, J.F. and Holstein, J.A. (1998). Narrative practice and the coherence of personal stories. The Sociological Quarterly, 39(1), pp. 163-187.

Gudmundsdottir, S. (1995). The narrative nature of Pedagogical content knowledge. In H. McEwan and K. Egan (Eds.), Narrative in teaching, learning, and research (pp.24-38). New York: Teachers College Press.

Gudmundsdottir, S. (1996). The teller, the tale, and the one being told: The narrative nature of the research interview. Curriculum Inquiry, 26(3), 293-306.

Gupta, A.K. and Govindarajan, V. (2000a). Knowledge management's social dimension: Lessons from Nucor Steel. MIT Sloan Management Review, 42(1), 71-79.

Gupta, A.K. and Govindarajan, V. (2000b). Knowledge flows within multinational corporations. Strategic Management Journal, 21(4), 473.

Gupta, B., Iyer, L.S. and Aronson, J.E. (2000). Knowledge management: Practices and challenges. Industrial Management + Data Systems, $100(1), 17$.

Gupta, J.N.D., Sharma, S.K., and Hsu, J. (2004). An overview of knowledge management. Creating knowledge based organizations. Hershey, Idea Group.

Hall, I. and Hall, D. (2004). Evaluation and social research: Introducing small-scale practice. New York: Palgrave Macmillan.

Hall, R. H. (1982). Organizations structure and process (3 ${ }^{\text {rd }}$ ed.). Englewood Cliffs, New Jersey: Prentice-Hall.

Hall, R. (1992). The strategic analysis of intangible resources. Strategic Management Journal, 13(2), 135-144.

Halvorson-Quevedo, R. (1991). The growing potential of micro-enterprises. Organisation for economic cooperation and development, 173, 7-11.

Hampden-Turner, C. (1990). Corporate culture from vicious to virtuous circles. London: Hutchinson.

Handy, C. (1995). Managing the dream. In S. Chawla and J. Renesch (Eds.), Learning organizations: Developing cultures for tomorrow's workplace (pp. 44-54). Portland, Oregon: Productivity Press. 
Handzic, M. and Agahari, D. (2004). Knowledge sharing culture: A case study. Journal of Information \& Knowledge Management, 3(2), 135-142.

Hansen, M.T., Nohria, N. and Tierney, T. (1999). What's your strategy for managing knowledge? Harvard Business Review, 106-116.

Hansen, S.A. and VanFleet, J.W. (2003). Traditional knowledge and intellectual property: A Handbook on issue and options for traditional knowledge holders in protecting their intellectual property and maintaining biological diversity. Washington, DC: AAAS.

Hantrakul, S. (2000). Success factors for community enterprise operation: A case study of rice mill industries in south northeast region. Bangkok: Kasetsart University.

Harris, P.R. and Moran, R.T. (1996). Managing cultural differences ( $4^{\text {th }}$ ed.). Houston, Texas: Gulf Publishing Company.

Hickins, M. (1999). Xerox shares its knowledge. Management Review, 88(8), 40-45.

Hirschheim, R. and Klein, H.K. (1989). Four paradigms of information systems development. Communications of the ACM, 32(10), 1199-1216.

Hislop, D. (2005). Knowledge management in organizations A critical introduction. Oxford: Oxford University Press.

Hoffman, J.J., Hoelscher, M.L. and Sherif, K. (2005). Social capital, knowledge management, and sustained superior performance. Journal of Knowledge Management, 9(3), 93-100.

Hofstede, G. (1980). Culture's consequences: International differences in workrelated values. Beverly Hills, California: Sage Publications.

Hofstede, G. (1991). Cultures and organizations: Software of the mind: Intercultural cooperation and its importance for survival. London: McGraw-Hill.

Holmes, H., Tangtongtavy, S. and Tomizawa, R. (1997). Working with the Thais: A guide to managing in Thailand ( $7^{\text {th }}$ ed.). Bangkok: White Lotus Co., Ltd.

Holsapple, C.W. and Joshi, K.D. (1999). Knowledge selection: Concepts, issues, and technologies. In J. Liebowitz (Ed.), Knowledge management handbook (pp. 7-17). Boca Raton; CRC Press.

Holtshouse, D. (1999). Ten knowledge domains: Model of a knowledge-driven company? Knowledge and Process Management, 6(1), 3-8.

Hudson, M. (1995). Managing without profit: The art of managing third-sector organizations. London: Penguin.

Huizing, A. and Bouman, W. (2002). Knowoledge and learning, marketing and organization. In C.W. Choo and N. Bontis (Eds.), The strategic management of intellectual capital and organizational knowledge (pp.185-204). New York: Oxford University Press.

Hunter, M.G. (2006). Experiences conducting cross-cultural research. Journal of Global Information Management, 14(2), 75-89.

Hutanuwatr, N. and Hutanuwatr, N. (2002). 10 years Pare Pun group: Toward hand weaving community enterprise. Bangkok: The Office of Community Fund.

Hylko, J. (2005). Thanks for the memories: Capturing expert knowledge. Power, 148(4). 58.

Hylton, A. (2002). Knowledge management is not new to small companies. Retrieved 26 October 2004,

from:http://www.knowledgemanagement.ittoolbox.com/browse.asp?c=KMP eerPublishing\&r=\%2Fpub\%2FSH12803\%2Epdf

IBM. (2005). About IBM. Retrieved 24 April 2005, from http://www.ibm.com/ibm/th 
ILO. (2000a). Skills development for the informal economy: Who are they? Retrieved 25 December 2004, from http://www.ilo.org/public/english/employment/ skills/informal/who.htm

ILO. (2000b). Gender issues in micro-enterprise development. Retrieved 3 March 2006, from http://www.ilo.org/public/english/employment/ ent/papers/gender.htm

Jackson, T. (1993). Organizational behaviour in international management. Oxford: Butterworth-Heinemann

Jasimuddin, S.M. (2007). Exploring knowledge transfer mechanisms: The case of a UK-based group within a high-tech global corporation. International Journal of Information Management, 27, 294-300.

Jommuang, L. (1997). Role of community enterprise in community consciousness in community forest management: A case study of Silalang subdistrict, Pua district, Nan province. Retrieved 15 December 2004, from http://thesis.tiac.or.th.

Jones, P.C. (1964). The nature of knowledge. New York: The Scarecrow Press.

Kankanhalli, A., Tanudidjaja, F., Sutanto, J. and Tan, B.C.Y. (2003). The roles of IT in successful knowledge management initiatives. Communications of ACM, 46(9), 69-73.

Katetade, B. (1997). การศึกษามานุษยวิทยาวัฒนธรรมในภาคอีสาน [A study of cultural anthropology in Isan region]. Mahasarakham, Thailand: Mahasarakham University, Anthropology and Social Science faculty, Department of Thai Language and East Languages.

Keong, L.C. and Al-Hawamdeh, S. (2002). Factors impacting knowledge sharing. Journal of Information \& Knowledge Management, 1, 49-56.

Kilgour, M. (2006). Improving the creative process: Analysis of the effects of divergent thinking the techniques and domain specific knowledge on creativity. International Journal of Business and Society,7(2), 79-107.

Kirk, J. and Miller, M.L. (1986). Reliability and validity in qualitative research. London: SAGE Publications.

Kitahara, A. (2000). Development prospects of small industry in Thailand. In M.A. Abdullah and M.I. Bakar (Eds.), Small and medium enterprises in Asian Pacific countries (Vol.3, pp.73-90). New York: Nova Science Publishers.

Kitaro, N. (1960). A study of good. Translated by V.H. Vigllelmo. New York: Greenwood Press.

Kluckhohn, C. (1962). The concept of culture. In R. Kluckhohn (Ed.), Collected essays of Clyde Kluckhohn: Culture and behavior (pp.19-73). New York: The Free Press of Glencoe.

Knowledge Management Institute (KMI). (2005a). Ko Koa Kao Knowledge management for Nan civil. Bangkok: Knowledge Management Institute.

Knowledge Management Institute (KMI). (2005b). Knowledge management model in Thai context. Retrieved 9 June 2005, From http://www.kmi.or.th/document/ model_km47.pdf.

Koanantakool, T. (2003). ICT and community building in the rural areas of Thailand. Retrieved 21 April 2006, from http://www.pecc.org/PECC2003Brunei/cbfworkshop/session1-thaweesak.pdf.

Komin, S. (1990). Culture and work-related values in Thai organizations. International Journal of Psychology, 25(5-6), 681-704.

Kotkanta, M. (2006). ปู่ตาและผีตาแฮก [Ancestor spirit and paddy field spirit]. Retrieved 5 August 2007, from http://www.isangate.com/local/tahag.htm 
Kotter, J.P. and Heskett, J.L. (1992). Corporate culture and performance. New York: Maxwell Macmillan International.

Kovan, J.T. (2001). Sustaining passion: The experience of being an environmentalist in a small nonprofit organization. Unpublished doctoral dissertation. Michigan State University, East Lansing, MI, USA.

Kritsanaphathi, W. and Kritsanaphathi, W. (1983). การประเมินผลการผูกเสี่ยว : ศึกษาเฉพาะกิ่งอำเภอ เปือยน้อย จังหวัดขอนแก่น [The evaluation in real friend ceremony: A case study of Peoy sub-district, Khon Kaen province] Khon Kaen University

Kroma, S. (1995). Popularizing science education in developing countries through indigenous knowledge. Retrieved 20 April 2006, from http://www.nuffic.nl/ciran/ikdm/3-3/articles/kroma.html

Kwan, M.M. and Cheung, P. (2006). The knowledge transfer process: From field studies to technology development. Journal of Database Management, 17(1), $16-32$.

Lam, A. (1997). Embedded firms, embedded knowledge: Problems in collaboration and knowledge transfer in global cooperative ventures. Orgabuzation Studies, 18(6), 973-996.

Lam, A. (2000). Tacit knowledge, organizational learning and societal institutions: An integrated framework. Organization Studies, 21(3), 487-513.

Langill, S. (2007, February). Introduction to indigenous knowledge. The Overstory Agroforestry Ejournal, Article 82. Retrieved 21 July 2007, from http://www.agroforestry.net/overstory/overstory82.html

LearnTripitaka.com. (2005). Dhamma principles. Retrieved 22 July 2005, from http://www.learntripitaka.com/index.html

Lee, J. and Kim, Y. (2001). A stage model of organizational knowledge management: A latent content analysis. Expert Systems with Applications, 20, 299-311.

Lemon, M. and Sahota, P.S. (2005). Organizational culture as a knowledge repository for increased innovative capacity. Retrieved 1 June 2005, from http://is.lse.ac.ulc/Support/OKLC_2002/pdf_files/ID88.pdf

Lesser, E. and Prusak, L. (1999). White paper: Communities of practice, social capital and organizational knowledge. Retrieved 27 July 2005, from http://openacademy.mindef.gov.sg/openacademy/central/html\%20folder/km/ bcp/downloads/copsc3.pdf.

Levine, J.M. and Moreland, R.L. (1999). Knowledge transmission in work groups: Helping newcomers to succeed. In L. Thompson, J. M. Levine and D.M. Messick (Eds.), Shared cognition in organizations: The management of knowledge (pp.267-296). Mahwah, NJ: Lawrence Erlbaum Associates.

Liebowitz, J. (2001). Knowledge management: Learning from knowledge engineering. (pp. 75 - 92). Boca Raton; CRC Press.

Lin, N. (2001). Social capital: A theory of social structure and action. Cambridge: Cambridge University Press.

Litan, R.E. (2005). Information technology meets the developing world. International Studies Review, 7, 326-327.

Littrell, M.A. and Dickson, M.A. (1997). Alternative trading organizations: Shifting paradigm in a culture of social responsibility. Human Organization, 56(3), 344-352. 
Lopez, S.P., Peon, J.M.M. and Ordas, C.J.V. (2004). Managing knowledge: The link between culture and organizational learning. Journal of Knowledge Management, 8(6), 93-104.

Martin, L.M. and Matlay, H. (2003). Innovative use of the Internet in established small firms: The impact of knowledge management and organizational learning in accessing new opportunities. Qualitative Market Research, 6(1), 18

Maslow, A.H. (1987). Motivation and personality. New York: Harper and Row.

Mason, J. (1996). Qualitative researching. London: SAGE Publications.

Maxwell, J.A. (1992). Understanding and validity in qualitative research. Harvard Educational Review, 62(3), 279-299.

Maxwell, J.A. (1996). Qualitative research design: An interactive approach. Thousan Oaks, SAGE Publications.

Maxwell, R. (2003). Textiles of Southeast Asia Tradition, trade and transformation. Singapore: Periplus.

McDermott, R. and O’Dell, C. (2001). Overcoming cultural barriers to sharing knowledge. Journal of Knowledge Management, 5(1), 76.

Miles, M.B. and Huberman, A.M. (1994). Qualitative data analysis: An expanded sourcebook ( $2^{\text {nd }}$ ed. $)$. Thousand Oaks, California: Sage.

Miller, G. (1997). Contextualizing texts: Studying organizational texts. In G. Miller and R. Dingwall (Eds.). Context and Method in Qualitative Research, pp.7791. Thousand Oaks, SAGE Publications.

Mishler, E.G. (1986). Research Interviewing Context and Narrative. Massachusetts: Harvard University Press.

Mitchell, H. (2005). Knowledge sharing - The value of story telling. International Journal of Organizational Behaviour, 9(5), 632-641.

Moffett, S., McAdam, R. and Parkinson, S. (2002). Developing a Model for technology and cultural factors in knowledge management: A factor analysis. Knowledge and Process Management, 9(4), 237- 253.

Moffett, S., McAdam, R. and Parkinson, S. (2004). Technological utilization for knowledge management. Knowledge and Process Management, 11(3), 175184.

Moorman, C. and Miner, A.S. (1998). Organizational improvisation and organizational memory. The Academy of Management Review, 23(4), 698723.

Myers, M.D. (1997). Qualitative research in information systems. Retrieved 27 September 2005, from http://www.qual.auckland.ac.nz

Mwaisela, F.A. (2000). WTO and Sustainable Seed Multiplication Programmes in Tanzania. The Society for International Development, 43(2), 83-87.

Nahapiet, J. and Ghoshal, S. (1998). Social capital, intellectual capital, and the organizational advantage. Academy of Management, 23(2), 242-266.

Nakano, R. (2002). Web content management A collaborative approach. Boston; Addison-Wesley.

Narintharangkul-na-Ayuthaya, P. (2002). Development trend of community economic institute in up-land area. In N. Petprasert and P. Wongkul (Eds.), Community enterprise: วิสาหกิจชุมชน: กลไกเศรษฐิิฐฐานราก [Community enterprises: Economic mechanism for grass root] (pp. 107-129). Bangkok: Edison Press Production.

Nartsupa, C. and Lertwicha, P. (1994). วัฒนธรรมหมู่บ้านไทย [Thai village culture]. Bangkok: Sangsan Publishing. 
National Economic and Social Development Board. (2005). Population data. Retrieved 12 January 2006, from, http://social.nesdb.go.th/nesdbsoc/modules/report/rpsocial. aspx?id=rppop\&group=rpsocial

National Electronics and Computer Technology Center (NECTEC). (2003). Thailand ICT indicators moving towards the information society. Bangkok: NECTEC.

Nelson, R.E. (1987). Issues and trends in small enterprise promotion. In International Labour Office (Ed.), Small enterprise development: Policies and programmes ( $2^{\text {nd }}$ ed.) (pp. 251-266). Geneva: International Labour Office.

Newell, S., Robertson, M., Scharbrough, H. and Swan, J. (2002). Managing Knowledge Work. New York: Palgrave.

Nilakanta, S., Miller, L.L. and Zhu, D. (2006). Organizational memory management: Technological and research issues. Journal of Database Management, 17(1), 85-94.

Nonaka, I. (1994). A dynamic theory of organizational knowledge creation. Organization Science, 5(1), 14-37.

Nonaka, I. (2002). A dynamic theory of organizational knowledge creation. In C.W. Choo and N. Bontis (eds.), The strategic management of intellectual capital and organizational knowledge (pp. 437-462). New York: Oxford University Press.

Nonaka, I. and Takeuchi, H. (1995). The knowledge-creating company. New York: Oxford University Press.

Noyshop.com (2004). แหล่งท่องเที่ยวธรรมชาติ [Eco-tours]. Retrieved 22 June 2008, from http://www.noyshop.com/Noy/luangprabang6.php.

O’Callaghan, C.M. (1998). Social construction of preservice teachers' instructional strategies for reading. Unpublished doctoral dissertation. Fordham University, New York, NY, USA.

O’Connor, T. (2005). Qualitative social science research methodology. Retrieved 27 January 2005, from http://faculty.ncwc.edu/toconnor/308/308lect09.htm

Obomsawin, R. (2000). Indigenous knowledge and sustainable development. Development Express, (3), 1-3.

Olivera, F. and Argote, L. (1999). Organizational learning and new product development: CORE processes. In L. Thompson, J.M. Levine and D.M. Messick (Eds.), Shared cognition in organizations: The management of knowledge (pp.297-326). Mahwah, NJ: Lawrence Erlbaum Associates.

Ott, J.S. (1989). The organizational culture perspective. Chicago: The Dorsey Press.

Pamapimai (2001). Community enterprises: A case study of the Thai noodle group at Ban Pradoak, Meanwai subdistrict, Muang district, Nakonratchasima province. Bangkok: Mahidol University.

Pan, S.L. and Scarbrough, H. (1999). Knowledge management in practice: An exploratory case study. Technology Analysis \& Srategic Management, 11(3), 359-374.

Paton, R. and Cornforth, C. (1992). What's different about managing in voluntary and non-profit organizations? In J. Batsleer, C. Cornforth and R. Paton (Eds.), Issues in voluntary and non-profit management (pp.36-46). Wokingham: Addison-Wesley Publishing Company.

Patriotta, G. (2004). On studying organizational knowledge. Knowledge Management Research \& Practice, (3), 3-12.

Patton, M.Q. (2002). Qualitative evaluation and research methods. Newbury Park, Calif.: Sage Publications. 
Pealnain, L. (1998). Roles of women in community enterprise development: A case study of the herbal shampoo group at Ban Thasai, Moo 1, Bangluang subdistrict, Subpphaya district, Chainat province. Bangkok: Mahidol University. Bangkok: Office of Research Support Fund.

Pemberton, J.D. and Stonehous, G.H. (2000). Organizational learning and knowledge assets. The Learning Organization, 7(4), 184-193.

Perakyla, A. (1997). Reliability and validity in research based on tapes and transcripts. In P. Silverman (Ed.). Qualitative Research Theory, methods and practice, (pp. 201-220). London: SAGE Publications.

Personal Narratives Group. (1989). Interpreting women's lives: Feminist theory and personal narratives. Indianapolis: Indiana University Press.

Petprasert, N. (1997). Feasibility study of community enterprise establishment. Bangkok: Office of Research Support Fund.

Petprasert, N. and Wongkul, P. (2002). In N. Petprasert and P. Wongkul (Eds.), Community enterprise: วิสาหกิจชุมชน: กลไกเศรษฐิิฐานราก [Community enterprises: Economic mechanism for grass root]. (pp. 1-11). Bangkok: Edison Press Production.

Prusak, L. (2001). Where did knowledge management come from? IBM Systems Journal, 40(4), 1002-1007.

Punch, M. (1994). Politics and ethics in qualitative research. In N.K. Denzin and Y.S. Lincoln (Eds.), Handbook of qualitative research, (pp. 83-97). London: SAGE Publications.

Punch, K.F. (2005). Introduction to social research quantitative and qualitative approaches $\left(2^{\text {nd }}\right.$ ed.). London: SAGE Publications.

Rasheed, N. (2005). The impact of knowledge management on SMEs. Retrieved 7 July 2005, from: http://www.knowledgeboard.com/download/5930/THEIMPACT-of-KM-ON-SMEs.pdf

Rao, S.S. (2006). Indigenous knowledge organization: An Indian scenario. International Journal of Information Management, 26, 224-233.

Reid, F. (2003). Creating a knowledge-sharing culture among diverse business units. Employment Relations Today, 30, 43-49.

Reigle, R.F. (2001). Measuring organic and mechanistic cultures. Engineering Management Journal, 13(4), 3-8.

Ribiere, V.M. and Sitar, A.S. (2003). Critical role of leadership in nurturing a knowledge-supporting culture. Knowledge Management Research \& Practice, 1, 39-48.

Riessman, C.K. (1993). Narrative analysis. Newbury Park, California: SAGE Publications.

Roberts-Witt. S.L. (2005). A "eureka!" moment at Xerox. Retrieved 30 July 2005, from http://www.pcmag.com/article2/0,4149,28792,00.asp

Rogers, E.M. (1995). Diffusion of innovations, (4 ${ }^{\text {th }}$ ed.). New York: The Free Press.

Ryen, A. (2004). Ethical issues. In C. Seale, G. Gobo, J.F. Gubrium and D. Silverman (Eds.), Qualitative research practice (pp. 230-247). London: SAGE Publications.

Ryle, G. (1949). Concept of mind. London: Hutchinson’s University Library.

Ryu, C., Kim, Y.J., Chaudhury, A. and Rao, H. R. (2005). Knowledge acquisition via three learning processes in enterprise information portals: Learning-byinvestment, learning-by-doing, and learning-from-other. MIS Quarterly, 29(2), 245-278. 
Sackmann, S.A. (1991). Cultural knowledge in organizations: Exploring the collective mind. New Bury Park: SAGE Publications.

SAIC. (2005). KM \& British Petroleum: A historic example of the SAIC KM approach. Retrieved 7 July 2005, from http://www.saic.com/km/who.html

Sanchez, R. (1997). Managing articulated knowledge in competence-based competition. In Sanchez, R. and Heene, A. (Eds.), Strategic learning and knowledge management (pp. 163-187). Chichester: John Wiley \& Sons.

Scarbrough, H. and Carter, C. (2000). Investigating knowledge management. London: Chartered Institute of Personnel and Development.

Schein, E.H. (1992). Organizational culture and leadership. San Francisco: JosseyBass.

Schreiner, M. (2001). Microenterprise in the first and third worlds. Retrieved 11 January 2005, from ftp://wueconb.wustl.edu/econ-wp/dev/papers/ 0108/0108001.pdf.

Schultz, U. and Leidner, D.E. (2002). Studying knowledge management in information systems research: discourses and theoretical assumptions. MIS Quarterly, 26(3), 213-243.

Schwandt, T.A. (1994). Constructivist, interpretivist approaches to human inquiry. In N.K. Denzin and Y.S. Lincoln (Eds.), Handbook of qualitative research (pp. 118-137). Thousand Oaks, California: Sage Publications.

Sefa Dei, G.J., Hall, B.L. and Rosenberg, D.G. (2000). Introduction. In G.J. Sefa Dei, B.L. Hall, and D.G. Rosenberg (Eds.), Indigenous knowledge in global contexts (pp.3-17).Toronto: University of Toronto Press.

Sena, J.A. and Shani, A.B. (1999). Intellectual capital and knowledge creation: Towards an Alternative Framework. In J. Liebowitz (Ed.), Knowledge management handbook (pp. 8-16). Boca Raton; CRC Press.

Shama, M., Miller, H.G. and Reeder, R. (1990). Micro-enterprise growth: Operational models and implementation assistance in third and fourth world countries. Jounal of Small Business Management, 28, 9-21.

Shrubsole, J.L. (2003). Engagement: Public health nurses and Christian faith communities. Unpublished doctoral dissertation. Rush University, Chicago, Illinois, USA.

Silverman, D. (2005). Doing qualitative research: A practical handbook ( $2^{\text {nd }}$ ed.). London: SAGE Publications.

Skyrme, D.J. (1999). Knowledge networking: Creating the collaborative enterprise. Oxford: Butterworth-Heinemann.

Small, M.W. (2004). Wisdom and now managerial wisdom: do they have a place in management development programs? The Journal of Management Development, 23(8), 751-764.

Smircich, L. (1985). Is the concept of culture a paradigm for understanding organizations and ourselves? In P.J. Frost, L.F. Moore, M.R. Louis, C.C. Lundberg and J. Martin (Eds.), Organizational culture (pp. 55-72). Beverly Hills, California: Sage Publications.

Smith, H.A. and Mckeen, J.D. (2005). Instilling a knowledge-sharing culture. The Knowledge Management Forum, 2(4),

Sparrow, J. (2001). Knowledge management in small firms. Knowledge and Process Management, 8(1), 3-16.

Sparrow, J. (2005). Organisational learning in small firms: Implications for business support. Retrieved 5 August 2005, from: http://kmc.tbs.uce.ac.uk/ kmcpublications/Org\%20Learning\%20Small\%20Firms.pdf. 
Starbuck, W.H. (1992). Learning by knowledge-intensive firm. Journal of Management Studies, 29(6), 713-740.

Stata, R. (1996). Organizational learning: The key to management innovation. In K. Starkey (Ed.), How organizations learn (pp. 316-334). London: International Thomson Business Press.

Steinheider, B. and Al-Hawamdeh, S. (2004). Team coordination, communication and knowledge sharing in SMEs and large organizations. Journal of Information \& knowledge management, 3, $223-232$.

Sternberg, R. and Lubart, T.I. (1996). Investing in Creativity. American Psychologist, 51(7), 677-688.

Stewart, T.A. (1997). Intellectual capital: The new wealth of organizations. New York: Doubleday.

Storey, K. (2005). Geography 6250/4410 - Seminar in resource management environmental impact assessment. Retrieved 20 April 2006, from http://www.ucs.mun.ca/ kstorey/course.htm.

Sukula, S.K. (2006). Developing indigenous knowledge databases in India. The Electronic Library, 24(1), 83-93.

Sutton, D.C. (2001). What is knowledge and can it be managed? European Journal of Information Systems, (10), 80-88.

Sveiby, K. (2001). What is knowledge management? Retrieve December 2005, from http://www.sveiby.com/Portals/0/articles/KnowledgeManagement.html.

Sveiby, K. and Simons, R. (2002). Collaborative climate and effectiveness of knowledge work - an empirical study. Journal of Knowledge Management, 6(5), 420-433.

Swan, J., Newell, S., Scarbrough, H. and Hislop, D. (1999). Knowledge management and innovation: networks and networking. Journal of Knowledge Management, 3(4), 262.

Tajfel, H. (1978). The achievement of group differentiation. In H. Tajfel (Ed.), Differentiation between social groups: Studies in the social psychology of intergroup relations (pp. 77-98). London: Academic Press.

Talisayon, S.D. (2002). Knowledge and people. Business World, 1.

Tambunan, T. (2000). The role of rural small and medium industries and poverty alleviation in countries affected by the economic crisis: Indonesia, Philippines and Thailand. In Enhancement of competitiveness of small rural industries in a liberalized economic environment and the impact on poverty alleviation (pp. 2-31). New York: United Nations.

Tan, F.B. and Hunter, M.G. (2003). Using narrative inquiry in a study of information systems professionals. In Proceedings of the $36^{\text {th }}$ Hawaii International Conference on System Sciences (HICSS'03). Retrieved 20 April 2006, from http:// csdl2.computer/proceedings/hicss/2003/1874/08/187480253a.pdf.

Tashakkori, A. and Teddlie, C. (2003). Handbook of mixed methods in social \& behavioural research. Thousand Oaks, California: Sage.

Taylor, S.J. and Bogdan, R. (1984). Introduction to qualitative research methods: The search for meanings ( $2^{\text {nd }}$ ed.). New York: Wiley.

Thai Knowledge Centre (2007). ความรู้เรื่องภูมิปัญญาท้องถิ่น [Local wisdom]. Retrieved 16 May 2007, from http://www.tkc.go.th.

Thai Ruralnet (TRN). (2005). How villagers learn to solve the poverty problem: Poverty, self-reliance, self-economic, and Northeastern Mahachivarai. Burirum: Thai Ruralnet. 
Thorbjornsen, S. and Mouritsen, J. (2005). Individuals and collectivities in human capital statements. In N. Bukh, K.S. Christensen, and J. Mouritsen (Eds.), Knowledge management and intellectual capital: Establishing a field of practice (85-100). New York; Palgrave Macmillan.

Trade and Investment Division (TID). (2003). Implications of globalization for the development of agro-based industries in Developing countries of the ESCAP region: An overview. Retrieved 21 December 2004, from http:// unescap.org/publications/detail.asp?id=804

Triandis, H.C. (1995). Individualism \& collectivism. Boulder, Colorado: Westview Press.

Trice, H. and Beyer, J.M. (1993). The cultures of work organizations. Englewood Cliffs: Prentice-Hall.

Trinutchakorn, Y. (2002). Traditional medicine wisdom integration in community enterprises. In N. Petprasert and P. Wongkul (Eds.), Community enterprise: วิสาหกิจชุมชน: กลไกเศรษฐกิจฐานราก [Community enterprises: Economic mechanism for grass root] (pp. 161-183). Bangkok: Edison Press Production.

Truong, T. (2002). Gender and enterprise development in Vietnam under DOI-MOI: Issues for policy, research, and training. Retrieved 11 January 2005, from http://adlib.iss.nl/adlib/uploads/wp/wp363.pdf

Tyson, T. (1989). Working with groups. Hong Kong: The Macmillan Company of Australia PTY Ltd.

United Nation. (1995). FWCW platform for action women and the economy. Retrieved 3 March, from http://www.un.org/womenwatch/daw/beijing/platform/economy.htm

Van Den Bosch, F.A.J. and Van Wijk, R. (2001). Creation of managerial capabilities. In Sanchez, R. (Ed.), Knowledge management and organizational competence (pp. 159-176). New York: Oxford University Press.

Van Maanen, J. (1988). Tales of the field: On writing ethnography. Chicago: The University of Chicago Press.

Varela, F., Thompson, E., and Rosch, E. (1995), The embodied mind: Cognitive science and human experience. Massachusetts: The MIT Press.

Walaisatian, P. (1996). รายงานวิจัยฉบับสมบูรณ์ โครงการการสำรวจสถานะความรู้เกี่ยวกับธุรกิจชุมชน [The survey on community enterprise knowledge]. (Rep. No. RDG4/0001/2540). Bangkok, Thailand: Thammasart university, Department of Community Development, Faculty of Social Work.

Waldram, J.B. (1986). Traditional knowledge system: The recognition of indigenous history and science. Saskatchewan Indian Federate College Journal, 2(2), 115-124.

Walsh, J.P. and Ungson, G.R. (1991). Organisational memory. The Academy of Management Review, 16(1), 57-91.

Walsham, G. (1995). Interpretive case studies in IS research: nature and method. European Journal of Information Systems, 4, 74-81.

Wasuntiwongse, M. (1999). Micro and small enterprise development \& poverty alleviation in Thailand, working paper 5: The needs and characteristics of a sample of micro and small enterprises (MSEs) in Thailand. Retrieved 2 November 2004, from http://www.ilo.org/public/english/employment/ ent/papers/thai5.htm

Wathne, K., Roos, J. and Von Krogh, G. (1996). Toward a theory of knowledge transfer in a cooperative context. In G. Von Krogh and J. Roos (Eds.), 
Managing knowledge perspective on cooperation and competition (pp. 5581). Place of publication: Publisher

Wegner, D.M. (1987). Transactive memory: A contemporary analysis of the group mind. In B. Mullen and G.R. Goethals (eds.), Theories of group behaviour (pp. 185-208). New York: Springer-Verlag.

Weick, K.E. (1979). The social psychology of organization. Reading, MA: AddisonWesley Publishing.

Welsch, H.P. and Kuhns, B.A. (2005). Community-based enterprises: propositions and cases. Retrieved 10 January 2005, from http://www.usasbe.org/ knowledge/proceedings/2002/28.pdf

Wenger, E. (2004). Knowledge management as a doughnut: Shaping your knowledge strategy through communities of practice. Ivey Business Journal Online, 1.

Wenger, E., McDermott, R. and Snyder, W.M. (2002). A guide to managing knowledge cultivating communities of practice. Boston: Harvard Business School Publishing.

Wenger, E.C. and Snyder, W.M. (2000). Communities of practice: The organizational frontier. Harvard Business Review, 139-145.

White, S. (1999). Micro and small enterprise development \& poverty alleviation in Thailand, working paper 3: Creating an enabling environment for micro and small enterprise (MSE) development in Thailand. Retrieved 6 November 2004, from http://www.ilo.org/public/english/employment/ent/ papers/thai3.htm

WIEGO. (2005). The informal economy. Retrieved 17 January 2005, from http://www.wiego.org/main/fact1.shtml

Wiig, K.M. (1994). Knowledge management: The central management focus for intelligent-acting organizations. Arlington, TX: Schema Press.

Wiig, K.M. (1999). Introducing knowledge management into the enterprise. In J. Liebowitz (Ed.), Knowledge management handbook (pp. 3-41). Boca Raton; CRC Press.

Wijayaratna, C.M. (2000). Rural-community development in Asia and the Pacific- An overview. In Multi-country study mission on integrated local community development. Retrieved 23 December 2004, from http://www.apotokyo.org/icd/papers/E-Publications/02.IntegLocCommuDev/02-07.pdf

Williamson, K. (2006). Research in constructivist frameworks using ethnographic techniques. Library Trends, 55(1), 83-101.

Wong, K.Y. and Aspinwall, E. (2004). Characterizing knowledge management in the small business environment. Journal of Knowledge Management, 8(3), 4461.

Woodman, R.W., Sawyer, J.E. and Griffin, R.W. (1993). Toward a theory of organizational creativity. The Academy of Management Review, 18(2), 293321.

Wurman, R.S. (1997). Information architects. New York: Graphis.

Wyllie, J. (1998). Knowledge management tools - Ancient history or contemporary breakthrough? Retrieved 16 August 2005, from http://www.skyrme.com/updates/u16.htm

Yin, R.K. (1994). Case study research: Design and methods. Thousand Oaks, California: Sage.

Zack, M.H. (2002). Developing a knowledge strategy. In C.W. Choo and N. Bontis (Eds.), The strategic management of intellectual capital and organizational knowledge (pp. 255-276). New York: Oxford University Press. 
Zikmund, W.G. (1991). Business research methods ( $3^{\text {rd }}$ ed.). Chicago: The Dryden Press. 
Appendices

Appendix A

Human Ethic Approval Documents 


\section{Victoria \\ UNIVERSITY OF WELLINGTON \\ Te Whare Wānanga \\ o te Üpoko o te Ika a Māui

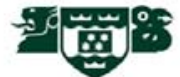

\section{INFORMATICS HUMAN ETHICS COMMITTEE}

\section{Application for Approval of Research Projects}

Please email applications to your supervisor, who will then email it to an

Informatics HEC member for a preliminary review.

Note: The Human Ethics Committee attempts to have all applications approved within 6 working days, but a longer period may be necessary if applications require substantial revision.

\section{NATURE OF PROPOSED RESEARCH:}

(a) Student Research

(b) If Student Research

Degree

$\mathrm{PhD}$

Course Code ...INFO690...

(c) Project Title: ...An Integrated Knowledge Management Model for Community

Enterprises: A case study of Rural Community Enterprise in Thailand

\section{INVESTIGATORS:}

(a) Principal Investigator

Name ...MS. Lanthom Jonjoubsong

e-mail address .lanthom.jonjoubsong@vuw.ac.nz

School/Dept/Group ...School of Information Management

(b) Other Researchers

Name

Position

(c) Supervisor (in the case of student research projects)

1) Prof. Gary Gorman

Principal Supervisor

2) Dr. Brian Harmer

Secondary Supervisor 


\title{
3 DURATION OF RESEARCH
} granted.

(a) Proposed starting date for data collection - After HEC approval has been

(Note: that NO part of the research requiring ethical approval may commence prior to approval being given) May 2006

(b)Proposed date of completion of project as a whole ...November 2007

\section{PROPOSED SOURCE/S OF FUNDING AND OTHER ETHICAL CONSIDERATIONS}

\author{
(a) Sources of funding for the project \\ Please indicate any ethical issues or conflicts of interest that may arise because of sources of \\ funding \\ e.g. restrictions on publication of results \\ (b) Is any professional code of ethics to be followed \\ $\mathbf{Y}(\mathbf{N}$ \\ If yes, name \\ (c) Is ethical approval required from any other body \\ If yes, name and indicate when/if approval will be given

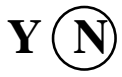

\section{DETAILS OF PROJECT}

Briefly Outline:

(a) The objectives of the project

To explore characteristics, organisational culture and capabilities of community enterprise in knowledge management (KM), and to propose a possible KM model

(b) Method of data collection
1) Document study
2) Narrative inquiry
3) Observation 
(c) The benefits and scientific value of the project

This research will contribute an analysis of KM model for community enterprises (CEs) in developing countries by investigating complex phenomena of organisational culture, operational systems and current knowledge management (KM) practice and capabilities of a CE through the case of rural CE in Thailand.

(d) Characteristics of the participants

1) Rural women who have low level of education.

2) NGO workers

(e) Method of recruitment

The participants will be recruited through the enterprise committee and advisor committee

- The enterprise committee members and advisor committee members will provide a list of participants to the researcher.

- $\quad$ The participants will be informed by the enterprise committee.

- The participants will have opportunities to talk with the researcher before the data collection taken place.

- The participants who are not willing to talk and uncomfortable with the researcher are free not to participate in the research without giving any reasons.

- The participants will have opportunity to ask questions before signing the consent form.

- The researcher will read out the consent form for the participants who cannot read and ask them for verbal consent with tape recording.

(f) Payments that are to be made/expenses to be reimbursed to participants No

(g) Other assistance (e.g. meals, transport) that is to be given to participants

1) Transportation expenses from villages to meeting places for in-group meeting and focus groups.

2) Meals for in-group meeting and focus groups.

(h) Any special hazards and/or inconvenience (including deception) that 
participants will encounter

None.

(i) State whether consent is for: (Please indicate as many as it applies)

(i) the collection of data

(ii) attribution of opinions or information

(iii) release of data to others

(iv) use for a conference report or a publication $\mathbf{Y}$ N

(v) use for some particular purpose (specify) $\mathbf{Y}(\mathrm{N}$

(j) How is informed consent to be obtained (see paragraphs 4.31(g), 5.2, 5.5 and 5.61 of the Guidelines)

(i) the research is strictly anonymous, an information sheet is supplied and informed consent is implied by voluntary participation in filling out a questionnaire for example (include a copy of the information sheet)

(ii) the research is not anonymous but is confidential and informed consent will be obtained through a signed consent form (include a copy of the consent form and information sheet) (Y) $\mathbf{N}$

(iii) the research is neither anonymous nor confidential and informed consent will be obtained through a signed consent form (include a copy of the consent form and information sheet)

$\mathbf{Y}(\mathbf{N})$

(iv) informed consent will be obtained by some other method (Rlease specify and provide details)

$\mathbf{Y}(\mathbf{N})$

With the exception of anonymous research as in (i), if it is proposed that written consent will not be obtained, please explain why

(k)If the research will not be conducted on a strictly anonymous basis state how issues of confidentiality of participants are to be ensured if this is intended. (See paragraph 4.3.1(e) of the Guidelines). (e.g. who will listen to tapes, see questionnaires or have access to data). Please ensure that you distinguish clearly between anonymity and confidentiality. Indicate which of these are applicable.

(i) access to the research data will be restricted to the investigator 
(ii) access to the research data will be restricted to the investigator and their supervisor (student research)

(iii) all opinions and data will be reported in aggregated form in such a way that individual persons or organisations are not identifiable

(iv) Other (please specify)

(Y) $\mathbf{N}$

Access to the research data will be restricted to research assistants if used and any research assistants will be required to sign confidently agreement.

(l) Procedure for the storage of, access to and disposal of data, both during and at the conclusion of the research. (see section 7 of the guidelines). Indicate which are applicable:

(i) all written material (questionnaires, interview notes, etc) will be kept

in a locked file and access is restricted to the investigator

(Y) $\mathrm{N}$

(ii) all electronic information will be kept in a password-protected file and access will be restricted to the investigator

(Y) $\mathbf{N}$

(iii) all questionnaires, interview notes and similar materials will be destroyed:

(a) at the conclusion of the research

or (b) _ _ _ years after the conclusion of the research

(iv) any audio or video recordings will be electronically wiped $\mathbf{N}$

(v) other procedures (please specify):

If data and material are not to be destroyed please indicate why and the procedures envisaged for ongoing storage and security

(m)Feedback procedures (See section 8 of the Guidelines). You should indicate whether feedback will be provided to participants and in what form. If feedback will not be given, indicate the reasons why.

The summary of the collected data will be sent to the enterprise which can be shared to each participant. 
(n)Reporting and publication of results. Please indicate which of the following are appropriate. The proposed form of publications should be indicated on the information sheet and/or consent form.

(i) publication in academic or professional journals

(ii) dissemination at academic or professional conferences

(Y) $\mathbf{N}$

$\mathbf{Y}$

(iii) deposit of the research paper or thesis in the University Library (student research)

(iv) a case study used for teaching purposes

(v) other (please specify)

Signature of investigators as listed on page 1 (including supervisors) and Chair of Informatics HEC.

NB: All investigators and the Chair of Informatics HEC must sign the form, then send it to Perumal Pillai for filing in the University's Research Office once the electronic application has been approved.

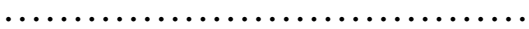

Date

Date

Date...

Supervisors:

Chair of Informatics HEC:

Date 


\section{APPLICATIONS FOR HUMAN ETHICS APPROVAL}

\section{CHECKLIST}

Have you read the Human Ethics Committee Policy?

Have you read the Faculty of Commerce and Administration's HEC Guide?

Is ethical approval required for your project?

Have you established whether informed consent needs to be obtained for your project?

In the case of student projects, have you consulted your supervisor about any human ethics implications of your research?

Have you included an information sheet for participants which explains the nature and purpose of your research, the proposed use of the material collected, who will have access to it, whether the data will be kept confidential to you, how anonymity or confidentiality is to be guaranteed?

Have you included a written consent form?

If not, have you explained on the application form why you do not need to get written consent?

Are you asking participants to give consent to:

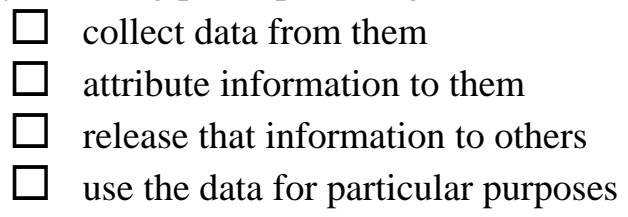

Have you indicated clearly to participants on the information sheet and/or consent form how they will be able to get feedback on the research from you (e.g. they may tick a box on the consent form indicating that they would like to be sent a summary), and how the data will be stored or disposed of at the conclusion of the research?

Have you included a copy of any questionnaire or interview checklist you propose using?

\section{POINTERS TO AVOID HAVING APPLICATIONS RETURNED BEFORE HEC REVIEW}

- The approval process is speeded up by not requiring the hard copy of your application form with the signatures on it at the initial review process. The complete application (HEC application form, info sheet, consent form, covering letter, questionnaire etc.) is to be emailed as an attachment in one file to your supervisor who will email it to an INFORMATICS HEC member for a preliminary review.

- Do not insert a date into item 3 a.

- Delete the "Y" or "N" option that is not required. DO NOT remove any other text from the application form.

- BOLD your answers if you wish but do not alter the font anywhere else in the form. 


\title{
Victoria \\ UNIVERSITY OF WELLINGTON \\ Te Whare Wänanga \\ o te Üpoko o te Ika a Māui

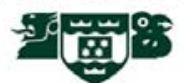

Participant Information Sheet for a study of

\section{An Integrated Knowledge Management Model for Community Enterprises:}

A case study of Rural Community Enterprise in Thailand

\author{
Researcher: Lanthom Jonjoubsong, School of Information Management \\ Victoria University of Wellington
}

I am a PhD student in Information Management at Victoria University of Wellington. As a part of this degree, I am undertaking a research project on integrated knowledge management model for community enterprises. This study is designed to explore characteristics, organisational culture, and current practice and capabilities of a community enterprise in knowledge management (KM) and to propose a possible $\mathrm{KM}$ model.

This study needs CE members, committee members, advisor and supporting agency members to participate. Participants will be asked to tell about their enterprise operational aspect, resources and current $\mathrm{KM}$ practice through narrative inquiry technique which I will not interrupt while participants are telling stories. The stories will be recorded through note taking and tap record, and participants' activities will be observed and recorded through note taking and photos with permission from participants.

Your name will not be identified in this report. The data collected will be seen only by my supervisors, Prof. Gary Gorman and Dr. Brian Harmer, myself and research assistants who will be required to sign confidently agreement. The summary of collected data will be sent to the enterprise. The thesis will be submitted for examination to the School of Information Management and deposited in the University library. In addition, the results will be sent to the research site and may be submitted to academic or professional journals and conferences as an article(s). The results will be used for other purposes only with your written consent. 
Participation is voluntary and the participants are free to withdraw from this project without giving reasons at any time before 31 July 2006. If they withdraw, all information that they have provided to date will be deleted from the study. This study is conducted under the Human Ethics Committee procedures of Victoria University of Wellington. Further information and questions about this project, please contact me at 64-4-3843008 or lanthom.jonjoubsong@vuw.ac.nz, and my supervisors, Prof. Gary Gorman and Dr. Brian Harmer at e-mail address gary.gorman@vuw.ac.nz and brian.harmer@vuw.ac.nz.

Regards

(Lanthom Jonjoubsong)

Researcher 


\title{
Victoria \\ UNIVERSITY OF WELLINGTON \\ Te Whare Wänanga \\ o te Üpoko o te Ika a Mäui

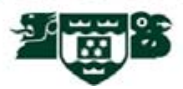

\section{Consent to Participation in Research \\ An Integrated Knowledge Management Model for Community Enterprises: A case study of Rural Community Enterprise in Thailand}

\begin{abstract}
I have been given and have understood an explanation of this research project. I have had an opportunity to ask questions and have them answered to my satisfaction. I understand that I may withdraw myself (or any information I have provided) from this project (before data collection and analysis is complete) without having to give reasons.
\end{abstract}

I understand that information or opinions which I have given will not be able to be identified me in any reports on this research.

I understand that the stories that I have told will be recorded.

I understand that my working processes may be monitored and all photos will be taken only under my permission.

I understand that the results of this research will be sent to the research site and examiners of the School of Information Management, and my name will not be referred to the report.

I understand that the data I provide will not be used for any other purpose or released to others without my written consent.

Signed:

Date:

Participant's name

Village's name 


\section{Victoria \\ UNIVERSITY OF WELLINGTON}

Te Whare Wānanga

o te Üpoko o te Ika a Māui

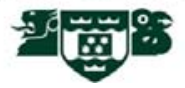

\section{รายละเอียดการศึกษาวิจัยเรื่อง โมเดลการจัดการข้อมูลแบบบูรณาการสำหรับธุรกิจชุมชน กรณีศึกษาธุรกิจชุมชนในประเทศไทย}

ข้าพเจ้า นางสาว ลั่นทม จอนจวบทรง เป็นนักศึกษาปริญญาเอกใน คณะการจัดการสารสนเทศ มหาวิทยาลัย วิคตอเรีย กรุงเวลลิงตัน ประเทศนิวซีแลนด์ กำลังศึกษาเรื่อง โมเดลการจัดการข้อมูลแบบบูรณาการสำหรับธุรกิจ ชุมชน สำหรับการศึกษาวิจัยในครั้งนี้ข้าพเจ้าได้เชิญสมาชิก คณะกรรมการบริหาร คณะกรรมที่ปรึกษา และ หน่วยงานที่สนับสนุน “แพรพรรณ” ธุรกิจชุมชนในภาคตะวันออกเฉียงเหนือ ของประเทศไทย เข้าร่วมใน การศึกษา โดยผู้เข้าร่วมจะเล่าเรื่องเกี่ยวกับการทำงานของแพรพรรณ และการจัดการองค์ความรู้ของหน่วยงาน ด้วยการเล่าเรื่อง (Narrative inquiry) เรื่องที่เล่าจะมีการจดบันทึกและบันทึกเทป กิจกรรมของผู้เข้าร่วมจะมีการ สังเกตการณ์จากนักวิจัย ทำการจดบันทึก และถ่ายภาพ โดยรับอนุญาติจากผู้เข้าร่วมก่อน

รายชื่อของผู้เข้าร่วมจะไม่สามารถตรวจสอบได้ในเอกสารงานวิจัย เอกสารและข้อมูลที่ท่านให้เพื่อการศึกษาใน ครั้งนี้จะใช้โดยข้าพเจ้า และอาจารย์ที่ปรึกษางานวิจัยนี้เท่านั้น ข้อมูลสรุปจะจัดส่งให้หน่วยงานของท่าน รายงาน การศึกษาจะจัดส่งให้คณะผู้สอบการวิจัย และจัดเก็บ ณ ห้องสมุดของมหาวิทยาลัย 9 ผลการศึกษาในครั้งนี้จะ จัดส่งให้กับ แพรพรรณ และ อาจนำไปเผยแพร่ในวารสาร และการประชุมทางวิชาการเท่านั่น หากข้าพเจ้า ต้องการนำไปเผยแพร่ในรูปแบบอื่นจะต้องขออนุญาตจากท่านเป็นลายลักษณ์อักษรเท่านั้น

การเข้าร่วมของท่านเป็นงานอาสาสมัคร ผู้เข้าร่วมสามารถขอถอนตัวจากโครงการนี้ได้ก่อนวันที่ 31 กรกฎาคม 2549 หากท่านถอนตัวจากโครงการนี้ ข้อมูลที่ท่านให้จะทำการลบออกจากรายงาน

งานวิจัยในครั้งนี้ดำเนินการภายใต้กระบวนการจริยาธรรมของมหาวิทยาลัยวิคตอเรีย กรุงเวลลิงตัน ประเทศ นิวซีแลนด์ หากท่านต้องการทราบข้อมูลเพิ่มเติมเกี่ยวกับโครงการวิจัยนี้ สามารถติดต่อข้าพเจ้าได้ที่เบอร์โทร 644-3843008 หรือ ที่อาจารย์ที่ปรึกษาของข้าพเจ้า ศจ.แกร์รี่ กอร์แมน และ ดร.ไบรอัน ฮาร์เมอร์ อีเมล์ gary.gorman@vuw.ac.nz หรือ brian.harmer@vuw.ac.nz

\section{ด้วยความนับถือ}

(นางสาว ลั่นทม จอนจวบทรง)

ผู้ศึกษาวิจัย 


\section{Victoria \\ UNIVERSITY OF WELLINGTON}

Te Whare Wänanga

o te Üpoko o te Ika a Māui

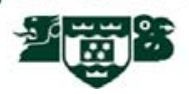

\section{หนังสือแสดงความยินยอมในการเข้าร่วมโครงการวิจัย \\ เรื่องโมเดลการจัดการข้อมูลแบบบูรณาการสำหรับธุรกิจชุมชน กรณีศึกษาธุรกิจชุมชนในประเทศ \\ ไทย}

ข้าพเจ้าได้รับการอธิบาย เกี่ยวกับโครงการวิจัยนี้เป็นที่เข้าใจ และได้มีโอกาสซักถาม พร้อมทั้งได้รับ คำตอบเป็นที่พอใจแล้ว ข้าพเจ้าเข้าใจเกี่ยวกับเข้าร่วมโครงการนี้ดีว่า ข้าพเจ้าสามารถขอถอนตัวจาก โครงการได้โดยไม่ต้องให้เหตุผลใด ๆ ก่อนที่การเก็บข้อจะสิ้นสุด และมีการวิเคราะห์ ข้าพเจ้า ยินยอมที่จะเข้าร่วมงานวิจัยดังนี้

\footnotetext{
$\square$ รับทราบว่ารายงานการวิจัยจะไม่สามารถตรวจสอบรายชื่อของข้าพเจ้าได้

$\square$ รับทราบว่าการให้ข้อมูลจะมีการบันทึกเทป

$\square$ รับทราบว่ากิจกรรมการทำงานของข้าพเจ้าจะมีการสังเกตการณ์และถ่ายภาพ
$\square$ รับทราบว่ารายงานจะจัดส่งให้แพรพรรณ และคณะผู้สอบงานวิจัยของทางคณะ การจัดการสารสนเทศ

$\square$ รับทราบว่าผลการวิจัยจะนำไปเผยแพร่ในวารสารหรือการประชุมทางวิชาการ หากผลงานวิจัยถูกนำไปเผยแพร่ทางอื่นต้องได้รับการอนุญาตเป็นลายลักษณ์ อักษรจากข้าพเจ้าก่อน
}

ลายมือชื่อ : วันที่:

ชื่อผู้เข้าร่วม

หมู่บ้าน 


\section{Appendix B}

\section{List of respondents' pseudonyms}

1. General Members

$\begin{array}{ll}\text { Ms. Malee } & \text { Nonthong group } \\ \text { Ms. Onanong } & \text { Nonthong group } \\ \text { Ms. Urai } & \text { Huafai group } \\ \text { Ms. Napaporn } & \text { Nongthum group } \\ \text { Ms. Lada } & \text { Suksomboon group } \\ \text { Ms. Malai } & \text { Suksomboon group } \\ \text { Ms. Suwannee } & \text { Suksomboon group } \\ \text { Ms. Mali } & \text { Laokwienhuk group }\end{array}$

2. Committee members

Ms. Walai

Fangnoi group

Ms. Kamolwan

Nonthong group

Ms. Raweewan

Huafai group

Ms. Prarichat

Huafai group

Ms. Pitsamai

Nongthum group

Ms. Kunya

Nongyanglan group

Ms. Sumaree

Nongyanglan group

3. Advisory committee member

Ms. Kannika

4. Office staff members

Ms. Penchun

Ms. Wijittra 


\section{Appendix C \\ An example of transcripts of the narratives in English}

\section{A transcript of committee member at Huafai village}

\section{How to be a member}

I became a member in the latter period because it was difficult with kids at the beginning. Members at this village chose me to be a committee member of Prae Pun because I'm available to travel in order to attend committee meetings and other activities. Most members of this village are seniors who don't like to go anywhere. For instance, grandma (my neighbour), she is busy with taking care of her grandson. In case of teenagers, they don't want to stay at the village and weaving. That is the same situation as when I was a teenager. I didn't want to be a member of Prae Pun. I wanted to work in Bangkok. I came back home when I got married. I came back to do farming and take care of my kids. After my kids grew up, I joined the group because I didn't want to be a worker transplanting and weeding on the sugarcane or tapioca fields.

I started weaving with place mats. The office set the quota of 60 pieces/month/member. Members may weave them up to 100-200 pieces/month, if we have orders. Then, we have woven blankets and scarves around 40-50 pieces/month/person. Now, we're having rug orders - 40 pieces/month/person. I used to weave big blankets with grandma because they need two people to weave at the same time.

Orders are distributed to the members according to expert areas and skills of each member. In addition, the members who are not good in those products won't accept the order. Right now, we have five to six members weaving place mats, five to six members weaving rugs, and some members weaving big blankets. Each blanket weaver has his/her own loom(s) which have specific designs. The office staff knows whom they will ask to weave when having orders. Nowadays, it's easy to distribute the orders because we have only around 20 members weaving. Some are busy at the moment, such as grandma is busy in taking care of her grandson. This village is good in dealing with order in both amount of order and deadline. We have woven plain 
cloth when we don't have any orders with the quota of 30 metres/month/person. For myself, I cannot reach the quotas because I've been busy with farm work.

At the beginning, there was no member weaving the place mat with "kit" because the senior members wove "mudmee" simple skirts. We weave kit by following old designs from old traditional pillows. Traditionally, we wove and produced kit pillows by ourselves for many years. We normally start weaving kit when we are 17-18 years old.

At the beginning, we were not Prae Pun's members. We asked Ban Nong Kham (exmember of Prae Pun) to sell our products. This village has been a member of Prae Pun since 1992. At the beginning, Prae Pun was not strong and had not much capital. We couldn't get pay when we sent our cloth to the office. We got the pay the next month, It was alright, because cotton was not expensive at that time. It costs around 40$50 \mathrm{baht} / \mathrm{kg}$. Currently, we get cash on the day that we send our cloth to the office.

\section{Weaving learning}

I started learning how to weave from my sister. She taught me instead of mom because our mom was died when I was 14-15 years old. I started weaving plain cloth as the material for a blouse. It was white cloth woven with a simple loom with 2-kao. Then, I learnt how to weave simple skirts, “pa kloa ma” (loincloth), and kit pillows. I learnt weaving once a year. I had very little time for learning because I spent lot of time cattle raising and playing. I learnt very fast. It was not hard for me to lean how to weave because I saw my parents weaving and helped them weaving. The hardest one is "kent fai" (cotton spinning). I cannot do it. I can do the other because I helped them doing in the evening. For dyeing, we used "mo nin", indigo and chemical dyeing.

I learnt natural dyeing from the staff of the handicraft centre. They told us the sorts of barks that can give colours. Then, we did experimentation by ourselves because the staff didn't know much about the trees in our areas. They weren't villagers at this village. We found that each bark can give more than one colour. If we add alum, it will give one colour. If we add lime, it will give another colour. Natural dyeing is harder than chemical dyeing. It requires boiling bark for long time in order to get 
colour, while chemical colour dyeing can be done immediately after boiling. We have experimentation together among three to four members. Each one brings any bark that is available to use. We do dyeing together when we have orders in order to get the same colours for each whole lot.

\section{Training}

I have not often attended training. I went to Ban Fang, Hua Thong, Nong Thum. They were workshops instead of training activities. We did the colours that we were good at.

I have been to Chiang Mai and Leoy. At the beginning, they went to the south of Thailand to study natural dyes. For Chiang Mai trip, we learnt about other handicraft projects, such as the Ban Sang handicraft village, in order to open our worldview. The Chiang Mai trip was organised for committee members with the objective of looking for alternative businesses in order to expand our business. We found that we could not do the same as them because our areas are not the same as their areas. It would be better to improve our weaving. They do bamboo crafts. We may exchange products for selling at our shop.

I was busy in taking care of my kids when they went for southern study trip. However, the participants of the trip trained us when they came back. They trained, for instance, 4-tarkor” weaving and “dok keaw” design. In addition, the participants who went to Roi-et trained us how to make kit with "tong leang” technique that keeps the kit designs on the kao for later weaving. Before using the tong leang technique, we had to prepare kit designs every time of weaving. So, study tours gave us additional knowledge.

Recently, the government has set up many weaving group in this areas. They invited us to teach how to make big blankets. They gave us $300 \mathrm{baht} /$ day. We taught them everything from thread preparing (kon dai) to weaving. They can weave but their cloth does not sell well. Now, they are members of OTOP. Now, we teach only members in our village. They call on us to give advice at their homes when they cannot do it. Also, we help them design cloth when they do thread preparing. We take 
about a day in helping them design plain cloth. In addition, we help them fix any problems, such as wrong patterns of kit and blinding thread. We do not charge them because we have to help each other in the group.

I learnt kit weaving from my sister. They taught me because I had nothing to do at that time. Nowadays, I cannot do it because my eyesight is not good. I have to ask some people to prepare kit pattern (keab kao) for me, then, I can weave kit.

\section{Committee membership}

We have to check cloth and send to the office once a month. Then, we charge $2 \%$ from members for shipping cost and group activities.

For cloth checking, plain cloth is checked for texture, then the committee records amount of cloth of each member. We have to check all cloths and take out cloth that does not meet the standard. This can help the office staff in cloth checking. We know what kinds of cloths are good because we have woven for long time. In addition, the office staff also tells us our mistake, we have to record that mistake and talk among the members in the village.

The committee members have to attend regular meetings. We have to share our village situations and problems with the committee. Then, the committee will discuss and find out solutions. After that, we have a village meeting to share information from the committee meeting. For instance, the committee decided to stop blanket weaving in the low selling period. We had to tell members in the village to stop weaving until we had more orders. The recent example is we had a meeting yesterday at the village in order to inform the members who are credit union members to attend the annual meeting.

\section{Cloth selling}

I often join office staff members for handicraft fairs, such as the Thai craft fair. We need three to four people for the Thai craft fair. We have to demonstrate cotton spinning and weaving next month. Thai craft invited us to attend training on pattern design in Kumpangsean district (Nakornprathum province). They invited a Philippino 
resource person to tell us about cotton product design, such as curtains, bags and blouses.

\section{Price setting}

Prices are set by the office staff. They have to calculate based on cotton cost and labour cost which is calculated at the lowest rate of 60 baht/day. We have not got much income. The committee members have no responsibility for price setting. We have the role of defending to increase the prices at committee meetings. Normally, the prices are increased once a year but we have not increased the prices for many years because we have a lot of competitors. For instance, this village has many weaving groups that use chemical dye.

\section{Cloth design}

We can design the cloth by ourselves for monthly weaving. We have to weave as ordered when we have specific orders. We can mix coloured threads in our cloths as we like, so each member will make different designs. We have to design before preparing thread (khon dai).

\section{Kit design}

Normally, we use previous designs. We just change colours. However, we have designed new designs, such as dinosaur and forest. We have to draw on paper, then, we have to try weaving. If it is not good, we will take it out. Currently, we have assigned Ban Nongyanglan to weave kit, so we do not weave the same products. However, we asked them for an agreement in order to ensure their abilities in weaving such products.

\section{“Mo Nin” dyeing}

I cannot do "Mo Nin" dyeing. It was mobile commercial dyeing. They did for us at home. I'm not sure whether it is “mo hom” or chemical dyes, but it wasn't indigo. Mo Nin is black colour, meanwhile mo hom is dark blue. There is no hom tree in this area. It is planted in the North of Isan. Mo Nin colour has long life and a nice smell. Currently, there has been no Mo Nin in the village for over 10 years. 


\section{Colour dyeing workshop at Ban Nongyanlan}

We make green colour with small mango bark. Really, green colour can be made with any leaves by double dyeing with "makleo". This is because the first dyeing gives a yellow colour, then we have to redye with makleo in order to get green colour. Also, grandma who is my neighbour she makes red colour with mangwan mango, redyed with makleo. Any green leaves, such as "yo" and "kun”, can give green colour. We did this colour because we have makleo and manwan mango at our forest. Mangwan mango can be found in the paddy field. We are also good at brown colour with " $D u$ " and rusty water or lime water. Other villages have difficulty in finding these trees. For example, Nong Thum does not have a small forest at their paddy fields.

At the beginning, we didn't know that small mango bark could give dark green colour. They told us only small mango bark can make green colour, but we needed dark green. Then, we tried with double dyeing technique by using makleo. So, we got the dark green as we want. If we want light green, we will not redye with makleo. In fact, we heard that makleo can make dark colour from members. We always tell each other when we know any techniques. Generally, makleo is used to make black colour for silk. At beginning, there were no villages dyeing with this technique. Many members asked about this technique. Both green makleo and ripened makleo can be used. We have to ferment makleo and collect only the liquid. We have seen our seniors using makleo for fishnet dyeing. We can collect makleo only once a year in this season. So, we have planned to collect and ferment them for using whole year. Natural dyeing depends on seasons, such as bark dyeing is hard to do in the rainy season because bark absorbs a lot of water, but fruit dyeing is good to do in the rainy season. If we don't have makleo, we will use mud. Previously, we soaked cloth the buffalo mire over night. Now, we don't have a buffalo mire. We have to take mud from the field to do it at home and can soak only a few minutes. Most farmers have sold their buffalos and bought cows. My neighbour just did this technique when she made red colour. Other villages also use this technique. Mud can help to make darker colours. Sawai just did samol dyeing with mud last month.

\section{Bark selecting}

We have to hew bark in order to check a colour. It gives the colour the same as oil wood colour. For instance, it will give red colour, if its oil wood is red colour. 
mangwan mango can give red colour, but young mangwan mango trees would give light colour, so we have to add copper sulfate in order to make dark colour. Red colour from mangwan mango is different from red colour of sticklac which gives pink-red. However sticklac can give different colour for cotton and silk dyeing. Cotton dyeing with sticklac is harder than silk dyeing. Our village can't does sticklac dyeing. We weren't train to do sticklac dyeing. But I practice sticklac dyeing with "Mae" Oun and "Mae” Som for my own consumption weaving.

\section{Big blanket weaving}

I used to weave big blankets with grandma but now she is busy with her grandson. So, I have to weave them with another grandma. She used to weave it with her daughter, but now her daughter has gone to work. We can do it well together, whereas some members had to practise for long time in order to weave together, because they couldn't pull a reed at the same time.

\section{Designs of big blankets}

There are three designs - "Nuay”, “jug jig”, and “Bug hyay”. Each member has one loom for big blanket weaving. Normally, big blankets are used as bed cover sheets. "Mae” Nong weaves Nuay design, another member weaves bug hyay design, and I weave jug jig design. Other members weave common blankets using the three designs. We weave any design, when we have many orders. This is because we can weave any designs that have looms prepared. However the jug jig is the hardest one, because it is hard to prepare the loom (kleab kao). Even Mae Bong can't weave jug jig design. I have looms for every design.

\section{Kleab Kao}

We have not enough time to prepare it. We ask people in Roi-et province to make it, but they can't do jug jig design. They told us that they could, but it wasn't the jug jig design. So, we have to do it by ourselves. At the beginning, grandma saw the jug jig design when she went for study tour. She drew the design and prepared the loom by herself. Jug jig is hard to weave. Some members can weave it but it isn't stretched. We have only three to four members who can weave good jug jig design. Bug hyey design is the simplest one. Nuay is hard to prepare the loom as well. Blanket weaving is hard. We have to carefully check the design. We have taught the members in this 
village to weave and prepare looms. But they like to ask people in Roi-et to prepare for them. The narrow reed is 250 baht and the wide loom is 350 baht. Normally, I used to prepare the loom for them, but now my eyesight is not good. I don't prepare the kit design either. I ask someone to do it. It costs 10 baht/line of design. I used to teach the villagers in prepare kit design with tong leang technique, but now I can help them to fix the problem of kit weaving.

\section{Rug weaving}

Rugs have the same designs as blankets but they are smaller and need specific warp preparing in order to make float patterns. Most members don't want to weave rugs, because they are hard work and use a lot of thread. They can weave only seven to eight rugs/day and get only 80 baht/piece while the cotton cost 70 baht/piece. Currently, cotton is expensive - 80-90 baht/kg, whereas it used to be $50 \mathrm{baht} / \mathrm{kg}$.

We started rug weaving because the office ordered. We make them with trial and error. At the beginning, they were no patterns on rugs, because we used metal reeds. The patterns come up only by using wooden reeds. We found this technique, because only grandma's rugs had patterns. She was only one member that used wooden reed. Wooden reed is cheaper and light but it's difficult to find it.

These village members mainly weave big blankets because other villages don't want to do. We used to weave place mats but, currently, Ban Nongyanlan's members do. We have skills at blanket weaving because we used to weave blankets for our own consumption but they are simple pattern weaving with 2-kao. Generally, Isan weave blankets for themselves but they are narrow-one metre, but our blankets are wide: $1.2,1.3$ or 1.5 metres. 


\section{Appendix D}

\section{Data analyses documents}

1. Data classification diagrams

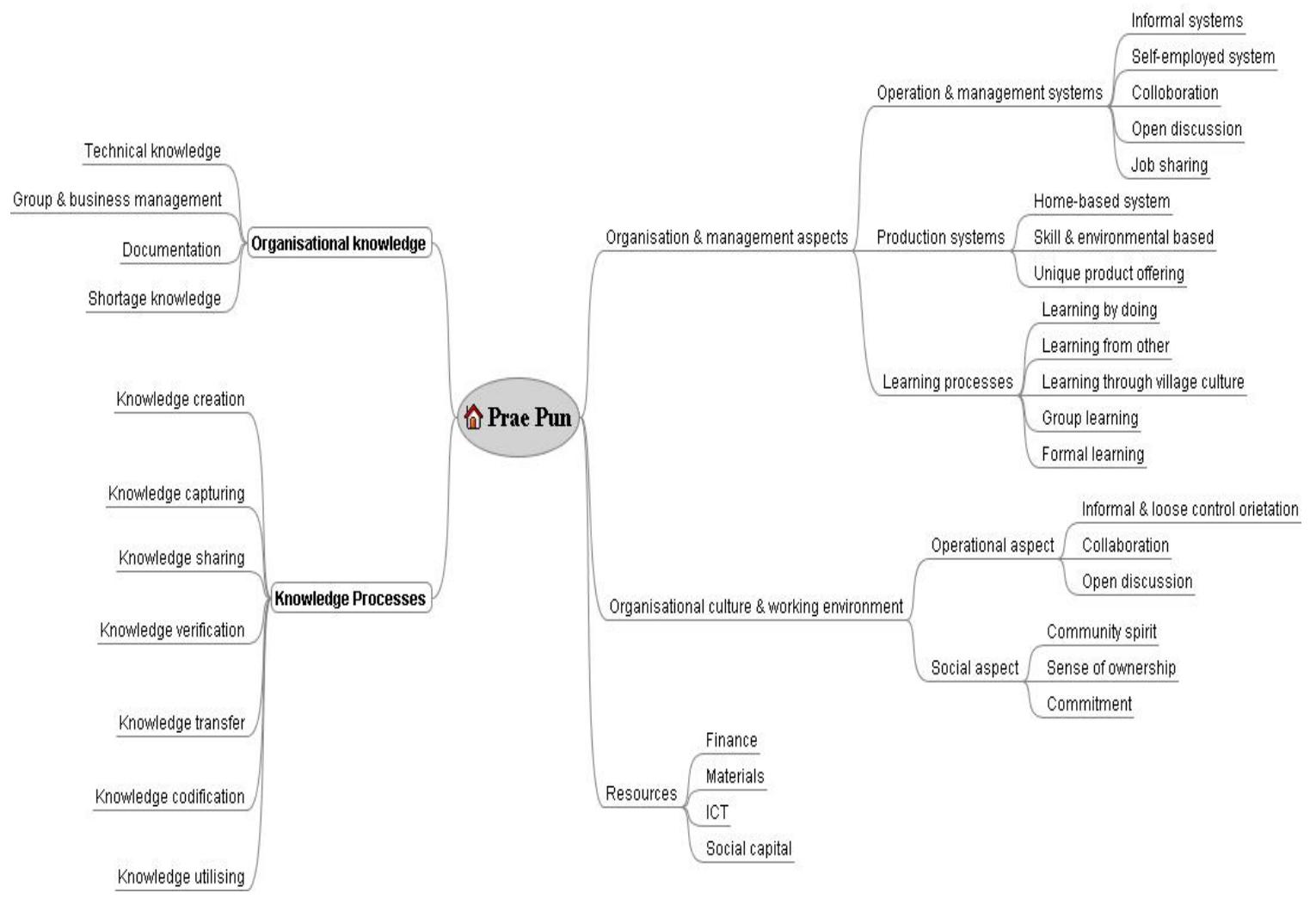

Figure D1 A classification for data analysis 


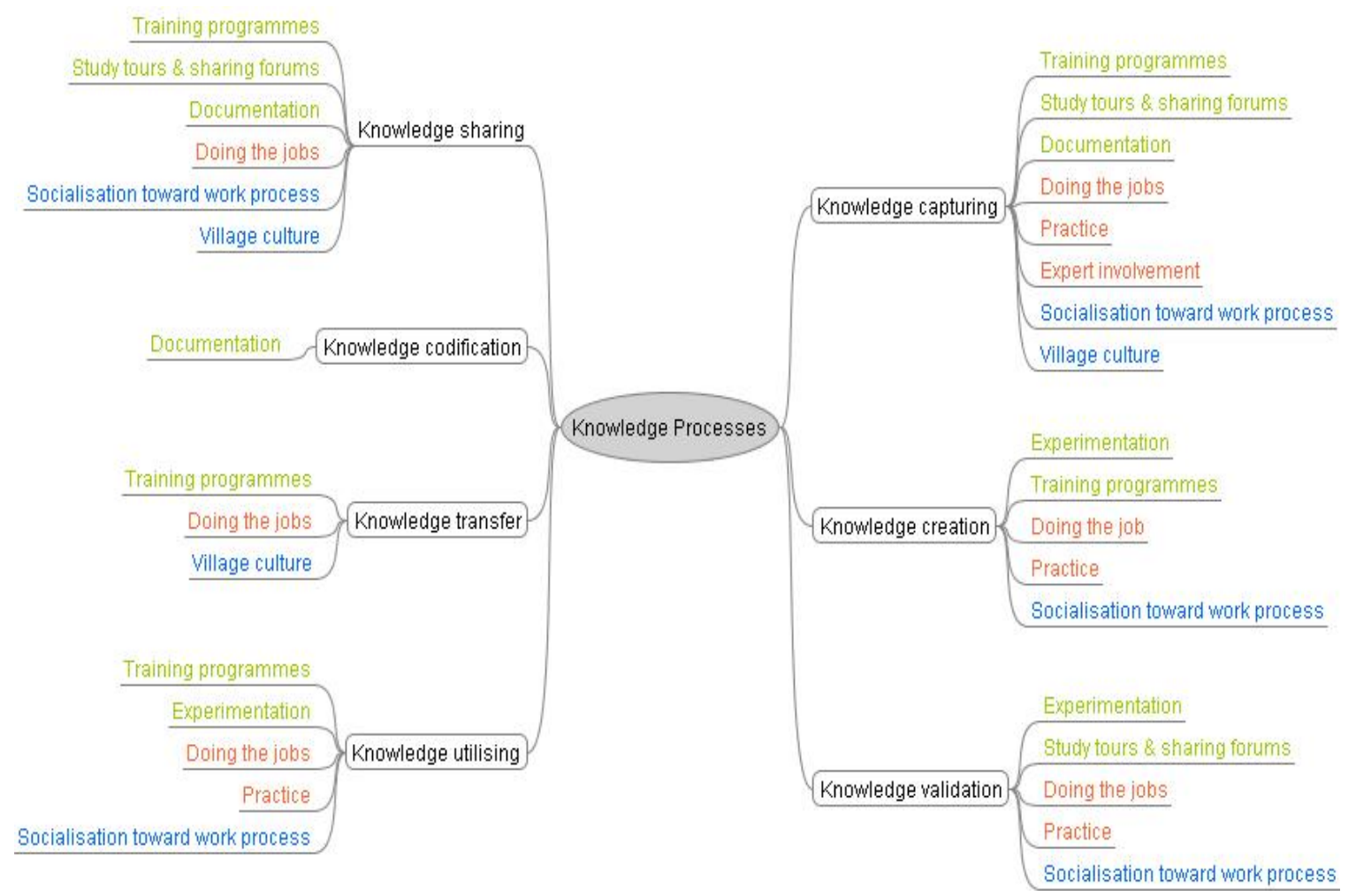

Remark: Green letters represent educational activities, Red letters represent practical activities and blue letters represent socialization

Figure D2 Codes for data classification of knowledge processes 


\section{An example of data classification in English}

Table E1 An example of data coding of the narratives of Mrs. Raweewan in the category of organizational knowledge

\begin{tabular}{|c|c|c|}
\hline $\begin{array}{l}\text { Text } \\
\text { No. }\end{array}$ & Text & Category \\
\hline 4 & $\begin{array}{l}\text { I started weaving with place mats. The office set the } \\
\text { quota of } 60 \text { pieces/month/member. The members } \\
\text { may weave up to } 100-200 \text { pieces/month, if we have } \\
\text { orders. Then, we have woven blankets and scarves, } \\
\text { around } 40-50 \text { pieces/month/person. Now, we're } \\
\text { having rug orders - } 40 \text { pieces/month/person. }\end{array}$ & knowledge-business \\
\hline 14 & $\begin{array}{l}\text { At the beginning, our group was not strong and had } \\
\text { not much capital. We couldn't get a pay when we } \\
\text { sent our cloth to the office. We got a pay in next } \\
\text { month. Currently, we can get cash on the day that } \\
\text { we send our cloth to the office. }\end{array}$ & knowledge-business \\
\hline 24 & $\begin{array}{l}\text { We found that we could not do the same as them } \\
\text { because our areas are not the same as their areas. It } \\
\text { would be better to improve our weaving. They do } \\
\text { bamboo crafts. We may exchange products for } \\
\text { selling at our shop. }\end{array}$ & knowledge-business \\
\hline 29 & $\begin{array}{l}\text { the government has set up many weaving groups in } \\
\text { this areas. They invited us to teach how to make a } \\
\text { big blanket. They gave us } 300 \text { baht/day. We taught } \\
\text { them everything from thread preparing (kon dai) to } \\
\text { weaving. They can weave but their cloth does not } \\
\text { sell well. }\end{array}$ & Knowledge-business \\
\hline 43 & $\begin{array}{l}\text { Prices are set by the office staff. They have to } \\
\text { calculate based on cotton cost and plus labour cost } \\
\text { which is calculated at lowest rate of } 60 \text { baht/day. } \\
\text { We have not got much income. }\end{array}$ & Knowledge-business \\
\hline 45 & $\begin{array}{l}\text { Normally, the prices are increased once a year but } \\
\text { we had not increased the prices for many years } \\
\text { because we have a lot of competitors. For instance, } \\
\text { this village has many weaving groups that use } \\
\text { chemical dye. }\end{array}$ & knowledge-business \\
\hline 35 & $\begin{array}{l}\text { We have to check cloth and send to the office once } \\
\text { a month. Then, we charge } 2 \% \text { from members for } \\
\text { shipping cost and group activities. }\end{array}$ & Knowledge-business \\
\hline 36 & $\begin{array}{l}\text { For cloth checking, plain cloth will be checked } \\
\text { texture, then the committee records amount of cloth } \\
\text { of each member. }\end{array}$ & $\begin{array}{l}\text { knowledge- } \\
\text { documentation }\end{array}$ \\
\hline
\end{tabular}


(Cont.)

\begin{tabular}{|c|c|c|}
\hline $\begin{array}{l}\text { Text } \\
\text { No. }\end{array}$ & Text & Category \\
\hline 38 & $\begin{array}{l}\text { We know what kinds of cloths are good because we } \\
\text { have woven for long time. In addition, the office } \\
\text { staff also tells us our mistake, we have to record } \\
\text { that mistake and talk among the members in the } \\
\text { village. }\end{array}$ & $\begin{array}{l}\text { knowledge- } \\
\text { documentation }\end{array}$ \\
\hline 17 & $\begin{array}{l}\text { The hardest one is “Kent Fai” (cotton spinning). I } \\
\text { cannot do it. }\end{array}$ & knowledge-shortage \\
\hline 42 & $\begin{array}{l}\text { Thai craft invited us to attend training on pattern } \\
\text { design in Kumpangsean district (Nakornprathum } \\
\text { province). They invited a Philippino resource } \\
\text { person to tell us about cotton product design, such } \\
\text { as curtains, bags and blouses. }\end{array}$ & knowledge-shortage \\
\hline 45 & $\begin{array}{l}\text { Normally, the prices are increased once a year but } \\
\text { we had not increased the prices for many years } \\
\text { because we have a lot of competitors. For instance, } \\
\text { this village has many weaving groups that use } \\
\text { chemical dye. }\end{array}$ & knowledge-shortage \\
\hline 62 & $\begin{array}{l}\text { However the jug jig is the hardest one, because it is } \\
\text { hard to prepare loom (kleab kao). Even Mae Bong } \\
\text { can't weave jug jig design. I have looms for every } \\
\text { design... jug jig is hard to weave. Some members } \\
\text { can weave it but it isn't stretched. We have only 3-4 } \\
\text { members who can weave good jug jig design. }\end{array}$ & knowledge-shortage \\
\hline 8 & $\begin{array}{l}\text { Each blanket weaver has her/his own loom(s) which } \\
\text { have specific designs. The office staff knows whom } \\
\text { they will ask to weave when having orders. }\end{array}$ & knowledge-technical \\
\hline 24 & $\begin{array}{l}\text { We found that we could not do as them because our } \\
\text { areas are not the same as their areas. It would be } \\
\text { better to improve our weaving. They do bamboo } \\
\text { crafts. We may exchange products for selling at our } \\
\text { shop. }\end{array}$ & knowledge-technical \\
\hline 29 & $\begin{array}{l}\text { the government has set up many weaving groups in } \\
\text { this areas. They invited us to teach how to make a } \\
\text { big blanket. They gave us } 300 \text { baht/day. We taught } \\
\text { them everything from thread preparing (kon dai) to } \\
\text { weaving. They can weave but their cloth does not } \\
\text { sell well. }\end{array}$ & knowledge-technical \\
\hline 45 & $\begin{array}{l}\text { Normally, the prices are increased once a year but } \\
\text { we had not increased the prices for many years } \\
\text { because we have a lot of competitors. For instance, } \\
\text { this village has many weaving groups that use } \\
\text { chemical dye. }\end{array}$ & knowledge-technical \\
\hline
\end{tabular}


(Cont.)

\begin{tabular}{|c|c|c|}
\hline $\begin{array}{l}\text { Text } \\
\text { No. }\end{array}$ & Text & Category \\
\hline 47 & $\begin{array}{l}\text { Normally, we use previous designs. We just change } \\
\text { colours. However, we have designed new designs, } \\
\text { such as dinosaur and forest. We have to draw on } \\
\text { paper, then we have to try weaving. If it isn't good, } \\
\text { we will take it out. }\end{array}$ & knowledge-technical \\
\hline 50 & $\begin{array}{l}\text { At the beginning, we didn't know that small mango } \\
\text { bark can give dark green colour. They told us only } \\
\text { small mango bark can make green colour, but we } \\
\text { needed dark green. Then, we tried with double } \\
\text { dyeing technique by using makleo. So, we got the } \\
\text { dark green as we want. In fact, we heard about } \\
\text { makleo can make dark colour from members. We } \\
\text { always tell each other when we know any } \\
\text { techniques. }\end{array}$ & knowledge-technical \\
\hline 52 & $\begin{array}{l}\text { We have seen our seniors using makleo for fishnet } \\
\text { dyeing. We can collect makleo only once a year in } \\
\text { this season. So, we have planned to collect and } \\
\text { ferment them for using whole year. }\end{array}$ & knowledge-technical \\
\hline 53 & $\begin{array}{l}\text { Natural dyeing depends on seasons, such as bark } \\
\text { dyeing is hard to do in rainy season because bark } \\
\text { absorbs a lot of water, but fruit dyeing is good to do } \\
\text { in rainy season. }\end{array}$ & knowledge-technical \\
\hline 54 & $\begin{array}{l}\text { Previously, we soaked cloth at the buffalo mire over } \\
\text { night. Now, we don't have a buffalo mire. We have } \\
\text { to take mud from the field to do it at home and can } \\
\text { soak only a few minutes. }\end{array}$ & knowledge-technical \\
\hline 55 & $\begin{array}{l}\text { My neighbour just did this technique when she } \\
\text { made red colour. Other villages also use this } \\
\text { technique. Mud can help to make darker colours. } \\
\text { Sawai just did Samol dyeing with mud last month. }\end{array}$ & knowledge-technical \\
\hline 57 & $\begin{array}{l}\text { Mangwan mango can give red colour, but young } \\
\text { Mangwan mango tree would give light colour, so } \\
\text { we have to add copper sulfate in order to make dark } \\
\text { colour. Red colour from Mangwan mango is } \\
\text { different from red colour of sticklac which gives } \\
\text { pink-red. }\end{array}$ & knowledge-technical \\
\hline 60 & $\begin{array}{l}\text { We can do well together, whereas some members } \\
\text { have to practise for long time in order to weave } \\
\text { together, because they couldn’t pull a reed at the } \\
\text { same time. }\end{array}$ & knowledge-technical \\
\hline 61 & $\begin{array}{l}\text { There are three designs - 'Nuay’, 'jug jig’, and } \\
\text { 'Bug hyay'. Each member has one loom for big } \\
\text { blanket weaving... 'Mae’ Nong weaves nuay design, } \\
\text { another member weaves Bug hyay design, and I } \\
\text { weave jug jig design. Other members weave } \\
\text { common blankets with using the three designs. }\end{array}$ & knowledge-technical \\
\hline
\end{tabular}


(Cont.)

\begin{tabular}{|c|c|c|}
\hline $\begin{array}{l}\text { Text } \\
\text { No. }\end{array}$ & Text & Category \\
\hline 62 & $\begin{array}{l}\text { However the jug jig is the hardest one, because it is } \\
\text { hard to prepare loom (kleab kao). Even Mae Bong } \\
\text { can't weave jug jig design. I have looms for every } \\
\text { design... jug jig is hard to weave. Some members } \\
\text { can weave it but it isn't stretched. We have only 3-4 } \\
\text { members who can weave good jug jig design. }\end{array}$ & knowledge-technical \\
\hline 63 & $\begin{array}{l}\text { We ask people in Roi-et province to make it, but } \\
\text { they can't do jug jig design. They told us that they } \\
\text { can, but it wasn't the jug jig design. So, we have to } \\
\text { do it by ourselves. }\end{array}$ & knowledge-technical \\
\hline 67 & $\begin{array}{l}\text { I used to teach the villagers in prepare Kit design } \\
\text { with "Thong leang” technique, but now I can help } \\
\text { them to fix the problem of kit weaving. }\end{array}$ & knowledge-technical \\
\hline 71 & $\begin{array}{l}\text { These village members mainly weave big blankets } \\
\text { because other villages don’t want to do. We used to } \\
\text { weave place mats but, currently, Ban Nongyanlan’s } \\
\text { members do. We have skills in blanket weaving } \\
\text { because we used to weave blankets for our own } \\
\text { consumption but they were simple pattern woven } \\
\text { with “2-Kao”. }\end{array}$ & knowledge-technical \\
\hline 4 & $\begin{array}{l}\text { I started weaving with place mats. The office set the } \\
\text { quota of } 60 \text { pieces/month/member. The members } \\
\text { may weave up to } 100-200 \text { pieces/month, if we have } \\
\text { orders. Then, we have woven blankets and scarves } \\
\text { around } 40-50 \text { pieces/month/person. Now, we're } \\
\text { having rug orders - } 40 \text { pieces/month/person. }\end{array}$ & $\begin{array}{l}\text { Knowledge- } \\
\text { Technical/cloth }\end{array}$ \\
\hline 46 & $\begin{array}{l}\text { We can design the cloth by ourselves for monthly } \\
\text { weaving. We have to weave as orders when we } \\
\text { have specific orders. We can mix coloured threads } \\
\text { in our cloths as we like, so each member will make } \\
\text { different designs. }\end{array}$ & $\begin{array}{l}\text { Knowledge- } \\
\text { technical/cloth }\end{array}$ \\
\hline 47 & $\begin{array}{l}\text { Normally, we use previous designs. We just change } \\
\text { colours. However, we have designed new designs, } \\
\text { such as dinosaur and forest. We have to draw on } \\
\text { paper, then, we have to try weaving. If it isn't good, } \\
\text { we will take it out. }\end{array}$ & $\begin{array}{l}\text { Knowledge- } \\
\text { technical/cloth }\end{array}$ \\
\hline 68 & $\begin{array}{l}\text { Rug has designs the same as blanket but it is smaller } \\
\text { and needs specific warp preparing in order to make } \\
\text { float pattern. }\end{array}$ & $\begin{array}{l}\text { Knowledge- } \\
\text { technical/cloth }\end{array}$ \\
\hline
\end{tabular}


(Cont.)

\begin{tabular}{|c|c|c|}
\hline $\begin{array}{l}\text { Text } \\
\text { No. }\end{array}$ & Text & Category \\
\hline 70 & $\begin{array}{l}\text { We started rug weaving because the office ordered. } \\
\text { We did it with trial and error. At the beginning, it } \\
\text { didn’t have patterns on rug, because we used metal } \\
\text { reeds. It would give a pattern when using wooden } \\
\text { reed. We found this technique, because only } \\
\text { grandma's rugs had a pattern. She was only one } \\
\text { member that used wooden reed. }\end{array}$ & $\begin{array}{l}\text { Knowledge- } \\
\text { technical/cloth }\end{array}$ \\
\hline 12 & $\begin{array}{l}\text { At the beginning, there was no member weaving the } \\
\text { place mat with kit because the senior members } \\
\text { weave "Mudmee" simple skirt }\end{array}$ & knowledge-tecnical \\
\hline 70 & $\begin{array}{l}\text { We started rug weaving because the office ordered. } \\
\text { We did it with trial and error. At the beginning, it } \\
\text { didn't have patterns on rug, because we used metal } \\
\text { reeds. It would give a pattern when using wooden } \\
\text { reed. We found this technique, because only } \\
\text { grandma's rugs had a pattern. She was only one } \\
\text { member that used wooden reed. }\end{array}$ & knowledge-technical \\
\hline 1 & $\begin{array}{l}\text { I just became a member in the latter period because } \\
\text { it was difficult with kids at the beginning... When I } \\
\text { was a teenager, I didn't want to be a member of } \\
\text { Prae Pun. I wanted to work in Bangkok. I came } \\
\text { back home when I got marry. I came back to do } \\
\text { farming and taking care my kids. }\end{array}$ & $\begin{array}{l}\text { Member - } \\
\text { knowledge/external }\end{array}$ \\
\hline 4 & $\begin{array}{l}\text { I started weaving with place mats. The office set the } \\
\text { quota of } 60 \text { pieces/month/member. The members } \\
\text { may weave up to } 100-200 \text { pieces/month, if we have } \\
\text { orders. Then, we have woven blankets and scarves } \\
\text { around } 40-50 \text { pieces/month/person. Now, we're } \\
\text { having rug orders - } 40 \text { pieces/month/person. }\end{array}$ & $\begin{array}{l}\text { Member - } \\
\text { knowledge/tech }\end{array}$ \\
\hline 6 & $\begin{array}{l}\text { Orders are distributed to the members according to } \\
\text { expert areas and skills of each member. In addition, } \\
\text { the members who are not good in those products } \\
\text { won't accept the order. }\end{array}$ & $\begin{array}{l}\text { Member - } \\
\text { knowledge/tech }\end{array}$ \\
\hline 7 & $\begin{array}{l}\text { Right now, we have 5-6 members weaving place } \\
\text { mats, 5-6 members weaving rugs, and some } \\
\text { members weaving big blankets. }\end{array}$ & $\begin{array}{l}\text { Member - } \\
\text { knowledge/tech }\end{array}$ \\
\hline 8 & $\begin{array}{l}\text { Each blanket weaver has her/his own loom(s) which } \\
\text { have specific designs. The office staff knows whom } \\
\text { they will ask to weave when having orders. }\end{array}$ & $\begin{array}{l}\text { Member - } \\
\text { knowledge/tech }\end{array}$ \\
\hline 10 & $\begin{array}{l}\text { This village is good in dealing with orders in both } \\
\text { volume and deadlines. }\end{array}$ & $\begin{array}{l}\text { Member - } \\
\text { knowledge/tech }\end{array}$ \\
\hline
\end{tabular}


(Cont.)

\begin{tabular}{|c|l|l|}
\hline $\begin{array}{c}\text { Text } \\
\text { No. }\end{array}$ & \multicolumn{1}{|c|}{ Text } & Category \\
\hline 13 & $\begin{array}{l}\text { We weave kit by following old designs from old } \\
\text { traditional pillows. Traditionally, we wove and } \\
\text { produced kit pillows by ourselves for many years. } \\
\text { We normally start weaving kit when we are 17-18 } \\
\text { years old. }\end{array}$ & $\begin{array}{l}\text { Member - } \\
\text { knowledge/tech }\end{array}$ \\
\hline 19 & $\begin{array}{l}\text { I learnt natural dyes from the staff of the handicraft } \\
\text { centre. They told us sorts of barks that can give } \\
\text { colours. Then, we did experiments by ourselves } \\
\text { because the staff didn't know much about trees in } \\
\text { our areas. We found that each bark can give more } \\
\text { than one colour. If we add alum, it will give one } \\
\text { colour. If we add lime, it will give another colour. }\end{array}$ & $\begin{array}{l}\text { Member - } \\
\text { knowledge/tech }\end{array}$ \\
\hline
\end{tabular}


Table E2 A example of data coding of the narratives of Mrs. Raweewan in the category of knowledge processes

\begin{tabular}{|c|c|c|}
\hline $\begin{array}{l}\text { Text } \\
\text { No. }\end{array}$ & Text & Category \\
\hline 50 & $\begin{array}{l}\text { At the beginning, we didn't know that small mango } \\
\text { bark can give dark green colour. They told us only } \\
\text { small mango bark can make green colour, but we } \\
\text { needed dark green. Then, we tried with double dyeing } \\
\text { technique by using makleo. So, we got the dark green } \\
\text { as we want. In fact, we heard about makleo can make } \\
\text { dark colour from members. We always tell each other } \\
\text { when we know any techniques. }\end{array}$ & $\begin{array}{l}\text { K.capture - } \\
\text { experiment }\end{array}$ \\
\hline 22 & $\begin{array}{l}\text { I’ve not often attended training. I went to Ban Fang, } \\
\text { Hua Thong, Nong Thum. They were workshops instead } \\
\text { of training. We did the colours that we were good at. }\end{array}$ & K.capture - training \\
\hline 42 & $\begin{array}{l}\text { Thai craft invited us to attend training on pattern design } \\
\text { in Kumpangsean district (Nakornprathum province). } \\
\text { They invited a Philippino resource person to tell us } \\
\text { about cotton product design, such as curtains, bags and } \\
\text { blouses. }\end{array}$ & K.capture - training \\
\hline 27 & $\begin{array}{l}\text { the participants who went to Roi-et trained us how to } \\
\text { make kit with "Thong Leang” technique that keeps Kit } \\
\text { designs at loom (“Kao") for later weaving. Before } \\
\text { using Thong Leang technique, we had to prepare Kit } \\
\text { designs every time of weaving. }\end{array}$ & K.capture - training \\
\hline 13 & $\begin{array}{l}\text { We weave kit by following old designs from old } \\
\text { traditional pillows. Traditionally, we wove and } \\
\text { produced kit pillows by ourselves for many years. We } \\
\text { normally start weaving kit when we are } 17-18 \text { years } \\
\text { old. }\end{array}$ & $\begin{array}{l}\text { K.capture - village } \\
\text { culture }\end{array}$ \\
\hline 15 & $\begin{array}{l}\text { I started weaving plain cloth as the material of a blouse. } \\
\text { It was white cloth weaving with simple loom with } 2 \\
\text { "Kao". Then, I learnt how to weave simple skirt, ' } \mathrm{Pa} \\
\text { Kloa Ma' (Loincloth), and kit pillow. }\end{array}$ & $\begin{array}{l}\text { K.capture - village } \\
\text { culture }\end{array}$ \\
\hline 16 & $\begin{array}{l}\text { I learnt very fast. It was not hard for me to learn how to } \\
\text { weave because I saw my parents weaving and helped } \\
\text { them to weave. }\end{array}$ & $\begin{array}{l}\text { K.capture - village } \\
\text { culture }\end{array}$ \\
\hline
\end{tabular}


(cont.)

\begin{tabular}{|c|c|c|}
\hline $\begin{array}{l}\text { Text } \\
\text { No. }\end{array}$ & Text & Category \\
\hline 18 & $\begin{array}{l}\text { I can do the other because I helped them doing in the } \\
\text { evening. }\end{array}$ & $\begin{array}{l}\text { K.capture - village } \\
\text { culture }\end{array}$ \\
\hline 19 & $\begin{array}{l}\text { I learnt natural dyes from the staff of the handicraft } \\
\text { centre. They told us sorts of barks that can give colours. } \\
\text { Then, we did experiments by ourselves because the } \\
\text { staff didn't know much about trees in our areas. We } \\
\text { found that each bark can give more than one colour. If } \\
\text { we add alum, it will give one colour. If we add lime, it } \\
\text { will give another colour. }\end{array}$ & $\begin{array}{l}\text { K.capture - village } \\
\text { culture }\end{array}$ \\
\hline 52 & $\begin{array}{l}\text { We have seen our seniors using makleo for fishnet } \\
\text { dyeing. We can collect makleo only once a year in this } \\
\text { season. So, we have planned to collect and ferment } \\
\text { them for using whole year. }\end{array}$ & $\begin{array}{l}\text { K.capture - village } \\
\text { culture }\end{array}$ \\
\hline 38 & $\begin{array}{l}\text { We know what kinds of cloths are good because we } \\
\text { have woven for long time. In addition, the office staff } \\
\text { also tells us our mistake, we have to record that mistake } \\
\text { and talk among the members in the village. }\end{array}$ & K.capture - working \\
\hline 41 & $\begin{array}{l}\text { I had been often for handicraft fairs, such as Thai craft } \\
\text { fair. We need 3-4 people for Thai craft fair. We have to } \\
\text { demonstrate cotton spinning and weaving next month. }\end{array}$ & K.capture - working \\
\hline 53 & $\begin{array}{l}\text { Natural dyeing depends on seasons, such as bark } \\
\text { dyeing is hard to do in rainy season because bark } \\
\text { absorbs a lot of water, but fruit dyeing is good to do in } \\
\text { rainy season. }\end{array}$ & K.capture - working \\
\hline 56 & $\begin{array}{l}\text { We have to hew bark in order to check a colour. It } \\
\text { gives the colour the same as oil wood colour. For } \\
\text { instance, it will give red colour, if its oil wood is red } \\
\text { colour. }\end{array}$ & K.capture - working \\
\hline 63 & $\begin{array}{l}\text { We ask people in Roi-et province to make it, but they } \\
\text { can't do Jug Jig design. They told us that they can do, } \\
\text { but it wasn't the Jug Jig design. So, we have to do it by } \\
\text { ourselves. }\end{array}$ & K.capture - working \\
\hline 64 & $\begin{array}{l}\text { Grandma saw Jug Jig design when she went on a study } \\
\text { tour. She drew the design and prepard the loom by } \\
\text { herself. }\end{array}$ & K.capture - working \\
\hline 51 & $\begin{array}{l}\text { Generally, makleo is used to make black colour for silk. } \\
\text { At the beginning, there was no any village dyeing with } \\
\text { this technique. Many members asked about this } \\
\text { technique. }\end{array}$ & $\begin{array}{l}\text { K.creation - } \\
\text { experiment }\end{array}$ \\
\hline
\end{tabular}




\begin{tabular}{|c|c|c|}
\hline $\begin{array}{l}\text { Text } \\
\text { No. }\end{array}$ & Text & Category \\
\hline 56 & $\begin{array}{l}\text { We have to hew bark in order to check a colour. It } \\
\text { gives the colour the same as oil wood colour. For } \\
\text { instance, it will give red colour, if its oil wood is red } \\
\text { colour. }\end{array}$ & $\begin{array}{l}\text { K.creation - } \\
\text { experiment }\end{array}$ \\
\hline 20 & $\begin{array}{l}\text { We have experiments together among 3-4 members. } \\
\text { Each one brings any bark that is available to use. }\end{array}$ & $\begin{array}{l}\text { K.creation - } \\
\text { experiment }\end{array}$ \\
\hline 47 & $\begin{array}{l}\text { Normally, we use previous designs. We just change } \\
\text { colours. However, we have designed new designs, such } \\
\text { as dinosaur and forest. We have to draw on paper, then } \\
\text { we have to try weaving. If it isn't good, we will take it } \\
\text { out. }\end{array}$ & K.creation - practice \\
\hline 49 & $\begin{array}{l}\text { We did this colour because we have makleo and } \\
\text { Manwan mango at our forest. Mangwan mango can be } \\
\text { found at paddy field. We are also good in brown colour } \\
\text { with "Du" and iron water or lime water. Other villages } \\
\text { have difficulty in finding these trees because they did } \\
\text { not have a small forest at their paddy fields, such as } \\
\text { Nong Thum. }\end{array}$ & K.creation - practice \\
\hline 5 & $\begin{array}{l}\text { I used to weave big blankets with grandma because } \\
\text { they need two people to weave at the same time. }\end{array}$ & K.creation - working \\
\hline 47 & $\begin{array}{l}\text { Normally, we use previous designs. We just change } \\
\text { colours. However, we have designed new designs, such } \\
\text { as dinosaur and forest. We have to draw on paper, then } \\
\text { we have to try weaving. If it isn't good, we will take it } \\
\text { out. }\end{array}$ & $\begin{array}{l}\text { K.creation - } \\
\text { working }\end{array}$ \\
\hline 49 & $\begin{array}{l}\text { We did this colour because we have makleo and } \\
\text { Manwan mango at our forest. Mangwan mango can be } \\
\text { found at paddy field. We are also good in brown colour } \\
\text { with "Du" and iron water or lime water. Other villages } \\
\text { have difficulty in finding these trees because they did } \\
\text { not have a small forest at their paddy fields, such as } \\
\text { Nong Thum. }\end{array}$ & K.creation - working \\
\hline 57 & $\begin{array}{l}\text { Mangwan mango can give red colour, but young } \\
\text { Mangwan mango tree would give light colour, so we } \\
\text { have to add copper sulfate in order to make dark colour. } \\
\text { Red colour from Mangwan mango is different from red } \\
\text { colour of sticklac which gives pink-red. }\end{array}$ & K.creation - working \\
\hline 70 & $\begin{array}{l}\text { We started rug weaving because the office ordered. We } \\
\text { did it with trial and error. At the beginning, it didn't } \\
\text { have patterns on rug, because we used metal reeds. It } \\
\text { would give a pattern when using wooden reed. We } \\
\text { found this technique, because only grandma's rugs had } \\
\text { a pattern. She was only one member that used wooden } \\
\text { reed. }\end{array}$ & K.creation - working \\
\hline
\end{tabular}


(cont.)

\begin{tabular}{|c|c|c|}
\hline $\begin{array}{l}\text { Text } \\
\text { No. }\end{array}$ & Text & Category \\
\hline 41 & $\begin{array}{l}\text { I had been often for handicraft fairs, such as Thai craft } \\
\text { fair. We need 3-4 people for Thai craft fair. We have to } \\
\text { demonstrate cotton spinning and weaving next month }\end{array}$ & $\begin{array}{l}\text { K.sharing - } \\
\text { customers }\end{array}$ \\
\hline 26 & $\begin{array}{l}\text { The participants of the trip trained us when they came } \\
\text { back. }\end{array}$ & K.sharing - forum \\
\hline 27 & $\begin{array}{l}\text { the participants who went to Roi-et trained us how to } \\
\text { make Kit with "Thong Leang" technique that keeps kit } \\
\text { designs on the loom ("Kao") for later weaving. Before } \\
\text { using Thong Leang technique, we had to prepare kit } \\
\text { designs every time of weaving. }\end{array}$ & K.sharing - forum \\
\hline 5 & $\begin{array}{l}\text { I used to weave big blankets with grandma because } \\
\text { they need two people to weave at the same time. }\end{array}$ & K.sharing - working \\
\hline 30 & $\begin{array}{l}\text { Now, we teach only members in our village. They call } \\
\text { us to give advices at their home when they cannot do. }\end{array}$ & K.sharing - working \\
\hline 31 & $\begin{array}{l}\text { We help them design cloth when they do thread } \\
\text { preparing. We take about a day in helping them design } \\
\text { new plain cloth. }\end{array}$ & K.sharing - working \\
\hline 32 & $\begin{array}{l}\text { We help them in any problems, such as wrong patterns } \\
\text { of Kit and binding thread. We don't charge them } \\
\text { because we have to help each other in the group. }\end{array}$ & K.sharing - working \\
\hline 40 & $\begin{array}{l}\text { After that, we have a village meeting to share } \\
\text { information from the committee meeting. For instance, } \\
\text { the committee decided to stop blanket weaving in the } \\
\text { low selling period. We had to tell members in the } \\
\text { village to stop weaving until we had orders. }\end{array}$ & K.sharing - working \\
\hline 59 & $\begin{array}{l}\text { I used to weave it with grandma but now she is busy } \\
\text { with her grandson. So, I’ve to weave it with another } \\
\text { grandma. She used to weave it with her daughter, but } \\
\text { now she has gone to work. }\end{array}$ & K.sharing - working \\
\hline 38 & $\begin{array}{l}\text { We know what kinds of cloths are good because we } \\
\text { have woven for long time. In addition, the office staff } \\
\text { also tells us our mistake, we have to record that mistake } \\
\text { and talk among the members in the village. }\end{array}$ & K.sharing - working \\
\hline 34 & $\begin{array}{l}\text { Nowadays, I cannot do it because my eyesight is not } \\
\text { good. I have to ask some people to prepare kit pattern } \\
\text { ("Keab Kao") for me, then, I can weave Kit. }\end{array}$ & $\begin{array}{l}\text { K.transfer - village } \\
\text { culture }\end{array}$ \\
\hline
\end{tabular}


(cont.)

\begin{tabular}{|c|c|c|}
\hline $\begin{array}{l}\text { Text } \\
\text { No. }\end{array}$ & Text & Category \\
\hline 66 & $\begin{array}{l}\text { I used to prepare loom for them, but now my eyesight } \\
\text { is not good. I haven't prepared kit design as well. I ask } \\
\text { someone to do it. }\end{array}$ & $\begin{array}{l}\text { K.transfer - village } \\
\text { culture }\end{array}$ \\
\hline 48 & $\begin{array}{l}\text { Also, grandma who is my neighbour she made red } \\
\text { colour with 'Mangwan' mango and redyed with } \\
\text { makleo. }\end{array}$ & K.utilising \\
\hline 54 & $\begin{array}{l}\text { Previously, we soaked cloth at the buffalo mire over } \\
\text { night. Now, we don't have a buffalo mire. We have to } \\
\text { take mud from the field to do it at home and can soak } \\
\text { only a few minutes. }\end{array}$ & K.utilising \\
\hline 55 & $\begin{array}{l}\text { My neighbour just did this technique when she made } \\
\text { red colour. Other villages also use this technique. Mud } \\
\text { can help to make darker colour. Sawai just did Samol } \\
\text { dyeing with mud last month. }\end{array}$ & K.utilising \\
\hline 57 & $\begin{array}{l}\text { Mangwan mango can give red colour, but young } \\
\text { Mangwan mango tree would give light colour, so we } \\
\text { have to add copper sulfate in order to make dark colour. } \\
\text { Red colour from Mangwan mango is different from red } \\
\text { colour of sticklac which gives pink-red. }\end{array}$ & K.utilising \\
\hline 68 & $\begin{array}{l}\text { Rug has designs the same as blanket but it is smaller } \\
\text { and needs specific warp preparing in order to make } \\
\text { float pattern. }\end{array}$ & K.utilising \\
\hline 71 & $\begin{array}{l}\text { These village members mainly weave big blankets } \\
\text { because other villages don't want to do. We used to } \\
\text { weave place mats but currently, Ban Nongyanlan's } \\
\text { members do. We have skills in blanket weaving } \\
\text { because we used to weave blankets for our own } \\
\text { consumption but they were simple patterns woven with } \\
\text { "2-Kao". }\end{array}$ & $\begin{array}{l}\text { K.utilising - local } \\
\text { wisdom }\end{array}$ \\
\hline 52 & $\begin{array}{l}\text { We have seen our seniors using makleo for fishnet } \\
\text { dyeing. We can collect makleo only once a year in this } \\
\text { season. So, we have planned to collect and ferment } \\
\text { them for using whole year. }\end{array}$ & $\begin{array}{l}\text { K.utilising - local } \\
\text { wisdom }\end{array}$ \\
\hline 19 & $\begin{array}{l}\text { I learnt natural dyes from the staff of the handicraft } \\
\text { centre. They told us sorts of barks that can give colours. } \\
\text { Then, we did experiments by ourselves because the } \\
\text { staff didn't know much about trees in our areas. We } \\
\text { found that each bark can give more than one colour. If } \\
\text { we add alum, it will give one colour. If we add lime, it } \\
\text { will give another colour. }\end{array}$ & K.verify - experiment \\
\hline
\end{tabular}


(cont.)

\begin{tabular}{|c|c|c|}
\hline $\begin{array}{l}\text { Text } \\
\text { No. }\end{array}$ & Text & Category \\
\hline 48 & $\begin{array}{l}\text { Also, grandma who is my neighbour she made red } \\
\text { colour with 'Mangwan' mango and redyed with } \\
\text { makleo. }\end{array}$ & K.verify - working \\
\hline 55 & $\begin{array}{l}\text { My neighbour just did this technique when she made } \\
\text { red colour. Other villages also use this technique. Mud } \\
\text { can help to make darker colour. Sawai just did Samol } \\
\text { dyeing with mud last month. }\end{array}$ & K.verify - working \\
\hline 23 & $\begin{array}{l}\text { I have been to Chiang Mai and Leoy. At the beginning, } \\
\text { they went to the south of Thailand to study natural } \\
\text { dyes. Chiang Mai trip was organized for committee } \\
\text { members with the objective of looking for alternative } \\
\text { businesses in order to expand our business. }\end{array}$ & $\begin{array}{l}\text { K-capture - study } \\
\text { tour }\end{array}$ \\
\hline 28 & So, study tours gave us additional knowledge. & $\begin{array}{l}\text { K-capture - study } \\
\text { tour }\end{array}$ \\
\hline 64 & $\begin{array}{l}\text { Grandma saw Jug Jig design when she went for study } \\
\text { tour. She drew the design and prepare loom by herself. }\end{array}$ & $\begin{array}{l}\text { K-capture - study } \\
\text { tour }\end{array}$ \\
\hline 22 & $\begin{array}{l}\text { I’ve not often attended training. I went to Ban Fang, } \\
\text { Hua Thong, Nong Thum. They were workshops instead } \\
\text { of training. We did the colours that we were good at. }\end{array}$ & K-capture - training \\
\hline 33 & $\begin{array}{l}\text { I learnt kit weaving from my sister. She taught me } \\
\text { because I had nothing to do at that time. }\end{array}$ & $\begin{array}{l}\text { K-capture - village } \\
\text { culture }\end{array}$ \\
\hline 68 & $\begin{array}{l}\text { Rug has designs the same as blanket but it is smaller } \\
\text { and specific warp preparing in order to make float } \\
\text { pattern. }\end{array}$ & K-creation - practice \\
\hline 46 & $\begin{array}{l}\text { We can design the cloth by ourselves for monthly } \\
\text { weaving. We have to weave as orders when we have } \\
\text { specific orders. We can mix coloured threads in our } \\
\text { cloths as we like, so each member will make different } \\
\text { designs. }\end{array}$ & K-creation - working \\
\hline 20 & $\begin{array}{l}\text { We have experiments together among 3-4 members. } \\
\text { Each one brings any bark that is available to do. }\end{array}$ & KM culture - learning \\
\hline 31 & $\begin{array}{l}\text { We help them design cloth when they do thread } \\
\text { preparing. We take about a day in helping them design } \\
\text { new plain cloth. }\end{array}$ & KM culture - sharing \\
\hline 65 & $\begin{array}{l}\text { We have taught the members in this village to weave } \\
\text { and prepare looms. But they like to ask people in Roi-et } \\
\text { to prepare for them. }\end{array}$ & K-sharing - training \\
\hline 67 & $\begin{array}{l}\text { I used to teach the villagers in prepare Kit design with } \\
\text { "Thong leang" technique, but now I can help them to } \\
\text { fix the problem of Kit weaving. }\end{array}$ & K-sharing - training \\
\hline
\end{tabular}




\begin{tabular}{|c|l|l|}
\hline $\begin{array}{c}\text { (cont.) } \\
\text { Text } \\
\text { No. }\end{array}$ & \multicolumn{1}{|c|}{ Text } & Category \\
\hline 27 & $\begin{array}{l}\text { The participants who went to Roi-et trained us how to } \\
\text { make Kit with “Thong Leang” technique that keeps kit } \\
\text { designs on the loom (“Kao") for later weaving. Before } \\
\text { using Thong Leang technique, we had to prepare kit } \\
\text { designs every time of weaving. }\end{array}$ & K-utilising \\
\hline 36 & $\begin{array}{l}\text { For cloth checking, plain cloth will be checked texture, } \\
\text { then the committee records amount of cloth of each } \\
\text { member. }\end{array}$ & K-utilising \\
\hline
\end{tabular}




\section{Appendix E \\ Basic knowledge of hand weaving}

\section{Traditional Isan hand weaving tools and techniques}

1.1 Traditional Isan hand weaving loom

Traditional Isan hand weaving loom can be viewed as a rectangular block which comprises several parts as illustrated in figure E1. This research will explain only some parts of the loom which have been mentioned in this research in order to expand understanding of readers reading this thesis.

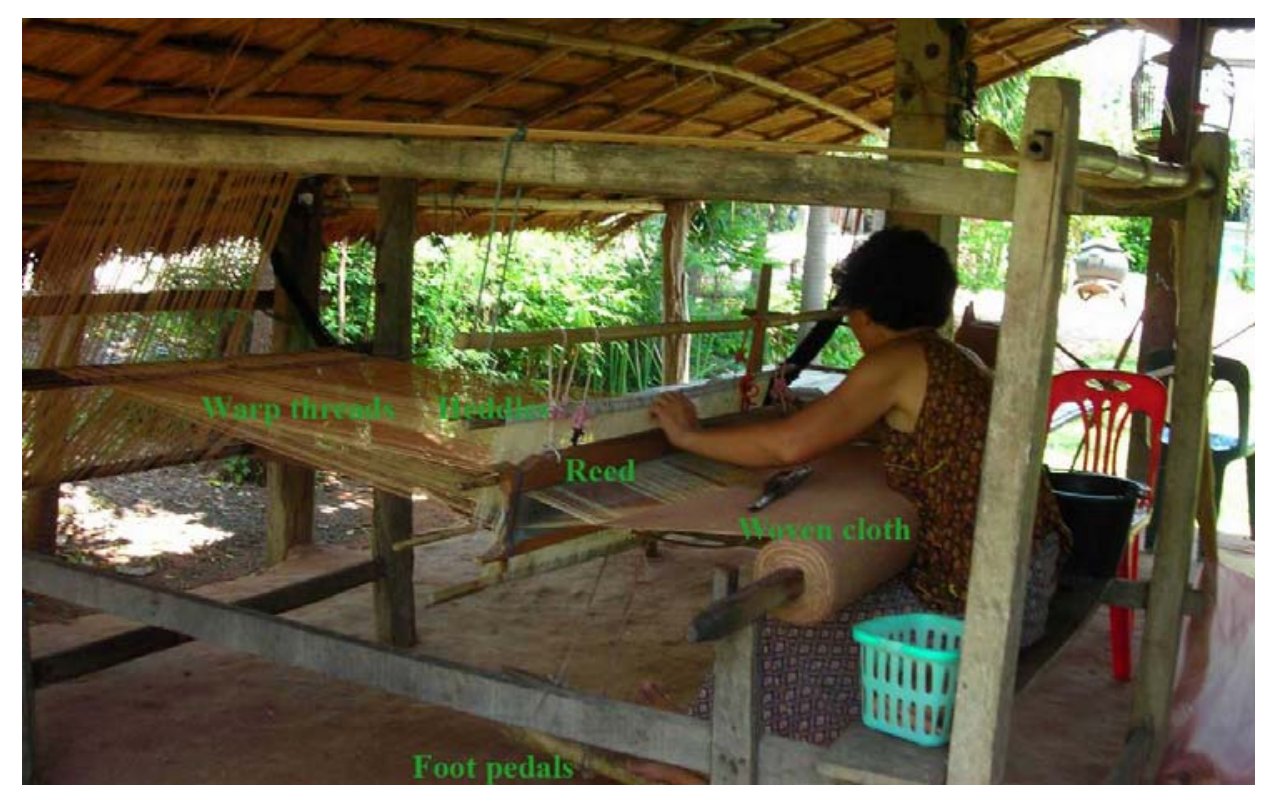

Figure E1: A traditional Isan hand weaving loom

\subsubsection{Reed}

Reed looks like a comb that can be made of wood or metal. Reed has the function of pounding weft threads (Black, 1980).

\subsubsection{Heddle}

A heddle comprises strings which have holds for passing warps. The heddle is located within harness frames tied to the heddle horses or suspended on the top beams of the loom (Black, 1980). The heddle is controlled by a foot pedal. General cloth weaving looms have at least two heddles with two foot pedals. 


\subsubsection{Vertical string-heddle}

A vertical string-heddle is an extra heddle employed to make kit design. Long vertical string-heddle is knows as a "Thong Leang” heddle.

\subsection{Hand weaving techniques}

\subsubsection{3-Kao technique}

3-Kao technique is weaving with three heddle shafts in order to make particular float patterns. Generally, float-weaving can be made of two heddle shafts and up to sixteen shafts (Conway, 1992). 3-Kao weaving is controlled by three foot pedal stepping.

\subsubsection{4-Kao technique}

4-Kao technique is weaving with four heddle shafts in order to make particular float patterns for blankets.

\subsubsection{Kondai}

Kon dai is a process of warp thread preparation, which is a part of loom preparing for weaving. Kon dai comprises the processes of warp pattern design, length calculation and thread need calculation (Black, 1980).

\subsubsection{Thong leang technique}

Thong leang is a technique for making keeping kit designs with bamboo sticks on a vertical string-heddle suspended on a loom. Figure E2 shows a thong leang heddle shaft. 


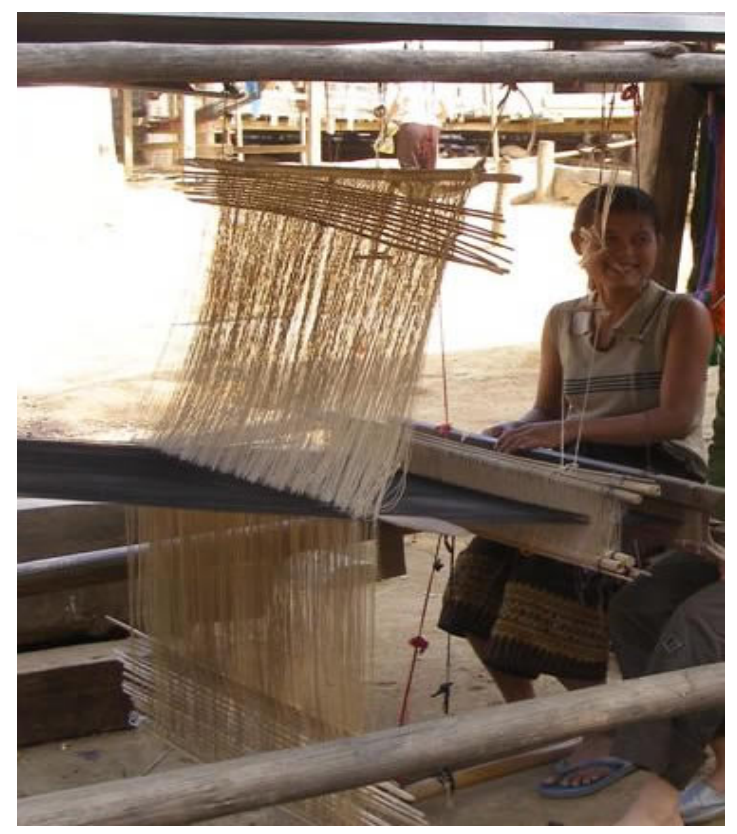

Figure E2 A weaving loom with thong leang heddle shaft as a supplementary part (noyshop.com, 2004).

\section{Traditional Isan cloths}

2.1 Blanket with float-pattern

Blankets are made of cotton with a float-pattern. A respondent in this research explained that there are many traditional float patterns in Isan but "Bug Nuay" (diamond float pattern) is the common float pattern for Isan blankets. Figure E3 illustrates a diamond float pattern. Blankets can also be decorated with stripe pattern or mudmee pattern

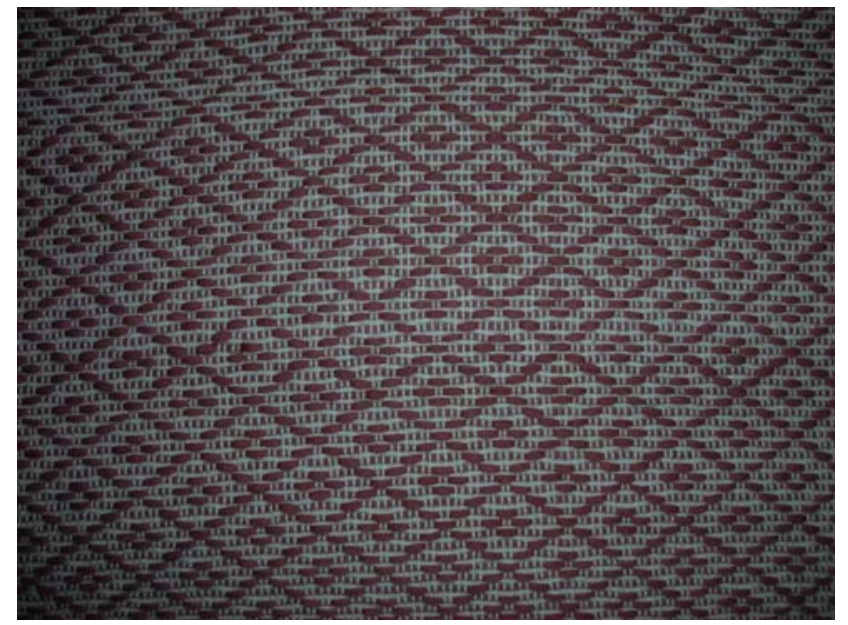

Figure E3 A diamond float pattern of a Prae Pun big blanket 


\subsection{Kit}

Kit is supplementary weft woven cloth (Maxwell, 2003). Kit normally is a pattern used for pillows, mattresses and ceremonial banners. The pattern of kit is made by using bamboo sticks to create patterns of supplementary weft (Conway, 1992). In order to keep the patterns for long-lasting weaving, weavers would store bamboo sticks patterns on a vertical string-heddle harness suspended on the loom (see figure E2). Figure E4 illustrates an example of kit design.

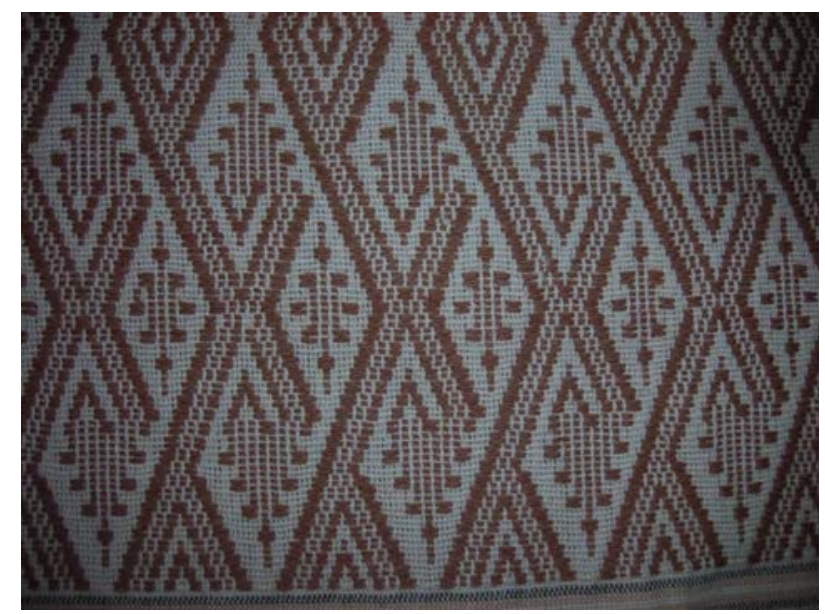

Figure E4 An example of kit design

\subsection{Loincloth}

Loincloth is known as "Pha Kao Ma". It is plaid cloth woven for multi-purpose use. Traditionally, it is men's cloth, used as bathing wraps, waistbands, sweat-cloths and ceremonial shawls (Conway, 1992). However, women also use loincloth as sweatcloths and temporary baby cradles.

\subsection{Mudmee}

Mudmee is supplementary weft woven cloth whose patterns are prepared by a resist dyeing process of the weft (Maxwell, 2003). Traditionally, mudmee is plain woven cloth which is referred to as a tube skirt known as "Phasin" and made of silk. Currently, mudmee is applied to raw materials to make modern dresses and products. Figure E5 shows an example of mudmee design. 


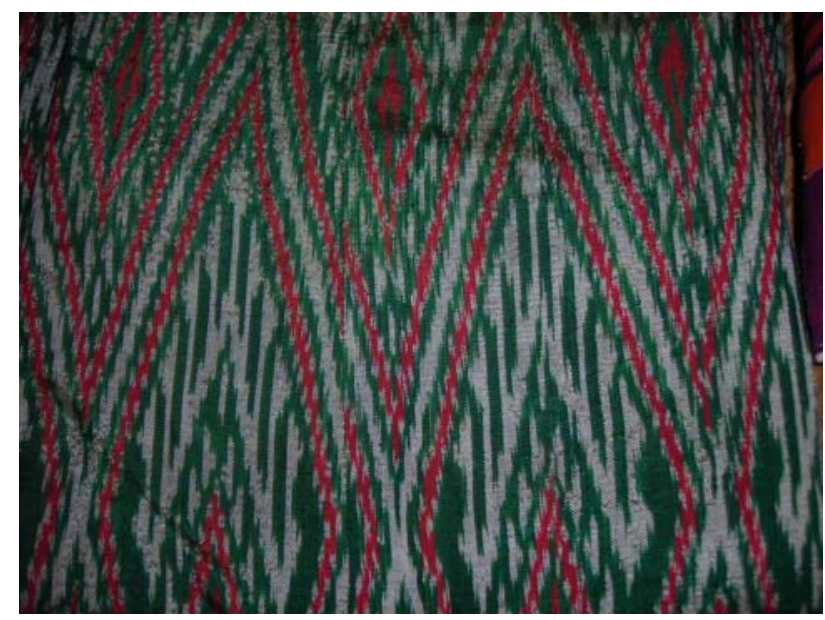

Figure E5 An example of mudmee design

\subsection{Sarong}

Sarong is a plaid tube skirt for men worn as casual cloth. Traditionally, it is made of silk (Conway, 1992).

\subsection{Tung}

Tung is a ceremonial banner with supplementary-weft patterns, especially kit pattern. In Isan, a well-know ceremonial banner is the "Bun Phawes" banner. Figure E6 shows an example of a Bun Phawes banner. Bun Phawes is a major Isan merit ceremony which takes place after rice harvesting (Conway, 1992).

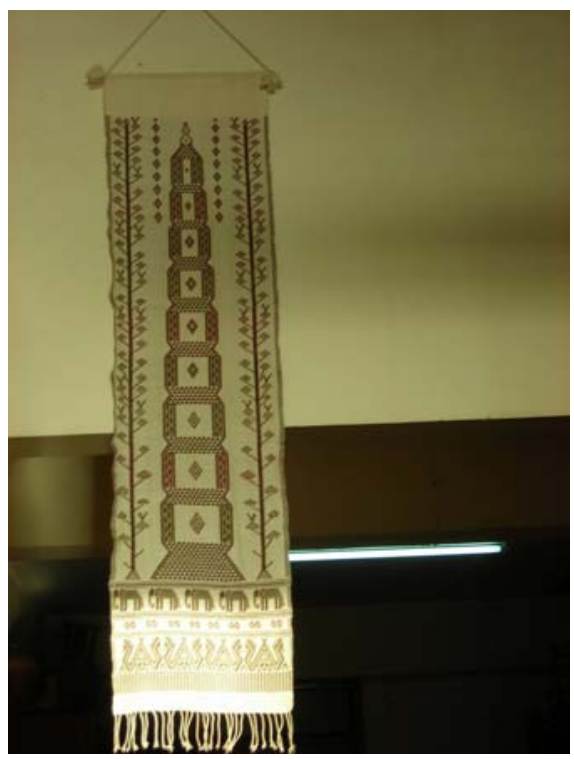

Figure E6 An example of a Bun Phawes banner 
2.7 Saifon cloth

Saifon is plain woven cloth which can be considered as supplementary weft cloth. It is woven with Mudmee weft. Prae Pun's members accidentally discovered it while they were using Mudmee thread as weft and weaving without considering the proper design. 